Biblioteca di Storia

-32 - 

Roberto Bianchi

\section{Una storia, un archivio}

Idalberto Targioni nell'Italia tra Ottocento e Novecento 
Una storia, un archivio : idalberto Targioni nell'Italia tra Ottocento e Novecento / Roberto Bianchi. - Firenze : Firenze University Press, 2018.

(Biblioteca di Storia ; 32)

http://digital.casalini.it/9788864537733

ISBN 978-88-6453-772-6 (print)

ISBN 978-88-6453-773-3 (online PDF)

ISBN 978-88-6453-774-0 (online EPUB)

Progetto grafico di Alberto Pizarro Fernández, Pagina Maestra snc Immagine di copertina: Idalberto Targioni e famiglia, Archivio Idalberto Targioni

\section{Certificazione scientifica delle Opere}

Tutti i volumi pubblicati sono soggetti ad un processo di referaggio esterno di cui sono responsabili il Consiglio editoriale della FUP e i Consigli scientifici delle singole collane. Le opere pubblicate nel catalogo della FUP sono valutate e approvate dal Consiglio editoriale della casa editrice. Per una descrizione più analitica del processo di referaggio si rimanda ai documenti ufficiali pubblicati sul catalogo on-line della casa editrice (www.fupress.com).

Consiglio editoriale Firenze University Press

A. Dolf (Presidente), M. Boddi, A. Bucelli, R. Casalbuoni, M. Garzaniti, M.C. Grisolia, P. Guarnieri, R. Lanfredini, A. Lenzi, P. Lo Nostro, G. Mari, A. Mariani, P.M. Mariano, S. Marinai, R. Minuti, P. Nanni, G. Nigro, A. Perulli, M.C. Torricelli.

Lapresenteoperaè rilasciata neiterminidella licenza Creative Commons Attribution 4.0 International (CC BY 4.0: http://creativecommons.org/licenses/by/4.0/legalcode).

This book is printed on acid-free paper

CC 2018 Firenze University Press

Università degli Studi di Firenze

Firenze University Press

via Cittadella, 7, 50144 Firenze, Italy

www.fupress.com

Printed in Italy 


\section{Sommario}

Introduzione VII

Abbreviazioni e sigle XIII

Idalberto Targioni, una biografia $\quad 1$

L'Archivio Idalberto Targioni 41

a cura di Daniele Lovito

APPENDICE

Targioni in privato. Una testimonianza 149

di Simonetta Chiappini

Indice dei nomi 159 



\section{Introduzione}

Una povera donna montanina lieta recava al petto un trovatello preso là nel buglione, ove s'insacca dal matrimonio e dallo stupro a gara, o legittima o no, l'umana carne.

Oh benedetta, miseri innocenti, la pubblica pietà che vi ricovra nudi, piangenti, abbandonati! A voi

il casto grembo della cara madre e del tetto paterno il santo asilo che dà l'essere intero, e dolcemente

l'animo leva a dignità di vita, error, vergogna, delitto e miseria chiude per sempre! Crescerete soli, soli all'affetto e malsecuri in terra; al disamor di genitori ignoti, come la pianta che non ha radice, maledicendo! (Giuseppe Giusti, Gita da Firenze a Montecatini, 18 ottobre $1846^{1}$ )

Nato nel 1868 e morto nel 1930, Idalberto Targioni fu trovatello, contadino, operaio, poeta autodidatta, popolare cantore di rime in ottava, scrittore e studioso, attivista sindacale e antimilitarista, socialista e sindaco di Lamporecchio (un Comune in Provincia di Pistoia, il cui circondario all'epoca apparteneva a quella di Firenze), interventista e fascista della prima ora, uomo del regime infine caduto in ombra.

Fu una figura controversa e discussa, sempre al centro di polemiche e coinvolta nel turbine dei conflitti politici e sociali che segnarono il passaggio dall'epoca ben rappresentata nel Quarto stato di Pellizza da Volpedo al nuovo mondo del Novecento con i suoi estremi, aperto dalla Prima guerra mondiale e dalla crisi che portò il fascismo al potere. Fu un protagonista del proprio tempo sempre in contatto, in corrispondenza e talvolta in contrasto

1 Citazione inserita da Idalberto Targioni come incipit in I miei ricordi d'infanzia, ovvero I "proverbi" di mamma Giuditta, Polli-Targioni, Firenze 1912, e con qualche piccola modifica in Archivio Idalberto Targioni (AIT), Scatola 3.31, Cinquant'anni della mia vita (Ricordi e memorie) 1920, p. 9: questa autobiografia dattiloscritta è composta da circa 200 pagine non numerate (nn.), salvo le prime 53, e contiene molte inserzioni. 
con altri attori contemporanei più noti, come il Gran maestro del Grande Oriente d'Italia Domizio Torrigiani o come, per altre vie e con esiti diversi, Benito Mussolini.

Sarebbe banale definire la sua vita come un paradosso; sta di fatto che Targioni fu un risparmiatore parsimonioso e un giocatore d'azzardo; marito e padre attento alle vicende familiari, nonché appassionato tessitore di altre relazioni sentimentali. Fieramente noto per il suo anticlericalismo, si riallineò con la Chiesa alla viglia dei Patti Lateranensi e mostrò un intimo avvicinamento alla religiosità nell'ultimo scorcio di vita. Combattente convinto della necessità di lottare per la giustizia sociale, i suoi ideali ebbero sempre una coloratura malinconica e un fondo di pessimismo permanente. Insomma, Idalberto Targioni fu al contempo una figura straordinaria, singolare e poliedrica, e un uomo che incarnò un percorso di vita non atipico per l'Italia tra Ottocento e Novecento.

La sua non è una vicenda sconosciuta. Però, va detto che se abbiamo a disposizione una solida produzione storiografica sulla storia della Toscana dall'Unità al fascismo ${ }^{2}$, sulla storia dei partiti e del passaggio dal socialismo al mussolinismo di molti intellettuali, dirigenti politici e sindacali ${ }^{3}$, come pure sulle conseguenze degli irriducibili scontri tra interventisti e neutralisti che dal 1914 segnarono per più di un lustro la storia italiana ${ }^{4}$, manca tuttora una biografia di Targioni.

Le ragioni di questa lacuna non vanno cercate scomodando ipotesi di complotto ideologico da parte degli storici 'di sinistra' ai danni di un uomo che passò 'dal rosso al nero', che trascorse gran parte della vita in Toscana (ovvero in una regione che in età repubblicana sarebbe stata vista come rossa, irrobustita da un solido associazionismo e da una fin troppo celebrata tradizione

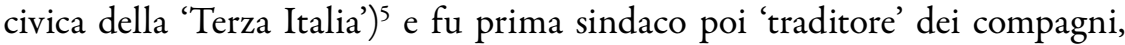
squadrista e collaboratore del regime fascista in un paese collocato all'inter-

2 Ovviamente, non è possibile richiamare le principali opere sul tema; per orientarsi cfr. G. Mori (a cura di), Storia d'Italia. Le regioni dall'Unità a oggi. La Toscana, Einaudi, Torino 1986.

${ }^{3}$ La centralità assunta dalla biografia di Mussolini ha un po' inevitabilmente messo in ombra i percorsi dei tanti uomini e donne che con la guerra furono sedotti dalle «nefaste meraviglie» del nuovo secolo: A. Gibelli, L'officina della guerra. La Grande guerra e le trasformazioni del mondo mentale, Bollati Boringhieri, Torino 1991, p. 27.

${ }^{4}$ Per un'introduzione cfr. i saggi di G. Procacci, G. Sabbatucci e A. Lyttelton in G. Sabbatucci, V. Vidotto (a cura di), Storia d'Italia, vol. 4: Guerre e fascismo 1914-1943, Laterza, Roma-Bari 1997, pp. 3-243.

5 Sulla 'tradizione civica' il richiamo è a R.D. Putnam, La tradizione civica nelle regioni italiane, Mondadori, Milano 1993, e sulla Terza Italia ad A. Bagnasco, Tre Italie. La problematica territoriale dello sviluppo italiano, il Mulino, Bologna 1977, e ai dibattiti suscitati da queste opere. Sulla permanenza del mito della 'Toscana rossa' cfr., ad esempio, M. Lancisi, Berlusconi ordinò «Detoscanizzare l'Italia», ma la regione rossa ha resistito alla sfida, «Il Tirreno», 13 novembre 2011; S. Poli, L'uomo che voleva detoscanizzare l'Italia, «la Repubblica», 23 gennaio 2010; sul neologismo 'detoscanizzare' cfr. il dizionario Treccani: <https://goo. 
no di quella «grande $\mathrm{Y}$ rovesciata» che nella seconda metà del XX secolo avrebbe teso a riprodurre sul piano elettorale l'area in cui il Partito comunista italiano negli anni Settanta raccoglieva tra il 50 e il $70 \%$ dei voti ${ }^{6}$.

Fu anzi Libertario Guerrini - già partigiano, comunista e poi anche storico - a fornire negli anni Cinquanta una prima ricostruzione del ruolo di questo «poeta estemporaneo» per la «storia del movimento contadino empolese ${ }^{7}$. Una ventina di anni dopo toccò ad Aldo Morelli - anch'egli uomo di sinistra e storico, in seguito presidente della Provincia di Pistoia e poi sindaco di centrosinistra a Lamporecchio - il compito di scrivere la voce su Targioni per il più importante dizionario biografico del movimento operaio italiano ${ }^{8}$. In tempi più recenti, Claudia Poli avrebbe valorizzato il ruolo di Targioni a Lamporecchio e presentato un intervento sul «fenomeno Targioni» nell'ambito di un seminario sul fascismo in Toscana'. Adesso è possibile trovare sulla piattaforma Youtube anche la videolettura di un testo di Targioni ed è in corso di pubblicazione la voce dedicata nel Dizionario Biografico degli Italiani ${ }^{10}$.

$\mathrm{gl} / 7 \mathrm{xmUjY}>$. I siti Internet sono stati controllati nell'ultima decade di aprile 2018; per le abbreviazioni è stato usato lo strumento goo.gl.

${ }^{6} \mathrm{La}$ «grande Y rovesciata» «si staglia nel centro della regione, dalla punta settentrionale di Barberino di Mugello a Piombino e Sarteano, rispettivamente a sud-ovest e a sud-est»: F. Andreucci, A. Pescarolo, La formazione delle regioni "rosse" in Italia: il caso della Toscana, in Gli spazi del potere. Aree, regioni, Stati: le coordinate territoriali della storia contemporanea, a cura di Id., La Casa Usher, Firenze 1989, p. 130. Cfr. R. Bianchi, Il centro in periferia. Società e politica nella Valdelsa contemporanea (1900-1980), in P. Ginsborg, F. Ramella (a cura di), Un'Italia minore. Famiglia, istruzione e tradizioni civiche in Valdelsa, Giunti, Firenze 1999, pp. 32-108; G. Becattini, Scritti sulla Toscana, 4 voll., Le Monnier, Firenze 2007.

L. Guerrini, Un poeta estemporaneo, Idalberto Targioni, nella storia del movimento contadino dell'empolese, «Movimento operaio», VII (3-4), 1955, pp. 511-530; cfr. Id., Il movimento operaio nell'Empolese 1861-1946, Editori Riuniti, Roma 1970, pp. 114-115. Nel 1944 Guerrini aveva militato nelle Squadre di azione patriottica della zona di Empoli, cfr. <https://goo. $\mathrm{gl} / \mathrm{VmwAH} 1>$. Una selezione del testo di Guerrini venne ripubblicata nel fascicolo 7 inserito nella cartella I sindacalisti Pietro Bellotti, Paola Tarelli, Gino Castagno, Franco Mariani, Salvatore Carnevale, Bruno Di Pol e Idalberto Targioni, a cura di C. Strada e S. Molinari, Associazione Amici dell'Avanti!, Milano 1983.

${ }^{8}$ Cfr. A. Morelli, Targioni Idalberto, in F. Andreucci, T. Detti (a cura di), Il movimento operaio italiano. Dizionario biografico 1853-1943, Editori Riuniti, Roma 1978, vol. V, pp. 6-13; A. Morelli e L. Tomassini, Socialismo e classe operaia a Pistoia durante la prima guerra mondiale, Feltrinelli, Milano 1976.

9 Cfr. C. Poli, La vicenda del socialista Targioni e la nascita del fascismo a Lamporecchio, «Quaderni di Farestoria», XIII (2-3), 2011, pp. 133-141; Ead., Lamporecchio nel primo Novecento. Quotidianità e istituzioni, Pistoia, CRT, 2004. Cfr. anche la nota di L. Martini, In memoria di Idalberto Targioni nomo prima tanto amato, poi tanto vituperato e, infine, troppo presto dimenticato, «Il Segno di Empoli», VI (21), 1993, pp. 8-9.

${ }_{10}$ Cfr. <https://goo.gl/DDQ4J1>, dove è letto il testo I miei Ricordi d' infanzia, cit.; R. Bianchi, Targioni, Idalberto, in Dizionario Biografico degli Italiani, Istituto dell'Enciclopedia italiana Treccani, Roma, in corso di pubblicazione. 
Inoltre, in modo più o meno occasionale, la sua vita e le sue opere sono state oggetto di studi che riguardano la storia delle identità italiane ${ }^{11}$, della società locale, del movimento socialista e del fascismo ${ }^{12}$, della massoneria ${ }^{13}$, della cultura popolare ${ }^{14}$, socialista e sovversiva ${ }^{15}$. Ma la mancanza di una vera e propria biografia va capita considerando lo stato più generale degli studi sulla Toscana tra Otto e Novecento e sui suoi protagonisti, e quindi le tante ricerche ancora da fare su figure e soggetti importanti ma non di primissimo piano; mentre, in realtà, sappiamo che proprio i percorsi dei quadri intermedi dei partiti, il 'ceto medio' degli intellettuali, dei corpi istituzionali e delle reti associative possono dirci molto sui movimenti profondi della società contemporanea ${ }^{16}$.

11 In A. Nesti, Provincialia. Scavi sull'identità degli italiani, Polistampa, Firenze 1999, cfr. il capitolo su Mito e storia a Lamporecchio, pp. 39 sgg.

${ }_{12}$ Cfr. R. Risaliti, Il movimento socialista a Pistoia durante la Prima guerra mondiale, Comune di Pistoia, Pistoia 1970; Id., La lotta sindacale e politica a Pistoia dal dicembre 1918 al luglio 1920, Comune di Pistoia, Pistoia 1970; Id., Nascita e affermazione del fascismo a Pistoia, «Farestoria», III (1), 1983; M. Francini, Primo dopoguerra e origini del fascismo a Pistoia, Feltrinelli, Milano 1976; F. Snowden, The Fascist Revolution in Tuscany 1919-1922, Cambridge University Press, Cambridge 1990; G. Petracchi, La genesi del fascismo a Pistoia, in 28 ottobre e dintorni. Le basi sociali e politiche del fascismo in Toscana, Polistampa, Firenze 1994, pp. 97-121; Id., Storia di Pistoia, vol. IV: Nell'età delle rivoluzioni 1777-1940, a cura di Id., Le Monnier, Firenze 2000, pp. 390-400; S. Bartolini, Una passione violenta. Storia dello squadrismo fascista a Pistoia (1919-1923), Comune di Pistoia, Pistoia 2011; Id., La mezzadria nel Novecento. Storia del movimento mezzadrile tra lavoro e organizzazione, Settegiorni, Pistoia 2015; R. Bianchi, Grande guerra, Grande dopoguerra. Lotte politiche e conflitti sociali a Pistoia (1914-1921), in Pistoia nell'Italia unita. Identità cittadina e coscienza nazionale, Società pistoiese di Storia patria, Pistoia 2012, pp. 263-290.

13 Cfr. M. Francini e G.P. Balli, Il "gran maestro" Domizio Torrigiani (1876-1932), CRT, Pistoia 2003, pp. 19-26; S. Moroni, Massoneria e vita politica a Pistoia e nella Val di Nievole tra guerra e fascismo, in F. Conti (a cura di), Massoneria e società civile. Pistoia e la Val di Nievole dall'Unità al secondo dopoguerra, FrancoAngeli, Milano 2003, pp. 97-129; R. Bianchi, La massoneria e le origini del fascismo in Toscana, «Contemporanea», XVI (4), 2013, pp. 499-522; Id., Massoneria e fascismo in Toscana, in F. Conti (a cura di), La massoneria italiana da Giolitti a Mussolini. Il gran maestro Domizio Torrigiani, Viella, Roma 2014, pp. 109-138.

${ }_{14}$ Cfr. ad esempio N. Baronti (a cura di), Befanate e scherzi in poesia, Raccolta di poesie, prose poetiche e stornelli della tradizione orale di Vinci e del Montalbano, Polistampa, Firenze 2011. Per una testimonianza cfr. M. Brotini, Nuova linfa al manifesto di Marx. In ottava rima, «Left», 26 dicembre 2017, <https://goo.gl/qCtQ9Z>.

${ }^{15}$ Cfr. E. Franzina, Dall'Arcadia in America. Attività letteraria ed emigrazione transoceanica in Italia (1850-1940), Fondazione Giovanni Agnelli, Torino 1996, pp. 153-159; F. Bertolucci, Antonio Gamberi, ateo convinto e fermo socialista. Appunti per una biografia, in A. Gamberi, Poesie per un "liberato mondo". Antologia, a cura di F. Bertolucci e D. Ronco, BFS, Pisa 2004, pp. 11-56. Più in generale, va visto G. Turi, Intellettuali e propaganda nel movimento socialista, in Fare gli italiani. Scuola e cultura nell'Italia contemporanea, a cura di Id. e Simonetta Soldani, vol. 1: La nascita dello Stato nazionale, il Mulino, Bologna 1993, pp. 459-501.

${ }^{16}$ Per una storia sociale del fascismo cfr. P. Dogliani, Il fascismo degli italiani. Una storia sociale, Utet, Torino 2014. 
Oggi, a 150 anni di distanza dalla nascita di Targioni e a un secolo da quella dei Fasci di Combattimento, è possibile e auspicabile avviare una analisi puntuale della sua vita e del suo ruolo grazie alla generosa messa a disposizione dell'Archivio privato - che include la biblioteca personale - da parte dei suoi eredi, in particolare della prof.ssa Simonetta Chiappini, e alla compilazione dell'Elenco di consistenza del fondo a cura del dott. Daniele Lovito, qui pubblicato assieme a questo profilo biografico che potrà essere arricchito dallo studio delle carte ancora da esplorare valorizzando, in particolare, le notizie sulle sue vicende più intime, personali e familiari che sicuramente ebbero legami non casuali con le scelte politiche pubbliche.

La lettura dell'Elenco di consistenza, lo spoglio delle carte conservate nella casa degli eredi a Lamporecchio - finora mai esaminate in modo sistematico $\mathrm{e}$ in attesa di una collocazione archivistica adeguata ${ }^{17}-$, assieme alla consultazione di documenti presso l'Archivio dell'Ospedale degli Innocenti a Firenze, l'Archivio di Stato a Pistoia, quello Comunale a Lamporecchio e quello di Domizio Torrigiani conservato dall'Istituto Storico Toscano della Resistenza e dell'Età contemporanea, oltre alla rilettura delle fonti già note, delle opere, dei fogli volanti, degli articoli scritti da Targioni e, ovviamente, degli studi a disposizione, permetteranno di sviluppare nuove ricerche che, al momento, stiamo impostando.

Si ha l'impressione che attraverso la biografia di Targioni sarà possibile capire qualcosa di più della storia d'Italia tra età liberale e fascismo. Si potranno fornire nuove notizie e spunti di riflessione sulla vita dei bambini abbandonati che a cavallo tra i due secoli passarono - come Targioni - per l'Istituto degli Innocenti, il più antico brefotrofio d'Europa ${ }^{18}$; sulla cultura contadina e i percorsi di formazione e autoformazione nelle campagne e nell'Appennino toscano; sulla storia della lingua, del canto, della poesia e della letteratura popolare; sull'affermazione del socialismo nel mondo rurale; sulle conversioni alla guerra che dal 1914 segnarono le élite d'Italia, come pure ampie porzioni dei ceti medi e settori delle classi popolari; sulle origini del fascismo e la violenza squadrista; sulle dinamiche interne al regime e le peculiarità delle sue articolazioni locali; sul rapporto tra storia e memoria nel Novecento, attraverso

17 Attualmente l'archivio e la biblioteca Targioni si trovano nell'abitazione degli eredi a Lamporecchio, piazza IV novembre 16. Sappiamo che le carte sono state occasionalmente consultate, cfr. ad esempio Martini, In memoria, cit., e Poli, Lamporecchio, cit.

${ }^{18}$ Cfr. S. Filipponi, E. Mazzocchi, L. Sandri (a cura di), Figli d'Italia (1861-1911). Gli innocenti e la nascita di un progetto nazionale per l'infanzia, Alinari 24 ore, Firenze 2011; P. Guarnieri, Dall'accoglienza alla cura: la riforma sanitaria nel brefotrofio degl'Innocenti di Firenze 1890-1918, «Medicina \& storia», 7, 2004, pp. 57-100; G. Di Bello, Senza nome né famiglia. I bambini abbandonati nell'Ottocento, Manzuoli, Firenze 1989; Ead., L'identità inventata. Cognomi e nomi dei bambini abbandonati a Firenze nell'800, Centro Editoriale Toscano, Firenze 1993. 
lo studio delle particolari caratteristiche di un caso locale che per certi aspetti sembra esemplare.

In questa fase della ricerca è quindi possibile fornire una serie di notizie, qualche spunto di riflessione e impostare la ricostruzione della biografia seguendo alcune scansioni di questa storia di vita, dando rilievo a sei fasi sintetizzate dai soprannomi che accompagnarono Targioni durante l'infanzia («il Bastardo»), l'adolescenza («l'Usignolo»), la prima età adulta («il Diavolo rosso»), la mezza età («il Convertito») e l'inizio di quel declino («il Traditore») che lo portò all'oblio e a una vecchiaia senza soprannomi («il Pentito»).

\section{Ringraziamenti}

Sono molte le persone che hanno contribuito alla realizzazione di quest'opera, a cominciare da Simonetta Chiappini, la bisnipote di Idalberto Targioni, che con intelligenza e determinazione ha accompagnato la ricerca, e Daniele Lovito che ha condotto un prezioso e paziente lavoro tra le carte dell'archivio, curando la realizzazione dell'elenco di consistenza. Monica Cetraro, assessore alla cultura del Comune di Lamporecchio, l'Istituto Storico Toscano della Resistenza e dell'Età contemporanea e il suo direttore Matteo Mazzoni, l'Istituto Storico della Resistenza e dell'Età contemporanea di Pistoia, e in particolare il direttore Matteo Grasso, hanno promosso il progetto. Ringrazio il Dipartimento Sagas dell'Università di Firenze che ha sostenuto la pubblicazione del volume e Fulvio Guatelli, direttore della Firenze University Press; l'Archivio dell'Ospedale degli Innocenti di Firenze, in particolare Cinzia Merlino e Lucia Ricciardi che mi hanno aiutato nella ricerca di informazioni e hanno permesso la pubblicazione di immagini dei documenti d'archivio; Roberta Niccoli e Daniele Bartalucci hanno generosamente offerto la foto della targa affissa sulla loro abitazione a San Baronto. Infine, ringrazio chi ha letto, corretto e commentato parti del volume in momenti diversi: Marta Bonsanti, Irene Dati, Elena Gonnelli, Justine Grou-Radenez, Matteo Mazzoni, Simone Neri Serneri, Monica Pacini, Vanessa Roghi, Francesca Tacchi, e soprattutto Simonetta Soldani che mi ha anche messo in contatto con gli eredi Targioni. Il libro è dedicato alla memoria di mio padre, contadino ed emigrante dell'Appennino pistoiese, cresciuto fra i saggi ignoranti di montagna che sapevano Dante a memoria e improvvisavano di poesia. 


\section{Abbreviazioni e sigle}

ACS = Archivio Centrale dello Stato

ADT $=$ Archivio Domizio Torrigiani

AIT = Archivio Idalberto Targioni

AOIF = Archivio dell'Ospedale degli Innocenti di Firenze

ASCL $=$ Archivio Storico del Comune di Lamporecchio

ASP $=$ Archivio di Stato di Pistoia

$\mathrm{CPC}=$ Casellario politico centrale

DGPS $=$ Direzione generale della Pubblica sicurezza

ISRT = Istituto storico toscano della resistenza e dell'età contemporanea

IT = Idalberto Targioni

MI = Ministero degli Interni

O.d.g. $=$ ordine del giorno

PNF = Partito nazionale fascista 



\section{Idalberto Targioni, una biografia}

\section{Il Bastardo: l'infanzia}

Sappiamo che Idalberto Targioni, figlio di ignoti, nacque a Firenze il 19 ottobre 1868, negli anni di Firenze capitale. Dalle carte conservate presso l'Ospedale degli Innocenti (Fig. 1) risulta che quel giorno la levatrice Irene Gragnani, residente in via San Zanobi 116, consegnò il neonato all' istituto di piazza Santissima Annunziata assieme a un «filandente ricamato in lana color verde e solferino rivoltato nel suo attestato» ${ }^{1}$. Come si usava fare, il piccolo segnale di riconoscimento era tagliato a metà; il ricamo, di pochi centimetri quadri, è ancora conservato in una scatolina con molti altri 'segnali' (Fig. 2) 2 . Sull'attestato firmato dalla levatrice si legge: «A di 19 ottobre 1868 io sotto scritta come levatrice mando un caso di bambino con segno di ricamo in lana e nastro scuro ore 8 di sera $»^{3}$, e nel registro dell'Istituto venne annotato che il bambino, il $1814^{\circ}$ neonato affidato all'Ospedale dall' inizio dell'anno, «si fece battezzare col nome di Edelberto, recapito in filza 135, n. 1717». Dopo pochi giorni, il 22 ottobre, Edelberto Targioni venne affidato «a balia a latte alla Luisa moglie di Domenico Rai, del Popolo di S. Basilio a Prunetta ${ }^{4}$.

Questo dato non si ritrova nel racconto autobiografico che nel 1920, a 52 anni, Targioni avrebbe stilato con una macchina per scrivere. Infatti, nell'autobiografia inedita, Targioni non fa menzione di Luisa e Domenico Rai, ma

1 Archivio Ospedale degli Innocenti di Firenze (AOIF), Balie e Bambini 1868, F8a, p. 4a, 1.10.1868-31.12.1868, n. inv. 786, n. d'ordine 1814.

2 AOIF, Segnale di riconoscimento 1868, ottobre II parte, M 1814.

3 AOIF, Recapiti di creature introdotte nel 1868, Filza 135, 1.1.1868-31.12.1868, n. inv. 1429, sotto n. 1757. Sulle levatrici cfr. A. Gissi, Le segrete manovre delle donne. Levatrici in Italia dall'Unità al fascismo, Biblink, Roma 2006.

4 AOIF, Balie e Bambini 1868, cit. 
riferisce di essere stato affidato a Mazzina e Giovanni Gavazzi: una coppia di contadini residenti a Casa Marconi (o Casa Marcone; «Casa di Marconi», si legge nelle memorie), un aggregato di case dell'Appennino pistoiese sulla strada tra Le Piastre e Prunetta, distante meno di un $\mathrm{km}$ in linea d'aria dalla chiesa di San Basilio, a Prunetta ${ }^{5}$. Gli eredi di Targioni confermano questa notizia e Simonetta Chiappini ricorda di aver persino incontrato gli eredi di Giovanni Gavazzi presso la loro abitazione ${ }^{6}$. È possibile che alcuni passaggi del bambino da una famiglia all'altra - con meccanismi di 'subappalto' nella gestione delle risorse fornite dagl'Innocenti agli affidatari, all' insaputa dell' istituto che contrastava queste transazioni -, non siano stati registrati nelle carte dell'archivio dell'ospedale.

Come per tante e tanti altri nocentini (i bambini e le bambine passati dall' istituto), la ricerca delle proprie origini fu un'ossessione che accompagnò Targioni per tutta la vita. Nelle memorie narra di essersi recato all'Istituto degli Innocenti a 32 anni, quindi allo schiudersi del '900, per cercare notizie; ma sicuramente ci andò più volte, visto che nel registro dell'istituto, proprio nella pagina che lo riguarda, si trova inserito il biglietto da visita di «Idalberto Targioni. Consigliere Provinciale e Segretario della Corporazione dell'Agricoltura di Firenze», un cartoncino che - come vedremo - avrebbe potuto essere stampato solo dopo il 1924 .

Targioni ci dice anche che nel 1902 riuscì a ritrovare il primo affidatario, Giovanni Gavazzi; il vecchio, oramai ultraottantenne, in quell'occasione avrebbe raccontato al figlio adottivo che la sua nascita sarebbe stata il frutto dell'«unione clandestina di un principe con una governante della Casa Reale». Era una bella favola difficilmente verificabile e mai confermata, che Targioni avrebbe però reso pubblica nello Schizzo autobiografico inserito nell'introduzione al suo ultimo Canzoniere di poesie sociali, quello del 1912, e che sarebbe stata presa per buona da alcuni studiosi ${ }^{8}$.

Lo Schizzo autobiografico è particolarmente interessante, sia perché mostra l'immagine pubblica di sé che intendeva fornire Targioni a 44 anni, sia perché è soprattutto su quel testo che si sarebbero poi basate le informazioni biografiche pubblicate in sede storica. Il Canzoniere del 1912 uscì negli anni di maggior popolarità e maturità del poeta contadino, prima dell'assunzione di più importanti cariche politiche e istituzionali. Introducendo l'opera antologica, l'autore cercava di catturare la benevolenza dei lettori affermando che «per un semianalfabeta

\footnotetext{
5 AIT, Scatola 3.31, Cinquant'anni, cit., pp. 9 sgg.; cfr. ivi, Scatola 2.19.4, Casa di Marcone, carta ms., s.l., s.d., 1 c. Poesia composta da tre quartine e due terzine.

6 Colloqui con l'autore, 23 e 27 aprile, 12 maggio 2018.

7 Cfr. AIT, Scatola 3.31, Cinquant'anni, cit., pp. $15 \mathrm{sgg}$.

8 I. Targioni, Canzoniere di poesie sociali, Tip. U. Polli, Firenze 1912, p. IX. Questo tipo di racconto delle origini ricorre in situazioni simili; oggi la questione è regolamentata dalla legge 28 marzo 2001, n. 149, cfr. «Gazzetta Ufficiale», 26 aprile 2001.
} 
quale il sottoscritto, che non ha nemmeno fatto le classi elementari, spingere la propria audacia fino al punto di pubblicare un libro, può essere, e giustamente, giudicato atto temerario da rasentar la pazzia ». Poi narrava la propria vita:

Venni al mondo 44 anni fa ai 19 d'ottobre. Il giorno successivo, da mano ignota, fui portato al Brefotrofio (Regio Ospedale degl'Innocenti di Firenze) senza che mi fosse lasciato alcun segno di riconoscimento.

Una sola volta - quindici anni or sono - ho fatto ricerche presso l'Asilo intorno alla mia paternità e soltanto allora ho saputo che, nato di pochi giorni, fui dato a balia ad una famiglia di piccoli possidenti delle montagne pistoiesi presso i quali rimasi fino all'età di quattro anni.

In un giorno dell'estate del $1902 \mathrm{mi}$ recai a Prunetta a trovare il mio primo tenutario (Giovanni Gavazzi ottantenne ancora vivente) da cui appresi di essere io nato dall'unione clandestina di un principe con una governante della Casa Reale.

Questa notizia non mi rallegrò, all'opposto mi accese di pietà e di vergogna e da quel giorno mi decisi a non fare ulteriori indagini sulla mia provenienza?

In realtà il bambino era stato portato all'ospedale il 19 ottobre (non il giorno successivo), l'origine nobile non era in alcun modo dimostrata e probabilmente non rimase per quattro anni sempre presso la famiglia Gavazzi. Ma, soprattutto, Targioni avrebbe continuato a fare 'indagini' per tutta la vita e sarebbe tornato agl'Innocenti - sicuramente a metà degli anni Venti e forse anche altre volte -, dove però non venne mai informato dell'esistenza di un segno di riconoscimento: evidentemente, il personale dell'istituto rispettava le procedure previste verso i trovatelli; nel 2018, invece, ho potuto vedere e fotografare il segnale e il suo contenitore (Fig. 2).

E però vero che Targioni lasciò il «caro villaggio di montagna» ${ }^{10}$ dei Gavazzi il 7 novembre 1872; quel giorno il bambino fu «rimesso in casa», ovvero venne riportato all'Ospedale degl'Innocenti ${ }^{11}$. Non conosciamo i motivi del nuovo abbandono, ma probabilmente è vera la ricostruzione fornita da Edelberto, quando oramai si faceva chiamare Idalberto, «correva l'anno 1872 , quando il mio tenutario fu costretto ad emigrare insieme ad alcuni suoi figli maggiori ed ebbe a ricondurmi all'Asilo. Come abbiamo veduto, io allora contavo solo quattro anni $\gg^{12}$.

Targioni narra di avere incontrato, nei giorni successivi, la madre («governante di Casa Reale») che, costretta dal marito (il «principe») ad abbandonare nuovamente il figlio, lo «baciò per un'ultima volta». L'episodio avrebbe originato in Targioni una «profonda repugnanza per tutta l'aristocrazia e per

\footnotetext{
9 Ivi, pp. IX-X.

10 AIT, Scatola 3.31, Cinquant'anni, cit., p. 12.

11 AOIF, Balie e Bambini 1868, cit.

12 AIT, Scatola 3.31, Cinquant'anni, cit., p. 16; cfr. anche Idalberto Targioni, Canzoniere di poesie sociali, cit., p. X.
} 
quei piccoli parassiti che le stanno d'intorno a inchinarla e servirla compiendo un lavoro improduttivo che avvilisce ed abbassa la dignità umana $\gg^{13}$. In effetti, l'ostilità verso l'aristocrazia del Targioni socialista possiamo verificarla con certezza ${ }^{14}$, ma il presunto incontro con la madre naturale non si è potuto accertare.

Dopo appena una settimana dalla partenza da Casa Marconi e il rientro agl'Innocenti, il 14 novembre 1872 Idalberto fu affidato «a Giuditta [Giraldi] moglie di Domenico Capecchi del Popolo di San Baronto» ${ }^{15}$, una famiglia di contadini proprietari di «un magro campicello» ${ }^{16}$ e residenti in una casa di una frazione rurale del Comune di Lamporecchio, sul crinale del Montalbano tra Empoli e Pistoia, che nel 2018 conservava sulla facciata una targa in onore al poeta contadino: «Qui Idalberto Targioni cedendo l'umile fatica alla terra madre ne traeva l'ispirazione per l'improvvisa, schietta poesia» (Fig. 3) ${ }^{17}$. Oggi l'edificio, in via Sanbarontana, è in ottimo stato; con una bella vista sulla valle, ospita due appartamenti. Ma all'epoca quella frazione appariva a Targioni come

un povero villaggio tagliato fuori dal mondo, lontano 18 chilometri dalle più prossime stazioni ferroviarie, privo d'industrie e di commerci, al quale si accedeva per vie rapide e malagevoli, con agricoltura primitiva e con poche case coloniche sparse qua e là per le vallate e sui colli ricoperti di viti e olivi, piccole, basse, dalle finestre senza vetri, coi tetti giallastri e le mura bigie prive di grazia e d'intonaco che narravano all'osservatore tutta una storia secolare di un popolo non ancora pervenuto ai primi inizi di una nuova civiltà. Se per colpa e ignoranza di quelli che le fecero costruire, in quelle case, spesso umide, era penuria di aria e di luce, non meno vi si trascurava l'igiene e la pulizia per colpa e per ignoranza di coloro che le abitavano. Buche larghe e profonde, aperte, e scoperte e alte masse di concio di stalla sorgevano in luogo del cesso al contatto delle mura e sotto le basse finestre dalle quali venivano gettati di continuo gli escrementi ed altre immondizie.

Più in alto, selve d'annosi e folti castagni, di antiche e orride querce, di vaste e profonde boscaglie di cerro e di stipa. Ecco quale appariva in quell'epoca il territorio di S. Baronto, una delle frazioni del Comune di Lamporecchio ${ }^{18}$.

In quella casa «brutta e triste tanto che da piccolo non voleva mai rimanervi solo», tutti dovevano lavorare per una economia di sussistenza che la-

13 Targioni, Canzoniere di poesie sociali, cit., pp. XI-XII.

${ }^{14}$ Cfr., ad esempio, I. Targioni, Piccoli parassiti: Opuscolo di propaganda, Tip. U. Polli, Firenze 1912 .

15 AOIF, Balie e Bambini 1868, cit. Per il cognome da nubile di Giuditta Giraldi Capecchi cfr. Martini, In memoria, cit., p. 8.

16 AIT, Scatola 3.22, Idalberto Targioni. Educatore e organizzatore delle nostre masse rurali e contadine.

${ }_{17}$ Ringrazio Roberta Niccoli, Daniele Bartalucci e Monica Cetraro, assessore alla cultura di Lamporecchio, per la fotografia.

18 AIT, Scatola 3.31, Cinquant'anni, cit., p. 18, alcuni refusi sono stati corretti nella trascrizione. 
sciava pochi margini di accesso al mercato e il piccolo Idalberto fu coinvolto fin da subito nei molteplici mestieri che caratterizzavano il lavoro contadino, tra campi e case. Idalberto soggiornò di nuovo agli Innocenti tra il 20 ottobre e il 17 novembre 1876 per una tonsillite, senza però essere operato ${ }^{19}$. Ma ormai la sua vita sembrava potersi svolgere sul Montalbano.

Come altri figli di contadini, mezzadri e pigionali, non andò a scuola - la legge Coppino fu varata nel 1877, quando aveva 9 anni -, ma imparò a leggere un po' da solo e un po' grazie all'aiuto di un adulto, che per Targioni fu un prete. «Ma il Sig. Curato non aveva troppo tempo da perdere e nemmeno di dar lezione»; in seguito, da autodidatta, imparò anche a scrivere. La crescita intellettuale fu rapida, visto che tra gli otto e i dieci anni già decantava versi e scriveva persino le sue prime rime ${ }^{20}$.

Non si trattava del frutto geniale di un fiore isolato; i racconti, le parole in versi, il recitar cantando, le rime in ottava erano parte integrante della cultura popolare trasmessa nelle veglie di fronte ai camini, nei campi durante i lavori agricoli o nelle feste di paese. Forse ancor di più nella Lamporecchio che a fine Quattrocento aveva dato i natali a Francesco Berni ${ }^{21}$.

Però Targioni seppe coltivare più di altri la passione poetica e farsi «una discreta cultura dovuta esclusivamente alla sua volontà di istruirsi», come avrebbero sottolineato gli informatori di polizia alcuni anni dopo ${ }^{22}$. Era un piccolo adolescente quando divenne un apprezzato e abile stornellatore estemporaneo tra i suoi borghi e le campagne dintorno. «I contadini del vicinato, fin dove poteva giungere la mia voce, appoggiati al manico della vanga, immobili come statue, restavano lunghe ore inoperosi per sentire il Bastardo (così mi chiamavano) cantar di poesia! $\gg^{23}$.

In Toscana - avrebbe scritto Targioni anni dopo - ogni villaggio vanta il suo poeta estemporaneo, per lo più nel ceto agricolo ed artigiano. Con molti di loro mi battevo allora riportando spesso la palma. A dodici anni sapevo a memoria tutto l'Inferno e molt'altre cantiche della Divina Commedia ${ }^{24}$.

19 Ivi, p. 30; cfr. AOIF, Balie e Bambini 1868, cit. Sul male alle tonsille, che lo avrebbe accompagnato per molti anni, cfr. AIT, Scatola 3.31, Cinquant'anni, cit., p. nn.

20 AIT, Scatola 3.31, Cinquant'anni, cit., p. 40.

${ }^{21} \mathrm{Su}$ questi aspetti si possono consultare i testi e le pubblicazioni richiamate in $<$ http://www. accademiadellottava.it〉, e in «Aporie», <https://goo.gl/M7DzhH>. Per orientarsi cfr. $I$ canti della tradizione popolare toscana. Bibliografia, Regione Toscana, Firenze 2017. Per una introduzione al tema cfr. A. Bencistà, L'ambulante scuola. Breve storia dell'ottava rima in Toscana, Pupi e Fresedde, Regione Toscana, 2005, <http://www.pannostrale.it/improvvisatori. html>. Si veda anche Id., Idalberto Targioni, «Toscana folk», III (4), 1999, pp. 32-34.

${ }^{22}$ Archivio centrale dello Stato (ACS), Casellario politico centrale (CPC), b. 5032, f. 105270, Targioni Idalberto, estremi cronologici 1909-1942, Cenno biografico al giorno 14 gennaio 1909.

${ }_{23}$ Targioni, Canzoniere di poesie sociali, cit., p. XIII, corsivi nel testo.

${ }^{24}$ AIT, Scatola 3.31, Cinquant'anni, cit., p. 42. 


\section{L'Usignolo: l'adolescenza}

In conflitto con Domenico Capecchi, Targioni fu minacciato più volte dal padre adottivo di essere riportato all'istituto o affidato ad altre famiglie anche perché, avrebbe ricordato Idalberto, «i rimproveri e le busse non valsero a niente anzi mi rendevano più caparbio e disobbediente ${ }^{25}$. Infatti, a 12 anni il ragazzino fu riconsegnato agl'Innocenti, dove incontrò nuovamente «quegli uomini misteriosi in abito nero», visti anni prima, e ne dedusse che «erano dei preti», forse gli stessi che avevano dato al bambino il senso di peccato e di espiazione. «Babbo Domenico fece loro un lungo discorso», «un vero atto d'accusa contro di me che però sentivo di non approvare», avrebbe in seguito ricordato Targioni.

Fui severamente rimproverato e minacciato di non so quali castighi, perché secondo quanto mi contestavano quegli uomini in abito nero ero stato un cattivo, un disobbediente, un caparbio e senza voglia di lavorare.

Intanto mi si facevano queste ammonizioni, fu tirata una corda e tosto s'aperse una porta da cui vidi uscire la vecchia e grassa Matilde. Sentii come un tuffo al cuore e subito riconobbi in quella donna la rigida bambinaia bigotta che mi aveva imprigionato tante volte nello stanzino e costretto a segnare colla lingua molte croci sul pavimento. Mi venne ingiunto di seguirla: obbedii mezzo stupito dal dolore e da un profondo senso di terrore che si era impossessato di me.

[...] Là dentro nessuna cosa aveva cangiato aspetto o stile. Ancora l'antica immobilità, la stessa atmosfera pesante, grigia e monotona, le solite orazioni, la stessa clausura, fanciulli di otto, di dieci, di dodici e più anni che come prima giungevano da vari luoghi e partivano per ignota destinazione. Un mese circa rimasi in questa specie di casa di correzione ${ }^{26}$.

Nelle memorie, Targioni narra di essere stato successivamente preso in consegna da «un uomo sulla cinquantina, decentemente vestito, dalla barba lunga e nera e dall'aspetto molto severo»; a suo dire, era un certo Angiolo Romagnini che viveva nei pressi di Reggello, un Comune della Provincia di Firenze. Il bambino lasciò gli Innocenti con colui «che la voce pubblica nel suo paese lo aveva battezzato col nome di "Piccolo mercante di carne umana" ${ }^{27}$ - avrebbe scritto il Targioni cinquantenne - e dopo breve tempo l'uomo barbuto consegnò Idalberto a un contadino di Tosi (una frazione di Reggello a circa $7,5 \mathrm{~km}$ in linea d'aria dal capoluogo, circa $23 \mathrm{~km}$ a est di Firenze, ovvero a una cinquantina di $\mathrm{km}$ a volo d'uccello dalla casa di San Baronto Baronto a Lamporecchio) in cambio di 50 lire:

25 Ivi, p. 44.

26 Ivi, pp. 49-51.

27 Ivi, p. 52 e p. nn. (ma p. 61). 
da questo breve aneddoto - si legge nelle memorie del 1920 - il lettore rileverà come noi poveri trovatelli eravamo messi a prezzo simili a bestie sul mercato. Nessun amore, nessuna pietà muoveva coloro che ci avevano richiesti. Il loro scopo era di farci lavorare il più possibile per trarne il massimo utile col minore dispendio. La schiavitù, che si diceva e si dice abolita riviveva in noi sotto nuova forma non però meno delle altre ipocrita e vile ${ }^{28}$.

Queste considerazioni, dunque, sarebbero state scritte molti anni dopo i fatti rievocati, da un Targioni con oramai molta esperienza politica alle spalle, però possono farci capire lo stato d'animo e le rielaborazioni della memoria di quel ragazzino divenuto adulto, oltre ad aprire qualche squarcio su un mondo oggi dimenticato ${ }^{29}$.

In effetti, nel registro degli Innocenti non si fa menzione di Angiolo Romagnini, o di altri contadini ricordati nelle memorie, ma si trova solo annotato che il 2 febbraio 1882 il trovatello era stato riportato all' istituto dai Capecchi «perché non voleva fare il contadino» e che il giorno dopo, 3 febbraio, veniva consegnato «all'Anna moglie di Giuseppe Sacconi del Popolo di S. Andrea a Tosi del Comune di Reggello» ${ }^{30}$. Qui rimase appena 16 mesi: un dato che coincide con le memorie di Targioni, dove si narra di un soggiorno «di oltre un anno» nella casa di Tosi. Non sappiamo in quale mese, ma sembra certo che nell' inverno 1882-1883 «riportò una lieve condanna per furto semplice» ${ }^{31}$.

Nuovamente nel «1883 al dì 26 giugno fu rimesso nello Spedale perché non voleva fare il contadino ${ }^{32}$. All'arrivo, «il solito signore» dell' istituto lo avrebbe accolto dicendo «Ancora qui tu? Non hai trovato un busto che t'entri? Eppure dovrai fare quello che fanno gli altri: Se tu vuoi che il mento balli / Alle mani facci i calli», ovvero: 'se vuoi mangiare, devi lavorare sodo' ${ }^{33}$. Il 3 luglio 1883, finalmente, il quindicenne venne riconsegnato ai Capecchi di Lamporecchio, da «mamma Giuditta», e da allora in poi non sarebbero più state registrate notizie a suo riguardo nelle carte degli Innocenti ${ }^{34}$.

Un paio di anni dopo, nel pieno di un'adolescenza turbolenta fatta di fughe e piccoli furti, gioco d'azzardo e debiti, episodi violenti e ostilità verso isti-

${ }^{28}$ Ivi, p. nn. (ma p. 60).

29 Sulla schiavitù in età contemporanea cfr. G. Turi, Schiavi in un mondo libero. Storia dell'emancipazione dall'età moderna a oggi, Laterza, Roma-Bari 2012.

${ }^{30}$ AOIF, Balie e Bambini 1868, cit.

${ }^{31}$ Lo scrisse il Direttore della DGPS Giacomo Vigliani al Procuratore generale federale, Berna, il 2 maggio 1912: ACS, CPC, b. 5032, cit. Nelle memorie narra di tre giorni di carcere per furto di legna durante l'inverno (con un amico stava segando rami di abeti quando fu preso dal proprietario), che sarebbero stati scontati a 13 anni, quindi nel 1881 e non nel 1883 come invece si legge nelle carte del CPC; cfr. AIT, Scatola 3.31, Cinquant'anni, cit., p. nn., dove si trova anche la citazione sul periodo a Tosi.

${ }^{32}$ AOIF, Balie e Bambini 1868, cit.

${ }^{33}$ AIT, Scatola 3.31, Cinquant' anni, cit., p. nn., dove si legge che all'epoca aveva 14 anni.

${ }^{34}$ Cfr. AOIF, Balie e Bambini 1868, cit. L'agognato incontro con l'amata Giuditta e il viaggio da Firenze a San Baronto è narrato in AIT, Scatola 3.31, Cinquant'anni, cit., pp. nn. 
tuti, istituzioni, autorità e clero, a 17 anni Targioni lasciò la campagna «con due lire in tasca, un sacchetto di cenci, i miei libri e tanto da vivere un giorno». Prima di partire scrisse una lettera alla mamma adottiva:

Le raccontai come aveva [sic] perduto al gioco tutto il danaro ricevuto dal Babbo la mattina precedente e che, disperato e sopraffatto dal rimorso e dalla vergogna, aveva deciso andarmene volontariamente e lavorar fuori come a punirmi dei falli commessi. Le domandavo perdono e promettevo scriverle appena giunto al luogo della nuova residenza e concludevo assicurandola che avrei fatto ogni sforzo per avanzarmi tanto da saldare i miei debiti e ritornare a lei, dopo provato il morso del lupo, buono e laborioso come una volta, bel calzato e vestito. Così ancora una volta in compagnia de' miei poeti, alcuni stracci e pochi alimenti, abbandonai l'umile e povera casetta ${ }^{35}$.

Tra 1885 e 1891 passò sei anni lontano da Lamporecchio. Lavorò come manovale e terrazziere alla costruzione della linea ferroviaria Firenze-Faenza, nei pressi delle Caldine (Fiesole), poi svolse il servizio di leva a Casale Monferrato in Provincia di Alessandria, trovando «subito la vita della caserma qualche cosa da assomigliarsi ai lavori forzati e mi considerai - scrisse Targioni - come un condannato ingiustamente dalla legge ad una pena che non avevo meritato ${ }^{36}$. A 23 anni, nel 1891, tornò a San Baronto e riprese il lavoro della terra con la famiglia adottiva. Quel periodo lontano da casa fu formativo e accelerò la sua crescita; la conoscenza diretta delle ingiustizie sociali che dominavano anche nelle città, nel mondo del lavoro e nell'esercito favorì l'incontro con la politica e col socialismo negli anni della Seconda internazionale, che offrirono risposte al suo desiderio di riscatto e una prospettiva di impegno politico praticabile rivolto al futuro. Per quasi un lustro fece il contadino, il poeta-contadino, il lettore di opere che contribuivano ad arricchire la sua ancor piccola biblioteca, l'apprendista del socialismo ${ }^{37}$.

\section{Il Diavolo rosso: poeta, anticlericale, socialista}

Quando, nel 1895, Targioni pubblicò il suo primo Canzoniere di poesie popolar ${ }^{38}$ aveva 27 anni. Le prime produzioni edite erano di ispirazione idillica, «l'amicizia, i pensieri, la natura, ecc. sono gli argomenti che tratta», avrebbe osservato Libertario Guerrini sessant'anni dopo ${ }^{39}$, ma erano anche di ispirazione religiosa come mostrano i versi pubblicati nello stesso anno per $\mathrm{Il}$

\footnotetext{
35 AIT, Scatola 3.31, Cinquant'anni, cit., pp. nn.

${ }^{36}$ Ivi, pp. nn.

37 Cfr. ivi, pp. nn.

38 Pistoia, Tip. Flori e Biagini.

39 Guerrini, Un poeta estemporaneo, cit., p. 512.
} 
terribile terremoto: «A te ricorro, sommo Ente Divino, che il mondo a voglia tua regoli e guidi [...] Vi consiglio perciò, cari uditori, a più non provocar la sua vendetta, ma torni a penitenza i vostri cuori, che Iddio lo brama e paziente aspetta $\gg^{40}$.

Negli stessi mesi si iscrisse al Partito socialista - fondato nel 1892, il Partito dei lavoratori italiani aveva assunto la nuova denominazione proprio nel 1895 -, con l'intenzione di dedicarsi interamente a una militanza che l'avrebbe allontanato dai campi, ma non dal lavoro dei contadini e dalle loro condizioni di vita. L'anno successivo, il 9 dicembre 1896, si sposò con Amelia Vannacci, una contadina nata a Lamporecchio sette anni dopo di lui, sorella di Afrisio - un uomo che avrebbe fatto un po' di carriera con un matrimonio ben riuscito e come fattore presso la fattoria di Papiano e che sarebbe divenuto podestà di Lamporecchio nel 1943, mantenendo l'incarico con la Repubblica Sociale Italiana fino alla Liberazione. Idalberto e Amelia ebbero due figlie: Velia, nata prima del matrimonio, e Rita; la seconda sarebbe poi morta a nove anni per una grave malattia alle ossa (Fig. 4$)^{41}$.

Il matrimonio cadde negli anni della repressione dei Fasci siciliani (1894), delle lotte delle trecciaiole in Toscana (1895), della sconfitta italiana ad Adua (1896), delle prime esperienze di municipalismo socialista in Italia e poi anche in Toscana, dove il Psi conquistò il Comune di Colle Val d'Elsa nel 1897, lo stesso anno in cui Giuseppe Pescetti diveniva il primo deputato socialista della regione ${ }^{42}$.

Non a caso, dunque, fu in quel periodo che le sue poesie scritte o improvvisate si piegarono decisamente verso la denuncia delle ingiustizie che dominavano il mondo rurale e a favore della diffusione degli ideali socialisti tra i lavoratori della terra. I contrasti, i contraddittori in rima, le 'accademie in versi' pubbliche e private di Targioni godettero di un ampio successo tra le classi rurali di Lamporecchio e di tutta l'area del Montalbano, dove il bacino di influenza del nuovo socialismo iniziò a radicarsi incrinando l'egemonia

${ }^{40}$ I. Targioni, Il terribile terremoto a Firenze e nei paesi circonvicini. Narrazione dell'estemporaneo Idalberto Targioni, Tip. Salani, Firenze 1895. Sull'evento sismico, che causò danni notevoli e alcuni morti, cfr. E. Cioppi, 18 maggio 1895: storia di un terremoto fiorentino, Osservatorio Ximeniano, Firenze 1995.

${ }^{41}$ Amelia era nata il 25 luglio 1875 e sarebbe morta nel 1968. Velia (nata il 20 marzo 1894 e morta nel 1979) si sarebbe poi sposata con Torello Chiappini (1890-1985) ed ebbe tre figli: Rita il 31 agosto 1914 (morì nel 1936), Lida nel 1917 e nel 1920 Idalberto, il padre di Simonetta Chiappini che mi ha fornito queste notizie nei colloqui del 12 e 22 maggio 2018. Cfr. AIT, Scatola 2, Al mio carissimo cognato Afrisio Vannacci nella fausta occasione delle sue nozze colla signorina Elide Torrigiani in segno d'affetto e stima perenni offro, cartolina a stampa, Tip. Cooperativa, Pescia 22 ottobre 1904; Poli, Lamporecchio, cit., p. 71.

42 Per orientarsi rinvio a E. Fasano Guarini, G. Petralia, P. Pezzino, Storia della Toscana, vol. 2: Dal Settecento a oggi, Laterza, Roma-Bari 2004; G. Mori, Dall'unità alla guerra: aggregazione e disgregazione di un'area regionale, in Storia d'Italia dall'Unità a oggi. Le regioni. La Toscana, cit., pp. 247 sgg.; su Pescetti si può vedere Bianchi, Pescetti Giuseppe, in Dizionario Biografico degli Italiani, cit., vol. 82, 2015, pp. 603-605. 
conservatrice, cattolica e clericale su quelle campagne. Le fiere e i mercati nelle piazze dei paesi erano occasioni preziose per incontrare in modo regolare paesani, mezzadri e lavoratori rurali che seguivano con entusiasmo i componimenti poetici. L'importanza della «figura tradizionale del poeta popolano, del cantastorie» nel «tener desto lo spirito di associazione e di lotta nelle campagne» fu rilevata in sede storica da Giuliano Procacci molti anni fa ${ }^{43}$. In effetti, riallacciandosi a una tradizione antica, i socialisti sfruttarono questi luoghi per divulgare poesie a carattere sociale e politico, sostenendo a tal fine poeti estemporanei come Targioni. Già soprannominato il «Bastardo» e poi l'«Usignolo» (o «Rosignolo»), col crescere delle tensioni con le élite clericali e liberali che dirigevano il Municipio di Lamporecchio Idalberto fu ribattezzato il «Diavolo Rosso» dai «preti della sua campagna» ${ }^{44}$. Nel maggio 1898, in seguito alla repressione dei moti popolari e nel pieno della 'crisi di fine secolo', conobbe «per la prima volta le delizie delle manette e del carcere passando qualche mese alle Stinche di Pistoia» ${ }^{45}$. Ma l'esperienza di prigionia non rallentò l'opera di proselitismo e Targioni intensificò la sua attività politica.

Nel 1901 venne eletto consigliere socialista al Comune di Lamporecchio, in una lista popolare che comprendeva anche il suo amico Domizio Torrigiani, di otto anni più giovane (1876-1932). Quella di Torrigiani fu una figura importante per Targioni, ma forse non tanto quanto quella di Targioni per Torrigiani. Possidente, proprietario anche di una casa a Lamporecchio in località Il Muro, di una villa a San Baronto in località Al Poggio, vicino alla casa dei Capecchi, e di alcuni poderi, anche Domizio Torrigiani era fortemente anticlericale e laicista, tanto da aver aderito a una loggia massonica empolese intorno ai vent'anni. Avvocato, negli anni Dieci dirigente del Partito radicale italiano e presente sulla scena politica fiorentina (nella città dove la sua famiglia si era trasferita a inizio secolo, senza recidere i profondi legami col Montalbano), interventista come Targioni e volontario di guerra, Torrigiani ottenne nel 1916 il XXXI grado della gerarchia scozzesista. Nel giugno 1919 avrebbe svolto il ruolo di patrono del Comitato per l'erezione di un monumento ai Caduti nella piazza di Lamporecchio presieduto da Targioni e, dal 23 di quel mese fino alla dissoluzione della libera muratoria nella Penisola, sarebbe stato Gran maestro del Grande Oriente d'Italia, la più importante obbedienza massonica italiana con sede a Palazzo Giustiniani, a Roma. La solida amicizia fra Targioni e Torrigiani durò a lungo, ma il cammino comune di questi due uomini notevoli di

43 Giu. Procacci, La lotta di classe in Italia agli inizi del secolo XX (ed. orig. 1970), Editori Riuniti, Roma 1992, p. 142. Sull'affermazione del socialismo prima del 1914 cfr. D. Sassoon, Cento anni di socialismo. Le sinistre nell'Europa occidentale del XX secolo, Editori Riuniti, Roma 1997 (1996), pp. 5-30.

${ }^{44}$ Guerrini, Un poeta estemporaneo, cit., p. 512.

45 I. Targioni, Vent'anni di propaganda e cinque anni d'Amministrazione Socialista nel Comune di Lamporecchio, Tip. Guido Grazzini, Pistoia 1920, p. 3. 
Lamporecchio si sarebbe interrotto a metà anni Venti, durante l'offensiva mussoliniana contro la massoneria, che costrinse Torrigiani all'esilio, al confino e poi all' isolamento nella villa di San Baronto - già presa di mira dalla 'seconda ondata' squadrista -, fino alla morte avvenuta due mesi prima del decennale della Marcia su Roma e un paio d'anni dopo quella di Targioni ${ }^{46}$.

Intanto, nel 1902, Targioni aveva pubblicato un canzoniere di poesie sociali ${ }^{47}$ con testi che mostrano la sua vicinanza a un certo socialismo umanitario tanto distante da Marx quanto permeato da dottrine positiviste ed evoluzioniste, nonché spiccatamente influenzato dal laicismo e da un anticlericalismo di matrice risorgimentale: elementi che, con molti altri, concorrevano a costituire i caratteri peculiari di quella sorta di 'commonwealth ideologico' che avrebbe caratterizzato il Psi, le sue anime dissonanti e le sue tensioni interne fino all'ascesa fascista. Come mostra anche il successivo inno all' Avvenire, i testi di Targioni avrebbero continuato a intrecciare elementi di spiritualità cristiana con temi internazionalisti:

Tutta la gente oppressa la verde erta spinosa del monte doloroso salga. Le splende il sole dell'avvenire in fronte! Salga, salga sul vertice della montagna altera! Lassù, saluta i popoli una rossa bandiera che s'agita alle libere aure del ciel di maggio; all'ombra di quel labaro, del sole al mite raggio, benedirem la pace, maledirem la guerra, inneggeremo al regno della giustizia in terra ${ }^{48}$.

Nello stesso anno Idalberto fu tra i fondatori della sezione socialista di Lamporecchio e tra i principali promotori di una mobilitazione dei mezzadri per la revisione dei patti colonici. Era necessaria «una politica fresca», concreta e alternativa a quella delle «"vecchie cariatidi" che fanno del Comune un uso privato», capace di dare corpo a richieste concrete:

Miglioramento graduale dei servizi pubblici, esecuzione dei lavori di improrogabile necessità, un maggiore stanziamento di fondi per l'ospedalità e la beneficenza, maggiore vigilanza alle scuole, miglioramento del servizio igienico sanitario, riforma tributaria su base progressiva ${ }^{49}$.

${ }^{46}$ L'Archivio Domizio Torrigiani (ADT) è conservato presso l'Istituto Storico Toscano della Resistenza e dell'Età contemporanea (ISRT); nella Serie I Carteggio, I.20.1, il fondo possiede un f. con un paio di lettere di Targioni a Torrigiani. Cfr. Archivio Domizio Torrigiani, inventario a cura di Eugenia Corbino, ISRT, Firenze 2012; Francini, Balli, Il "gran maestro" Domizio Torrigiani, cit., pp. 22-26, 75-76, 108-109; L. Cerasi, Democrazia del lavoro, laicismo, patriottismo: appunti sulla formazione politica di Domizio Torrigiani, in La massoneria italiana da Giolitti a Mussolini, cit., p. 14; Bianchi, Grande guerra, Grande dopoguerra, cit., pp. 272-273; Id., Massoneria e fascismo, cit., pp. 124 e 135. Sulle proprietà Torrigiani cfr. Poli, Lamporecchio, cit., ad nomen.

${ }^{47}$ I. Targioni, Il canzoniere: poesie sociali, Tip. Traversari, Empoli 1902.

48 I. Targioni, L'avvenire: canto epico del poeta estemporaneo Idalberto Targioni, Tip. Traversari, Empoli 1905, pp. 6-7.

49 Targioni, Vent'anni, cit., p. 4; Poli, Lamporecchio, cit., pp. 62-63. F. Conti, Dalla sociabilità d'élite all'associazionismo di massa: società civile e riti della modernità a Pistoia nell'età libera- 
Nel 1903 venne eletto segretario della Camera del Lavoro di Pistoia e per un certo periodo abitò in città, al numero 3 dello Sdrucciolo della Sapienza, come si legge in una lettera inviata a Domizio Torrigiani, nei pressi delle attuali sedi dell'Archivio di Stato e della Biblioteca Forteguerriana ${ }^{50}$.

Furono anni di intensa attività politica, sindacale e poetica per l'energico Targioni che ritroviamo, ad esempio, a Mammiano (una frazione attualmente appartenente al Comune di San Marcello Piteglio, sull'Appennino Pistoiese) nel 1904 - chiamato a inaugurare la società operaia Giuseppe Garibaldi ${ }^{51}$-, mentre iniziava a essere schedato dalle forze di polizia come attestano i documenti conservati presso il Casellario politico centrale (CPC), e che pure non esitava a tessere relazioni extraconiugali. Il rapporto con la moglie Amelia si incrinò, divennero frequenti le relazioni con altre donne e probabilmente ebbe un figlio non riconosciuto: il Renzo che per un certo periodo avrebbe vissuto con Tranquillo Gasperini, suo padre tutore, e che avrebbe avuto un fitto scambio di lettere con Targioni nel corso degli anni Venti ${ }^{52}$.

Nel 1909, mentre «pendeva a suo carico procedimento penale per diffamazione a mezzo della stampa $\gg^{53}$, Targioni conobbe a Firenze Sofia Bacarelli di Poggio a Caiano (un centro noto per la Villa medicea, collocato sull'ultima propaggine del Montalbano protesa verso Prato, lungo la strada tra Firenze e Pistoia), con la quale imbastì una solida relazione che lo portò a trasferirsi in un modesto appartamento nei pressi del mercato di San Lorenzo a Firenze, sfidando l'ostilità dei genitori della Bacarelli. Nel 1911 Idalberto e Sofia ebbero un figlio, morto pochi giorni dopo il parto, e nel 1916 una figlia, Idalberta (Albertina, Fig. 5). La doppia vita, non clandestina, lo avrebbe costretto a seguire la nuova famiglia fiorentina e quella rimasta a Lamporecchio, costringendolo a fare la spola tra il capoluogo (nel 1915 si trasferì con la Bacarelli al Galluzzo, nei pressi della Certosa di Firenze) e il paese per gran parte della sua vita, e anche a seguire le vicende di Renzo, il suo probabile primo figlio non riconosciuto ${ }^{54}$.

le, in G. Petracchi (a cura di), Storia di Pistoia, vol. IV, Nell'età delle rivoluzioni, 1777-1940, Le Monnier, Firenze 2000, p. 541. Cfr. anche S. Neri Serneri, Il localismo come vincolo e come risorsa. Note sul caso toscano, in I. Milanese (a cura di), Le Camere del lavoro italiane. Esperienze storiche a confronto, Longo, Ravenna 2001, pp. 191-207.

${ }^{50}$ ISRT, ADT, Serie I Carteggio, I.20.1, Targioni a Torrigiani, 16 settembre 1904. Nella lettera lo scrivente fa riferimento all'inaugurazione di una epigrafe a Lamporecchio.

${ }^{51}$ Cfr. L. Savelli, L'industria di montagna. Uomini e donne al lavoro negli stabilimenti della Società Metallurgica Italiana, Olschki, Firenze 2004, p. 120.

52 Cfr. AIT, Scatola 2.7.14-15, e soprattutto AIT, Scatola 4.3.20 Corrispondenza di Renzo.

53 ACS, CPC, b. 5032, cit., Direttore DGPS Giacomo Vigliani a Procuratore generale federale, Berna, 2 maggio 1912.

${ }^{4}$ Si veda la corrispondenza di Targioni con la figlia Velia, la moglie Amelia e la compagna Sofia in AIT, Scatola 4; ivi, Scatola 2.23.31, Bozza di poesia dedicata a Sofia Bacarelli. Su Idalberta cfr. ivi, Scatola 2.19.1, Contemplando la mia Idalbertina a 22 mesi mentre dorme, 
Intanto Targioni dispiegava la sua produttività poetica e letteraria. Tra il 1895 e il 1912 pubblicò più di 20 opere, senza rinunciare alle improvvisazioni e ai canti in ottava rima ${ }^{55}$. In quel periodo svolse anche una fitta attività pubblicistica, collaborando con vari periodici socialisti, e in particolare con «La Martinella» di Colle Val d'Elsa (dal 1899 al 1901), «L'Avvenire» di Pistoia (1901-1915) e «Vita Nuova» di Empoli (1901-1915), assumendo a più riprese l'incarico di direttore. Nel 1908 fondò «Il Risveglio del Montalbano», poi divenuto «Risveglio della plebe» e infine confluito ne «L'Avvenire», che diresse fino al 1912; nel $1911 \mathrm{fu}$ anche direttore del bollettino $\ll \mathrm{La}$ vigna nuova $»^{56}$. Nel 1913 si candidò alle elezioni per la Camera dei deputati al Collegio Pistoia $\mathrm{I}^{57}$.

Quello fino al $1914 \mathrm{fu}$ il periodo d'oro del Targioni cantore, sindacalista e socialista, che alternava contraddittori, accademie in versi e dibattiti politici nelle locande, nelle piazze e nei circoli di gran parte della Toscana, ma anche

in Quaderno Poesie. Idalberta Bacarelli non si sarebbe sposata e non ebbe figli. Cfr. anche Targioni «traditore» per amore, «Il Tirreno», 23 giugno 1996.

${ }^{55}$ Cfr. Canzoniere di poesie popolari, cit.; Il terribile terremoto a Firenze, cit.; Il canzoniere: poesie sociali, cit.; Contrasto tra un prete democratico-cristiano e un contadino ascritto alla Lega di resistenza, Sez. socialista di Empoli, Empoli 1902; Terza risposta del Targioni al Manuelli, Tip. E. Severi, Firenze 1902; Caprera: La casa e la tomba di Giuseppe Garibaldi: terzine, Tip. Niccolai, Pistoia 1904; L'avvenire: canto epico, cit.; Il prete attraverso le religioni e la storia: poemetto eroicomico in ottava rima del poeta estemporaneo Idalberto Targioni, Tip. U. Polli, Firenze 1906; Martiri del lavoro: sestine, Ciattini, Pistoia 1906; Gli scandali clericali, Tip. Cooperativa, Pescia 1907; L'incendio della miniera: Bozzetto sociale in versi, Tip. I. Cecchi e C., Prato 1908; Agli emigrati italiani, Tip. L. Migliorini, Zurigo 1908; Dalla nascita alla morte della terra: Conferenza in versi, tenuta la prima Volta all'Università popolare di Zurigo il 10 Maggio 1909, Tip. Fratelli Ciattini, Pistoia 1909; Il terremoto calabro-siculo: Bozzetto sociale in versi martelliani, Tip. Fratelli Ciattini, Pistoia 1910; Civiltà borghese o tirannide capitalistica? Terzine, Tip. U. Polli, Firenze 1911; Risposta per le rime alla canzone d'oltremare di Gabriele d'Annunzio, Tip. U. Polli, Firenze 1911; Per l'educazione della donna secondo la morale cristiana e socialista: contradittorio in ottava rima tenuto dai due poeti improvvisatori Fortunato Chiti e Idalberto Targioni la sera del 19 marzo 1911 nella Società di M. Soccorso di Varlungo (Firenze), resoconto stenografico, Tip. Polli, Firenze 1911; I miei Ricordi d'infanzia, cit.; Piccoli parassiti, cit.; Canzoniere di poesie sociali, cit.; Ettore Giovannitti e l'emigrazione transoceanica, Tip. Ugo Polli, Firenze 1912.

56 Cfr. «La vigna nuova: sistema pratico di coltivazione della vite a grande espansione ritrovato da Vincenzo Guerrazzi», a. I, n. 1, 20 marzo 1911. Targioni collaborò in modo meno regolare anche con altri periodici, come «Il Risveglio», settimanale di Grosseto. Cfr. F. Boldrini, Minatori di Maremma. Vita operaia, battaglie sindacali e lotte politiche a Ribolla e nelle Colline metallifere (1860-1915), introduzione di I. Tognarini, Comune di Roccastrada, Roccastrada 2006, p. 261; G. Perugi, Alle urne! Le elezioni politiche del 1913 nelle cronache dei giornali locali, in E. Galli, G.C. Romby (a cura di), Voci di carta. Informazione e formazione nei giornali locali del primo Novecento, Settegiorni, Pistoia 2012, pp. 28 e 32; Bernardino Carbonici, cenni autobiografici, «La Risveglia», settembre-dicembre 2000, dove si fa riferimento a «scaramucce» politiche e intellettuali che videro come protagonista Targioni nella Toscana degli anni Dieci.

57 Cfr. Lotta e risultati, «L'Avvenire», 2 novembre 1913. 
in altre regioni d'Italia e persino all'estero, per i lavoratori italiani emigrati in Svizzera e Francia ${ }^{58}$. A coronamento della peculiare belle époque di Targioni giunse, nell'anno della 'Settimana rossa', l'elezione a consigliere di minoranza nel Comune di Cerreto Guidi (poco distante da Empoli, Fucecchio, San Miniato e Vinci, nella Provincia di Firenze) e, soprattutto, la nomina a sindaco di Lamporecchio, all'epoca prima amministrazione socialista del circondario di Pistoia e una delle poche in Toscana. Infatti, a differenza di altre regioni - come Lombardia o Emilia-Romagna -, fino al 1920 le amministrazioni socialiste toscane sarebbero state rare e di breve durata. Si ebbero esperienze simbolicamente importanti come quelle di Colle Val d'Elsa dal 1897, di Sesto Fiorentino dal 1899, di Castelfiorentino e Piombino dal 1902, e di altri sette comuni (tra cui Massa e Prato) negli anni successivi; ma nell'anno dell'attentato di Sarajevo solo altri sette municipi, quattro dei quali nel Valdarno inferiore, furono conquistati dai socialisti, mentre altri venivano persi.

Stando a un rapporto redatto dai carabinieri per il sottoprefetto di Pistoia il 24 luglio 1914,

Gli elettori socialisti delle sezioni delle frazioni di San Baronto e Porciano, che per primi ebbero i risultati dello scrutinio [...] si riversarono nel capoluogo di Lamporecchio, percorrendo l'unica via che attraversa il paese con il loro vessillo spiegato e cantando l'inno per la vittoria riportata. [...] Terminato il comizio, un nucleo di circa cento persone in gran parte giovinastri sempre a bandiera spiegata e cantando si avviò verso il palazzo comunale e colà arrivato, inneggiando al socialismo gridarono che volevano la bandiera del Comune loro conquistato, e così venne inalberata per circa un'ora e mezza ${ }^{59}$.

Targioni «al grido di "Viva Lamporecchio socialista" ribadì che non si trattava di un successo di uomini, ma del trionfo di un'idea di fratellanza senza confini e senza tramonto» ${ }^{60}$.

La prima adunanza della nuova amministrazione comunale fu tenuta il 30 luglio e, insediata la Giunta, il neosindaco Targioni illustrò un programma di governo riformista (con progetti di intervento pubblico su viabilità e infrastrutture, scuola e assistenza sanitaria, revisione delle imposte e trasparenza nelle decisioni) e concluse sottolineando che

Il Comune fino da oggi non sarà più il monopolio di pochi, ma la casa del popolo ove tutti potranno convenire; una cooperativa di cui i contribuenti saranno gli azionisti i quali ritrarranno da quella i vantaggi sotto la forma di pubblici servizi. [...] Da que-

58 Rapporti su conferenze e comizi di Targioni a Radicondoli, Massa Marittima, Vinci, Brozzi, Firenze, Lugano, Berna, Ginevra, Lione e altrove sono conservati in ACS, CPC, b. 5032, cit.

59 Cit. in Poli, Lamporecchio, cit., pp. 66-67.

${ }^{60}$ Ivi, p. 67. 
sti banchi riaffermiamo le nostre idealità politiche verso una radicale trasformazione sociale che a base dell'umano consorzio ponga la proprietà collettiva. Ci dichiariamo solidali con tutti gli sfruttati e contro gli sfruttatori. Il nostro saluto vada alle vittime della reazione che accenna a ritornare, la nostra esecrazione alla guerra spogliatrice e brigantesca. [...] Come difendiamo i diritti del popolo da questi banchi, li difenderemo domani, se occorre, in piazza e sulle barricate ${ }^{61}$.

Il programma venne approvato dal Consiglio comunale ma, meno di un mese dopo, fu significativamente emendato in alcune parti dal prefetto di Firenze ${ }^{62}$.

Eravamo nel pieno della crisi dell'estate 1914 e a fine luglio era scoppiata la guerra europea, subito divenuta mondiale. Mentre l'Italia manteneva la sua neutralità, Targioni espresse una netta opposizione all'intervento in guerra sia in sede consiliare - il giorno dell' insediamento, come abbiamo visto, e nella seduta successiva quando, il 20 agosto, commemorò Jean Jaurès, in Parigi «da mano assassina trucidato», e fece approvare una delibera per rinominare la piazza di fronte al Municipio «Piazza Giovanni Jaurès ${ }^{63}$ (attuale piazza Francesco Berni) -, sia con una nuova opera antibellica non casualmente intitolata La conflagrazione europea. Poema antiguerresco (Tip. U. Polli, Firenze 1914).

Nell' introduzione di questo «povero libriccino» dedicato «agli operai e specialmente agli obliati lavoratori delle braccia», Targioni affermava «che la guerra tra stati e stati è guerra di conservazione fatta unicamente nell' interesse delle dinastie, del militarismo e delle classi reddituarie e non è guerra di liberazione delle classi lavoratrici dal giogo tirannico della classe capitalistica». Nel preludio in versi, poi, dava un'ulteriore descrizione della finalità della sua opera, rievocando in modo riconoscibile il proemio dell'Orlando furioso, un'opera che all'epoca circolava ampiamente nelle campagne toscane e che sappiamo venir recitata a memoria da non pochi lavoratori dei borghi e delle campagne ${ }^{64}$ :

${ }^{61}$ Archivio Storico del Comune di Lamporecchio (ASCL), Serie I.37 Consiglio 1913-1916, Adunanza 30 luglio 1914. Cfr. R.L. Aiazzi, S. Papini Piquè e L. Cecchi (a cura di), Inventario dell'Archivio del Comune di Lamporecchio, Comune di Lamporecchio, Lamporecchio 2014.

${ }^{62}$ Cfr. ASCL, Serie I.37 Consiglio 1913-1916, Adunanza 30 luglio 1914, e Intervento prefetto, 24 agosto 1914. Altre delibere sarebbero poi state emendate dalla Prefettura, come quella indirizzata al governo per chiedere l'amnistia per i condannati a seguito della 'settimana rossa', cfr. ivi, Adunanza $1^{\circ}$ settembre 1914, e Intervento prefetto, 30 dicembre 1914.

63 ASCL, Serie I.37 Consiglio 1913-1916, Adunanza 20 agosto 1914.

${ }^{64}$ Un altro esempio di adattamento del poema dell'Ariosto lo troviamo in G. Gagli, La Baccheide (Poema eroicomico in ottava rima). La rivolta contro la guerra delle donne di Greve in Chianti 1 maggio 1917, a cura di C. Baldini, Comune di Greve in Chianti, Greve in Chianti 1998; per la genesi dell'opera cfr. R. Bianchi, Donne di Greve. Primo maggio 1917 nel Chianti. Donne in rivolta contro la guerra, Odradek, Roma 2004. 
Gli empi apparati bellici di Marte,

Gli stratagemmi e i piani fraudolenti

Premeditati dal mal genio e l'arte

Della guerra, il furor dei combattenti,

L'onor, le gesta militari; intanto

Non saranno materia al nostro canto.

Ma l'orror delle stragi e delle morti,

Le grida, i pianti, le miserie, i lutti

Di milioni di madri e consorti;

Campi, borghi, cittadi arsi e distrutti,

Sottoporremo all'occhio del pensiero

Col verso umile sì ma vero.

[...]

Da questa modestissima tribuna

Insorgeremo in tutto l'odio nostro,

Contro i delitti che scatena e aduna

La guerra; iniquo e detestabil mostro,

Cui cieca invidia, lo sfrenato orgoglio

E l'ignoranza, han conservato i soglio ${ }^{65}$.

Se le opere di Targioni avrebbero poi ispirato poeti improvvisatori, come Vasco Cai ${ }^{66}$, il conflitto mondiale avrebbe assunto dimensioni e caratteri inattesi, tanto da ridefinire schieramenti, categorie politiche, punti di riferimento ideali. Anche i paesi e le campagne più sperdute sarebbero stati attraversati da nuove fratture. Dopo, con l'intervento e la guerra totale, tutto sarebbe cambiato; anche per Targioni.

\section{Il Convertito: nella guerra mondiale}

Durante le settimane del 'Maggio radioso', mentre nelle campagne e nei borghi toscani si susseguivano manifestazioni e folte proteste contro la guer$\mathrm{ra}^{67}$, e mentre nel centro di Firenze e di altre città cortei di interventisti riuscivano ad attirare l'attenzione dei principali quotidiani nazionali così come avrebbero poi calamitato l'attenzione di molti storici, Targioni fu una delle tante persone ripetutamente fermate o arrestate per il coinvolgimento nelle

65 Targioni, La conflagrazione europea, cit.

${ }^{66}$ Cfr. F. Franceschini, I contrasti in ottava rima e l'opera di Vasco Cai da Bientina, Pacini, Pisa 1983, p. 42; D. Priore, L'ottava rima, Comune di Terranova Bracciolini, Terranova Bracciolini 2002, p. 193.

${ }^{67}$ Questo, ovviamente, non avveniva dappertutto, come mostrano i saggi di Leo Goretti e Simone Lisi in R. Bianchi (a cura di), La Grande Guerra lontano dal fronte. Barberino Val d'Elsa e Tavarnelle Val di Pesa 1914-1918, Pacini, Pisa 2018. 
mobilitazioni contro l'intervento ${ }^{68}$. Tra le accuse vi era quella di aver ispirato i tumulti avvenuti a Empoli tramite le colonne del giornale socialista «Vita Nuova» ${ }^{69}$; la Sottoprefettura di Pistoia arrivò persino a chiedere lo scioglimento dell'amministrazione di Lamporecchio ${ }^{70}$. Tre giorni dopo l'intervento italiano in guerra, il prefetto di Firenze avrebbe comunicato alla Direzione generale di Pubblica sicurezza che Targioni era stato redarguito dal comandante della stazione carabinieri di Lamporecchio

per la condotta tenuta durante la presentazione dei richiamati e per diffidarlo per l'avvenire. Il Targioni, eccitandosi, pronunziò parole di oltraggio contro il Comandante e questi lo dichiarò in arresto in flagranza; perquisitolo, gli rinvenne addosso due manoscritti destinati alla stampa, in uno dei quali si contengono espressioni ispirate a critica violenta della guerra, tali da provocare agitazioni. Il Targioni sarà denunziato all'Autorità giudiziaria ${ }^{71}$.

Il comandante dei carabinieri faceva riferimento all'atteggiamento tenuto dai vertici del Comune in occasione della partenza dei richiamati della classe 1891, quando l'assessore anziano, il socialista Alfredo Vescovi - un piccolo commerciante rivenditore di vino, socialista «con tendenze centriste» e futuro sindaco nel 1920 -, partecipò a una manifestazione per la pace. Il processo contro il sindaco non avrebbe avuto «luogo in seguito amnistia», ma il nuovo periodo in carcere cadde in un momento particolare delle relazioni personali di Targioni e, forse anche per queste vicende più intime, segnò la svolta decisiva per la sua storia politica ${ }^{72}$.

Il sindaco avrebbe poi scritto che l'arresto era il risultato di una macchinazione ordita da un funzionario del Comune (in combutta con le «autorità politiche» e la «questura») con cui era entrato in contrasto per motivi riguardanti criteri d'acquisto di cancelleria e beni di consumo dell'amministrazione, ovvero per interessi che legavano il funzionario ad alcuni fornitori. Sullo sfondo di queste trame, ritenute certe da Targioni, e all'indomani delle mobi-

${ }^{68}$ Cfr. S. Soldani, La Grande Guerra lontano dal fronte, in Storia d'Italia. Le regioni, cit., pp. 426-452; R. Bianchi, Il fronte interno alla prova. Le opposizioni alla guerra a Prato e in Tosca$n a$, in D. Menozzi, G. Procacci, S. Soldani (a cura di), Un paese in guerra. La mobilitazione civile (1914-1918), Unicopli, Milano 2010, pp. 105-132; C. Poesio, Firenze, in F. Cammarano (a cura di), Abbasso la guerra! Neutralisti in piazza alla vigilia della Prima guerra mondiale, Le Monnier, Firenze 2015, pp. 447-458.

69 Cfr. Guerrini, Il movimento operaio nell'Empolese, cit., pp. 143-147, ma per uno studio più recente cfr. D. Lovito, La Grande Guerra in una piccola città, «Quaderni d'Archivio», V (5), 2015, pp. 27-51, e soprattutto Id., Empoli tra Grande Guerra e fascismo (1914-1921), Tesi di laurea magistrale, Università degli Studi di Firenze, rel. R. Bianchi, a.a. 2013/2014, pp. 26-44. 70 ASP, Sottoprefettura, f. 691, Proposta di scioglimento dell'amministrazione comunale, 16 maggio 1915.

71 ACS, CPC, b. 5032, cit., Prefetto di Firenze a DGPS, 27 maggio 1915.

72 Ivi, Prefetto di Firenze a MI, 6 giugno 1915. Su Vescovi cfr. Poli, Lamporecchio, cit., p. 73. 
litazioni contro l'intervento in guerra, una mattina il «reggente provvisorio la stazione» dei carabinieri di Lamporecchio si fece accompagnare dal sindaco in caserma. Qui Targioni «venne insultato e maltrattato nel modo peggiore e chiuso in camera di Sicurezza. [...] Rimase digiuno fino a tarda sera quando un suo vicino parente gli portò da mangiare» ${ }^{73}$.

Forse è vero che qualcuno tramò contro il sindaco per interessi personali, ma è difficile verificarlo. Sta di fatto che Targioni, socialista nonché poeta contadino e cantore antimilitarista, subì l'ondata repressiva che colpì tutta l'opposizione alla guerra ${ }^{74}$. Una repressione che riuscì a cogliere molti frutti e anche a ottenere risultati forse inattesi.

Come avrebbe scritto negli abbozzi della sua autobiografia e poi in altri testi, alcuni resi pubblici, in prigione Idalberto si sentì lasciato solo dai compagni di partito. Con questo ennesimo abbandono subito, Targioni mise in discussione il neutralismo e il pacifismo, la teoria e la pratica della lotta antimilitarista e della militanza socialista ${ }^{75}$. Era l'Internazionale che lo aveva tradito votando i crediti di guerra, rinunciando allo sciopero generale che avrebbe dovuto bloccare la macchina bellica, abbandonandolo in carcere. Verso l'idea socialista Targioni montò un desiderio di vendetta irrefrenabile:

Rimasi quale un buon uomo perdutamente innamorato di bellissima donna nelle cui virtù ripose tutta la sua fede per l'appagamento e il conforto [...] quando, all'improvviso, e nel momento che si aspettava le prove maggiori d'affetto e di buone opere, ebbe ad accorgersi che essa altro non era se non una volgare sgualdrina dominata dalle più basse passioni ${ }^{76}$.

«In tale occasione ebbe modo di riscontrare fino a qual punto giungeva l'affetto e l'interessamento dei compagni per lui e di qual genere fosse il loro coraggio e la loro fede», avrebbe scritto con risentimento in un testo reso pubblico e parlando di sé alla terza persona:

Di cento iscritti alla Sezione uno solo si presentò la notte al passaggio della carrozza che lo portava ammanettato a Pistoia. Nessuno di loro, dopo quel giorno, si recò ad offrire un aiuto, a porgere un conforto alla sua famiglia, a chiedere del suo stato di salute (era anche allora ammalato) a chi vigilava il suo carcere. Bastò quel piccolo infortunio perché tutti se la dessero a gambe come un sacco di lepri! ${ }^{77}$.

73 Targioni, Vent'anni, cit., p. 7.

74 Per un inquadramento cfr. M. Isnenghi e G. Rochat, La Grande Guerra 1914-1918, La Nuova Italia, Firenze 2000, pp. 303-347.

75 Cfr. AIT, Scatola 3.31, Cinquant'anni, cit. p. nn. Su questo passaggio Targioni sarebbe tornato più volte. Cfr. ad esempio i testi in Archivio di Stato di Pistoia (ASP), Sottoprefettura, b. 60, f. 744 1918-1921 Amministrazioni comunali. Affari diversi, dove è anche conservata una copia del suo libretto Vent'anni, cit.

76 AIT, Scatola 3.31, Cinquant'anni, cit. p. 3.

77 Targioni, Vent'anni, cit., pp. 7-8. 
La delusione verso l'Internazionale socialista, «così bene organizzata contro la guerra che in Germania i socialisti furono della guerra i più ardenti sostenitori», generò un odio implacabile verso i compagni di partito. Annunciò di non poter più seguire le direttive del Psi e di volersi ritirare a vita privata. Intanto, la Prefettura di Firenze scriveva alla Sottoprefettura di Pistoia per chiedere di non procedere con lo scioglimento di Lamporecchio e il sindaco veniva rilasciato ${ }^{78}$.

Diversi mesi dopo, nel 1916, Targioni rese noti i dubbi che lo attanagliavano in una lettera inviata - a suo dire - all'«Avanti! », che però non sarebbe stata pubblicata dal quotidiano socialista. L'elaborazione del testo doveva essere stata faticosa anche perché, nel pieno della tormenta politica, Sofia Bacarelli era di nuovo incinta (di Idalberta) e Targioni, sempre in difficoltà economiche, era alla ricerca di un lavoro. Non esitò a chiedere una qualche forma di 'spinta' a «un ministro» e a «un marchese», come scrisse confidenzialmente il 21 aprile 1916 a Domizio Torrigiani - amico fedele «per lungo svolgere di anni» -, dicendosi fiducioso in «un valido appoggio in un affare che, se riuscirà, potrò dare un calcio alla miseria, ma pensando a quanto sono stato disgraziato temo che il diavolo ci metterà la coda! Come vedi attraverso un periodo di forte trepidazione». In quella lunga missiva, Targioni si diceva prossimo a scrivere la lettera per il quotidiano socialista, argomentò dubbi e prese di posizione:

In quanto al partito sono così risoluto di andarmene che ho già preparato il mio congedo intitolato 'Perché me ne vado' e domani lo manderò all'Avanti!

È un articolo che avrà - se non altro - il pregio della lealtà e della franchezza dove ho detto chiaro e tondo il motivo per cui ho deciso di abbandonare il partito.

Siccome io sono troppo conosciuto e non posso andarmene alla chetichella, darei luogo a troppe malignazioni. Invece mettendo le carte in tavola o dovranno lasciarmi in pace o affrontare la riflessione la quale, stai certo, non sarebbe di loro gusto e convenienza! Forse preferiranno il modo più spiccio, quello di consegnare il mio scritto al cestino, ma in questo caso ricorrerò all'ospitalità di altri giornali. Presto t'informerò della faccenda!

Apprendo con piacere tutto l'intelligente lavoro che vai compiendo costà [...] ti seguo col pensiero e ogni giorno che passa più sono preso dal desiderio che la guerra termini presto e colla fortuna delle nostre armi. Anzi, a questo punto dico: guai se non si avesse a vincere! Ma ti confesso sinceramente che non sono ancora riuscito a capire perché gli uomini debbano uccidersi per intendersi e perché l'Italia non avrebbe potuto evitare una guerra così disastrosa come l'hanno evitata tanti altri stati europei. Comunque, a diritto od a torto, oramai la nave è partita mentre il mare era in grande tempesta. Forse era meglio restare bene ancorati nel porto nostro, ma poiché questo non è avvenuto chi è nato in Italia ha il dovere di pensare o di dire: «tutta la nave è piena di nostri fratelli,

78 Ivi, p. 10; cfr. ASP, Sottoprefettura, f. 691, Risposta del Prefetto di Firenze al Sottoprefetto di Pistoia, 31 maggio 1915, cit. in Poli, Lamporecchio, cit., p. 70. 
in essa si raccolgono i tesori della nostra civiltà, che è la prima del mondo, chi di voi dunque potrà desiderare che la nave affondi? Chi non darà ciò che può perché questa nave torni vittoriosa alle rive della patria?» Chi pensa diversamente, per me, se non è un imbecille, è un pazzo birbante ${ }^{79}$.

Ma le domande e le incertezze, confessate in privato all'amico massone e volontario di guerra, non potevano trovare spazio nell'Italia della mobilitazione totale, delle trincee interne, dei lutti. Nel passaggio delle frasi dalla dimensione privata a quella pubblica, uscivano dal setaccio solo gli slogan interventisti e le argomentazioni patriottiche ${ }^{80}$, mentre anche a Lamporecchio si dovevano contare le vittime per un tributo di sangue che in quelle terre era pagato da «coloni, pigionali, fabbri, calzolai e falegnami», stando ai dati sui militari caduti al fronte raccolti dalla Sottoprefettura di Pistoia ${ }^{81}$.

Oramai Targioni - in difficoltà economica, con due famiglie e vari figli da mantenere - si era spostato irrimediabilmente su posizioni belliciste, forse anche sperando che l'onda montante dell' interventismo totale avrebbe potuto portare qualche vantaggio agli accoliti della causa nazionale. Gli accenti della sua mobilitazione bellica intrecciavano temi cari all'interventismo 'democratico' e a quello 'rivoluzionario', come emerge dalla lettera che sarebbe stata cestinata dall' «Avanti!» e che fu invece pubblicata con un certo rilievo dal quotidiano fiorentino «Il Nuovo Giornale», il 17 maggio 1916 a pagina 2, poi ripresa anche da un altro «giornale borghese», secondo le parole di Targioni, come «Il Popolo pistoiese» - che si chiese se quello del sindaco ex socialista fosse «un ravvedimento, una resipiscenza» oppure «una conversione»-, e dal settimanale repubblicano cesenate «Il Popolano». Nel testo, si scagliava contro «l'arrivismo politico di tanti compagni», denunciava l'abisso «fra socialismo e sedicenti socialisti , lanciava «un monito» ai «compagni» e «a tutti gli operai»:

Educatevi! Istruitevi! Imparate a conoscere il mondo, ciò̀ gli uomini e le cose, ma soprattutto gli uomini [...], fintanto che seguirete ciecamente degl'individui che non sono scaturiti dalle viscere della vostra classe e non hanno dato prove sufficienti di

79 ISRT, ADT, Serie I Carteggio, I.20.1, Targioni a Torrigiani, 21 aprile 1916; sottolineato nel testo. Cfr. AIT, Scatola 2.7.53, Lettera di Targioni al «Marchese», Lamporecchio 3 febbraio 1916, dove l'a. ringrazia il destinatario per avergli procurato un lavoro (venditore di calzature) che gli avrebbe permesso di vivere con più tranquillità. Non è indicato il nome del marchese, ma l'unico «marchese» nominato nell'AIT è il fascista fiorentino Dino Perrone Compagni, che marchese non era: cfr. il profilo biografico curato da Palla, in Dizionario Biografico degli Italiani, cit., vol. 82, 2015.

${ }^{80}$ Sul tema, può essere utile rileggere i testi pubblici e la corrispondenza privata di P. Calamandrei, Zona di guerra. Lettere, scritti e discorsi (1915-1924), a cura di S. Calamandrei e A. Casellato, Laterza, Roma-Bari 2006; cfr. R. Bianchi, La guerra di Piero. Calamandrei tra Grande guerra e fascismo, «Antologia Vieusseux», 40, 2008, pp. 201-205.

81 ASP, Sottoprefettura, b. 52, f. 716 1917-1918 Guerra italo-austriaca. Disposizioni per l'ordine interno, Comune di Lamporecchio a Sottoprefetto, 8 ottobre 1917. 
rettitudine, di sacrifico e di capacità, ogni vostro lavoro sarà fatalmente condannato all'insuccesso e voi proverete le più amare disillusioni.

Proletarizzate il partito e guardatevi dagli intellettuali! La decadenza del cristianesimo, come movimento di oppressi contro gli oppressori, cominciò dal giorno in cui esso accolse nel suo seno gl'intellettuali convertiti alla nuova fede. Questi, in breve tempo, riuscirono a trasformarlo in un formidabile strumento d'oppressione morale, materiale e politica qual è appunto la Chiesa Romana dei nostri giorni. Il socialismo, se non sarà preso rimedio a tempo, subirà la medesima sorte e ritarderà di tanto il suo inevitabile trionfo.

[...] malgrado il fallito internazionalismo e l'invincibile mia avversione alla guerra, con cuore di socialista italiano inneggio alla pace, ma anche alla vittoria dei nostri fratelli, soldati gloriosi di redenzione da quell'Austria che, ricordatelo compagni, noi purtroppo soltanto a parole sempre combattemmo ${ }^{82}$.

Al contempo, Targioni presentò le dimissioni dalle cariche di consigliere comunale a Cerreto Guidi e di sindaco a Lamporecchio, lamentandosi perché i socialisti della sezione lo additavano come «un convertito» e facevano circolare voci «ad arte» secondo le quali si sarebbe «compromesso con qualche alto personaggio che avrebbe promesso un impiego»: «nulla è stato concluso ancora, ma se ciò avvenisse si tratterebbe di una qualunque forma di lavoro che non vincola per nulla la mia libertà di pensiero», dichiarò in Consiglio comunale $^{83}$. Anni dopo Targioni avrebbe sostenuto che mantenne la carica di primo cittadino perché «pregato a rimanere», ma in realtà le dimissioni del sindaco furono respinte dal Consiglio con cinque voti contrari, tre a favore e una scheda bianca ${ }^{84}$.

L'Italia, dunque, era nel pieno della guerra. Lo stato d'eccezione e le leggi speciali imperavano sulla società mobilitata; si susseguivano le offensive sull'Isonzo ordinate dallo Stato maggiore guidato da Luigi Cadorna - la quarta si concluse nel dicembre 1915 e la nona 'spallata' fu nel novembre 1916 -, e intanto Targioni tendeva progressivamente a incanalarsi nel solco segnato da Mussolini a favore della mobilitazione totale e della lotta contro ogni forma di pacifismo e neutralismo ${ }^{85}$.

Targioni, insomma, fu uno dei tanti socialisti, democratici, anarchici, sindacalisti e rivoluzionari a essere sedotti dal conflitto mondiale. Per il Partito

82 Il ritiro di Idalberto Targioni dal partito socialista, e I. Targioni, Perché me ne vado, «Il Nuovo Giornale», 17 maggio 1916, corsivi nel testo; Dimissioni clamorose, «Il Popolo pistoiese», 20 maggio 1916; Idalberto Targioni, «Il Popolano», 26 maggio 1915 [sic, ma 1916, come si evince dal contenuto degli articoli].

83 ASCL, Serie I.39 Protocollo del Consiglio 1916-1918, Adunanza $1^{\circ}$ giugno 1916.

${ }^{84}$ Cfr. ibidem; per la citazione: Targioni, Vent'anni, cit., p. 11.

${ }^{85}$ La bibliografia su questa fase è sterminata. Per un orientamento si veda N. Labanca (a cura di), Dizionario storico della Prima guerra mondiale, Laterza, Roma-Bari 2014. 
socialista italiano - quello del «né aderire, né sabotare ${ }^{86}-$, questo fenomeno comportò una emorragia difficilmente sanabile, perché lo privava di buona parte del suo corpo intermedio, ovvero dei suoi intellettuali più capaci a scrivere e meglio avvezzi a parlare, verso i quali si riversò il rancore della base del partito, come ad esempio si nota leggendo una delle tante dichiarazioni che accompagnavano le donazioni all'«Avanti!»: «Piombino: Chiavacci Egidio rinnovando l'abbonamento saluta i compagni di Lamporecchio per la serietà dimostrata di fronte al voltagabbana Targioni ${ }^{87}$.

Mussolini fu il più famoso ed è ancora il più studiato per il ruolo che aveva svolto nel partito prima della guerra e soprattutto per la successiva storia sua e d'Italia. Ma persone come Targioni rappresentano in modo più efficace il dissanguamento del corpo intermedio del partito. Lo dimostrano i passaggi all'interventismo di uomini come il deputato socialista Carlo Corsi (eletto nell'ottobre 1913 al Collegio Firenze IV, espulso dalla direzione del partito nell'ottobre 1914, passato poi al fascismo nel 1920), l'avvocato livornese Michele Terzaghi (fino al maggio 1915 direttore della «Difesa», il periodico della Federazione provinciale socialista fiorentina; massone di Piazza del Gesù, futuro esponente di spicco del primo fascismo e poi del dissidentismo fascista toscano) o il ferroviere pistoiese Amulio Cipulat (già direttore dell'«Avvenire»), o come i fiorentini Ugo Forlani (presidente della Società di mutuo soccorso di Rifredi, cacciato dalla direzione della SMS alla fine del 1916 per le sue posizioni interventiste) e Alfredo Frilli (direttore didattico; poi, nel 1921, direttore del giornale fascista aretino «Giovinezza»), per ricordare solo alcuni esempi significativi ${ }^{88}$.

Il fenomeno non è stato ignorato in sede storica; già molti anni fa lo sottolineò Ernesto Ragionieri, ma si sono talvolta sottovalutati la sua estensione e il suo impatto sulla base del movimento socialista e sindacale ${ }^{89}$. In realtà la guerra spaccò tutto e comportò una trasformazione importante per il Psi, modificando i tratti del suo corpo intermedio che, nel 1919 del massimalismo,

${ }^{86}$ Cfr. G. Scirocco, Il neutralismo socialista, in Abbasso la guerra, cit., pp. 41-55.

87 Pro Avanti! Sottoscrizione 1916, «Avanti!», 15 giugno 1916; la sottoscrizione fu di 0,45 lire.

${ }^{88}$ Cfr. L. Tomassini, Carlo Corsi, in Il movimento operaio italiano, cit., vol. 2, pp. 99-100; Id., Terzaghi Michele, in Il movimento operaio italiano, cit., vol. 5, pp. 51-55; M. Terzaghi, Fascismo e Massoneria, Editrice Storica, Milano 1950, p. 10. Su Forlani a Rifredi, nel 1909 registrato alla loggia Lucifero con matricola 28701, cfr. L. Tomassini, Associazionismo operaio a Firenze fra' 800 e'900. La Società di mutuo soccorso di Rifredi (1883-1922), Olschki, Firenze 1984, pp. 299-300. Su Cipulat cfr. Petracchi, Storia di Pistoia, cit., p. 407; Morelli, Tomassini, Socialismo, cit., p. 61.

89 «Non mancavano tra i promotori del fascismo in Toscana i transfughi del movimento socialista [...]. Ma [...] si trattò quasi sempre di casi isolati e [...] privi di un forte ascendente sulle masse»: E. Ragionieri, Ilpartito fascista (appunti su una ricerca), in La Toscana nel regime fascista (1922-1939), vol. 1, Olschki, Firenze 1971, pp. 72-73. 
avrebbe avuto caratteri sociali e culturali differenti da quelli del 1914; più in generale, però, le lacerazioni sarebbero diventate insanabili e non segnarono solo il mondo socialista, ma tutte le realtà politiche e associative della sinistra e del sovversivismo, come mostra la storia del ribellismo sindacalista rivoluzionario emiliano o la biografia della femminista e anarchica Maria Rygier, per richiamare in modo sommario pochi esempi ${ }^{90}$.

Come è stato scritto negli anni del centenario, quella italiana fu una «guerra di convertiti», tra «mobilitazioni, liquidazioni e abiure» ${ }^{91}$, ed è proprio in una delle carte recentemente rinvenute nell'A rchivio Idalberto Targioni che troviamo espresse in modo più netto le ragioni dell' improvviso cambio di rotta del sindaco di Lamporecchio, ricostruite nel luglio 1920:

Fui avversario dichiarato della guerra e feci quanto era in mio potere onde scongiurarla, ma quando la guerra fu mi rifiutai di associarmi a coloro che mentre nulla avevano fatto per impedirla tentavano di fiaccare ogni spirito di resistenza allora che la guerra si era resa inevitabile e non era più possibile scongiurarla. L'essere io socialista militante non mi poteva impedire di sentirmi italiano e di desiderare e cooperare perché all'Italia arrivasse la vittoria delle nostre armi. Un siffatto atteggiamento era già stato condannato dal Partito S. U. [socialista ufficiale] anche nei riguardi di tanti altri buoni ed ottimi compagni che si erano ritirati dal Partito stesso, perciò anch'io mi decisi ad uscire dando insieme le dimissioni dalla carica di sindaco non senza rendere tutto quanto di pubblica ragione mediante una lettera aperta pubblicata sul «Nuovo Giornale» di Firenze. Quest'atto di lealtà e d'onestà politica dispiacque ai capoccioni del mio partito ed attirò sul mio capo tutti i loro fulmini. Il gregge tesserato, questa volta, abboccò all'amo. Mi considerò un traditore della sua causa e mi fece segno del suo cieco odio e insensato disprezzo. Esso, purtroppo, non comprese (e forse non poteva comprenderlo) che io era un ribelle alla dittatura di un manipolo di borghesi e di folli che si spacciavano per socialisti e che me ne era andato perché nauseato e indignato dalla bassa speculazione che si andava da essi esercitando sulla dabbenaggine e sulla credulità delle masse martoriate dalla guerra. Ciononostante seguitai, con dimessa indifferenza, a battere la mia strada certo che il tempo mi avrebbe reso giustizia. Questo giorno è giunto anche prima ch'io non lo avesse aspettato. Oggi il Partito Socialista raccoglie i frutti dei mali semi gettati: che sono quelli di avere accolto nel suo seno tanti demagoghi antiproletari quali solo miravano che ad arrivare, ed ora che sono arrivati altra preoccupazione non hanno oltre quella di conservarsi la posizione conquistata ${ }^{92}$.

${ }^{90}$ Cfr. F. Cammarano, Dalla preghiera al tumulto: un'eccedenza alla ricerca della politica, e M. Manfredi, Il neutralismo anarchico, in Abbasso la guerra, cit., pp. 1-16 e 57-69; B. Montesi, Un'«anarchica monarchica». Vita di Maria Rygier (1885-1953), Esi, Napoli 2013, cap. 3; M. Becchetti, Fuochi oltre il ponte. Rivolte e conflitti sociali a Parma (1868-1915), Derive Approdi, Roma 2013, pp. 254 sgg.

${ }_{91}$ M. Isnenghi, Convertirsi alla guerra. Liquidazioni, mobilitazioni e abiure nell'Italia tra il 1914 e il 1918, Donzelli, Roma 2015, p. 9.

92 AIT, Scatola 2.7.61, Bozza di lettera di Targioni al deputato Colajanni, Firenze, luglio 1920. 
La rottura fu profonda e, dopo Caporetto, divenne irreversibile, mentre continuava a crescere la passione verso Mussolini di Targioni, che si sarebbe dimesso dalla carica di sindaco solo nel luglio 1919.

\section{Il Traditore: fascista della prima ora}

Nell'immediato dopoguerra, in un paese in fibrillazione per il 'risveglio dei contadini' e 'della plebe', tanto auspicato anni prima da Idalberto, il «convertito» Targioni aderì al neonato movimento fascista e rese pubblica la sua presa di posizione a favore dei Fasci di Combattimento il 29 marzo 1919, subito dopo l'adunata di San Sepolcro a Milano ${ }^{93}$. Nemmeno un mese dopo, la sera di Pasqua 20 aprile - cinque giorni dopo l'assalto alla sede milanese dell'«Avanti!», distrutta da fascisti e nazionalisti -, esplose lo scontro con i socialisti di Lamporecchio e dintorni, che col ritorno dei reduci dal fronte avevano infoltito i loro ranghi; si stava dischiudendo il cosiddetto 'biennio rosso' e agli occhi di Targioni il paese si era riempito di «facce arcigne, sguardi torvi ed obliqui da ogni parte» e fioccavano «gli epiteti di venduto e rinnegato ${ }^{94}$.

Per quella sera il sindaco aveva promosso un incontro al Teatro Berni per illustrare l'attività svolta in tempo di guerra, ma una «folla di circa 400 persone giunse a Lamporecchio cantando e schiamazzando e prendendo d'assalto il teatro». Dal racconto di Targioni, sembra che i contestatori provenissero da paesi dei dintorni: «una turba raccogliticcia di fanatici» organizzata da alcuni socialisti di Lamporecchio, «tre o quattro capoccioni del luogo». La folla di «operai e quasi tutti nostri amici personali che avevamo amato come figli e strenuamente difeso, che per tanti anni educammo alla fraternità, al rispetto, alla tolleranza» - narra Targioni -, con «ogni genere d'insulti» e le «più ignominiose trivialità», minacciò e impedì di parlare ${ }^{95}$.

Nell'infuocata crisi del dopoguerra, anche a Lamporecchio si rinnovava lo scontro tra interventisti e neutralisti; la Grande Guerra aveva approfondito le fratture sociali e ridefinito le forme e i linguaggi dello scontro politico, mentre i Fasci di Combattimento già si armavano e praticavano azioni volte

93 Si veda l'articolo di Targioni in «Il Popolo pistoiese», 29 marzo 1919. Cfr. anche Bartolini, La mezzadria, cit., p. 100; Id., Una passione, cit., p. 27.

${ }^{4}$ Targioni, Vent'anni, cit., p. 18. Sulla Toscana di quel periodo rinvio a R. Bianchi, BocciBocci. I tumulti annonari nella Toscana del 1919, Olschki, Firenze 2001.

${ }_{95}$ Targioni, Vent'anni, cit., p. 19. Cfr. AIT, Scatola 3.23.47, Ill.mo Sig. Professor Bonfatti Commissario Straord. Dei Sindacati Fascisti di Firenze dedico a Lei questi rapidi cenni biografici [...] sulla mia opera socialista e di Fascista. 
all'annientamento dei nemici politici con modalità da 'guerra civile', combattuta da una parte sola ${ }^{96}$.

Nel clima da resa dei conti che segnò il dopoguerra, e nel contesto della riconversione dalla guerra alla pace, la permanenza di Targioni nella carica di primo cittadino era sempre meno giustificabile. Portato al successo dall'elettorato socialista nel 1914, il sindaco divenuto fascista fu però messo in minoranza dal Consiglio comunale solo il 14 giugno 1919. Quel giorno, finalmente, la maggioranza dei consiglieri presentò una lettera di dimissioni dalla carica; nonostante le minacciose e insistite richieste di ritiro delle lettere avanzate dalla presidenza del Consiglio comunale, tenuta da Targioni («ricordo che per decreto luogotenenziale è stata sancita la responsabilità civile per i consiglieri che durante lo stato di guerra provocheranno, senza giustificati motivi, lo scioglimento delle amministrazioni comunali» e la «responsabilità specialmente nei riguardi della disoccupazione operaia...»), l'intervento del socialista Vescovi ruppe gli indugi e il consiglio votò a scrutinio segreto. «La votazione che à luogo a palle bianche e nere e che viene effettuata separatamente per ciascuno dei consiglieri dimissionari dà i seguenti risultati»: 7 voti a favore contro 3 per i consiglieri Dario Meoni, Cesare Meozzi e Giulio Torrigiani, 6 a 3 per Pietro Biondi, Eliseo Cei ed Ezio Fanti, e 5 a 4 per Alfredo Vescovi ${ }^{97}$. Il Comune venne commissariato e la prima riunione della Giunta diretta dal commissario prefettizio Umberto Petragnani si svolse il 9 luglio, quella del Consiglio il 28 agosto 1919 col nuovo commissario Euclide Zolli ${ }^{98}$.

Come moltissime amministrazioni comunali elette alla vigilia della guerra, dunque, anche quella di Targioni si sciolse prima della fine del mandato. Non è stato fatto un censimento dei comuni commissariati tra guerra e dopoguerra in Italia, ma sappiamo che furono numerosi e che spesso vennero travolti da denunce e processi esplosi per scandali riguardanti la gestione delle finanze e della politica annonaria, come nel caso dello 'scandalo delle stoffe' a Firenze. Anche sul Comune di Lamporecchio si addensò qualche ombra, ma non emerse niente di significativo. Sta di fatto, però, che il commissario prefettizio si insediò proprio nei giorni dei moti contro il caroviveri, che nell'estate 1919 attraversarono tutta Italia e che in Toscana vennero ricordati come il 'Bocci-Bocci' '99.

96 Cfr. F. Fabbri, Le origini della guerra civile. L'Italia dalla Grande Guerra al fascismo, 1918 1921, Utet, Torino 2009; R. Bianchi, Pace, pane, terra. Il 1919 in Italia, Odradek, Roma 2006. 97 ASCL, Serie I.40, Consiglio 1918-1922, Adunanza 14 giugno 1919. La seduta, iniziata alle 16, terminò alle 20.30 .

98 Cfr. ASCL, Serie I.40, Consiglio 1918-1922, Adunanza 28 agosto 1919; ivi, Serie I.41 Giunta 1918-1921, Adunanza 9 luglio 1919. Anche Zolli fu poi sostituto a ottobre e un nuovo commissario si insediò a dicembre, cfr. Poli, Lamporecchio, cit., p. 73.

99 Cfr. Bianchi, Bocci-Bocci, cit., dove non sono segnalati tumulti a Lamporecchio. 
Alle elezioni politiche di novembre i socialisti di Lamporecchio ottennero il 63\% dei voti e con le amministrative dell'autunno 1920 riconquistarono il comune con una schiacciante maggioranza che portò Alfredo Vescovi alla guida di una giunta che però si sarebbe dissolta pochi mesi dopo, sotto l'offensiva congiunta di squadrismo e apparati dello Stato ${ }^{100}$.

Targioni non si era candidato, ma continuava ad essere ben presente sulla scena politica, indirizzando i suoi attacchi contro i compagni di un tempo, $\mathrm{i}$ «socialisti disciplinati» che ora agivano «secondo gli ordini emanati da Mosca», «piccoli demagoghi capaci solo a fare un atto di violenza quando si trovano in cento contr'uno»; ad essi, nel 1920, Targioni porgeva il suo ironico e minaccioso saluto: «Bravi ragazzi, così va bene. Ci riparleremo fra qualche tempo. Intanto salute e arrivederci sulle barricate ${ }^{101}$.

Usando forme di comunicazione e linguaggi consolidati ma rinnovati per l'occasione, Targioni avrebbe scavato un solco che contribuì a instradare il fenomeno più ampio e contraddittorio del consenso di una parte del mondo contadino al fascismo ${ }^{102}$. Concorse alla costituzione di sezioni fasciste nell'area (come a Larciano), divenne Segretario del Fascio di Combattimento di Lamporecchio e in breve tempo si affermò come uno dei più influenti elementi in camicia nera delle zone agrarie del Pistoiese, svolgendo un ruolo significativo nel fascismo di tutta la provincia fiorentina dove intanto, nel dicembre 1920, gli squadristi avevano ucciso un vecchio mezzadro mugellano, prima vittima di una lunga serie $e^{103}$.

La 'battaglia di Firenze' iniziata il 27 febbraio 1921 e i 'fatti di Empoli' del primo marzo coinvolsero anche Lamporecchio, dove per arrestare il transito dei fascisti si mobilitò «la popolazione intera armata di fucili, roncole e coltelli comprese molte donne che costruirono barricate nelle strade ${ }^{104}$. Il sindaco socialista Vescovi, ritenuto «il diretto organizzatore dei disordini» fu denunciato per associazione per delinquere e si trovò costretto alle dimissioni dopo poche settimane, il 20 aprile. Mentre lo squadrismo e i sostenitori dei blocchi 'dell'ordine' si impadronivano della regione, il governo locale restò in agonia per un certo periodo e passò infine sotto il controllo di un commissario prefettizio lieto di sottolineare che il Municipio «cedesse le armi così come i negatori della Patria le cedevano, per innata vigliaccheria, dinanzi al

\footnotetext{
100 Cfr. Poli, Lamporecchio, cit., p. 73.

101 Targioni, Vent'anni, cit., p. 31, corsivo nel testo.

102 Cfr. A. Ricci, Detto e taciuto. Le ottave del consenso contadino al regime fascista, «La Ricerca Folklorica», 11, 1985, pp. 121-124.

103 Cfr. Petracchi, La genesi, cit., p. 103; M. Palla, Firenze nel regime fascista (1929-1934), Olschki, Firenze 1978, p. 128; E. Ronconi, Antifascismo borghese 1922-1930, «Italia contemporanea», 1980, 140, p. 20.

104 Poli, Lamporecchio, cit., p. 75. Sui 'fatti' cfr. P. Pezzino, Empoli antifascista. I fatti del $1^{\circ}$ marzo 1921, la clandestinità e la Resistenza, Pacini, Firenze 2007.
} 
nemico ${ }^{105}$. La rotta di Caporetto distava tre anni mezzo, ma era ben viva e presente nella retorica delle autorità.

L'apparato statale e il giovane fascismo frantumavano le ultime roccaforti del socialismo toscano e intanto Targioni partecipava direttamente alle azioni squadriste (come, da quel che sembra, in occasione della devastazione della sede comunista di Vinci nel giugno 1921), sosteneva l'opposizione al 'patto di pacificazione' in agosto (dopo i 'fatti' di Sarzana) e diveniva un ideologo (forse il più importante in Toscana) della reazione fascista contro le rivendicazioni contadine e mezzadrili ${ }^{106}$. Difficile dire, allo stato attuale della ricerca, quale sia stato il ruolo esatto di Targioni nel movimento squadrista. Il dato dovrà essere verificato con più attenzione tra le carte del suo archivio. Sembra però certo che, piegando e adattando aspetti della propaganda fascista alle peculiari caratteristiche della regione, e talvolta entrando anche in polemica con dirigenti fascisti della pianura padana, Targioni trasformò la posizione «populista» del movimento nazionale in una dottrina sostanzialmente autoritaria e antimezzadrile, espressa anche nell'articolo Sindacalismo e fascismo dove pure lamentava il mancato rispetto del patto colonico da parte degli agrari e la mancata assunzione di braccianti agricoli disoccupati ${ }^{107}$. I mezzadri erano presentati, dall'ex socialista, come partner dei padroni secondo una logica di compartecipazione, nella quale l'agrario aveva il dovere di elevare dal punto di vista morale il contadino mentre il colono doveva investire tutte le energie familiari nel lavoro per il podere, in nome di una produttività generale che riguardava l'impresa comune, compartecipata. I mezzadri, considerati dei «rapaci», non avevano alcuna legittimazione, nessun motivo per rivendicare trasformazioni di tipo sociale o modifiche sostanziali dei patti colonici; mentre invece risultava umanamente comprensibile, agli occhi di Targioni, che gli agrari potessero usare tutti i mezzi a loro disposizione per cacciare dai poderi le famiglie mezzadrili qualora fossero divenute nemiche dell'impresa compartecipata $^{108}$. I Fasci di Combattimento non si erano ancora trasformati in Partito nazionale fascista (Pnf) quando le camicie nere rivolgevano ai contadini minacce sotto forma di consigli. Lo dimostrò, ad esempio, Targioni provando a spiegare ai mezzadri che i lavoratori salariati avrebbero potuto muoversi contro di loro in qualsiasi momento; pigionali, braccianti e altri operai agricoli costituivano «un esercito di riserva» pronto a spodestare le famiglie mezzadrili dalla conduzione dei poderi. Il consiglio/minaccia non rimase lettera morta,

\footnotetext{
105 Relazione del Commissario prefettizio Antonio Lucchesi, cit. ivi, p. 75.

106 Cfr. Poli, Lamporecchio, cit., p. 77.

107 Sindacalismo e fascismo, «La Nazione», 8 luglio 1923; F. Snowden, The Fascist Revolution, cit., p. 83. Cfr. R. Cantagalli, Storia del fascismo fiorentino 1919/25, Vallecchi, Firenze 1972, pp. 354-355.

108 I. Targioni, I doveri del contadino, «La Riscossa», 3 giugno 1922; F. Snowden, The Fascist Revolution, cit., pp. 83 sgg., per la cit.: ivi, p. 89.
} 
se è vero che nell'agosto 1921 in provincia di Firenze si contarono 300 sfratti di famiglie mezzadrili, gettate nella miseria e sostituite da contadini legati alle varie sezioni fasciste ${ }^{109}$.

In quei mesi Targioni, sempre in contatto col possidente Dino Philipson, fece un giro di propaganda nei paesi che tante volte avevano udito le poesie e i canti del 'Diavolo rosso':

Dopo tanti anni abbiamo potuto riudire anche qui la parola del caro Targioni. Tutti in Montelupo ricordano le sue calde improvvisazioni poetiche e le sue conferenze socialiste. Ma questa volta il Targioni tornava fra noi per parlare non del socialismo ma dei Fasci di Combattimento. Perché questa strana conversione?

Per Targioni «il socialismo possibile e attuabile» era ormai «tutto compreso nei Fasci di Combattimento ${ }^{110}$. Egli non aveva rinnegato il socialismo, ma era «l'indirizzo politico e pratico dei socialisti» a trovarsi «fuori di quella direttiva in altri tempi seguita»; e poi - scriveva - «mi hanno preceduto socialisti come Berenini, Cabrini, Badaloni, Bissolati [...]. Ma anche se avessi io radicalmente cambiato le mie idee, cosa ci sarebbe per questo da dire? E quanti non hanno cambiato anche fra gli uomini più sommi?» ${ }^{111}$.

Facendo leva sulla collaudata esperienza pubblicista, collaborò con numerosi periodici fascisti, tra cui «Giovinezza» (1921-1923), «La Riscossa» (1921-1922), «L'Azione fascista» (1922-1923), «Battaglia fascista» (19241926), e ne fondò due: «L'Alleanza» (1921) e «L'Ordine» (1922). Nella primavera 1921 il Fascio di Lamporecchio contava già 30 iscritti (a Pistoia erano 350 , poco più di 6.350 in Toscana) e un anno dopo aveva triplicato il numero di aderenti ${ }^{112}$. Fu proprio sulle pagine della «Riscossa» che nell'aprile 1922 l'ex poeta-contadino arrivò a sostenere che «la classe dei contadini gode oggi uno stato di relativa agiatezza e i miliardi depositati negli istituti di credito dai contadini, quelle migliaia di loro che si son comprati il podere, il lusso e le comodità di cui essi fanno largo sfarzo sono elementi sufficienti a provarlo» ${ }^{113}$.

Dopo la marcia su Roma e la formazione del primo governo Mussolini, nel 1923 Targioni venne nominato Segretario dei Sindacati Fascisti per l'Agricol-

109 Cfr. Snowden, The Fascist Revolution, cit., p. 90.

110 «L'Alleanza», 21 aprile 1921, cit. in Poli, Lamporecchio, cit., p. 77. Su Philipson cfr. le ricorrenze nell'Elenco di consistenza; liberale, massone e figura chiave del primo fascismo pistoiese, Philipson cadde in disgrazia nel 1925 con la repressione della libera muratoria e fu confinato a Eboli; nel 1944 venne nominato sottosegretario dal governo Badoglio. A Eboli, dal 2004 una targa lo celebra come antifascista. Cfr. Bianchi, Massoneria e fascismo, cit., pp. $118,137$.

111 I. Targioni, La parola all' imputato, «L'Alleanza», 4 marzo 1921.

112 Per questi dati, relativi a maggio 1921 e maggio 1922, cfr. Petracchi, La genesi, cit., pp. 98-99.

113 «a Riscossa», 18 aprile 1922, cit. in Cantagalli, Storia del fascismo, cit., p. 354. 
tura della Provincia di Firenze, a riconoscimento dell'opera di propaganda e di persuasione svolta presso le masse contadine, e nel 1924 fu eletto consigliere provinciale, come attesta anche il suo biglietto da visita conservato all'Istituto degli Innocenti ${ }^{114}$.

Ma la frattura con una parte importante della società locale, e non solo con gli ex compagni di Lamporecchio, non era più ricomponibile, in una Toscana marcata a ferro e a fuoco dalla Grande Guerra, dallo squadrismo e dalla marcia su Roma. Molti anni dopo Lida Chiappini, una delle nipoti di Targioni, figlia di Velia, avrebbe raccontato che «Un giorno durante un comizio in piazza Berni [di fronte al Municipio di Lamporecchio] la gente, in segno di protesta, indossò la giubba rivoltata ed in molti, dal teatrino adiacente, gli buttavano delle monetine per umiliarlo» ${ }^{115}$.

\section{Il Pentito: verso l'oblio}

Dopo il delitto Matteotti, negli anni di costruzione del regime, quando persino il vecchio amico Domizio Torrigiani - che col Grande Oriente d'Italia aveva sostenuto l'interventismo e poi il primo fascismo - subiva la persecuzione mussoliniana e veniva preso di mira dalla 'seconda ondata' squadrista che nell'ottobre 1925 incendiò la sua villa a San Baronto, Targioni abbandonò l'attività poetica e smise di pubblicare libri o raccolte di testi ${ }^{116}$.

Tornò a farlo nei suoi ultimi suoi mesi di vita con un Calendario agricolo fascista scritto nel 1929 e pubblicato l'anno dopo. L'opera si apriva con un saluto al «camerata» Targioni redatto da Scipione Picchi - figlio di un possidente agrario, fondatore del Fascio di Greve, poi dirigente del partito ${ }^{117}-\mathrm{e}$ con la prefazione del meno conosciuto prof. Cesare Balsimelli, intenzionato a rendere giustizia alla storia di vita del fascista Targioni:

114 Su queste vicende si trovano molti riferimenti nell'AIT soprattutto per quello che riguarda l'attività di segretario del sindacato agricolo, meno per quella di consigliere provinciale. Per il biglietto da visita cfr. AOIF, Balie e Bambini 1868, cit.

115 Cit. in Poli, Lamporecchio, cit., p. 80. La notizia fu raccolta nel 1996, ma non si conosce la data dell'episodio narrato.

116 Cfr. AIT, Scatola 2.15, I. Targioni, La prima venuta di Bacco in Toscana e i Brigidini di Lamporecchio. Poema Eroicomico dell'estemporaneo autodidatta Idalberto Targioni, 1924. Su Torrigiani cfr. Bianchi, Massoneria e fascismo, cit., pp. 109-138; F. Conti, Storia della massoneria italiana. Dal Risorgimento al fascismo, il Mulino, Bologna 2003, pp. 284-320; G. Padulo, Dall' interventismo al fascismo, in G.M. Cazzaniga (a cura di), Storia d'Italia, Annali, 21, La massoneria, Einaudi, Torino 2006, pp. 657-700.

117 I. Targioni, Calendario agricolo fascista, anno 1930, 8. E. F., vade mecum del contadino Toscano, Tip. Fascista, Firenze 1930, p. 3. Su Picchi cfr. Palla, Firenze, cit., adnomen; Bianchi, Donne di Greve, cit., ad nomen. 
Ha lavorato pel Fascismo dal 1916 dando un calcio a tutto il suo passato, incurante dell'odio malevolo delle masse imbestiate, le sfidò a contraddirlo e, quando ciò avvenne, le sgominò e non seppero rispondergli che con l'insulto e l'imprecazione infecondi. Egli, l'auto-didatta, pugnò e prese di fronte gli ubriachi, i folli e delinquenti colla parola, colla penna e colla persona. [...] Ora il Targioni è finito s'è esaurito nel lavoro, è logorato dal male che lo affligge, non può più lavorare. Il Partito Fascista non può gettarlo, non deve, come un limone spremuto! [...] Oggi che il Duce, con la sua vista d'aquila, vede nel rinnovato amore alla Terra Madre riposto l'avvenire della Patria, [...] ben venga questo libro che parlerà agli agricoltori non solo di lavori campestri, di fiere e di mercati, ma dei loro doveri verso Dio, verso la Patria e verso la Famiglia ${ }^{118}$.

In effetti, per ogni mese il Calendario presentava testi dedicati a Dio (gennaio), alla patria (febbraio), alla famiglia (marzo), ma anche all'igiene della casa, del podere e dell'alimentazione (aprile, maggio, giugno), a educazione e istruzione (luglio), sindacati e corporativismo (agosto), a giovani e vecchi (ottobre), ai boscaioli (novembre) e infine a «Operai, Braccianti Specializzati e Promiscui», la «classe» «che ha nicchiato più di tutte le altre prima di decidersi a entrare nei Sindacati Fascisti» (dicembre). L'introduzione al mese di settembre era invece dedicata ai soli braccianti, il cui numero non era «precisabile nemmeno approssimativamente», «3 o 4 milioni di operai», una «grande massa di rudi, forti e generosi lavoratori»:

Quello che hanno fatto in passato è scusato, anzi è perdonato, ma non lo debbono dimenticare, non debbono dimenticare il vile e astuto inganno teso loro dai partiti democratici e sinistroidi che andavano dal colore bianco al bigio più opaco, al rosso attenuato e scarlatto e al nero fumo comunista e anarcoide. Non debbono dimenticare come fu loro insegnato a bestemmiare la patria, offendere, malmenare ed anche uccidere i fratelli, gli uomini intellettuali della scienza, della saggia politica e della fede religiosa. E non debbono dimenticare quanti hanno preso parte a rappresaglie odiose, alle sopraffazioni materiali e verbali e fu clemente verso di loro Benito Mussolini che poteva punirli e vendicarsi e non lo fece. [...] Ricordino [...] gli assalti alle fattorie e alle altre proprietà [...]. Ma ricordino anche i signori Proprietari! Si ricordino i tempi quando imperava siffatto bolscevismo e si ricordino come pativano e supplicavano perché fosse scongiurato il pericolo. Si ricordino che gli abbiamo difesi per salvare la loro pelle, i loro beni e la loro proprietà, che siamo andati incontro alla morte per loro.

Targioni chiudeva il Calendario ripercorrendo «Cose da non dimenticare» del primo dopoguerra, con uno spazio particolare ai 'fatti di Empoli' del primo marzo 1921, ovvero a una serie di «fattacci» causati dalla «malattia» alimentata dal «vento slavo» e dall'«odio antifascista e antitaliano» ${ }^{119}$.

118 Targioni, Calendario, cit., pp. 7-8.

119 Ivi, p. 135. 
Ma se i «signori Proprietari» non ricordavano, anche i rapporti di Targioni con gli apparati di partito si erano complicati ormai da alcuni anni ed erano segnati da accuse di tradimento e inchieste interne. Mentre in pubblico Targioni presentava riaffermazioni formali della propria onorabilità e fede fascista, in privato Targioni non esitava a ipotizzare vie di uscita dal Pnf. Lo dimostra la corrispondenza del 1928 con Velia, ma il dato emerge già in una lettera del 3 maggio 1924 indirizzata alla stessa figlia. In quella occasione, il padre informò Velia che il Consiglio di disciplina e il Direttorio della Federazione fascista avevano accolto il suo ricorso contro l'espulsione dai sindacati fascisti:

anche questa (che non è la prima e forse non sarà l'ultima pugnalata inferta alla mia dirittura morale e politica) è finita con una sconfitta del mio avversario. Ora la deplorazione non sempre sincera degli amici veri e falsi si converte in auguri e congratulazioni. Cose di cui non so cosa farne, fatte s'intende le debite eccezioni, essedo certo che anche questa volta debba pressoché tutto a me stesso se sono riuscito a superare la difficilissima prova. Già mi si stanno facendo offerte piuttosto lusinghiere, ma io conosco ormai troppo bene il mondo e non mi lascerò facilmente adescare dalle grandi promesse, $\mathrm{Se}$ potrò crearmi una posizione indipendente la preferirò a tutte le altre ${ }^{120}$.

Il vecchio mondo era scomparso. L'«Usignolo» del Montalbano non cantava più. In compenso, il «Pentito» ogni tanto scriveva lettere di delazione e desiderava ardentemente cancellare il proprio passato: «Il Targioni - aveva già scritto anni prima usando, come sempre, la terza persona - non ci tiene a passare per poeta improvvisatore, anzi, se potesse, vorrebbe distruggere quel passato e più ancora molti dei versi che in vent'anni di carriera artistica declamò e scrisse cantando quello che a lui parve il più grande degli umani ideali» ${ }^{121}$.

All'indomani dei Patti lateranensi, un Targioni sempre più amareggiato avrebbe rivendicato ancora una volta il suo ruolo nel fascismo della prima ora con una lettera indirizzata alla direzione dei Sindacati fascisti di Firenze:

Fondai la Sezione Fascista di Lamporecchio, della quale fui nominato segret. p. [politico]. Sfidai i bolscevichi con la stampa e colla parola, con grave pericolo per la mia vita. [...] Feci fiere di beneficenza e raccolsi 65 mila lire recandomi di casa in casa per parlare al popolo perché pagasse almeno la $1 / 2$ della tassa di famiglia [...]. A Lamporecchio, in casa mia, si facevano continuamente le adunanze e le riunioni per fronteggiare i socialcomunisti. La sede del Fascio era in casa mia [...]. Due volte ho sfidato la morte. La prima volta nel 1919 nel Teatro di Lamporecchio. Era la sera di Pasqua allorquando fu preso d'assalto il teatro comunale da circa 400 socialcomunisti scalmanati e ubriachi [...]. L'anno successivo nell'occasione di una tornata consiliare dai socialcomunisti [...]

120 AIT, Scatola 4.2.30, Targioni a Velia, 3 maggio 1924. Cfr. AIT, Scatola 4.1.15, Targioni a Velia, 7 settembre 1928; AIT, Scatola 4.2.38, Targioni a Velia, 11 settembre 1928.

${ }^{121}$ AIT, Scatola 3.22, Idalberto Targioni. Educatore e organizzatore, cit. 
la parola d'ordine era questa: se fossi entrato nella sala sarei stato accerchiato e linciato [...]. Nel 1924 [...] mi recai di casa in casa per la raccolta dei fondi occorrenti per l'erezione del Monumento ai caduti [...] Durante il periodo elettorale del 21 corsi la provincia in ogni posto, dalle montagne pistoiesi ai confini di Volterra [...]. Nell'Empolese, poco dopo il massacro dei marinai, tenni numerose conferenze. C'era l'ordine di accompagnarmi con un camion carico di fascisti [...]. Non ho avuto l'onore di versare il mio sangue nelle trincee di guerra, l'ho versato e ho corso pericolo di vita a correre le strade d'Italia per fare opera sindacale fascista.

La lettera si concludeva denunciando due «massoni» che si erano infiltrati nel partito locale «per rovinarlo» ${ }^{122}$. L'interventismo e il fascismo, insomma, avevano divorato i propri figli. Per Targioni anche il vecchio legame col caro amico di gioventù, il volontario di guerra e poi Gran maestro Domizio Torrigiani, che nel 1929 era confinato a Ponza, doveva essere archiviato.

Va detto che negli anni del regime Targioni non fu l'unico a mettere in rilievo il proprio ruolo svolto nella Rivoluzione fascista, come testimoniano le pubblicazioni di memorie e ricordi di squadristi (della prima o dell'ultima ora). Ma è difficile restare indifferenti confrontando i testi del crepuscolare Targioni, fascista incompreso e rancoroso uomo di regime, con quelli del radioso e promettente «Usignolo» di fine Ottocento o dello spumeggiante e irriverente «Diavolo rosso» d'inizio secolo.

Dalla metà degli anni Venti, dunque, l'ormai stanco Targioni era scomparso di scena. Ritirato quasi completamente alla vita privata, segnato da cattive condizioni di salute, era tormentato da un'ulcera allo stomaco: una malattia emblematica, come se nel corso della sua vita avesse dovuto inghiottire un po' di tutto, di certo non solo il vino acido bevuto stornellando in gioventù, senza poi riuscire a elaborare il proprio vissuto a dispetto delle centinaia, forse migliaia di pagine manoscritte e rielaborate con la macchina per scrivere cercando di dare ordine e senso a una propria storia iniziata con un mistero mai disvelato. Il vecchio Targioni divenne sempre più pessimista e disilluso dalla vita. «Il tempo passa rapidamente e s'invecchia», scrisse nel 1928; «i ragazzi non comprendono la tristezza della vita quando si arriva ad una certa età ${ }^{123}$.

Stando alla sua corrispondenza e agli appunti nei taccuini, trovava un po' di conforto nell'affetto dei familiari e - oramai - in una fede nella divina provvidenza: «Tutta la mia soddisfazione ed ogni gioia di vivere la trovo nel lavoro [...]. Credo la Provvidenza ci penserà. Desidero di vivere non per arricchire, ma per lavorare umilmente e onestamente. E Dio vedrai che ascolterà la mia voce. Avanti dunque e coraggio ${ }^{124}$. Ma anche questo nuovo credo

122 AIT, Scatola 3.23.47, Ill.mo Sig. Professor Bonfatti, cit.; cfr. ivi il manoscritto, per il Prefetto di Firenze, Rapidi cenni della mia opera Fascista dal 1916 al 1929.

123 AIT, Scatola 4.1.14, Targioni a Velia, $1^{\circ}$ settembre 1928.

124 AIT, Scatola 4.1.68, Targioni a Velia, 27 aprile 1927. 
sembrò venire sopraffatto dal pessimismo: «Ormai non resta che rassegnarsi. Sperar di guarir dopo 29 anni di malattia cronica sarebbe illusione...» ${ }^{125}$.

Fiaccato dal progredire della malattia, dalle difficoltà economiche e deluso dallo scarso riconoscimento ottenuto dagli apparati fascisti per il lavoro svolto presso i sindacati per l'agricoltura, a 62 anni Targioni morì a Lamporecchio il 25 maggio 1930.

Il funerale venne fatto con una cerimonia religiosa celebrata da don Luigi Cioletti, forse su richiesta di Targioni. Era passato troppo tempo dal Testamento redatto in versi dal «Diavolo rosso» nel 1901, quando aveva chiesto di essere trasportato con in mano e all'occhiello «un bel mazzo di garofani rossi», senza la presenza di «ceri né incappati» o di un prete «nero», «brutto ed infesto», invitando poi tutti ad andare «a spasso ed a saltare sui prati cantando in coro l'inno di Filippo Turati» ${ }^{126}$.

Nell'Archivio Idalberto Targioni si trova una fotografia che con molta probabilità venne scattata dall'alto di una finestra durante la processione funebre nel centro del paese. Non avendo potuto verificare con certezza la data dello scatto, ho deciso di non riprodurla in questo volume. Ma nell'immagine si notano decine di persone accalcate ai fianchi di un corteo aperto da ragazzini, molti con abiti neri, e subito dietro donne quasi tutte vestite di scuro, con eleganti cappelli in testa e mazzi di fiori in mano, seguite da uomini in giacca e cravatta o con divise di tipo fascista; poi altri uomini che portano labari listati a lutto, in un giorno di sole primaverile ${ }^{127}$.

Dopo il funerale, Targioni cadde nell'oblio.

Penso che la prima causa della sua paralisi fu un gran dispiacere che ebbe alla federazione quando cercarono di allontanarlo dai suoi sindacati - scrisse Sofia a Velia -. Aveva tanto lavorato per i suoi organizzati e quanti sacrifici di giorno e di notte. La ricompensa (nel tuo posto ci deve venire un altro). Povero Berto!! E dire che sei morto con questa spina nel cuore; ed ora noi come faremo a lottare con questa gente? Speriamo! La tua santa memoria, il ricordo di tutte le tue sofferenze mi darà coraggio, non fosse altro avrò il coraggio che dà la disperazione e cercherò in tutti i modi di far valere i tuoi diritti ${ }^{128}$.

Come abbiamo visto, sarebbe stato necessario superare una nuova guerra mondiale, ottenere la Liberazione e costruire la Repubblica per creare le condizioni affinché si iniziasse a scrivere la storia di Targioni, prendendo le mosse con un saggio sulle poesie del «Diavolo rosso» pubblicato negli anni Cinquanta $^{129}$.

125 AIT, Scatola 4.2.23.8, Targioni a Velia, Pontassieve, 23 maggio 1929.

126 Targioni, Il Canzoniere, cit., pp. 88-94; Poli, Lamporecchio, cit., p. 117.

127 AIT, Scatola 4.2.

128 AIT, Scatola 4.2.82, Idalberta e Sofia a Velia, 7 luglio 1930.

129 Cfr. Guerrini, Un poeta estemporaneo, cit. 
Oggi, in un contesto storico e storiografico completamente diverso, privi di progetti sociali o politici di ampio respiro, gli storici e le storiche possono tornare a studiare con nuovi strumenti e carte inedite la biografia pubblica e intima del nocentino, del poeta contadino e del socialista, del patriota, dello squadrista, del fascista deluso. Ovvero del Targioni che seppe decantare tanto la dignità degli ultimi quanto il gusto dei brigidini $i^{130}$, che riuscì ad appassionare i lavoratori semianalfabeti per un futuro dell'umanità che in quegli anni poteva sembrare tutto ancora intero, pieno di potenzialità e con tante storie ancora da fare e da vivere; ma anche la biografia del Targioni che si fece sedurre dalla Grande guerra, che seguì Mussolini dall'interventismo alla conquista del potere e che infine mori accompagnato da rancori e pentimenti ${ }^{131}$.

$\grave{E}$ un lavoro da sviluppare e spero che questo libro possa offrire una base per le prossime ricerche. Perché la ricchezza di quel passato, che con le sue luci e le sue ombre va affidato alla storia, può aiutarci a capire il presente: sia interrogandoci sulle contraddizioni di un contesto politico e sociale cangiante e che sembra difficile da interpretare, sia cercando di superare lo scollamento tra politici, intellettuali e mondo del lavoro, magari con l'ambizione di ritrovare registri concettuali e linguistici comuni capaci di guardare verso il futuro.

130 Cfr. AIT, Scatola 2.17, I Brigidini di Lamporecchio.

131 Cfr. A. Lyttelton, La conquista del potere. Il fascismo dal 1919 al 1929, Laterza, Roma-Bari 1982. 


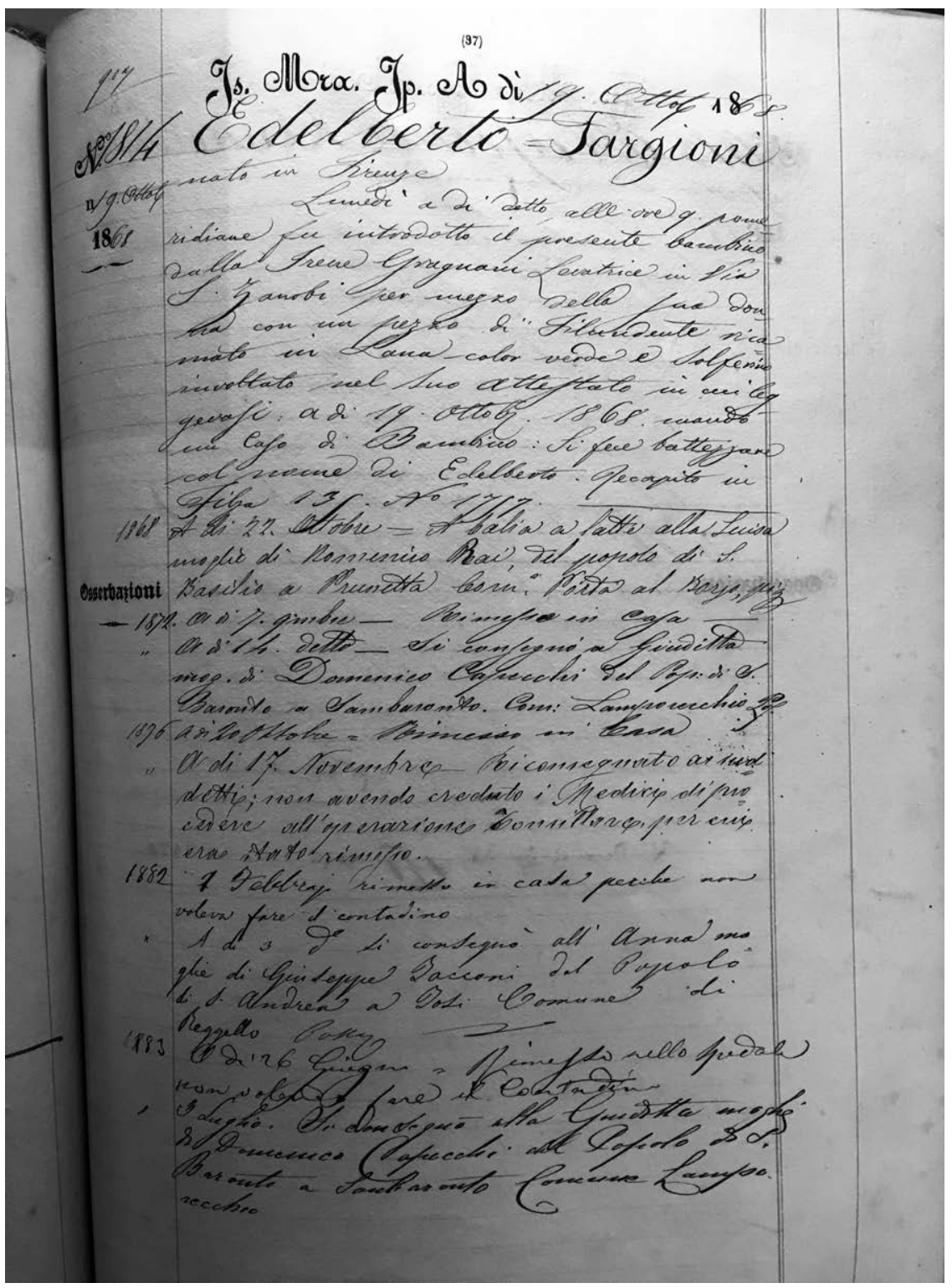

Figura 1 - Il registro dell'Istituto degli Innocenti (AOIF, Balie e Bambini 1868, F8a, p. 4a, 1.10.1868-31.12.1868, n. inv. 786, n. d'ordine 1814). 


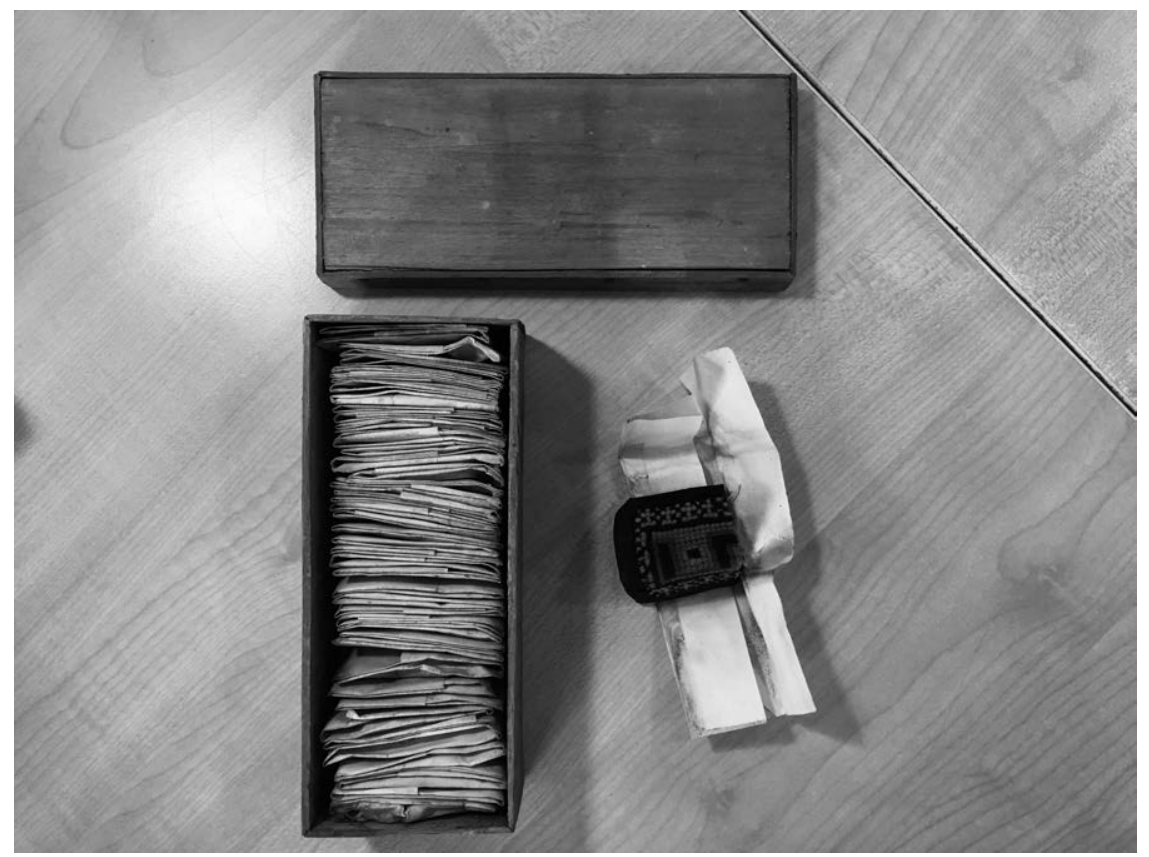

Figura 2 - Il segnale di riconoscimento e il suo contenitore (AOIF, Segnale di riconoscimento 1868, ottobre II parte, M 1814). 


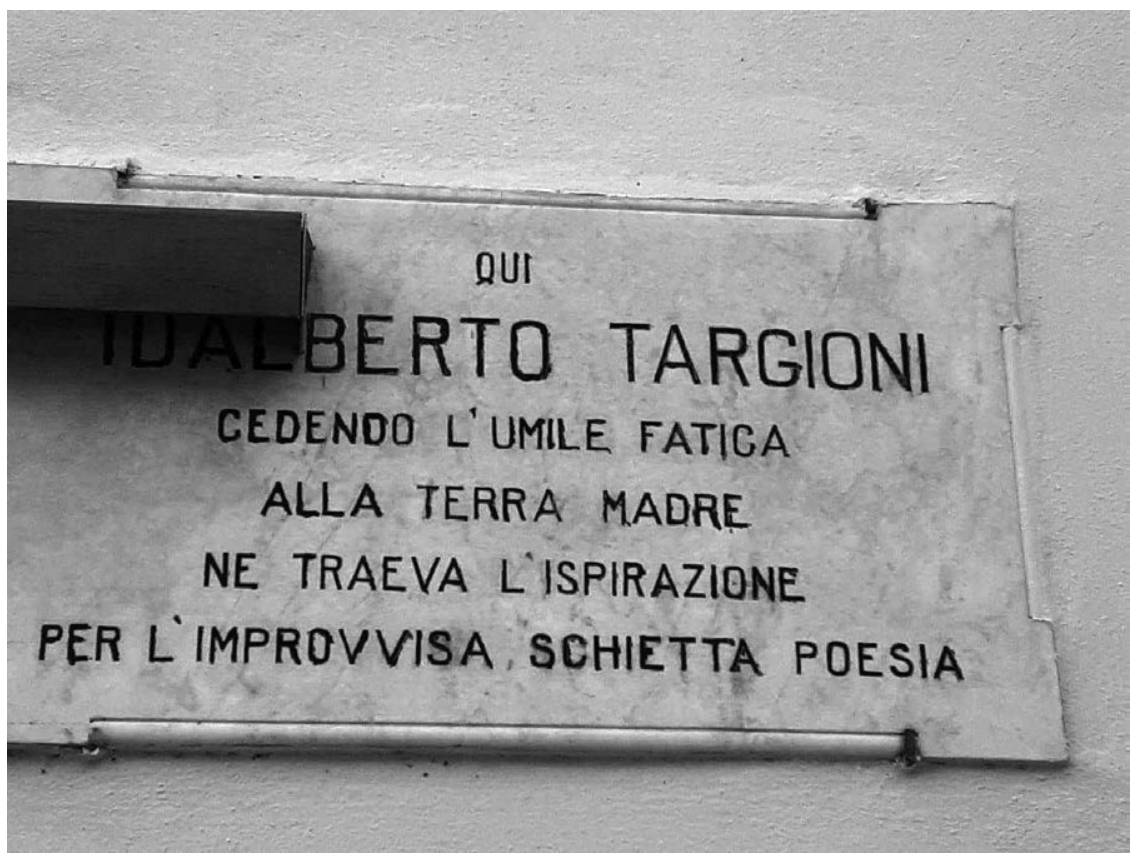

Figura 3 - La targa sulla casa di San Baronto. [Roberta Niccoli e Daniele Bartalucci] 


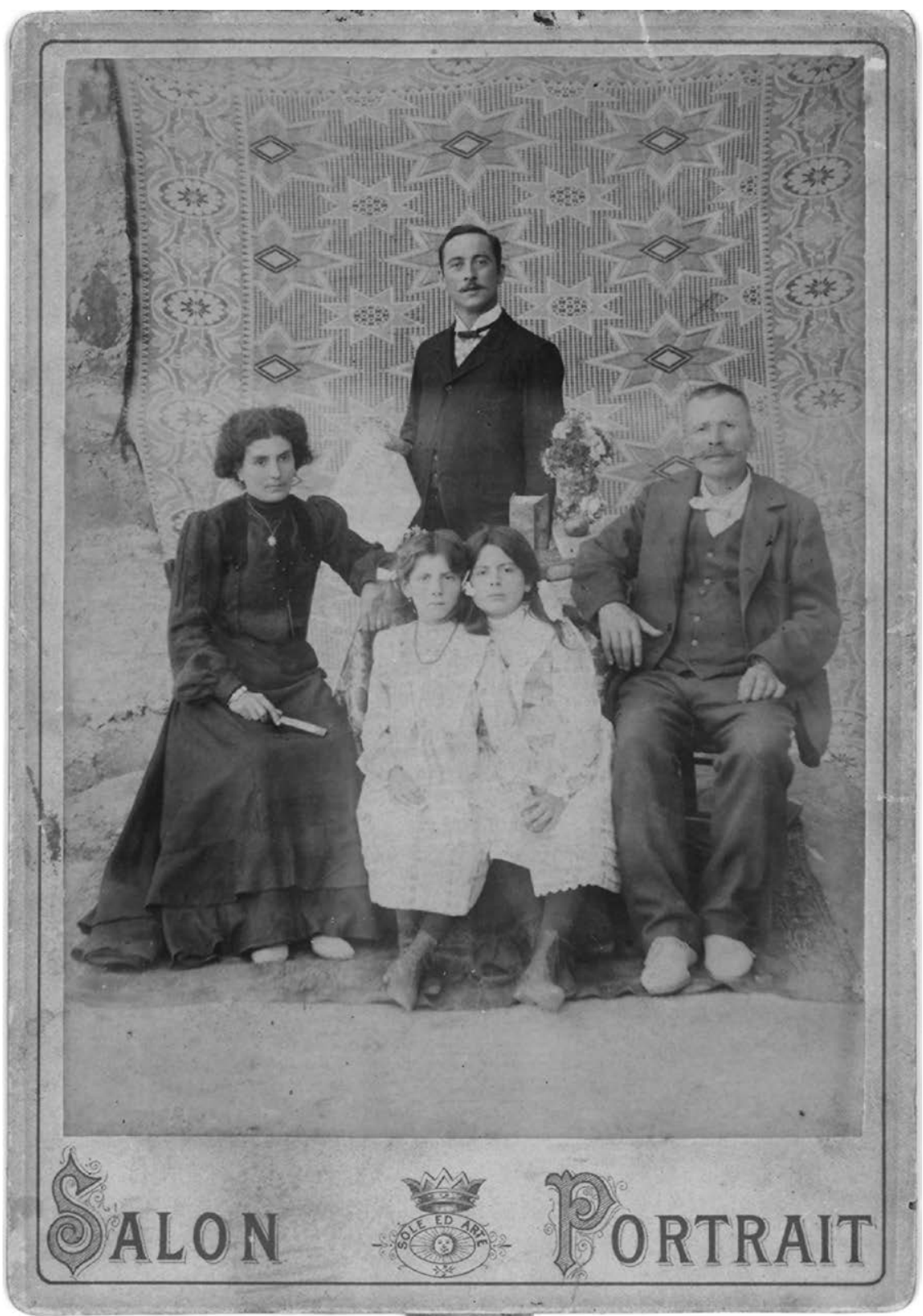

Figura 4 - Idalberto Targioni (al centro) con la moglie Amelia, il suocero Leopoldo Vannacci, le figlie Rita (a sinistra) e Velia (a destra), primi anni del Novecento; si noti il tessuto collocato come fondale sulla parete di casa. [Archivio Idalberto Targioni] 


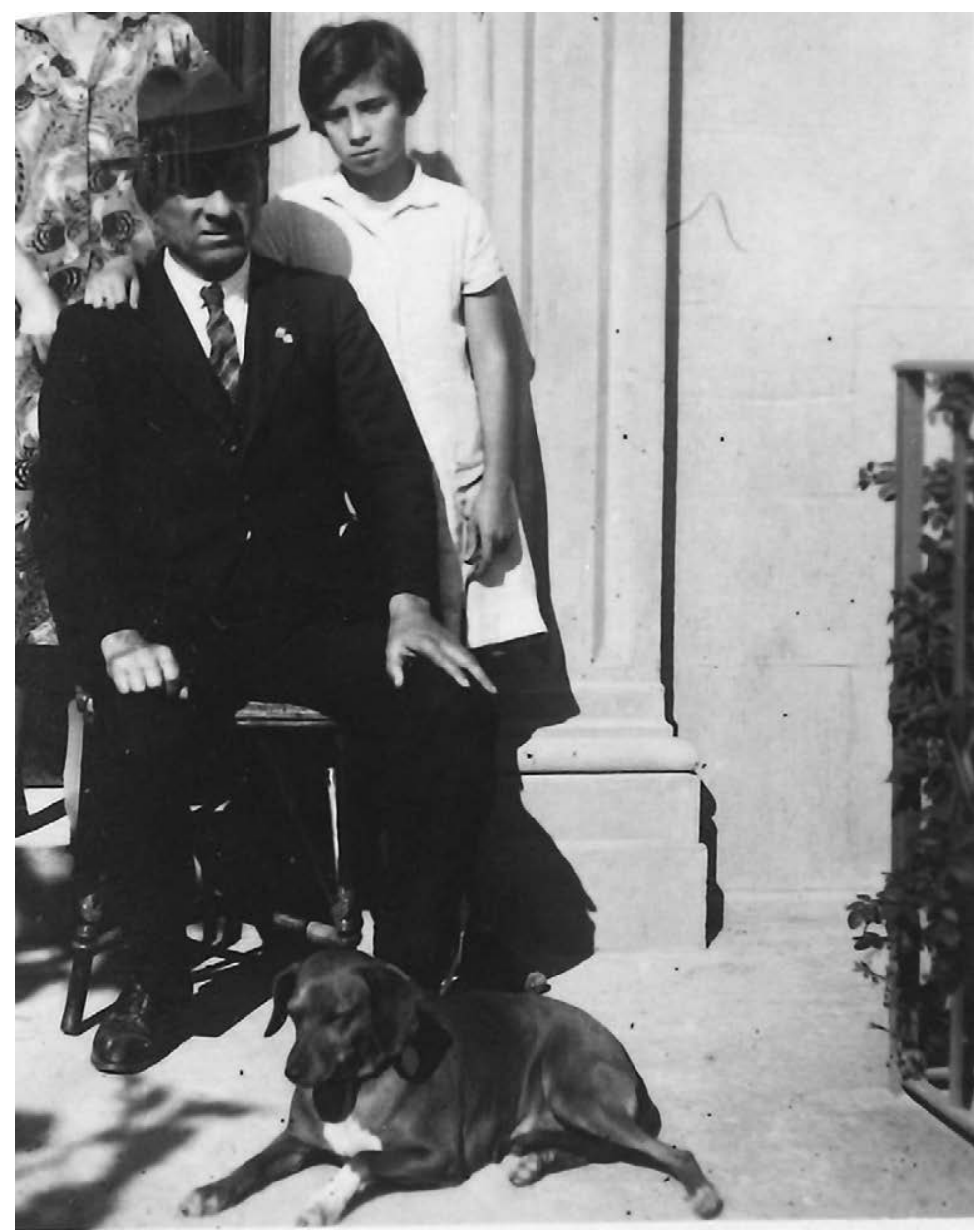

Figura 5 - Idalberto Targioni con la figlia Idalberta, anni Venti; dalla fotografia venne tagliato il volto di Sofia Bacarelli. [Archivio Idalberto Targioni] 



\section{L'Archivio Idalberto Targioni}

a cura di Daniele Lovito

\section{Presentazione}

Il fondo Idalberto Targioni (1868-1930) è stato rinvenuto, alcuni anni or sono, a Lamporecchio (PT), presso la casa appartenuta a una delle nipoti del poeta contadino, Lida Chiappini, ed è qui che, di recente, è stato sottoposto a una prima fase di studio e di riordino. Non avendo alcuna conoscenza pregressa circa la quantità, lo stato di conservazione, la tipologia e il contenuto del materiale documentario effettivamente presente in loco, si è dunque deciso di procedere allo spoglio sistematico di tutti i documenti e alla stesura di un relativo elenco di consistenza, ritenendo che tali operazioni fossero il necessario atto preliminare da compiere in funzione di eventuali, successivi interventi di natura archivistica (sistemazione fisica della documentazione e inventariazione) e storiografica.

Da questa prima ricognizione risulta che il fondo è costituito da 1.391 unità, tra documenti (856) e opere a stampa (535, compresi i 197 volumi della biblioteca di Targioni inseriti in appendice) $)^{1}$. Il materiale ivi conservato si distingue per la grande varietà documentaria: corrispondenza con familiari, amici, compagni di partito, istituzioni; copie di lettere a vari destinatari; taccuini e quaderni con appunti (elenco spese, appuntamenti), bozze di poesie, pensieri, discorsi politici e raccolte di poesie inedite; pagine e ritagli di articoli di giornale scritti da o riferiti a Targioni; fotografie, fonti edite di diverso genere (giornali, opuscoli, manifesti, volantini), una biblioteca ed effetti personali. Di estremo interesse è inoltre un'autobiografia dattiloscritta, cominciata nel 1920 , non portata a compimento e mai data alle stampe. Da rilevare è anche la presenza di una corposa documentazione prodotta in seno al Fascio di Com-

1 Laddove presenti, le copie di una stessa opera a stampa sono state escluse dal conteggio. 
battimento di Lamporecchio (libro cassa, verbali delle adunanze, domande d'ammissione, corrispondenza), di cui Targioni è stato Segretario negli anni 1920-1922, e confluita nell'archivio personale di quest'ultimo.

Gran parte della documentazione è stata prodotta nel periodo 1895-1930. Nel caso delle pubblicazioni a stampa (libri, giornali, opuscoli), si sono, naturalmente, riscontrati dei casi di datazione anteriore o posteriore all'arco cronologico sopraindicato.

Ogni elemento documentario è stato, dunque, analiticamente descritto, indicando, per ognuno, le seguenti informazioni:

- il titolo, laddove presente. Se mancante, è stato riportato l'incipit del testo (in corsivo, seguito da punti di sospensione) o una breve descrizione riassuntiva del suo contenuto;

- la tipologia del documento (lettera, minuta, cartolina, opuscolo, giornale ecc.);

- il tipo di materiale (a stampa, manoscritto, dattiloscritto). Tale informazione è stata tralasciata per i periodici, per i quali si dà per scontata l'edizione a stampa;

- la data topica e cronica (dove e quando è stato prodotto il documento). Se assenti, in alcuni casi, il compilatore ha inserito tra parentesi quadre il nome del luogo e gli estremi cronologici in cui il documento è stato verosimilmente prodotto;

- $\quad$ il numero di carte (per i documenti) o di pagine (per le opere edite);

- una breve descrizione dell'oggetto e del suo contenuto.

Per ragioni di economia del testo è stata omessa l'indicazione relativa alla presenza di carte intestate, a meno che il nome dell'autore fosse assente o illeggibile e quindi l'intestazione abbia fornito informazioni suppletive circa la sua identità e, in alcuni casi, circa la data approssimativa in cui il documento è stato prodotto.

Una volta descritto in tutti i suoi elementi, il corpus documentario è stato poi diviso per tipologia e contenuti e sistemato in otto scatole distinte. Al momento, dunque, si è deciso di non stilare un vero e proprio inventario, ma piuttosto un qualcosa che sta nel mezzo tra il sintetico elenco di consistenza e il più analitico e ponderato strumento archivistico che è, appunto, l'inventario. In futuro, però, non si escludono ulteriori interventi di natura archivistica, come la creazione di serie e sottoserie e una conseguente nuova sistemazione del materiale.

È necessario, inoltre, dire che non è stato possibile rintracciare elementi utili per ricostruire l'ordine originario dei materiali, a causa di vari interventi cui, nel tempo, sono stati sottoposti. Il complesso di carte prodotte e appartenute a Targioni, infatti, dopo la sua morte passò, prima, nelle mani della figlia Velia e, poi, in quelle della nipote Lida, figlia di Velia e Torello Chiappini. Tutto ciò ha dunque contribuito a rendere il complesso di carte un archivio 
'improprio' o comunque una realtà intermedia tra un archivio e una raccolta. Di Lida, in particolare, si notano alcuni interventi di risistemazione dei documenti, come, ad esempio, l'inserimento di alcuni di questi in cartelline di cartone o l'inclusione, nel corpus documentario, di documenti postumi, fotocopie di opere edite del Targioni e di trascrizioni dattiloscritte di alcune sue poesie.

$\grave{E}$ doveroso, infine, precisare che la scelta di accludere al fondo, e in appendice al relativo elenco di consistenza, i tomi facenti parte della biblioteca privata di Idalberto Targioni (scatole 6, 7,8) è stata dettata principalmente dalla volontà di fornire al fruitore ulteriori elementi per capire quali furono alcuni dei testi che contribuirono alla formazione culturale e letteraria di un personaggio dalle molte sfaccettature e dalla complessa personalità ${ }^{2}$.

\section{Elenco di consistenza}

\section{Scatola 1}

\section{Consistenza: 14 docc., 17 op. a st.; 52 cc., 689 pp.}

La scatola è composta prevalentemente da opere edite di Idalberto Targioni (IT) e da copie dattiloscritte e fotocopiate da terzi di alcune di esse o di porzioni di esse.

1. IT, foto-cartolina postale, s.l., s.d. [1920-1930], 1 c. Sul recto, foto in bianco e nero raffigurante IT in età matura.

2. Biglietto da visita, cartoncino stampato, s.l., s.d., $1 \mathrm{c}$.

3. IT, Canzoniere di poesie popolari, opuscolo, Pistoia, Tip. Flori e Biagini, $1895,56 \mathrm{pp}$. (errata corrige, probabilmente autografo, a p. 56).

4. IT, Il canzoniere. Poesie sociali, opuscolo, Empoli, Tip. E. Traversari Edit., 1902, 96 pp., 2 copie (una delle quali senza copertina).

5. Quaderno verde con ritagli di opere edite inerenti la polemica tra IT e Luigi Manuelli, s.l., 1902, 18 cc.:

1. Polemica Targioni Manuelli, 13 pp.

2. Ultima risposta del Manuelli al Targioni, $13 \mathrm{pp}$.

2 Quella del Targioni potrebbe a tutti gli effetti essere considerata una 'biblioteca d'autore' in quanto potrebbe fornire a chi la consulta un importante strumento interpretativo e di conoscenza capace di documentare i percorsi di lettura, la formazione, il contesto culturale del suo possessore. Una 'biblioteca d'autore', infatti, da definizione, «indica una raccolta libraria privata e personale che, per le sue caratteristiche interne, tramite i singoli documenti e nell' insieme della collezione, sia in grado di testimoniare l'attività intellettuale, la rete di relazioni, il contesto storico culturale del suo possessore». Cfr. Giuliana Zagra, Biblioteche d'autore, <http://www.aib.it/aib/cg/gbautd07.htm3> (05/18). 
3. Terza Risposta del Targioni al Manuelli, 11 pp.

6. IT, Terza Risposta del Targioni al Manuelli, opuscolo, Firenze, Tip. E. Sevieri, 1902, 16 pp.

7. IT, Il prete attraverso le religioni e la Storia. Poemetto eroicomico in ottava rima, opuscolo, Firenze, Tip. Polli, 1906, 20 pp.

8. IT, Agli emigrati italiani, opuscolo, Zurigo, Tip. L. Migliorini, 1908, 8 pp.

9. IT, Dalla nascita alla morte della terra: Conferenza in versi, tenuta la prima volta all'Università popolare di Zurigo il 10 Maggio 1909, opuscolo, Pistoia, Tip. Fratelli Ciattini, 1909, 16 pp., 2 copie.

10. IT, Il terremoto calabro-Siculo: Bozzetto sociale in versi martelliani, opuscolo, Pistoia, Tip. Fratelli Ciattini, 1910, 16 pp., 2 copie.

11. IT, Civiltà borghese o tirannide capitalistica?, opuscolo, Firenze, Tip. U. Polli, 1911, 8 pp., 3 copie.

12. IT, Canzoniere di poesie sociali, opuscolo, Firenze, Tip. U. Polli, 1912, 110 pp., 3 copie (due senza copertina originale).

13. IT, I miei Ricordi d'infanzia, ovvero I proverbi di mamma Giuditta, opuscolo, Firenze, Casa Ed. Polli-Targioni, 1912, 30 pp., 2 copie.

14. IT, Piccoli Parassiti. Opuscolo di propaganda dedicato agli operai, opuscolo, Firenze, Tip. U. Polli, 1912, 16 pp.

15. IT, La conferenza di Damiano agli elettori del Collegio di Empoli, opuscolo, pubblicato a cura del Comitato di Propaganda Elettorale Socialista, Empoli, Tip. Traversari, 1913, 12 pp., 2 copie.

16. IT, La conflagrazione europea. Poema antiguerresco, opuscolo a stampa, Firenze, Tip. U. Polli, 1914, 42 pp., 25 copie.

17. IT, Satira dedicata al piu grande farabutto dell'Universo. Annibale Palandri di S. Pierino, manifesto a stampa, s.l., s.d., 2 pp. Venticinque strofe in ottava rima di satira, stampate su foglio di grandi dimensioni e dedicate alla figura di Annibale Palandri, nativo di S. Pierino di Pistoia e truffatore di professione.

18. IT, Calendario agricolo fascista. Anno 1930 - VIII E. F. Vade mecum del contadino Toscano, opuscolo, Firenze, Tip. Fascista, 1930, 150 pp., 48 copie.

19. IT, Vent'anni di propaganda e cinque anni d'Amministrazione Socialista nel Comune di Lamporecchio, opuscolo, Pistoia, Tip. Guido Grazzini, 1920, 32 pp., 3 copie.

20. IT, La conflagrazione europea. Poema antiguerresco, opuscolo fotocopiato, Firenze, Tip. U. Polli, 1914, 42 pp. fotocopiate su 26 pp. formato A4.

21. Estratto da I proverbi di mamma Giuditta, carta datt., s.l., s.d., 11 cc., 2 copie (una in fotocopia). Trascrizione delle pp. 12-32 dell'opuscolo originale.

22. La Favola dei Topi, fotocopia di datt., s.l., s.d., 2 cc. Opera di IT trascritta da terzi. 
23. Mentresta nevicando, carta datt., s.l., s.d., 2 cc., 2 copie (una in fotocopia). Componimento di IT, scritto da terzi ed estratto da Canzoniere di poesie sociali.

24. Caprera, carta datt., s.l., s.d., 2 cc., 2 copie (una in fotocopia). Componimento di IT, scritto da terzi ed estratto da Canzoniere di poesie sociali.

25. La vendemmia, carta datt., s.l., s.d., 2 cc., 2 copie (in fotocopia). Componimento di IT, scritto da terzi ed estratto da Canzoniere di poesie sociali.

26. La sementa, fotocopia di datt., s.l., s.d., 1 c., 2 copie. Componimento di IT, scritto da terzi ed estratto da Canzoniere di poesie sociali.

27. La pace, fotocopia di datt., s.l., s.d., 2 cc., 2 copie. Componimento di IT, scritto da terzi ed estratto da Canzoniere di poesie sociali.

28. Maggio 1911, carta datt., s.l., s.d., 2 cc., 2 copie (in fotocopia). Componimento di IT, scritto da terzi ed estratto da Canzoniere di poesie sociali.

29. Sopra il contrasto del nuovo tronco di strada Piastre-Prunetta, fotocopia di datt., s.l., s.d., 3 cc. Componimento di Pietro Pisaneschi in 15 strofe in ottava rima, scritto da terzi.

30. Qui per scienza ci vorrebbe un Dante..., fotocopia di carta datt., s.l., s.d., 3 cc. Componimento di Pietro Pisaneschi in 14 strofe in ottava rima, scritto da terzi.

31. Composizione in ottava rima del poeta I. Targioni sul tema: l'ignoranza di un popolo di contadini, fotocopie di quaderni mss., s.l., s.d., 57 pp. di quaderno fotocopiate su 32 pp. formato A4, 2 copie. Fotocopie di carte con trascrizione, ad opera di Guido Larini, di una composizione di IT e del contraddittorio in ottava rima tenuto sempre da IT con Fortunato Chiti il 19 marzo 1911 nella Società di M. Soccorso di Varlungo (Firenze) e dal titolo Per l'educazione della donna secondo la morale socialista e secondo la morale cristiana.

\section{Scatola 2}

Consistenza: 240 docc., 32 op. a st., di cui 17 periodici; 1069 cc., 606 pp. La scatola contiene prevalentemente articoli di giornale (molti dei quali scritti da IT), bozze di scritti politici e di poesie inedite, documenti e fotocopie di documenti.

1. Pagine e articoli di giornale raccolti in una cartellina di colore blu, sul cui frontespizio si legge: «Scritti. I. Targioni»:

1. «L'Alleanza. Periodico quindicinale antibolscevico di critica e di controllo per la difesa sociale e degl'interessi agricoli e commerciali dei Comuni limitrofi di Montalbano», Empoli, 31 gennaio 1921, 4 pp. Articoli di IT a p. 1, Nella Chiesa Rossa - Dopo il Congresso di Livorno, e a p. 3, Il nostro socialismo.

2. «L'Alleanza», Empoli, 6 maggio 1921, 4 pp. Articolo di IT a p. 3, Maggio 1921 (Dedicato ai contadini). 
3. «L'Alleanza», Empoli, 13 maggio 1921, 4 pp. Articoli di IT a p. 1, Battaglia Campale - Tutti alle urne! Per la nuova e grande Italia, e a p. 2, 5 maggio 1921. Canto nuovo all'Italia (componimento in versi).

4. Raccolta di ritagli di giornale, incollati su 2 pp., s.l., s.d., recanti i seguenti articoli: Primizie. Conversazioni estive (confronto tra un contadino e un prete, probabilmente estratto da Contrasto tra un prete democratico-cristiano e un contadino ascritto alla Lega di resistenza, a cura della Sez. socialista di Empoli, Empoli, 1902), Camera del Lavoro e Per gli Scavi di Piazza del Duomo, firmato «Il Monello Pistoiese».

5. «Giosuè Carducci. Numero unico a benefizio del Consorzio dei Patronati scolastici», Firenze, 3 marzo 1907, 4 pp.

6. La casa, carte mss., s.l. s.d. [1921-1930], 4 cc. Saggio sulla mancanza d'igiene nei nuclei abitativi dei contadini.

7. Bozza de La casa, carte mss., s.l. s.d. [1921-1930], 3 cc.

8. La Patria, carte mss., s.l. s.d. [1921-1930], 4 cc. Carte preparatorie per un saggio dedicato all'esaltazione dell'Italia dal punto di vista naturalistico, storico, artistico e letterario. Una terra che ha saputo riscattarsi dal secolare giogo straniero e che adesso deve difendersi dai nemici interni.

9. Bozza de La Patria, carte mss., s.l., s. d. [1921-1930], 1 c. Appunto di cinque righe sul tema della patria.

10. La famiglia, carte mss., s.l., s. d. [1921-1930], 4 cc. Saggio dedicato al tema della famiglia quale nucleo fondamentale della società umana.

11. Discorso sul grano. Parole pronunziate da IT in la mattina del 2810-1925, quadernino ms., [Firenze], [post 28 ottobre 1925], 8 cc. Trascrizione del discorso pronunciato da IT in qualità di Segretario del Sindacato Fascista per l'Agricoltura della Provincia di Firenze, probabilmente a Firenze il 28 ottobre 1925.

12. «L'Avvenire. Organo socialista settimanale del Circondario di Pistoia», Pistoia, 1 maggio 1913, 4 pp. Contiene i seguenti scritti di IT: a p. 1 poesia intitolata Maggio 1913; alle pp. 1-2 articolo intitolato Il grande monito del 1 maggio 1913.

13. Ritagli di giornale sulla guerra europea, incollati su o inseriti tra le pagine di cinque copie del numero 4 di una rivista letteraria di 4 pp. intitolata «Nuove Pubblicazioni», al cui interno sono presentate, con breve descrizione dei contenuti, quattro opere del Targioni (Canzoniere di poesie sociali, I miei Ricordi d'infanzia, ovvero I proverbi di mamma Giuditta, Ettor e Giovannitti, Piccoli parassiti), tutte edite nel 1912. Gli articoli contenuti sono i seguenti:

1. La commemorazione di Pasqua - Il discorso del prof. Salvemini, probabile fonte «Il Nuovo Giornale», Firenze, s.d. [post 11 di- 
cembre 1917], 1 c. Articolo di Ettore Lazzerini sulla commemorazione di Pasquale Villari (deceduto l'11 dicembre 1917) nella sede della Società "Leonardo da Vinci" di Firenze. Messaggio di plauso a Wilson per l'ingresso degli USA nel conflitto mondiale inviato dal prof. Gerolamo Vitelli e dal presidente della "Leonardo" Angelo Orvieto. Discorso di Gaetano Salvemini in ricordo del prof. Villari.

2. Per la Pace nel mondo, probabile fonte «Il Nuovo Giornale», Firenze, s.d. [post 3 marzo 1918], 1 c. Articolo di Guido Podrecca contro la barbarie tedesca e contro la pace di Brest Litowsk (3 marzo 1918).

3. Roberto Ardigó tenta suicidarsi, probabile fonte «Il Nuovo Giornale», Firenze, s.d. [7 febbraio 1918], 1 c. Articolo di Enrico Ferri sul primo tentativo di suicidio del filosofo positivista Roberto Ardigò (1828-1920), avvenuto il 6 febbraio 1918.

4. «Il Nuovo Giornale», Firenze, 22 gennaio 1918, 2 pp. (ritagliate). A p. 2 articolo di Giovanni Papini, Il popolano.

5. L'on. Giovanni Rosadi in Palazzo Vecchio, probabile fonte «Il Nuovo Giornale», Firenze, s.d. [1918], 1 c. Discorso patriottico dell'on. Rosadi (1862-1925) in Palazzo Vecchio.

6. Lettera dal fronte di Arturo Stanghellini allo zio senatore Chiappelli, probabile fonte «Il Nuovo Giornale», Firenze, s.d. [post 1 maggio 1918], $1 \mathrm{c}$.

7. Il comunicato tedesco, probabile fonte «Il Nuovo Giornale», Firenze, s.d. [luglio 1918], 1 c. Sul recto è presente una comunicazione inerente alcune operazioni di guerra delle truppe tedesche sul fronte occidentale. Sul verso v'è un estratto del discorso di un parlamentare italiano alla Camera dei Deputati circa l'andamento della guerra.

8. La querce schiantata, probabile fonte «Il Nuovo Giornale», Firenze, s.d. [post 6 ottobre 1917], 1 c. Sul recto v'è un articolo sulla morte del poeta, giornalista e scrittore Edoardo Scarfoglio, deceduto il 6 ottobre 1917. Sul verso, invece, ci sono notizie intorno alla guerra.

9. Appunti e ricordi, [ibidem], 1 c. Articolo su Edoardo Scarfoglio probabilmente ritagliato dalle stesse pagine di giornale da cui è stato estratto La querce schiantata.

10. Parla l'on. Maffi, probabile fonte «Il Nuovo Giornale», Firenze, s.d. [1917-1918], 1 c. L'onorevole Maffi chiede sussidi e sostegno per soldati e loro famiglie. Protesta per trattamento dei prigionieri italiani ammalati. Sul verso v'è l'articolo Sapone gratis per famiglie, in cui si danno consigli per realizzare in casa il sapone di Marsiglia. 
11. Un socialista tedesco propugna la pace per accordi, fonte non riconoscibile, s.l., s.d. [1918], 1 c.

12. «Il Popolo Pistoiese. Organo della democrazia liberale pistoiese», Pistoia, 25 dicembre 1920, 4 pp.

13. «La Stampa», Torino, 16 dicembre 1922, 8 pp.

14. «Avanti! Giornale del Partito Socialista», Milano, 17 gennaio 1917, 2 pp. Sul recto è presente un articolo di Enrico Leone, Filosofia galeotta; sul verso Pro Avanti! Sottoscrizioni del 1917, con un elenco dei sottoscrittori del giornale socialista.

15. «La Nazione», Firenze, 28 settembre 1928, 2 pp. Sul recto è presente un articolo di R. Dalla Volta su L'impiego dei capitali.

16. «La Nazione», Firenze, 26 settembre 1928, 2 pp. Sul recto è presente l'articolo La decadenza demografica dei Paesi d'Occidente documentata in un vibrante articolo del Duce.

17. «La Nazione», Firenze, 2 febbraio 1924,6 pp. Sulla prima pagina: Mussolini esalta l'opera delle "Camice nere" prima e dopo la rivoluzione fascista

18. «Gazzetta di Venezia», Venezia, 30 novembre 1917, 4 pp. Sulla prima pagina "Non piegar d'un ugna" di Gabriele D'Annunzio e notizie Sui campi di battaglia.

19. Contraddittorio tra Fortunato Chiti e IT a Ponte a Elsa, manifesto a stampa, Pistoia, [1911?], 2 pp. Manifesto pubblicitario per il contraddittorio che si tenne al Teatro "Lorenzo Antonini" di Ponte a Elsa tra IT, socialista e anticlericale, e Chiti, conservatore e credente, sui seguenti temi: «Dio e La Scienza / Stato Borghese e Collettivismo».

2. Al mio carissimo cognato Afrisio Vannacci nella fausta occasione delle sue nozze colla signorina Elide Torrigiani in segno d'affetto e stima perenni offro, cartolina a stampa, Pescia, Tip. Cooperativa, 22 ottobre 1904, 2 cc. Cartolina dedicata alle nozze del cognato di IT con poesia dedicatoria all' interno.

3. G. Sarti, Gli ultimi avvenimenti nel popolo di Lecore. Pubblicati per opera e volontà di popolo, opuscolo, Lecore, 16 aprile 1912, 16 pp.

4. Quaderno con aforismi di vari autori, appunti e componimenti inediti di IT, quaderno ms., s.l. [Lamporecchio], s.d. [1894-1895], 21 cc.

5. «Idalberto Targioni. Fotocopie», cartellina verde contenente i seguenti elementi:

1. Il canzoniere: poesie sociali, fotocopia di opuscolo, 96 pp., 3 copie.

2. I miei Ricordi d'infanzia, ovvero I proverbi di mamma Giuditta, fotocopie ingrandite dell'opuscolo originale, 32 pp., 2 copie (una in formato A4).

3. La conflagrazione europea. Poema antiguerresco e Terza risposta del Targioni al Manuelli, fotocopie ingrandite di opuscoli, $42+16$ pp., 3 copie. Unione in fotocopia, ad opera di terzi, di due volumi di IT. 
4. Il canzoniere. Poesie sociali, opuscolo fotocopiato, $96 \mathrm{pp}$.

5. Canzoniere di poesie sociali, fotocopia di opuscolo, $130 \mathrm{pp}$. fotocopiate su 31 pp. in A4.

6. Canzoniere di poesie popolari, fotocopia di opuscolo, $56 \mathrm{pp}$. fotocopiate su 15 pp. in A4.

7. Civiltà borghese o tirannide capitalistica?, fotocopia di opuscolo, 8 pp. fotocopiate su 6 pp. in A4.

8. Al mio carissimo cognato Afrisio Vannacci nella fausta occasione delle sue nozze colla signorina Elide Torrigiani in segno d'affetto e stima perenni offro, fotocopia di cartolina, s.l., s.d., 2 cc.

9. Caprera, fotocopia di datt., s.l., s.d., 3 cc., 2 copie di p. 2. Componimento di IT, scritto da terzi ed estratto da Canzoniere di poesie sociali.

10. La vendemmia, fotocopia di datt., s.l., s.d., 2 cc. Componimento di IT, scritto da terzi ed estratto da Canzoniere di poesie sociali.

11. La pace, fotocopia di datt., s.l., s.d., 2 cc. Componimento di IT, scritto da terzi ed estratto da Canzoniere di poesie sociali.

12. Maggio 1911, fotocopia di datt., s.l., s.d., 2 cc. Componimento di IT, scritto da terzi ed estratto da Canzoniere di poesie sociali.

13. La sementa, fotocopia di datt., s.l., s.d., 1 c. Componimento di IT, scritto da terzi ed estratto da Canzoniere di poesie sociali.

14. Componimento incompleto, fotocopia di datt., s.l., s.d., 1 c.

15. Luisa Martini, In memoria di Idalberto Targioni..., fotocopia di articolo de «Il Segno di Empoli», anno 6, n. 21, aprile 1993, p. 8-9, 2 pp. fotocopiate su una pagina A3.

6. «Nonno», cartellina verdolina usata probabilmente da Lida Chiappini per raccogliere articoli di giornale e documenti appartenuti a suo nonno IT. Gli elementi contenuti sono i seguenti:

1. Biglietto di sottoscrizione per «L'Alleanza», Lamporecchio, s.d., 1 c., 2 copie.

2. Lida Chiappini a IT e risposta, lettera ms., Lamporecchio, 25 dicembre 1927 e risposta 1 gennaio 1928, 2 cc. + fotocopie della lettera. Lida invia gli auguri di buone feste al nonno, che, usando come supporto scrittorio le stesse carte usate da Lida, ricambia gli auguri ricevuti.

3. IT a Velia, lettera ms., Firenze, 30 maggio 1928, 2 cc. IT informa la figlia sul suo stato di salute e che il Segretario Generale ha maturato una buona opinione di lui e che ha in mente di fargli fare un avanzamento di carriera.

4. Busta da lettera rossa vuota, busta, s.l., s.d., 1 c.

5. Busta da lettera arancione inviata dalla Sede del Comando del $3^{\circ} \mathrm{Reg}$ gimento Paracadutisti, probabilmente destinata a Idalberto Chiappini, con foto di IT sviluppate dalla nipote Lida, foto, s.l., s.d. Contiene: 
1. Negativo di foto ritraente IT

2. Foto ritraente IT, 1 c., 2 copie.

3. Cartolina con foto di IT, $1 \mathrm{c}$.

6. «L'Avvenire», Pistoia, 3 dicembre 1911, 4 pp. +3 copie in fotocopia. Articolo di IT alle pp. 1-2, Spulciando il discorso di Giovanni Pascoli.

7. Al Gufo, contribuente non moroso, e all'eletta schiera dei noti bestioni, opera a stampa, Empoli, Tip. Popolare, maggio 1915, 4 pp., 11 copie. Alle pp. 1 e 3-4 sono presenti scritti polemici probabilmente rivolti a colui che, in contrasto con l'operato dell'Amministrazione Targioni sul fronte delle tasse, firmava articoli polemici con l'anonimo appellativo di «contribuente non moroso» e che qui IT ribattezza «Gufo»3.

8. La suocera e la nuora. Scherzo comico in un atto, carte mss., [Lamporecchio], s.d., 8 cc.

9. Elenco del ricavato delle mie accademie dell'anno 1905, appunti su foglio a foglio a protocollo, [maggio-giugno 1905], 2 cc.

10. La Vittoria del prete Leopoldo Naldi di Riglione, manifesto a stampa, Prato, Tip. Cecchi, s.d., 2 pp., 3 copie. Componimento anticlericale di 17 strofe in ottava rima.

11. «La Voce», Firenze, 23 dicembre 1909, 4 pp.

12. «L'illustrazione italiana», Messina dopo la catastrofe del 28 dicembre 1908, rivista illustrata, s.l., s.d. [1908], 16 pp. Foto di una Messina devastata dal terremoto scattate dall'inviato della rivista Angelo Cairoli.

7. Cartellina della Ditta Chiappini - Documenti vari. Bozze di lettere, di scritti poetici e politici, documenti vari:

1. «Numero Unico. Stampato a cura della Sezione Socialista di Lamporecchio per le elezioni amministrative del 12 luglio 1914», Lamporecchio, 1914, $4 \mathrm{pp}$. Appunti di programma politico postbellico, carte mss., s.l. [Lamporecchio], s.d. [1919], 6 cc. Il supporto scrittorio è costituito da 4 copie di una pagina del numero 4 di «Nuove Pubblicazioni» (cfr. 2.1.13).

2. Bollettino elettorale. Lettera-Programma dei Partiti Popolari di Lamporecchio, manifesto a stampa, Pistoia, F.lli Ciattini, 1910, 2 pp. I Partiti popolari di Lamporecchio espongono il loro programma in vista delle elezioni del 31 luglio 1910, valide per l'elezione di 8 consiglieri comunali. A p. 2 è presente una lettera aperta «Ai cittadini liberi di Lamporecchio» firmata IT.

3 Sull'argomento cfr. Vent'anni di propaganda e cinque anni d'Amministrazione Socialista nel comune di Lamporecchio, Tip. Guido Grazzini, Pistoia 1920, pp. 5-7. 
3. Elenco delle mie accademie dell'anno 1906, foglio a protocollo ms., s.l. [Lamporecchio], s.d. [1907], 2 cc. Elenco dettagliato dei guadagni, appuntati mese per mese, ottenuti con le pubbliche accademie di poesia.

4. Chi è piu sensibile il cuore o il cervello? Contrasto in ottava rima, fogli a protocollo mss., s.l. [Lamporecchio], s.d., $8 \mathrm{cc}$. Componimento poetico in ottava rima, diviso in 37 strofe, sul rapporto tra cuore e cervello.

5. Ho letto l'articolo della Farfalla..., foglio a protocollo ms., s.l., s.d., $2 \mathrm{cc}$. Scritto polemico sui matrimoni di convenienza tipici fra i borghesi, nei quali spesso le donne erano costrette a sposarsi non per amore e con uomini ricchi, ma assai più anziani.

6. Come mai sig. Sindaco non mette fuori le bandiere il giorno che c'è il consiglio?..., carta ms., s.l., s.d., 1 c. Appunti di una discussione circa l'approvazione di un piano edilizio fatta nel Consiglio Comunale di Lamporecchio.

7. IT a Ezio [Galeotti, membro del fascio di Lamporecchio], minuta ms., Firenze, 13 ottobre 1922, 1 c. IT informa il destinatario che quella stessa mattina, nella sede della Federazione, il fascio di Cerreto Guidi aveva invitato quello di Lamporecchio a partecipare alla cerimonia di commemorazione del fascista Guido Pacini, ucciso il 16 ottobre 1921.

8. Ad un amico anticlericale. Noi che nascemmo senza ipocrisie, carte legate e mss., s.l., s.d., 40 cc. Raccolta di bozze di diversi componimenti poetici: c. 1, Vittoria Colonna Marchesa di Pescara; cc. 2r-5r, Lucrezia moglie di Collatino, sesto figlio di Tarquinio Re di Roma; cc. 5r-6r, Vita e morte di Giulio Cesare; c. 6r, Francesco Ferrucci; cc. $6 \mathrm{v}-8 \mathrm{r}$, componimento senza titolo; c. 8r, Discorsetto; c. 8v, componimento senza titolo; cc. 9r-10v, Monti e Tognetti; c. 11, Dedicatoria alla Nobile Signor Laura Merrich; c. 12, A Firenze; c. 13, Il fior della vita; c. 14r, Elegia; cc. 14v-15r, A Lamporecchio; cc. 15v-16r, Primavera; c. 16, Le donne di Visberga; cc. 16v-18r, appunti storici sulla vita di Tarquinio; c. 18r, 17 settembre 1891; cc. 18v-20v, Breve compendio della vita di $G$. Garibaldi; cc. $21 \mathrm{r}-22 \mathrm{v}$, bozze di lettere a un tale Attilio; c. 23r, Finché di questa gioventu fugace...; c. 23v, Il mio nascimento; c. 24r, Giuramento; c. $24 \mathrm{v}$, Era d'aprile sulla sera...; cc. 25r-27v, L'Assedio di Torino 1706; cc. 28r-29r, Morte di Anita; cc. 29r-30r, Orazio Coclite; cc. 30r-31r, Muzio Scevola; cc. 31, Coriolano; c. 31v, Cammillo e Porenno; cc. 32r-33r, Il Lamento di Torquato Tasso nell'Ospedale di S. Anna; cc. 33v-34r, La morte d'Ermengar$d a$; cc. 34v-35v, bozza di lettera a Sabatino e di un componimento poetico; cc. 36 , Discorsetto. Le ultime carte sono gravemente danneggiate. 
9. L'annegata di S. Baronto, carte mss., S. Baronto, 3 marzo 1903, 6 cc. Si parla del suicidio di una giovane di Lamporecchio, avvenuto l'8 febbraio 1903.

10. Epigrafe funebre dedicata ad Antonio Torrigiani, minuta ms., 20 novembre 1919, 1 c. La carta usata come supporto scrittorio è stratta dal n. 4 di «Nuove Pubblicazioni» (cfr. 2.1.13).

11. Non conobbe suo padre perché quando nacque era morto da quattro mesi..., minute mss., s.l., s.d., 4 cc. Bozza di scritto che parla di un bambino, Giacomino, nato in condizioni di disagio e povertà, e di sua madre, Caterina, di brutto aspetto e rimasta prematuramente vedova mentre era incinta del piccolo.

12. Fratelli Contadini, taccuino ms., s.l. [Lamporecchio], s.d. [18951914], $14 \mathrm{cc}$. Lungo discorso politico rivolto alle masse contadine.

13. IT a Renzo, minuta ms., Firenze, 20 novembre 1920,8 cc. IT redarguisce Renzo, protagonista di una violenta lite con la madre e reo di aver accusato lo stesso IT di averlo rimproverato troppo sul luogo di lavoro (calzoleria).

14. Tranquillo Gasperini [padre tutore di Renzo] a IT, lettera ms., Pistoia, 17 novembre 1920, 2 cc. Tranquillo Gasperini, padre di Renzo, prende le difese del figlio e respinge le accuse al mittente.

15. L'orfano del ricco e l'orfano del povero, carte mss., s.l., s.d., 2 cc. IT mette a confronto le conseguenze che ricadono sulle famiglie di due defunti padri, uno ricco, l'altro operaio.

16. La maggior colpa è del patrio governo che fra i problemi d'urgenza il meno che abbia pensato è stato alla pubblica istruzione..., carta ms., s.l., s.d. [1895-1914], 1 c. Discorso sull'importanza dell'istruzione come strumento d'emancipazione sociale della classe proletaria.

17. R. Corte d'Appello di Firenze (Sezione d'Accusa) - Memoria per Idalberto Targioni (parte civile) contro Fanti Telemaco (imputato), opuscolo, Pistoia, Tip. G. Flori, 1903, 16 pp. Contiene i «motivi a sostegno dell'opposizione della parte Civile IT contro l'ordinanza profferita dal Giudice Istruttore di Pistoia il 6 marzo 1903 nel processo penale contro Fanti Telemaco imputato di diffamazione».

18. Federazione Socialista di Casellina e Torri, Compagni Socialisti della Toscana!, opuscolo, Firenze, Tip. Polli, 1910, 4 pp. Si pubblicizza l'uscita di una nuova rivista diretta da IT, «Il Contadino Toscano. Periodico quindicinale del proletariato agricolo e di ogni categoria di operai braccianti diretto dall'ex contadino IT».

19. Voi siete la classe più benemerita dell'umanità e siete la piu disprezzata..., bozze mss., s.l. [Lamporecchio], s.d. [1895-1914], 2 cc. Bozza di discorso rivolto alle classi contadine che invita a non fidarsi ciecamente dei preti. 
20. Quando i nostri superuomini, filosofi orecchianti che ripetono le frasi fatte..., bozze mss., s.l. [Lamporecchio], s.d. [1895-1914], 2 cc. Bozza di discorso sul rapporto tra cervello e cuore.

21. Tempo è di respirare aure piu dolci amiche..., bozza ms., s.l., s.d. [18951914], 1 c. Bozza di poesia con versi anticlericali in rima baciata.

22. Due porzioni di bozza del dialogo tra Gaspero, filocattolico e difensore dell'operato dei preti, e Damiano, operaio socialista e anticlericale, minute mss., s.l. [Lamporecchio], s.d. [1895-1914], 2 cc.

23. Trascrizione di versi di Carlo Tresanini dedicati a Umberto I, Re d'Italia uscito incolume dall'attentato di Pietro Acciarito del 22 aprile 1897, carta ms., Firenze, 15 giugno 1897, 1 c.

24. Da quel giorno, Giulio e Silvia furono spesso insieme..., bozza ms., s.l., s.d., 1 c. Bozza del racconto della triste vicenda di due innamorati, Giulio e Silvia, che, impossibilitati a esprimere liberamente il loro amore, decidono di mettere fine ai loro giorni gettandosi insieme da un dirupo.

25. E qui una domanda è necessaria. Perché se tanto è aumentata la produzione per la maggioranza degli uomini non c'è nemmeno il pane quotidiano?..., minute mss., s.l. [Lamporecchio], s.d. [1895-1914], 2 cc. Bozza di discorso sulle sperequazioni presenti nella società capitalistica.

26. Contraddittorio monarchico e antimonarchico, carte mss., s.l., s.d. [1895-1914], 8 cc. Trascrizione di contraddittorio, composto da 55 strofe in ottava rima, tra Fortunato Chiti e IT, rispettivamente pro e contro l'istituzione monarchica.

27. Per l'inaugurazione della Bandiera socialista della Sambuca, taccuino ms., s.l., s.d. [1895-1914], 12 cc. Bozza preparatoria per un discorso da tenere in occasione dell' inaugurazione della bandiera della sezione socialista della Sambuca.

28. ...tempio di celesti armonie..., libretto ms., s.l. [Lamporecchio], s.d. [1895-1914], 10 cc. Bozza di scritto dedicato alla vita di Giuseppe Verdi.

29. Eterno sole che da piu secoli scaldi la terra..., bozza ms., s.l., s.d., 1 c. Bozza di componimento poetico dedicato al sol dell'avvenire che in futuro avrebbe finalmente scaldato un mondo di uomini uguali e liberi.

30. Contraddittorio tra un monarchico religioso e un socialista, carte mss., s.l. [Lamporecchio], s.d. [1895-1914], 8 cc. Contrasto in ottava rima tra Fortunato Chiti e IT su «Socialismo, Governo e Religione».

31. Gli antichi mitologici cantarono di Giove..., minute mss., s.l., s.d., 2 cc. Bozze di vari componimenti poetici.

32. Quaderno copialettere, bozze di componimenti poetici, discorsi e appunti vari, minute spillate e mss., s.l. [Lamporecchio], 1907, $68 \mathrm{cc}$. 
33. La suocera e la nuora. Dialogo farsa in un atto, carte legate mss., s.l., s.d., 6 cc.

34. Discorso sull' istruzione, foglio a protocollo ms., s.l. [Lamporecchio], s.d. [1895-1914], 2 cc. Elenco in sette punti di questioni da trattare sul tema dell' istruzione e successivo sviluppo delle argomentazioni.

35. Monologo, foglio a protocollo ms., s.l. [Lamporecchio], s.d. [1895-1914], $2 \mathrm{cc}$. Monologo sulla bontà del socialismo e sulle ingiustizie sociali.

36. Poesia sui diritti della donna, bozza ms., s.l. [Lamporecchio], s.d. [1895-1914], 1 c. Componimento poetico in cui si auspica l'eguaglianza dei diritti tra uomo e donna.

37. IT a Tranquillo Gasperini, minuta ms., Firenze, 19 novembre 192[?], $3 \mathrm{cc}$.

38. I Municipi nella Storia, bozza ms., s.l. [Lamporecchio], s.d. [18951914], 1 c. Ricostruzione storica dell' istituzione municipale a partire dai tempi degli etruschi e dei latini.

39. IT al direttore della «Rivista Popolare», carta ms., s.l. [Lamporecchio], s.d. [1895-1914], 1 c. L'autore ringrazia il destinatario per aver chiariti molti punti oscuri della questione sociale e per aver svelati tanti trucchi politici. Bozza incompleta.

40. Dopo le parole chiare e concettose dell'egregio compagno nostro, Salvadori..., foglio a protocollo ms., s.l. [Lamporecchio], s.d. [1895-1914], $2 \mathrm{cc}$. Trascrizione di un discorso politico tenuto sulla questione del dazio sul grano, di cui i socialisti sostenevano l'abolizione.

41. Sul disagio economico, bozza ms., s.l. [Lamporecchio], s.d. [18951914], 1 c. Bozza di discorso politico sulle cause del disagio economico e sulla battaglia intrapresa dai socialisti per eliminarlo.

42. 2uando tutti avremo la dovuta istruzione..., carta ms., s.l.[Lamporecchio], s.d. [1895-1914], 1 c. Discorso sull'importanza dell'istruzione quale elemento fondamentale per rendere il popolo cosciente dei propri diritti e dei propri doveri.

43. Ritaglio di pagine dedicate alla storia di Polduccio, carta ms., s.l., s.d., $1 \mathrm{c}$.

44. Ed ora, o contadini e operai tutti che mi ascoltate..., foglio a protocollo ms., s.l.[Lamporecchio], s.d. [1895-1914], 2 cc. Bozza di discorso politico sulle ingiustizie sociali.

45. Carme, foglio a protocollo ms., s.l. [Lamporecchio], s.d. [1895-1914], 2 cc. Componimento poetico molto probabilmente scritto da terzi (diversa grafia).

46. Taccuino con discorsi politici, minute spillate e mss., s.l. [Lamporecchio], s.d. [1895-1914], 12 cc. Bozze di discorsi politici.

47. I socialisti non negano che il cristianesimo..., carta ms., s.l., s.d. [18951914], 1 c. Bozza incompleta di discorso sul rapporto tra socialisti e la religione. 
48. Sulle origini delle Religioni e Come provano l'esistenza d'Iddio i credenti, carte mss., s.l. [Lamporecchio], s.d. [1895-1914], 5 cc.

49. Compagni di fede e compagni di lavoro!..., bozza ms., s.l. [Lamporecchio], s.d. [1895-1914], 1 c. IT denuncia che, nel corso di una sua conferenza, un delegato di P.S. sia intervenuto per togliergli la parola e per disperdere i convenuti.

50. Appunti, carta ms., s.l., s.d., 1 c.

51. Contraddittorio Pro e Contro la Donna, quaderno ms., s.l. [Lamporecchio], s.d. [1895-1914], 8 cc. Componimento poetico di 36 strofe in ottava rima dedicate alla figura della donna.

52. IT al Marchese [?], carte mss., Lamporecchio, 3 febbraio 1916, 2 cc. L'autore ringrazia il destinatario per avergli procurato un lavoro (venditore di calzature) che gli permettesse di vivere con più tranquillità.

53. Il mondo, chi bene lo riguardasse, somiglia in gran parte a una scala..., carta ms., s.l. [Lamporecchio], s.d. [1895-1914], 1 c. IT parla delle sperequazioni sociali esistenti nel mondo.

54. La fucilazione d'U. Bassi e I fratelli Attilio ed Emilio Bandiera, carte mss., s.l. [Lamporecchio], s.d. [1895-1914], 2 cc. Componimenti poetici dedicati ai martiri risorgimentali Ugo Bassi e i fratelli Bandiera.

55. IT al Direttore de «La Bandiera del Popolo di Pistoia», minuta ms., Lamporecchio, 6 ottobre 1920, $2 \mathrm{cc}$. IT tiene a controbattere quanto scritto da un articolista nel n. 36 del 1920 del suddetto giornale (cfr. 3.23.21).

56. Ferdinando Innocenti al Ministro del Tesoro, minuta ms., Lamporecchio, 9 dicembre 1922, 1 c. L'autore fa richiesta di un ulteriore sussidio per il mantenimento dei figli d'un suo figlio adottivo, Sparvieri Fernando, morto in guerra nel 1916.

57. Discorso sull'importanza dell' istruzione, bozza ms., s.l. [Lamporecchio], s.d. [1895-1914], 1 c. Bozza incompleta di discorso sull'importanza dell'istruzione presso le masse popolari e sull'ignoranza come origine delle disuguaglianze marcate presenti nella società borghese.

58. Le ricchezze, foglio a protocollo ms., s.l. [Lamporecchio], s.d. [18951914], 2 cc. Saggio sulla sperequazione delle ricchezze nel mondo.

59. Racconto sullo storpio Giacomino, bozza ms., s.l., s.d., 2 cc. Bozza incompleta.

60. IT al deputato [Napoleone Colajanni], minuta ms., Firenze, luglio 1920, 2 cc. IT ringrazia il Colajanni per averlo aiutato a riconquistare la libertà di pensiero. Parla poi della sua esperienza nel Partito Socialista, che critica aspramente, e dei motivi che lo hanno portato a sostenere, in un secondo momento, l'ingresso italiano nella guerra mondiale.

61. Appunti, carte spillate mss., s.l., 1920, 12 cc. Discorsi politici sull'anarchismo e sulla Repubblica dei Soviet in Russia. 
62. Un appello ai contadini della Toscana, fogli a protocollo spillati e mss., s.l., s.d. [1919?], 22 cc. Appello ai contadini sulle imminenti elezioni politiche del 19[19?].

8. Bambini Poveri (bozza 1), minute mss., s.l. [Firenze], s.d. [post 3 febbraio 1928], 4 cc. Componimento poetico in 20 strofe sul tema della povertà infantile. Le carte di supporto per la scrittura sono quattro copie di un documento della Confederazione Nazionale dei Sindacati Fascisti - Ufficio per la Provincia di Firenze datato 3 febbraio 1928.

9. Bambini Poveri (bozza 2), minute mss., s.l. [Firenze], s.d. [post 3 febbraio 1928], 2 cc. Scrittura più pulita rispetto alla bozza 1.

10. Bambini Poveri (bozza 3), carte dattiloscritte, s.l., s.d., 5 cc. Ultima revisione del componimento di cui sopra con 5 strofe in più.

11. Contadini, carte dattiloscritte, s.l. s.d. [1928], 20 cc. Bozza di componimento poetico dedicato al tema dei contadini, al sacrificio da essi compiuto nel conflitto mondiale e alle scarse gratificazioni da essi ricevute alla fine delle ostilità. Finale invocazione al Duce.

12. Cesare Battisti, minute dattiloscritte, s.l., s.d. [1928], 18 cc. Bozza di componimento poetico elegiaco dedicato a Cesare Battisti (1875-1916).

13. Il Mio cavallo, fogli a protocollo mss., s. l., s. d., $11 \mathrm{cc}$. Componimento poetico di 41 sestine con struttura ABABCC dedicato a un cavallo. Presenti 6 pp. di carte preparatorie e di bozza.

14. Canto agreste all'esimio chirurgo prof. Enrico Burci Rettore Magnifico dell'Università di Firenze, carte mss., s.l., s.d. [agosto 1926], 9 cc. Componimento poetico di 47 quartine elegiache dedicate al Magnifico Rettore dell'Università di Firenze, il prof. Enrico Burci.

15. Bacco Vol. I, quaderno ms., s.l., s.d., 40 cc. Componimento poetico in sestine con struttura ABABCC e versi liberi, diviso in tre parti e dedicato a «Il primo viaggio di Bacco in Toscana e i Brigidini di Lamporecchio», come recita il titolo presente a p. 1.

16. Bacco Vol. II, quaderno ms., s.l., s.d., 30 cc. Continuazione di Bacco Vol. $I$, con sestine con struttura ABABCC. Continuazione della parte terza e parte quarta.

17. I Brigidini di Lamporecchio, quaderno ms., s.l., s.d., 36 cc. Componimento poetico in 35 sestine con struttura ABABCC dedicate ai Brigidini, dolci di croccante sfoglia tipici di Lamporecchio.

18. Cartellina rossa recante la seguente scritta con inchiostro nero: «Bacco». Contiene i seguenti documenti:

1. O de' miei padri sacra itala terra..., carta ms., s.l., s.d., 1 c. Bozza di componimento poetico ms. di 10 quartine dedicate all'Italia.

2. Appunti sulla storia degli antichi romani, carte mss., s.l. [Firenze], s.d. [post 16 giugno 1924], 2 cc. Le carte di supporto sono di un documento a stampa del Consiglio Provinciale di Firenze del 16 giugno 1924. 
3. Contadini (bozza), carte mss., s.l. [Firenze], s.d. [1928], 6 cc. Bozza del componimento poetico dedicato ai contadini.

4. Carte preparatorie per Bacco e il Brigidino di Lamporecchio, carte mss., s.l., s.d., 6 cc. Su c. 2v, appunti abbozzati per discorso da fare a una pubblica adunanza fascista. A p. 12, documento del Comune di Lamporecchio, datato 26 settembre 1916, in cui il Sindaco IT dichiara di aver ricevuto dal Magazzino militare 62 paia di scarpe per conto del fornitore militare Alfredo Fanti.

19. Quaderno con pagine a quadretti intonse e con copertina celestina con scritta «POESIE» e contenente quanto segue:

1. Contemplando la mia Idalbertina a 22 mesi mentre dorme, carta ms., s.l. [Firenze], s.d. [presente graffetta con pezzetto di carta con annotazione a lapis scritta da una terza persona e postuma al componimento: «agosto 1916 Idalberta figlia avuta da Sofia Bacarelli»], $1 \mathrm{c}$.

2. Sulla Religione, carte mss., s.l., s.d., 3 cc. Trattazione in versi sul tema della religione intesa dall'autore come il frutto della superstizione e dell' ignoranza popolare.

3. Vorrei, carta ms., s.l., s.d., 1 c. Poesia composta da tre quartine con struttura ABAB.

4. Casa di Marcone, carta ms., s.l., s.d., 1 c. Poesia composta da tre quartine e due terzine dedicata a Casamarconi, paese situato sulle montagne pistoiesi. Sul verso è presente la bozza del componimento.

5. Il bolscevico evoluto..., carte dattiloscritte, s.l., s.d. [1920-1930], 2 cc. Poesia composta da cinque sestine e una quartina e dedicata dispregiativamente ai comunisti.

6. Ai morti e ai feriti del Carso, carte dattiloscritte, s.l., s.d., 2 cc. Poesia composta da sei sestine e dedicata ai soldati italiani Caduti o feriti nel primo conflitto mondiale.

7. Enrico Toti, carte dattiloscritte, s.l., s.d., 13 cc. Componimento poetico in terzine dedicato al martire di guerra Enrico Toti (1882-1916).

8. Il volto della nuova Italia, carte dattiloscritte, s.l., s.d., 8 cc. Componimento poetico con 34 quartine dedicate all'Italia e alle sue bellezze naturalistiche.

9. 5 Maggio 1921. Canto alla Nuova Italia, carte dattiloscritte, s.l., s.d., 5 cc. Componimento poetico composto da 10 ottavine dedicate all'esaltazione della Madre Patria Italia.

10. Timidetta, impaziente, ecco la bella..., carta datt., s.l., s.d., 1 c. Componimento poetico composto da 5 ottavine dedicate al tema del matrimonio. Correzioni dell'autore con inchiostro nero.

11. Pagine di libro con poesie, carte stampate, s.l., s.d., 2 cc. Pagine estratte da un libro con tre componimenti poetici: Per chi non sono e per chi sono i miei versi, La Spia, Ad un frate zoccolante. 
12. Brindisi per l'annuale fraterno banchetto al Bastione della Sandraccia la sera del 10 novembre 1912, Vigilia di S. Martino, carta stampata, s.l., 10 novembre 1912, 1 c. Sul verso è presente un breve elenco di spese.

13. Spartaco, carta ms., s.l., s.d., 3 cc. Componimento poetico composto da 13 quartine e una sestina dedicate a Spartaco.

14. Seduto al desco, fra le palme stanche..., carta datt., Lamporecchio, s.d. [1920-1930], 2 cc. Componimento poetico di 13 quartine dedicato al tema della vecchiaia.

15. Enrico Toti (bozza 2), carta ms., s.l., s.d., 1 c. (cfr. 2.23.7).

16. Componimento sulla guerra mondiale, bozza ms., s.l., s.d., $1 \mathrm{c}$.

17. Appunto sulla storia degli antichi romani, carta ms., s.l., s.d., 1 c.

18. Nanni [IT] a [destinatario non presente], minuta ms., s.l., s.d., 1 c. L'autore avverte il destinatario della sua ridiscesa in città e che spera di fargli pervenire quanto prima i versi che gli aveva promesso.

19. Dedicato al Sig. Otello Fedi. Per una promessa mancata (bozza 1), carta ms., s.l., s.d., 1 c. Componimento poetico composto da 7 sestine scritte da Fortunato Chiti.

20. Dedicato al Sig. Otello Fedi. Per una promessa mancata (bozza 2), carta datt., s.l., s.d., 1 c.

21. Trieste, carta datt., s.l., s.d., 1 c. Trascrizione di un sonetto recitato dinanzi ai lavoratori di Trieste l'8 gennaio 1912.

22. Il Piccolo Balilla e Giovanni Uss sul rogo, Ad un amico, Carducci e Rapisardi, carte dattiloscritte, s.l., s.d., 2 cc. Trascrizione di poesie, alcune recitate pubblicamente in vari luoghi: Ad un amico, Cosenza, 3 luglio 1912; Giovanni Uss sul rogo, Kruezlingen, 25 agosto 1912.

23. Il Piccolo Balilla, opuscolo ms., s.l., s.d., 7 cc. Componimento poetico dedicato ai piccoli Balilla pronti, con l'impegno, lo studio e il lavoro a servire fieramente la loro patria.

24. Vedevo Garibaldi galoppante..., foglio a protocollo ms., s.l., s.d., 2 cc. Bozza di componimento poetico dedicato a Garibaldi.

25. La Quercia (bozza), carta ms., s.l., s.d., 1 c.

26. La Lodoletta, carte mss., s.l., s.d., 2 cc. Componimento poetico composto da 14 quartine e dedicato a una lodola, allegoria della coscienza dell'umanità.

27. Gennaio (bozza), carta ms., s.l., s.d., 1 c.

28. Al Sig. Gaetano Ciappi per la sua magnifica acqua di colonia, carta ms., s.l., s.d., 6 cc.

29. Soliloqui di Montagna dedicato al mio fratello di sangue Bartolomeo Gavazzi, fogli a protocollo mss., s.l., s.d., 4 cc. Componimento poetico composto da 32 quartine e dedicato Bartolomeo Gavazzi, figlio dei primi genitori affidatari di IT.

20. Quadernino dalla copertina nera contenente i seguenti documenti: 
1. Appunti e note, taccuino ms., s.l. [Firenze], s.d. [1921], 30 cc. Raccolta di pensieri sulla bontà del fascismo della prima ora e di critica nei confronti degli ex compagni socialisti. I titoli posti dall'autore alle varie argomentazioni sono i seguenti: Seminatori d'odio, Fatti e non chiacchere, Diffamazione socialista, Il Fascismo antiscioperista, L'Idealismo fascista, I meriti del Fascismo, Confessiamo i nostri torti, La parte caduca del Socialismo, Il Programma fascista, Un ricordo, I fascisti non sono contro gli operai.

2. Bozza di componimento dedicato all'Italia, foglio a protocollo ms., s.l., s.d., 2 cc.

3. Bozza de I Brigidini di Lamporecchio, minute mss., s.l. s.d., 2 cc. (cfr. 3.17).

4. Preambolo - Raccolta di poesie, carte dattiloscritte e mss., s.l. [Firenze], s.d. [1921-1930], 32 cc. Alle pp. 1-18, Preambolo lirico. A p. 19, Invocazione alla Musa ispiratrice. Alle pp. 21-24, La Diana, che preannuncia l'arrivo di un gran messia [Mussolini?]. Alle pp. 25-26, Vigliacchetti, che accusa i fascisti dell'ultima ora, ovvero gli opportunisti. Alle pp. 27-28, Farisei, in cui l'autore si scaglia contro chi vorrebbe obbligarlo a star zitto. Alle pp. 29-40, La dottrinella fascista, in cui scrive in versi le regole del buon fascista. Alle pp. 41-50, Visioni, in cui si parla di soldati morenti al fronte che hanno una visione: un futuro di pace e lavoro.

21. Documenti diversi - Cartellina verde contenente i seguenti elementi:

1. A sua Eccellenza la Principessa Maria Demidoff per la morte immatura dell'amatissimo sposo Principe Simone Abamelech. Un servo oscuro e devoto offre, carte stampate, Firenze, 24 dicembre 1916, 2 cc.

2. Lavoratori Agricoli, carte dattiloscritte, s.l. [Firenze], s.d. [1929], 13 cc. Dedicato all' importanza e ai diritti dei braccianti.

3. «L'Avvenire», Pistoia, 24 ottobre 1908, 2 pp. A p. 2 è presente una poesia di IT, Fine d'Ottobre.

4. Libro e Rivoltella, foglio a protocollo ms., s.l. [Firenze], s.d. [19231930], 2 cc. Apologia in versi (quartine) del motto mussoliniano «Libro e moschetto, Balilla perfetto».

5. Enrico Toti (bozza 3), foglio a protocollo ms., s.l., s.d., 2 cc. (cfr. 3.19.7).

6. Idalberto Targioni. Educatore e organizzatore delle nostre masse rurali e contadine, carte sciolte mss., s.l. [Firenze], s.d. [1923], 8 cc. IT parla in terza persona della propria biografia, della sua attività di poeta estemporaneo e avanza critiche nei confronti del Partito Socialista di cui lui ha fatto parte.

7. Poesie varie, minute sciolte mss., s.l. [Firenze], s.d. [1923-1930], 3 cc. Bozze di componimenti poetici parziali: uno (contrassegnato dal numero romano in matita rossa «XII») in cui accusa i proprietari 
terrieri; un secondo (pagina «XVI»), in cui parla della bellezza e del suo mutare nel corso della vita; il terzo (pagina «XVIII») in cui esalta l'operato di Mussolini per la bonifica agraria e ne difende la politica fiscale.

8. Voci d'odio di rabbia e di dispetto..., bozza ms., s.l., s.d. [1923-1930], $2 \mathrm{cc}$. Bozze di componimento poetico in quartine in cui si getta disprezzo sui traditori della Patria e si esalta il Duce.

9. Consiglio Provinciale di Firenze, foglio a protocollo a stampa, Firenze, 25 giugno 1923, 2 cc. Deliberazione del Consiglio Provinciale di Firenze circa la costituzione di quattro Commissioni di lavoro. A p. 3 il nome di IT compare nell'elenco dei 15 consiglieri nominati per la Commissione alle Opere Pubbliche.

10. Prefetto di Firenze a IT, lettera datt., Firenze, 3 gennaio 1923, 1 c. Il Prefetto invita nel suo ufficio IT a relazionare circa il suo operato quale Segretario dei Sindacati Nazionali per l'Agricoltura della Provincia di Firenze.

11. Foste obbedita o Giovinezza!, bozza ms., s.l., s.d., 1 c. Bozza di poesia incompleta.

12. Il Viandante (bozza), carte mss., s.l., s.d., 5 cc. piegate in due. Bozza di componimento poetico in quartine dedicato a un viandante che s'avventura nell'allegorico cammino della vita.

13. Preghiera e La nostra bambina, carte mss., s.l., s.d., 4 cc. Alle pp. 1 e 3, Preghiera, poesia in sestine in cui IT si rivolge a Dio chiedendo protezione per i propri cari. Alle pp. 5 e 7, La nostra bambina, poesia probabilmente dedicata a Idalberta, la figlia avuta dal rapporto amoroso con Sofia Bacarelli. Le carte usate come supporto per la scrittura sono 4 copie di una pagina del numero 4 di «Nuove Pubblicazioni» (cfr. 2.1.13).

14. Scherzo all'amico Petracchi Giuseppe, carta datt., s.l., s.d., 3 cc.

15. All'amico Felice Tofanelli. Sc[h]erzo, carte sciolte mss., s.l., s.d., 3 cc. Oltre all'amichevole sberleffo in versi dedicato all'amico Felice Tofanelli, sulle altre pagine vi sono appunti per vari componimenti.

16. Poesia contro il carovita, carta ms., s.l., s.d., $1 \mathrm{c}$.

17. Enrico Toti (bozza 4), carta ms., s.l., s.d., 1 c. (cfr. 2.23.7).

18. Introduzione, bozza ms., s.l., s.d. [post 1928], 1 c. Bozza di Preambolo (cfr. 3.20.4). La carta usata come supporto per la scrittura è un documento a stampa della Corporazione Nazionale dei Sindacati Fascisti della Provincia di Firenze che invita alla campagna di tesseramento dell'anno 1928.

19. Bozze di Bacco, minute mss., s.l. [Firenze], s.d. [1924-1930], 2 cc. Supporto cartaceo contenente documento a stampa intitolato $\mathrm{Ai} \mathrm{Fa}$ scisti della mia Zona e firmato da Umberto Odett Santini, espulso dal Partito Fascista. 
20. Sentenze, schizzi e note volanti, carte mss., $3 \mathrm{cc}$.

21. Visioni (bozza), foglio a protocollo ms., s.l., s.d., 2 cc. (cfr. 2.24.4).

22. Bozze di poesie, carta ms., s.l., s.d. [1928-1930], 1 c. Su una pagina bozza di componimento poetico che elogia l'opera svolta dal Capo di Governo, Mussolini. Sull'altra pagina, invece, v'è la bozza di una poesia che parla di una fanciulla di umili natali che invidia un'amica appartenente a una famiglia facoltosa.

23. Salve a te valoroso comandante..., carta ms., s.l., s.d., 1 c.

24. Bozza di poesia lirica, carta ms., s.l., s.d., $1 \mathrm{c}$.

25. Poesie dedicate a Enrico Burci, minute mss., s.l. [Firenze], s.d. [cfr. $3.24 .14], 8 \mathrm{cc}$. Bozza di componimenti poetici in quartine dedicati al prof. Enrico Burci, medico chirurgo dell'Ospedale di S.M. Novella che ebbe in cura IT e a cui il poeta è profondamente riconoscente.

26. Bozze di componimenti poetici vari scritti a matita e penna rossa, minute mss., s.l., s.d., 6 cc. Due bozze di poesia dedicata alla figlia Rita e due di poesia lirica.

27. Bozze di componimenti poetici, minute mss., s.l. [Firenze], s.d. [19 ottobre 1928], 1 c. A p. 1 bozza di poesia lirica che il poeta scrive in occasione del suo sessantesimo compleanno.

28. Bozza de $I l$ mio cavallo, bozza ms., s.l., s.d., 1 c. (cfr. 3.7.13).

29. Resistenza, carta ms., s.l. [Lamporecchio], s.d. [1916-1918], 1 c. Poesia in terzine in cui si esorta il popolo a resistere sui fronti di guerra come sul fronte interno all'attacco del nemico.

30. Contro il nemico prussiano, carte mss., Lamporecchio, s.d. [19161918], 2 cc. Poesia contro gli eserciti nemici dell'Italia sui fronti di guerra.

31. Bozza di poesia lirica, carte sciolte mss., s.l., s.d., 3 cc. Poesia dedicata ai luoghi e alle prime fasi della vita del poeta.

32. Appunti scritti a lapis, carte mss., s.l., s.d., 4 cc.

22. «Versi Sparsi»-Cartellina viola contenente i seguenti elementi:

1. I' Lavoro, carte mss., s.l. [Firenze], s.d. [1922-1930], 2 cc. A p. 1 è presente parte di un discorso sul fascismo. A p. 4, I' Lavoro, poesia composta da 8 quartine, in cui si esalta il lavoro svolto dagli avi nel costruire architettonicamente e non solo il nostro Paese. Il supporto cartaceo è ricavato dal retro di una copia del numero 4 di $\ll$ Nuove Pubblicazioni» (cfr. 2.1.13).

2. Viposso assicurar che in quel momento..., carta ms., s.l., s.d. [post 1912], 1 c. Sul recto è presente una poesia in quartine in cui IT parla delle antiche civiltà di Tebe e Babilonia. Il supporto cartaceo è ricavato dal retro di una copia del numero $4 \mathrm{di}$ «Nuove Pubblicazioni» (cfr. 2.1.13).

3. Ai contadini di S. S., manifesto a stampa, Firenze, s.d. [pre 1915], 2 pp. Componimento poetico anticlericale in ottava rima firmato «Rosignolo», uno dei soprannomi di IT. 
4. Bacco (bozza), fogli a protocollo e carte sciolte mss., s.l. [FirenzeLamporecchio], s.d., 13 cc. (cfr. 2.15-16).

5. Alla terra (carme), carte mss., s.l. [Firenze-Lamporecchio], s.d., 3 cc.

6. Cinquantesima Primavera, carta ms., s.l. [Firenze-Lamporecchio], s.d. [1918], 2 cc.

7. Appunti e bozza di poesia, carta ms., s.l. [Lamporecchio], s.d. [19141915], 1 c. Appunti di argomento politico e bozza di componimento poetico dedicato a una donna dal corpo cadente.

8. Ignoranza e Miseria; ecco $i$ due mostri..., fogli a protocollo mss., s.l. [Firenze-Lamporecchio], s.d. [probabilmente pre 1915], 6 cc. Componimento poetico in cui l'autore cerca d'istruire i proletari schiacciati dall' ignoranza e dalla miseria.

9. Battaglia di Gorizia, carte ms., Firenze, s.d. [post 1923], 2 cc. Il supporto cartaceo usato per la scrittura è una carta piegata in due con la seguente intestazione in maiuscolo: «Federazione dei Sindacati Fascisti della Provincia di Firenze/ Corporazione Agricoltura/ Segreteria Provinciale/ Lavoratori Agricoli/ Coloni e Braccianti».

10. Il mio cavallo dedicato a tutte le Signore di mia conoscenza, fogli a protocollo e carta mss., s.l. [Firenze-Lamporecchio], s.d., 15 cc. Bozze varie di componimento poetico dedicato a un cavallo.

11. Per le fauste nozze dell' amico Fausto Maccheroni colla gentil fanciulla Elisa Innocenti, carta datt., s.l. [Firenze], 30 luglio 1923, 1 c.

12. Fra i campi e gli orti del pian pratese..., carta ms., s.l. [Firenze-Lamporecchio], s.d., 1 c. Componimento poetico incompiuto d'invettiva contro tre figure criminali dell'area pratese.

13. Brindisi agli amici carissimi Alessandro Ponzia e Alberto Tommaso [...] offre Adolfo Poggi, foglio a protocollo ms., s.l. [Firenze], marzo 1929, 2 cc. Probabilmente scritto da IT per conto di Adolfo Poggi, o scritto da Poggi e corretto da IT.

14. IT al Prof. Antonio Comolli, copia di lettera ms., Vallombrosa, 24 agosto 1926, 1 c. L'autore informa il medico circa il suo stato di salute e che sta preparando un componimento elegiaco dedicato al Prof. Burci.

15. Bozza di Ai morti e ai feriti del Carso, carta ms., s.l., s.d., 1 c. (2.23.6).

16. Bozze di poesie, carte mss., s.l., [Firenze], s.d. [1923-1930], 2 cc. Componimento poetico in cui l'autore invita i lettori a non lamentarsi dell'attuale situazione politica. Le carte usate come supporto per la scrittura sono ricavate dal retro di due copie di un documento a stampa con la seguente intestazione: «Corporazioni Sindacali Italiane/ Sezione di Certaldo».

17. Bozze di poesie, carte mss., s.l. [Firenze], s.d. [1923-1930], 2 cc. IT esprime la sua impossibilità a contenere lo sdegno per canaglie e farabutti. Le carte usate come supporto per la scrittura sono ricavate 
dal retro di un documento a stampa con la seguente intestazione: «Corporazione Sindacati Fascisti/ Sezione di Marcialla».

18. [firma illeggibile] a IT, lettera ms., s.l. [Roma], 25 marzo 1916, 2 cc. Il mittente, probabilmente un deputato, promette di difendere IT in Consiglio di Stato e gli consiglia di non dimettersi dalla carica di Sindaco.

19. Poesia su Giolitti, carte sciolte mss., s.l., s.d., 5 cc. L'ultima carta presenta la bozza di una lettera indirizzata all'«Amico Del Rosso».

20. Approfittiamo di questo momento o compagni..., carta ms., s.l., s.d. [pre 1915], 2 cc. Componimenti diversi: a p. 1, scritto in orizzontale, e alle pp. 2-3, poesia contro la religione e il capitalismo; a p. 2, poesia dedicata a Emile Zola; alle pp. 3-4, poesia dedicata a un amico di nome Giulio.

21. IT al Direttore della «La Bandiera del Popolo» di Pistoia, minuta ms., Firenze, 7 ottobre 1924, 2 cc. IT informa il destinatario di aver querelato per ingiurie e diffamazione il giornale stesso e l'autore di un articolo pubblicato sul suo conto il 5 ottobre 1924 (cfr. 2.7.56 e 5.102).

22. Gli mosse contro con ira puntando... - Appunti, carta ms., s.l. [Firenze], s.d. [post 6 ottobre 1924], 1 c. La carta usata come supporto per la scrittura è ricavata dal retro di un documento a stampa, datato 6 ottobre 1924 e con la seguente intestazione maiuscola: «Consiglio Provinciale/ di/ Firenze».

23. Cartellina rossa contente articoli di giornale scritti da terze persone, scritti di e opere edite di IT. Gli elementi contenuti sono i seguenti:

1. Spinoza. Il $250^{\circ}$ dalla sua morte, ritaglio de «La Nazione», s.d. [1927], 1 c.

2. Niccolò De' Colli, Giovanni Ruskin e Giovanni Papini, ritaglio de «La Nazione della sera», Firenze, 18 settembre 1923, 1 c.

3. Raccolta di ritagli di giornale con articoli di IT, fogli a protocollo con ritagli di giornali incollati, $4 \mathrm{cc}$. Gli articoli sono i seguenti:

1. Non fu, o uomo, la disobbedienza al divino comando..., «L'Avvenire», Pistoia, s.d. [aprile 1906]. Articolo anticlericale.

2. Una mala "Pasqua"..., «La Difesa Religiosa», Pistoia, 21 aprile 1906. Articolo di risposta a quello precedente di IT.

3. La risposta d'un malato ai reverendi della "Difesa", «L'Avvenire», Pistoia, 4 maggio 1906. IT alimenta la polemica con «La Difesa».

4. Ad un "malato" dell'AVVENIRE socialista, «La Difesa Religiosa», Pistoia, s.d. [maggio 1906]. Contro-risposta del giornale filoclericale a IT. 
4. Raccolta di ritagli di giornale con poesie di IT, 2 cc.

I ritagli sono i seguenti:

1. Una favola moderna ovvero Il Consiglio de'topi, s.l., s.d. Componimento poetico di 18 strofe in ottava rima.

2. Dice che del santo di cui ho profanato le sacre reliquie... [senza titolo], S. Baronto, 13 agosto 1901. Articolo anticlericale in polemica con Padre Ferruccio Bianchi e Guido Lassi.

3. Il ragno, «L'Avvenire», Pistoia, s.d. [1901-1910].

4. Lettera aperta, Lamporecchio, 20 maggio 1902. Articolo pubblicato su [?] in polemica con Don Giuseppe Bondi, parroco di Petrazzi (fraz. di Castelfiorentino).

5. Ci rivolgiamo alla ben nota di Lei cortesia... [senza titolo], «Vita Nuova», Empoli, [dicembre 1902]. Si testimonia che IT ha subito ingiurie da un tale sig. Fanti di Montaione.

6. Maggio Maledetto, [«L'Avvenire»], [Pistoia], s.d. Poesia in quartine in cui l'autore auspica un futuro roseo per i lavoratori.

5. Ilparto dell'orso, carta stampata, Firenze, Tip. E. Ducci, 1907, 1 c. Componimento poetico anticlericale con 28 strofe in ottava rima.

6. Per la serata d'onore del $M$. direttore e Concertatore d'Orchestra Cav. Uff. Vittorio Bellini al R. Teatro Manzoni alcuni ammiratori offrono, s.l., Tip. Fiori, 20 febbraio 1906, 2 pp. Elegia in versi dedicata al direttore d'orchestra Vittorio Bellini.

24. Prefazione, carte sciolte mss., s.l. [Firenze], s.d. [1923-1930], 7 cc. Presente componimento in sestine dedicato ai contadini, ai lavoratori e volto a esaltare il Duce. Su c. 1v, discorso politico inneggiante la Patria e il Duce. Alle cc. 6v e 7r, appunti sulla religione. Alle cc. 6r e $7 \mathrm{v}$, saggio intitolato La Patria (cfr. 1.1.8).

25. Bacco-Appunti, carte mss., s.l., s.d., 4 cc. (cfr. 2.15-16).

26. La casa (bozza), foglio a protocollo ms., s.l., s.d. [1921-1930], 2 cc. (cfr. 2.1.6).

27. La Guerra Europea, carte mss., s.l. [Firenze], s.d. [1918-1930], 10 cc. Componimento poetico dedicato alla Grande Guerra cantata in termini patriottici. Come supporto scrittorio sono state usate cinque copie del numero 4 di «Nuove Pubblicazioni» (cfr. 2.1.13).

28. Per le fauste nozze della gentilissima Signorina Gina Nerozzi coll'esimio giovane Salvatore Paterniuti la cugina Amelia Poggiali offre, carte mss., s.l. [Firenze], 3 agosto 1924, 1 c. Il supporto di scrittura è ricavato dal retro di un documento a stampa con la seguente iscrizione: «Elenco delle deliberazioni prese per urgenza dalla Deputazione Provinciale». 
29. Canti americani. Il poeta descrive la Borghesia d'un villaggio presso $S$. Francisco, s.l. [Lamporecchio], s.d. [pre 1915], 6 cc. Poesia di critica alla borghesia di San Francisco negli USA.

30. Bozza di Canti americani, minute mss., s.l., s.d., 2 cc.

31. Bozza di poesia dedicata a Sofia Bacarelli, carte mss., s.l. [Firenze], s.d., 3 cc.

32. Bozza di poesia dedicata alla «vilipesa Plebe», carte mss., s.l., s.d., 5 cc.

33. La resurrezione dei morti nel cimitero di S. Baronto, fogli a protocollo mss., s.l. [Lamporecchio], s.d. [1905-1906], 11 cc.

34. Quanta penuria $v$ ' ha di buoni il mondo... [senza titolo], fogli a protocollofogli a protocollo mss., s.l. [Lamporecchio], s.d. [pre 1915], $4 \mathrm{cc}$. Bozza di componimento poetico in cui si parla delle disparità sociali.

35. Trascrizione di confronto in ottava rima tra IT e Chiti su «Socialismo, Governo e Religione», fogli a protocollo mss., s.l. [Lamporecchio], s.d. [pre 1915], 10 cc. (cfr. 2.7.31).

36. Contraddittorio Chiti-Targioni sulla Guerra Libica, carte mss., s.l. [Lamporecchio], s.d. [pre 1915], 8 cc.

37. Ferdinando Chini, La mia vita militare. In ottava rima, carte dattiloscritte, s.l., s.d., 4 cc.

38. C. B., Qual novel padre Zappola..., carta ms., Larciano, 3 aprile 1921, 1 c. Poesia polemica rivolta all'amministrazione di Larciano e volta ad esaltare i fascisti.

39. Bozza di poesia dedicata a Giovanni Berta e al Duce, carte mss., s.l. [Firenze], s.d. [1921-1930], 2 cc.

40. La Lodoletta (bozza), minute mss., s.l., s.d., 2 cc. Bozza di componimento poetico in quartine dedicato a una lodola che si rivolge ai lavoratori.

41. Rita (bozza), carta ms., s.l. [Lamporecchio], s.d., 1 c. (cfr. 3.23.26).

42. L'Inno dei braccianti, carta ms., s.l., s.d. [1915-1930], 1 c. Bozza di componimento poetico di cinque sestine dedicato ai braccianti agricoli.

43. Gran Dio!, carte mss., s.l., s.d. [1920-1930], 2 cc. Supplica a Dio in prosa.

44. Scritto politico esaltante Duce e Corporazioni, carta ms., s.l. [Firenze], s.d. [1923-1930], 1 c.

45. Riguardo un'adunanza sull'organizzazione dei Sindacati Agricoli, bozza ms., s.l. [Firenze], s.d. [1923-1930], 2 cc. Sul retro, bozza di poesia, Il viandante.

46. Rapidi cenni della mia opera Fascista dal 1916 al 1929, carte mss., s.l. [Firenze], s.d. [1929-1930], 6 cc. L'autore parla in terza persona della sua carriera politica dalle fila socialista a quelle fasciste. 
47. Ill.mo Sig. Professor Bonfatti Commissario Straord. Dei Sindacati Fascisti di Firenze dedico a Lei questi rapidi cenni biografici [...] sulla mia opera socialista e di Fascista, bozze mss., s.l. [Firenze], s.d. [19291930], 7 cc. L'autore parla in terza persona della sua carriera politica dalle fila socialista a quelle fasciste.

48. Bozza di lettera indirizzata a fascista fiorentino, minuta ms., Firenze, s.d. [1924-1925], 1 c. L'autore denuncia, senza farne il nome, due massoni che si sono infiltrati negli ambienti del Fascismo.

49. Il Bastardo, fogli a protocollo mss., s.l., s.d. [pre 1915], 10 cc. Dialogo tra Nerina e Carlo Alberti sulla festa dei lavoratori.

50. Ed oltre l'Equatore sfrutteranno Washington e Franklin, carta ms., s.l., s.d. [1916-1930], 1 c. Componimento poetico in cui l'autore esalta la madre Patria.

51. Appunti. Note, dati statistici, estratti 1926, fascicolo ms., s.l. [Firenze], $1926,10 \mathrm{cc}$.

52. Il volto della nuova Italia (bozza), minute mss., s.l. [Firenze], s.d. [1923-1930], 7 cc. (cfr. 3.19.8).

53. Bozza di poesia all'Italia e al Duce, minute mss., s.l. [Firenze], s.d. [1923-1930], 5 cc. (cfr. 3.23.22).

54. IT a Sofia Bacarelli, minuta ms., Pistoia, 6 febbraio 1922, 2 cc. IT informa Sofia delle sue attività politiche nella zona di Pistoia.

55. Io e la mia anima, Cos'è la vita, Sulla Montagna - Raccolta di poesie, carte dattiloscritte, s.l. [Firenze], s.d. [1923-1930], 6 cc. Raccolta di componimenti poetici: c. 1, Io e la mia anima; cc. 2r-3v, Cos'è la vita; cc. 4r-6v, Sulla montagna. Su c. 1r, presenti due timbri con la seguente iscrizione in maiuscolo: «Il Segretario Provinciale dei Sindacati Coloni e Braccianti».

56. Poesia ad Agostino Nasti, carte dattiloscritte, Firenze, 30 giugno 1928,3 cc. Poesia in versi martelliani dedicati al giovane fascista Agostino Nasti. Carte intestate con la seguente iscrizione: «Confederazione dei Sindacati Fascisti/ Ufficio per la Provincia di Firenze».

57. Voi direte che son matto..., carte mss., s.l., s.d., 2 cc. Bozza di poesia in cui l'autore si pone domande esistenziali. Su c. 2 v, sul retro della carta di più piccole dimensioni, «Dati Statistici», concernenti tasso di natalità e mortalità dell'Italia.

58. Seduto al desco fra le palme stanche..., s.l., s.d., 2 cc. Poesia lirica in quartine dedicate al passare del tempo e all'esaurirsi della vita.

59. Per le fauste nozze della Gentil Signorina Enrica Baccioni col distinto Dottore Goffredo Pieri in segno di puro affetto e di vera amicizia ai giovani sposi Enrico Raspanti offre, carte dattiloscritte, s.l., s.d., 2 cc. Scritto probabilmente da IT per conto di Enrico Raspanti.

60. Brindisi, bozze mss., s.l. [Firenze], s.d. [1921-1930], 4 cc. Bozza di componimento poetico goliardico dedicato alla gioventù fascista. 
61. La fidanzata del Pittore, carta ms., s.l., s.d., 1 c. Sonetto dedicato a un pittore elogiato dalla fidanzata.

62. Battaglia Muliebre, carta ms., s.l., s.d., 1 c. Componimento poetico dedicato allo scontro verbale tra marito e moglie.

\section{Scatola 3}

Consistenza: 34 docc., 13 op. a st., di cui 6 periodici; 1.128 cc., 170 pp.

La scatola contiene prevalentemente quaderni e taccuini con appunti vari, bozze di prose, poesie e di discorsi politici di IT. Presenti anche pergamene a stampa con poesie dedicatorie a Laura T. Merrick o con altro contenuto.

1. All'Egregio Sig. Targioni Idalberto eletto con plebiscito unanime Consigliere Comunale di Lamporecchio, pergamena a stampa, Lamporecchio, 1926, 1 c. Attestato di conferimento della carica di Consigliere Comunale di Lamporecchio a IT.

2. Alla Nobile Signora Laura T. Merrick pel suo fausto ritorno alla Villa di Papiano le bambine Velia e Rita Targioni in segno di perenne affetto offrono, pergamena a stampa, s.l. [Lamporecchio], maggio 1902, 1 c.

3. Alla Nobile Signora Laura T. Merrick pel suo fausto ritorno alla Villa di Papiano le bambine Velia e Rita Targioni offrono, pergamena a stampa, Pistoia, Casa Tipo-Lito Edit. Sinibuldiana G. Flori \& C., 3 giugno 1903, 1 c., 2 copie.

4. Rita e Velia Targioni alla loro amata e gentil Signora Laura T. Merrickpel suo fausto ritorno alla Villa di Papiano, pergamena a stampa, s.l. [Lamporecchio], maggio 1904, 1 c.

5. Alla Nobile e Benemerita Signora Laura T. Merrick pel suo fausto ritorno alla Villa di Papiano le bambine Velia e Rita Targioni in segno d'affetto e devozione perenni offrono, pergamena a stampa, Empoli, Tip. Traversari, giugno 1905, $2 \mathrm{cc}$.

6. Alla Nobile e Gentilissima Signora Laura T. Merrick pel suo fausto ritorno alla Villa di Papiano la giovinetta Velia Targioni in segno d'affetto e di gratitudine perenni offre, pergamena a stampa, s.l. [Lamporecchio], maggio 1906, 1 c.

7. Fascio di Lamporecchio - Sezione di S. Baronto, poster fotografico, Lamporecchio, 1925, 1 c. Sono raffigurati Mussolini e i quadrumviri del Fascismo, 53 membri del Fascio di Lamporecchio, 29 membri del Fascio di S. Baronto.

8. «Il Segno di Empoli», rivista, anno 6, n. 21, aprile 1993, 32 pp. A pp. 8-9, articolo di Luisa Martini intitolato In memoria di Idalberto Targioni uomo prima tanto amato, poi, tanto vituperato ed, infine, troppo presto dimenticato.

9. Scherzo comico ovvero un contraddittorio tra un Prete democratico cristiano e un operaio meccanico socialista, quaderno ms., s.l. [Lamporecchio], 
s.d. [pre 1914], $26 \mathrm{cc}$. Quaderno con copertina rossa raffigurante un cameo nero con testa di nobildonna. Sull'interno di copertina, alla fine del quaderno, è presente la bozza ms. della poesia dedicata a Gabriele D’Annunzio.

10. Quaderno con copertina de «La Giovine Italia. Rivista di combattimento sociale politico letterario», Lamporecchio, 1909, $22 \mathrm{cc}$. Bozza di scritto poetico su Emile Zola sulle cc. 2r-3v; sulle cc. 4r-15r, bozza di Statuto di una Società Cooperativa tra braccianti e lavoratori della terra di San Baronto, presieduta da IT; sulle cc. 15v-16v, bozza di scritto burocratico attinente l'attività di Fortunato Chiti in qualità di tintore di trecce.

11. Io mi ricordo sempre una favoletta che mi raccontava la mia povera mamma, carta ms., s.l., s.d., 1 c. Sul recto, favola dei topi. Sul verso è presente la bozza di un discorso politico fatto in presenza di operai.

12. Quadernino con copertina azzurra con bozze di due scritti, quaderno ms., s.l., s.d. [pre 1915], 6 cc. sulle cc. 1r-2v, bozza di scritto sulle ricchezze naturali che compongono il mondo; sulle cc. $3 \mathrm{r}-6 \mathrm{v}$, bozza del secondo discorso pronunciato da IT in presenza di operai pistoiesi.

13. Taccuino con bozze di componimenti poetici, discorsi e appunti vari, taccuino ms. (a lapis), s.l., s.d. [pre 1915], 29 cc.

14. La prima venuta di Bacco in Toscana e i Brigidini di Lamporecchio. Poema Eroicomico dell'estemporaneo autodidatta Idalberto Targioni, quaderno ms., s.l. [Firenze-Lamporecchio], 1924, solo prime 10 cc. (cfr. 2.15-17).

15. Conferenze in versi di Idalberto Targioni, taccuino ms., s.l. [Lamporecchio], 1905, $45 \mathrm{cc}$. Appunti vari e trascrizione in versi di conferenze tenute presso la Camera del Lavoro di Firenze e la Società Giuseppe Verdi di Prato.

16. Manoscritti, taccuino ms., s.l., s.d. [1902-1914], 96 cc. Appunti di appuntamenti ed elenchi di spese, bozze di scritti politici e di componimenti poetici.

17. Taccuino marrone «Cartoleria Rosa Giannini Empoli», taccuino ms., s.l. [Lamporecchio], s.d. [pre 1914], 39 cc. Bozza di componimento poetico dedicato a La donna nel sistema borghese, seguito dalla bozza di un altro discorso in versi incentrato sulla giustizia sociale. Sulle cc. 34r-38r, bozza di discorso politico rivolto ai cittadini. Sull'interno della copertina e sulle ultime 3 pp. presenti appunti vari scritti a lapis.

18. Motti e note volanti, quadernino ms., s.l. [Lamporecchio], s.d. [19031904], 33 cc. Appunti vari, dati statistici, discorsi politici, elenchi di spesa, nomi e indirizzi di persone.

19. Quadernino senza copertina e senza titolo, quadernino ms., s.l. [Lamporecchio], s.d. [1900-1901], 59 cc. Appunti vari, nomi e indirizzi di persone, bozze di componimenti poetici e di discorsi politici, alcuni dei quali concernenti la guerra dei boxers in Cina. 
20. Taccuino con raccolta di componimenti poetici, taccuino ms., s.l. [Lamporecchio], s.d. [1900-1901], $59 \mathrm{cc}$ I titoli delle opere poetiche qui raccolte sono i seguenti: A Giuseppe Mazzini, A Francesco Benelli, Per l'Arte - L'avvenire, Sulla tomba di G. Guidi, Sul Rogo, Congedo, Saluto, Vale, Definizione, L'Eroe, Ferruccio, Il Progresso, L'utile del poeta, I poeti da cantina, La sera, Maffia, Camorra e Borghesia, Sul Caffè Sciantà, Su Attilio Frosini, Il coatto Politico, Per l'amnistia, A Dio, Sempre Avanti, Fin de Sicle, L'orfanella mendicante, Secolo vecchio secolo novo, Morta, Morta in bara, Terzine, Genesi della Creazione, La Donna, Sul Rogo, Gli ultimi istanti di Monti e Tognetti, Agli elettori incoscienti, Confronto, Ai tiranni della Libertà, Spartaco, Sposa moribonda, Fiori.

21. Taccuino con appunti, elenco spese e appuntamenti, s.l. [Lamporecchio], s.d. [1918], 32 cc. Presenti appunti di vario tipo circa appuntamenti, fabbisogno di scarpe e vestiario per popolazione e soldati, calcoli matematici.

22. Taccuino con elenco spese, orario treni, appuntamenti, appunti su viaggio in Svizzera e altro, s.l., s.d. [1908?], 18 cc. Elenco spese, orario treni, appuntamenti e appunti su un viaggio in Svizzera. Presente anche un piccolo vocabolario e prontuario con frasi di comunicazione basilare tradotte in tedesco.

23. Taccuino con discorsi politici, minute mss., elenco spese e nominativi, appunti vari, s.l. [Lamporecchio], s.d. [1902], 56 cc. Nelle ultime pagine, in senso capovolto, discorso di presentazione de Il Canzoniere. Poesie Sociali (1902).

24. Note satiriche morali - Note sociali - Note politiche, taccuino ms., s.l. [Lamporecchio], 1907, $38 \mathrm{cc}$.

25. Taccuino con scritti dal tema politico-sociale, taccuino ms., s.l. [Lamporecchio], s.d. [pre 1914], $40 \mathrm{cc}$. I titoli dei suddetti scritti sono i seguenti: Noi siamo api, La favola della quercia, Cosa ci fanno, Dolori occulti, Il socialismo collettivista.

26. Canto Primo - Era nel cuor d'estate e le chiesuole..., taccuino ms., s.l. [Lamporecchio], s.d. [pre 1914], 92 cc. Poema in ottava rima diviso in sei canti concernente una storia d'amore che ha luogo sul Montalbano e dal taglio anticlericale.

27. Bozzetti Sociali in versi, taccuino ms., s.l. [Lamporecchio], s.d. [1912], $136 \mathrm{cc}$. Ventotto componimenti poetici, molti dei quali convogliati poi nel Canzoniere di poesie sociali.

28. Taccuino con articoli di giornale di IT, taccuino con ritagli di giornale incollati, s.l. [Lamporecchio], s.d. [1904-1905], 46 cc. Si presume che gli articoli in questione siano stati prevalentemente estratti da «Avvenire», giornale socialista pistoiese. Gli articoli riportati sono i seguenti: Per la coltivazione della vite - Vincenzo Guazzini I-IV, Contro i congregazionisti, Poco dicono e nulla fanno, Amsterdam, Ai nostri morti, Le dimissioni del candidato Targioni (Lamporecchio, 22-10-1904), Carnevale, Il trasporto 
di Telemaco Boccaccini, Enrico Ferri, Per le due tendenze, Primo Maggio!, La Guerra, Reazione giolittiana, I veri miserabili.

29. Quadernino con bella copia di conferenze in versi, quaderno ms., s.l. [Lamporecchio], s.d. [pre 1914], $35 \mathrm{cc}$. Trascrizione di tre conferenze in ottava rima sui seguenti temi: Come fu prodotta la ricchezza sociale e le vittime del sistema borghese, Per la civiltà della Donna, La politica attuale.

30. Figure d'eroi e ultimi canti, quaderno ms., s.l. [Firenze-Lamporecchio], 1925, 30 cc. Componimenti poetici dedicati agli eroi e ai Caduti della Prima Guerra mondiale, alla madre patria Italia e al fascismo. Le poesie sono le seguenti: La battaglia di Gorizia, Cesare Battisti, Ai morti e ai feriti del Carso, Il volto della Nuova Italia, Ai villeggianti del Grappa.

31. Cinquant anni della mia vita (ricordi e memorie), libro datt., Lamporecchio, 1920, $104 \mathrm{cc}$. Autobiografia incompiuta e mai data alle stampe di IT.

32. «La Vita - Pistoia», Pistoia, 21 agosto 1966, 2 pp. Articolo di Carlo Migliorati intitolato Un poeta agitatore popolare. Idalberto Targioni (cfr. 1.18).

33. Carlo Migliorati, Lettere al Direttore. Ancora di Idalberto Targioni, ritaglio di giornale, «La Vita - Pistoia», Pistoia, 4 settembre 1966, 1 c. Lettera inviata da Lida Chiappini per correggere quanto riportato sull'articolo del direttore Migliorati in merito alle vicende biografiche di IT.

34. «La Blouse. Rivista Sociale», rivista a stampa, Anno 1, N. 5, Camera del Lavoro di Firenze, agosto 1906, 16 pp.

35. IT a Velia, lettera ms., Firenze, 2 febbraio 1927, 2 cc. IT informa la figlia circa il suo stato di salute fisica e morale e su una sua prossima visita a Empoli per questioni sindacali.

36. Socialismo e Anticlericalismo e bozze varie, carte spillate, s.l. [Lamporecchio], s.d. [pre 1915], 15 cc.

37. Elenco spese ed entrate e bozza di componimento poetico sul Montalbano, carte ms., Lamporecchio, s.d., 4 cc.

38. G. E. Modigliani, Per le prossime elezioni generali politiche. X Congresso Nazionale del Partito Socialista Italiano (Firenze, 19-20-21-22 Settembre 1908), opuscolo, Roma, Tip. Popolare, 1908, 8 pp.

39. I socialisti e il processo Murri, opuscolo, Firenze, Tip. E. Ducci, 1905, 16 pp.

40. La crisi della alimentazione in Germania ed in Austria, opuscolo, Ufficio di propaganda per la disciplina dei consumi presso il Ministero dell'Agricoltura, Roma, Off. Poligrafica Italiana, 1917, 32 pp.

41. «Il Risveglio della plebe», S. Baronto, 23 luglio 1908, 4 pp. A p. 1, articolo di IT: Francesco Minghetti; a p. 2, Combattiamo il prete.

42. «Il Risveglio della plebe», S. Baronto, [gennaio] 1909, 4 pp. Alle pp. 1-2, articolo di IT: 1909. A p. 3, componimento poetico: La suonata del Piovano. 
43. «L'Avvenire», Pistoia, 1 maggio 1911, 4 pp. A p. 1, articolo di Targioni: $1^{\circ}$ maggio. Pasqua proletaria; a p. $2,1^{\circ}$ Maggio Pistoiese.

44. «Giovinezza. Settimanale di fede e pensiero fascista», Empoli, 19 febbraio 1922,4 pp.

45. R. Corte d'Appello di Firenze (Sezione d'Accusa) - Memoria per Idalberto Targioni (parte civile) contro Fanti Telemaco (imputato), opuscolo, Pistoia, Tip. G. Flori, 1903, 16 pp., 2 copie (cfr. 2.7.18).

46. IV Sezione del Consiglio di Stato - Controricorso Comune di Lamporecchio intimato contro Fiorineschi Dr. Michele ricorrente, opuscolo, Pistoia, Tip Cino dei Fratelli Bracali, 1916, 20 pp., 2 copie.

47. Garibaldi a Pistoia, opera a stampa, Pistoia, 17 luglio 1904, 12 pp.

\section{Scatola 4}

Consistenza: 568 docc., 13 op. a st., di cui 4 periodici; 962 cc., 98 pp.

Contiene prevalentemente la corrispondenza personale di IT e documenti inerenti le attività del Fascio di Lamporecchio, di cui IT è stato Segretario tra il 1920 e il 1922.

1. Busta trasparente con corrispondenza familiare. Contiene i seguenti elementi:

1. IT al Tenente di Picchetto - Caserma Cavalleggeri, cartolina ms., Firenze, 23 agosto 1916, 1 c. IT chiede di concedere alla figlia Velia il permesso di visitare il marito Torello Chiappini, ricoverato in quella infermeria militare.

2. IT a Velia, cartolina ms., s.l. [Firenze], 12 aprile 1917, 1 c. IT informa la figlia circa le condizioni di salute di Torello.

3. IT ad Amelia, cartolina ms., s.l. [Firenze], 3 dicembre 1917, 1 c. IT informa la moglie di essere stato due giorni a Roma e di essersi strapazzato.

4. IT a Velia, lettera ms. + busta, s.l., 26 dicembre 1917, 1 c. IT informa la figlia di essere stato a trovare Torello, ancora ricoverato all'ospedale militare.

5. IT a Velia, cartolina ms. + busta, Firenze, 10 novembre 1926, 1 c. IT informa la figlia di essere in un momento di lavoro febbrile e che sarebbe dovuto andare a Pistoia, a Cerreto Guidi e a Vinci.

6. IT a Velia, lettera ms., Firenze, 26 maggio 1927, 1 c. IT si lamenta con Velia per la questione del compenso che gli sarebbe dovuto spettare per la vendita della villa fiorentina di Afrisio Vannacci, fratello di Amelia.

7. IT a Velia, lettera ms., Firenze, 14 giugno 1927, 2 cc. IT informa Velia di star migliorando di salute e continua a lamentarsi per l'affare della vendita della villa di suo cognato Afrisio. 
8. Velia a IT, cartolina ms., Viareggio, 27 luglio 1927, 1 c.

9. IT a Velia, lettera ms., Firenze, 27 novembre 1927, 1 c. IT informa Velia che il nuovo Segretario Generale era arrivato a presiedere l'Ufficio Provinciale dei Sindacati Fascisti e ne critica subito l'operato.

10. IT a Velia, lettera ms., Firenze, 21 luglio 1928, 2 cc. IT informa Velia di essersi informato circa i prezzi, a suo giudizio troppo cari, delle abitazioni sul litorale tirrenico e in particolare a Viareggio.

11. IT a Velia, lettera ms., Firenze, 26 luglio 1928, 2 cc. IT dice alla figlia di essere contento che lei e la famiglia sarebbero andati trovarlo in montagna a Casamarconi.

12. IT a Velia, lettera ms., Firenze, 30 luglio 1928, 2 cc. IT informa Velia dei suoi prossimi spostamenti in Val di Bisenzio e Val di Mugello.

13. IT a Velia, lettera ms., Firenze, 9 agosto 1928, 2 cc. IT dice a Velia di sentirsi tradito dal comportamento di Afrisio in merito alla questione della vendita della villa fiorentina.

14. IT a Velia, lettera ms., Firenze, 1 settembre 1928, 2 cc. IT dice a Velia di sentirsi meglio di salute, di non aver ancora riscosso le sue spettanze e si abbandona alla solita considerazione pessimista sulla vita.

15. IT a Velia, lettera ms., Firenze, 7 settembre 1928, 2 cc. IT dice a Velia che le avrebbe mandato un po' di denaro, di aver trovato molto lavoro da fare al suo ritorno in Federazione e che la sua posizione presso gli agricoltori fiorentini si stava facendo sempre più difficile.

16. IT a Velia, lettera ms., Firenze, 6 aprile 1929, 2 cc. IT informa Velia di essere stato a colloquio con il Prefetto di Pistoia per parlare del monumento ai Caduti di Lamporecchio. Parla anche della vendita della villa di Afrisio.

17. IT a Velia, lettera ms., Firenze, maggio 1929, 2 cc. IT dice a Velia di non sapersi più raccapezzare in merito alla questione della villa.

18. IT a Velia, lettera ms., Firenze, 10 maggio 1929, 2 cc. IT dice a Velia di essere a letto con la febbre ormai da dieci giorni e parla ancora in merito alla faccenda della villa.

19. Avv. Cesare Torricelli a IT, lettera ms., Firenze, 16 maggio 1929, 1 c. L'autore parla con IT di questioni testamentarie.

20. IT a Velia, lettera ms., Firenze, 22 maggio 1929, 2 cc. IT parla con la figlia ancora in merito alla questione della provvigione che gli sarebbe dovuta spettare sulla vendita della villa di Afrisio.

21. IT a Velia, lettera ms., Firenze, 26 maggio 1929, 2 cc. IT parla a Velia ancora in merito alla questione della villa.

22. IT ad Amelia, lettera ms., Firenze, 2 giugno 1929, 2 cc. IT dice alla moglie di star agendo per vie legali in merito alla questione della villa di Afrisio, di essere ancora malato, che sta spendendo molti soldi per curarsi e che lo stipendio gli è stato momentaneamente sospeso. 
23. IT ad Amelia, lettera ms., Firenze, 6 giugno 1929, 2 cc. IT parla con la moglie ancora in merito alla questione della villa.

24. IT a Velia, lettera ms., Firenze, 13 giugno 1929, 2 cc. IT parla a Velia ancora in merito alla questione della villa e si dice pronto ad avviare una causa legale.

25. IT a Velia, lettera ms., Firenze, 8 luglio 1929, 2 cc. IT parla a Velia delle sue condizioni di salute in miglioramento, della sua volontà di andare a Roma a risolvere la questione dello stipendio e ancora in merito alla questione della villa.

26. IT a Velia, lettera ms., Firenze, 29 luglio 1929, 2 cc. IT parla a Velia ancora in merito alla questione della villa, dicendo che avrebbe voluto parlare a tu per tu con Afrisio per chiarire la questione.

27. IT a Velia, lettera ms., Firenze, 11 agosto 1929, 2 cc. IT rimprovera Velia per non averlo sostenuto in merito alla questione della villa e di esser stata invece troppo comprensiva nei confronti dello zio Afrisio.

28. Busta contente due lettere:

1. IT a Velia, lettera ms., Firenze, 14 agosto 1929, 1 c. IT invita la figlia Velia a recarsi da lui, con tutte le lettere che le aveva inviato, per riparare ai litigi epistolari, far chiarezza sulla situazione e parlare in segreto.

2. IT ad Amelia, lettera ms., Firenze, 14 agosto 1929, 1 c. IT dice alla moglie di voler vendere un terreno agricolo di sua proprietà.

29. IT a Velia, lettera ms., Firenze, 18 agosto 1929, 1 c. IT dice a Velia di aspettare sue risposte in merito a un'eventuale decisione di Afrisio di recarsi a Firenze da lui per evitare una causa legale.

30. IT a Velia, lettera ms., Firenze, 2 settembre 1925, 1 c. IT invita la figlia Velia a ragguagliarlo su quanto le sta succedendo.

31. IT a Velia, lettera ms., Firenze, 23 settembre 1929, 1 c. IT dice a Velia di recarsi da lui a Firenze in compagnia di Afrisio per risolvere la questione delle provvigioni.

32. IT a Velia, cartolina ms., Trieste, marzo 1913, 1 c. Sul recto sono riprodotti in fotografia i dipinti presenti sul soffitto della sala di lettura del Circolo di Studi Sociali, Amore e Fecondità. Sul verso, IT parla a Velia in forma di poesia.

33. IT a Torello Chiappini, lettera ms., Lamporecchio, 2 luglio 1916, 2 cc. IT dice a suo genero Torello, impegnato in guerra, che con Afrisio stanno usando tutti i mezzi possibili per andare a trovarlo.

34. Tobia [?] a IT, cartolina ms., Campi Bisenzio, 26 dicembre 1917, 1 c. Il mittente invita IT a restituirgli una pezza che gli aveva precedentemente inviato e che IT aveva promesso di restituirgli.

35. Larini Pietro a IT, cartolina ms., Campo di Prigionia di Nerustadt Elenfurth - Vienna, 5 agosto 1917, 2 cc. 
36. IT a Torello Chiappini, cartolina ms., Firenze, 6 gennaio 1917, 1 c. IT informa Torello di essere stato alla posta e di non aver ricevuto ancora nulla.

37. IT a Velia, lettera ms. + busta, Firenze, 16 giugno 1925, 2 cc. IT invita la figlia a recarsi da lui in ufficio la mattina del 27 e che a Casamarconi sono già state messe due stanze a loro disposizione.

38. IT a Velia, lettera ms., s.l., s.d., 2 cc. IT cerca di confortare Velia, in quel momento ricoverata all'ospedale.

39. IT a Velia, lettera ms. + busta, Firenze, 9 settembre 1925, 2 cc. IT annuncia a Velia un suo prossimo rientro a casa a causa di impegni che avrebbe avuto a Cerreto Guidi e Lazzeretto.

40. IT a Velia, lettera ms. + busta, Firenze, 27 maggio 1925, 2 cc. IT informa Velia di essersi sottoposto alla radioscopia dello stomaco e che gli è stata diagnosticata un'ulcera gastrica. Il Dott. Daddi, a tal proposto, gli consigliava di sottoporsi a operazione finché era in tempo o lo inviava alle cure del Dott. Burci, Magnifico Rettore dell'Università di Firenze.

41. IT a Velia, lettera ms., Firenze, 1 maggio 1926, 2 cc. IT informa Velia di essere stato visitato dal Prof. Daddi il quale gli ha riscontrato un'ulcera benigna allo stomaco. Invita la figlia a non preoccuparsi e la informa di stare per cominciare un trattamento.

42. Busta contenente le seguenti lettere:

1. IT a Velia, lettera ms. + busta, Firenze, 8 maggio 1926, 2 cc. IT informa la figlia Velia che la salute va sempre migliorando e che l'emorragia è arrestata, anche se è sempre allettato e sottoposto a una rigida dieta.

2. Prof. Daddi al prof. Burgassi, lettera ms., Firenze, 1 maggio 1926, 1 c. Il Prof. Daddi informa il collega Burgassi circa lo stato di salute di IT e la terapia con cui lo sta trattando per la recente emorragia gastrica.

43. IT a Velia, lettera ms. + busta, Firenze, 9 maggio 1926, 2 cc. IT informa Velia di essere ormai degente da nove giorni, di essere fermo a letto per via dello stomaco e di essere in regime di digiuno forzato.

44. IT a Velia, lettera ms. + busta, Firenze, 11 maggio 1926, 2 cc. IT informa Velia circa le sue condizioni di salute e di star sbrigando tutta la corrispondenza d'ufficio da casa come se fosse in piena attività lavorativa. Sulle stesse carte è acclusa la risposta della figlia.

45. IT a Velia, lettera ms. + busta, Firenze, 13 maggio 1926, 2 cc. IT informa Velia di star bene e di non essere più in regime di digiuno.

46. IT a Velia, lettera ms. + busta, Firenze, 15 maggio 1926, 2 cc. IT informa Velia di essere allettato da cinque giorni per far risarcire l'ulcera allo stomaco, ma dice di sentirsi bene, tant'è che stava normalmente disbrigando la corrispondenza d'ufficio. 
47. IT a Velia, lettera ms. + busta, Firenze, 16 maggio 1926, 2 cc. IT informa Velia che ora gli si concede di mangiare e che, una volta tornato a casa, si sarebbe soltanto occupato della sua salute.

48. IT a Velia, lettera ms. + busta, Firenze, 20 maggio 1926, 2 cc. IT informa Velia di essere tornato in ufficio dopo ventiquattro giorni, ma solo per poche ore viste le ancor precarie condizioni di salute.

49. IT a Velia, lettera ms. + busta, Firenze, 31 maggio 1926, 1 c. IT informa Velia di essere in osservazione presso il Prof. Burci, il quale, prima di prendere decisioni in senso operatorio, vuol procedere con la radiografia dello stomaco.

50. Busta contenente i seguenti elementi:

1. IT a Velia, cartolina ms., Firenze, 1 giugno 1926, 1 c. IT informa Velia che quella mattina stessa aveva ricevuto la visita del Prof. Burci il quale gli aveva annunciato che avrebbe svolto l'operazione su di lui nell'arco di due-tre giorni.

2. Amelia a Velia, lettera ms., s.l. [Firenze], s.d. [1 giugno 1926], 2 cc. Amelia dice a Velia che poteva lasciare le bambine a una certa Lisa e le chiede, inoltre, di prenderle dei vestiti situati nella sua stanza.

51. IT a Velia, cartolina ms., Firenze, 19 giugno 1926, 1 c. IT dice a Velia di star gradualmente migliorando e riacquistando l'appetito.

52. IT a Velia, lettera ms. + busta, Firenze, 23 giugno 1926, 2 cc. IT informa Velia di star lentamente migliorando di salute pur prevedendo che la convalescenza potrà essere molto lunga. Dice di rispettare le prescrizioni mediche in quanto a riposo e nutrizione e che lo stomaco non gli sta provocando alcun dolore.

53. IT a Velia, lettera ms., Firenze, 27 giugno 1926, 2 cc. IT dice a Velia di star meglio e di aver fatto bene a rimanere a Firenze nel periodo post operatorio, perché continuamente seguito dai medici.

54. IT a Velia, lettera ms., Firenze, 5 luglio 1926, 2 cc. IT rassicura Velia circa le sue condizioni di salute, dice che sta lavorando molto e di essere in procinto di partire per Casamarconi.

55. IT a Velia, lettera ms., Firenze, 13 luglio 1926, 1 c. IT informa la figlia Velia di averle inviato le medicine per Barbara e di essere stato dal dentista, il quale avrebbe dovuto fargli alcuni interventi.

56. IT a Velia, lettera ms., Firenze, 21 agosto 1926, 2 cc. IT dice a Velia di essere tornato da un giro di propaganda in Valdarno, di averle inviato il busto riparato e le medicine per Barbara, e che non avrebbe fatto in tempo a recarsi da lei e gli altri a Casamarconi.

57. IT a Velia, lettera ms. + busta, Firenze, 12 dicembre 1926, 1 c. IT dice alla figlia Velia che nei giorni successivi sarebbe dovuto andare a Vitolini e che, con l'occasione, avrebbe fatto ritorno a casa.

58. IT a Velia, cartolina ms., Firenze, 30 gennaio 1927, 1 c. IT rimprovera la figlia di non avergli scritto nemmeno una riga. 
59. IT a Velia, lettera ms., Firenze, 14 febbraio 1927,1 c. IT dice alla figlia Velia che si sta riprendendo dall'attacco influenzale che lo aveva colpito.

60. IT a Velia, lettera ms., Firenze, 3 marzo 1927, 1 c. IT dice a Velia di aver visto con piacere la sua lettera sul tavolo della sua scrivania, perché il suo silenzio lo stava addolorando. Sul lavoro fornisce buone notizie. Il proposito di tornare a casa nella settimana successiva è stato rimandato a causa del suo impegno in prima persona nella stipula di due Patti di Lavoro (Boscaioli e Braccianti) a Firenze.

61. IT a Velia, lettera ms., Firenze, 22 marzo 1927, 1 c. IT dice a Velia di essere dispiaciuto del fatto che lei si faccia poco viva. Di salute dice di star molto meglio.

62. IT a Velia, lettera ms. + busta, Firenze, 1 aprile 1927, 1 c. IT informa Velia che Idalberta stava lentamente migliorando e che si poteva scongiurare ogni pericolo.

63. IT a Velia, lettera ms. + busta, Firenze, 15 aprile 1927, 2 cc. IT dice alla figlia Velia di aver preso l'influenza e che perciò avrebbe rimandato il ritorno a Lamporecchio.

64. IT a Velia, lettera ms. + busta, Firenze, 17 aprile 1927, 2 cc. IT dice alla figlia Velia di aver trascorso anche la Pasqua con la febbre.

65. IT a Velia, lettera ms. + busta, Firenze, 19 aprile 1927, 2 cc. IT dice a Velia di essere allettato da sei giorni e di aver bisogno di assoluto riposo.

66. IT a Velia, lettera ms. + busta, Firenze, 20 aprile 1927, 2 cc. IT dice a Velia di essere in via di miglioramento, anche se avrebbe dovuto fare degli accertamenti.

67. IT a Velia, lettera ms. + busta, Firenze, 23 aprile 1927, 2 cc. IT dice a Velia di star bene, la ringrazia per i buoni consigli e si abbandona ad alcune considerazioni sulla vita.

68. IT a Velia, lettera ms. + busta, Firenze, 27 aprile 1927, 1 c. IT invita la figlia a non preoccuparsi per la sua salute e le racconta l'intera evoluzione e il decorso della febbre che lo aveva colto di recente. Spera solo di poter presto tornare a lavoro.

69. IT a Velia, lettera ms., Firenze, 27 maggio 1927, 1 c. IT informa la figlia circa i suoi progressivi miglioramenti di salute.

70. IT a Velia, lettera datt. e ms., Firenze, 12 giugno 1927, 1 c. IT rassicura la figlia sulle sue condizioni di salute e la informa che avrebbe posticipato il rientro a $S$. Baronto.

71. IT a Velia, lettera ms., Roma, 21 giugno 1927, 1 c. IT informa la figlia di essere a Roma e in attesa di parlare con S. E. Martelli.

72. IT a Velia, cartolina ms., Firenze, 22 giugno 1927, 1 c. IT informa la figlia di essere tornato da Roma e di essere soddisfatto dell'incontro. 
73. IT a Velia, lettera ms., Firenze, 26 giugno 1927, 1 c. IT chiede alla figlia cosa abbia deciso per Viareggio e se sua madre abbia fissato la camera a S. Baronto.

74. IT ad Amelia, lettera ms., Firenze, 28 luglio 1927, 1 c. IT dice alla moglie Amelia di non sapere con precisione la data del suo ritorno a Lamporecchio perché impegnato nella risoluzione di due vertenze sindacali.

75. IT a Velia, lettera datt. e ms., Firenze, 23 settembre 1927, 1 c. IT chiede alla figlia di dargli sue notizie, visto che non aveva dato alcuna risposta alla sua precedente cartolina datata 10 settembre.

76. IT a Velia, lettera ms., Firenze, 19 ottobre 1927, 2 cc. IT dice alla figlia di star bene e che sta aspettando il momento di tornare a casa per tre o quattro giorni.

77. IT a Velia, lettera ms., Firenze, 30 ottobre 1927, 2 cc. IT dice alla figlia che spera di passare a casa per la festa dei Morti, ma la situazione a lavoro è molto caotica.

78. IT a Velia, lettera ms., Firenze, 2 novembre 1927, 1 c. IT racconta alla figlia le nuove condizioni di lavoro in ufficio.

79. IT a Velia, lettera ms., Firenze, 18 dicembre 1927, 2 cc. IT dice alla figlia di avere tante cose da raccontarle, fra le tante, riferisce di non essere più il Segretario dei Sindacati Colonici e Braccianti, ma avrebbe organizzato gli operai di tutte le classi.

80. IT a Velia, lettera ms. + busta, Firenze, 6 gennaio 1928, 2 cc. IT porge gli auguri alla figlia e famiglia, dopodiché si abbandona a considerazioni sulla vita e sulla fede.

81. IT a Velia, lettera ms., Firenze, 2 febbraio 1928, 2 cc. IT dice alla figlia di star meglio, di star ingrassando e che nei giorni successivi si sarebbe dovuto recare a Cerreto Guidi per un Congresso con i Segretari dei sindacati.

82. Velia a IT, lettera ms., s.l. [Lamporecchio], 29 marzo 1928, 1 c. Velia informa il padre di aver lasciato nel suo ufficio un bigliettino scritto da una signora di Casamarconi, che chiedeva un impiego per suo marito.

83. IT a Velia, cartolina ms., Roma, 5 maggio 1928, 1 c. Sul recto è presente la riproduzione di una foto in bianco e nero scattata all'esterno della Basilica di San Pietro e alla piazza antistante. Sul verso, IT informa la figlia che di lì a breve le avrebbe spedito dei soldi.

84. IT a Velia, lettera ms., Firenze, 2 giugno 1928, 2 cc. IT ringrazia la figlia per averlo assistito a Firenze durante la sua ultima febbre. Da Roma non era giunta ancora nessuna novità, come, d'altra parte, neanche in merito alla questione della compravendita della villa fiorentina di Afrisio.

85. IT a Velia, lettera ms., Firenze, 29 giugno 1928, 2 cc. IT informa la figlia Velia che intendeva prendere le ferie estive in agosto e che in quel periodo si sarebbe recato da lei a Viareggio. 
86. Velia a IT, lettera ms., Firenze, 4 luglio 1928, 2 cc. Velia informa il padre di aver deciso di trascorrere le vacanze estive a Viareggio e che gli avrebbe prenotato una camera qualora fosse stato intenzionato ad andare a trovarla.

87. IT a Velia, lettera ms. + busta, Firenze, 19 settembre 1928, 1 c. IT informa la figlia di aver chiesto di essere trasferito dalla Segreteria del Sindacato per l'Agricoltura e che l'avrebbero probabilmente destinato all'Ispettorato dell'Ufficio Provinciale di Collocamento dei Braccianti.

88. IT a Velia, lettera ms. + busta, Firenze, 19 marzo 1929, 1 c. IT dice alla figlia che nei giorni successivi si sarebbe recato a Pistoia per risolvere diverse questioni.

89. IT a Velia, lettera ms. + busta, Firenze, 6 maggio 1929, 1 c., IT informa la figlia di aver contratto l'influenza e di dover star allettato almeno cinque giorni. Riferisce di essersi incontrato con Afrisio a Firenze ma di non aver ottenuto nessuna novità sulla questione della villa.

90. IT a Velia, lettera ms. + busta, Firenze, 10 maggio 1929, 2 cc. IT informa la figlia di essere ancora allettato con la febbre e di essere pronto ad avviare una causa legale per la questione della villa di Afrisio.

91. IT a Velia, lettera ms. + busta, Firenze, 20 giugno 1929, 2 cc. IT dice alla figlia di aver ricevuto le 900 lire che gli ha spedito e che gli sarebbero bastate per tutto il mese. In merito alla questione della quota sulla mediazione nella vendita della villa di Afrisio, chiede che quest'ultimo gli invii almeno altre 1000 lire.

92. IT a Velia, lettera ms., Firenze, 1 ottobre 1929, 1 c. IT informa la figlia circa le sue condizioni di salute, ovvero che si sente meglio ma ha ancora dei fastidi di cui descrive i sintomi. Inoltre annuncia che a breve sarebbe uscito il suo libro, Il Calendario Agricolo Fascista.

93. IT a Velia, lettera ms. + busta, Firenze, 8 ottobre 1929, 1 c. IT informa la figlia di essere ancora in attesa di risposte circa l'arbitrato proposto dal suo avvocato per regolare la questione della villa.

94. IT a Velia, lettera ms., Firenze, 16 dicembre 1929, 1 c. IT chiede alla figlia di passare al Mancini una copia del ricorso contro il Talini, presidente del Comitato pro monumento ai Caduti di Lamporecchio. Il Mancini poi, avrebbe dovuto sottoporre il ricorso ai fascisti componenti il Comitato, raccogliere le loro firme e sottoporre il tutto all'attenzione del Prefetto.

95. IT a Velia, lettera ms., s.l., s.d., 1 c. IT informa la figlia di essere impegnato con il rinnovo di tre Patti di Lavoro (Coloni, Braccianti e Boscaioli) a Gambassi e che sarebbe passato da Lamporecchio solo per un saluto. 
96. IT a Velia, lettera ms., s.l., s.d., 2 cc. IT informa la figlia di non riuscire a tornare a casa come aveva preventivato.

97. IT a Velia, lettera ms., Firenze, 11 febbraio 1925, 1 c. IT dice alla figlia che essendo molto impegnato con il lavoro non sa quando sarebbe potuto tornare a casa.

98. IT ad Amelia, lettera ms., s.l., s.d., 1 c. IT informa Amelia di essere impegnatissimo nell'ufficio del Sindacato degli Agricoltori per concordare il Patto di Lavoro dei Braccianti e che non sa quando potrà rientrare a Lamporecchio.

2. Scatola con scritta «Astoria» contenente documenti di corrispondenza varia:

1. IT ad Amelia, lettera ms., Prato, 27 febbraio 1905, 2 cc. IT informa Amelia che sta tornando da Alesella, che è diretto a Firenze, che ha maturato scarsi guadagni con il lavoro e le chiede di inviargli la posta a Firenze.

2. Fortunato Chiti ad Amelia, lettera ms., Poggio a Caiano, 24 maggio 1930, 1 c. L'autore avverte la destinataria di aver informato Sofia circa le precarie condizioni di salute di IT e di essere stato con quest'ultima alla Federazione per ottenere, senza successo, i soldi spettanti a IT. Chiti quindi chiede ad Amelia, per conto di IT, un anticipo di $£$ 500.

3. Per i fausti sposi della Signorina Velia Targioni col Signor Torello Chiappini, Alcuni amici offrono, carte mss., s.l. [Lamporecchio], s.d. [20 novembre 1913], 2 cc. Bozza di componimento poetico dedicato allo sposalizio tra Velia Targioni e Torello Chiappini (cfr. anche 5.66.24).

4. IT a Velia, cartolina ms., Pola-Trieste, 19 marzo 1913, 1 c. Sul recto, immagine di due amanti al telefono. Sul verso, messaggio scritto in versi (quattro strofe da quattro quartine).

5. IT a Velia, cartolina ms., Trieste, 20 marzo 1913, 1 c. Sul recto, immagine di gatti. Sul verso, messaggio scritto in versi (due quartine) dedicato alla passione di Velia per i felini.

6. IT a Velia, cartolina ms., Pavana, 28 agosto 1910,1 c. Sul recto, fotografia che ritrae una via e parte della popolazione delle Montagne Pistoiesi - Pavana. Sul verso, messaggio in versi (cinque quartine).

7. IT a Velia, cartolina ms., Herisau (Svizzera), 17 agosto 1912, 1 c. Sul recto, fotografia che ritrae Villa Breitenmoser a Herisau, in Svizzera. Sul verso, l'autore informa la destinataria che quella ritratta sulla cartolina è il luogo in cui quella sera stessa avrebbe tenuto un'accademia. Le sue condizioni di salute sono migliorate e ha inviato due vaglia.

8. IT a Velia, cartolina ms., Roma, 17 dicembre 1930, 1 c. Sul recto, fotografia che ritrae dei barcaioli fermi su una sponda del fiume Te- 
vere e sullo sfondo Castel Sant'Angelo e San Pietro. Sul verso, IT, con cinque sestine, informa Velia che il giorno 28 sarebbe ritornato a casa.

9. IT a Velia, cartolina ms., Lago di Lugano - Gandria, 11 agosto 1912, 1 c. Sul recto, fotografia a colori che ritrae il paesino di Gandria verso la Val di Solda sul Lago di Lugano. Sul verso, IT informa Velia che il suo pensiero è corso a lei e alla madre.

10. IT a Velia, cartolina ms., Venezia, 3 gennaio 1912, 1 c. Sul recto viene ritratta la chiesa e una parte della piazza di S. Marco. Sul verso, due quartine e due terzine con cui IT descrive a Velia la bellezza della città.

11. [IT] a Velia, cartolina ms., Firenze, 27 dicembre 1922, 1 c. Sul recto, fotografia che ritrae la Loggia dell'Orcagna in Piazza della Signoria a Firenze. Sul verso, [Idalberto?] ringrazia Velia per il pensiero gentile e da lui molto gradito.

12. IT a Velia, cartolina ms., Bologna, 11 novembre 1922, 1 c. Sul recto sono ritratte le due torri di Bologna. Sul verso, messaggio di Idalberto alla figlia Velia in cui l'autore informa la destinataria di essere appena uscito dal Convegno Nazionale delle Corporazioni e definisce Bologna, dopo Roma, «la più austera città d'Italia».

13. IT a Velia, cartolina ms., Massa Marittima, 9 febbraio 1914, 1 c. Sul recto, fotografia del monumento a Garibaldi di Massa Marittima e di alcuni paesani radunati lì attorno. Sul verso, messaggio di Idalberto alla figlia Velia in cui l'autore raccomanda alla destinataria massimo riguardo per se stessa visto il suo stato interessante.

14. IT a Velia, cartolina ms., Trieste, [3] gennaio 1912, 1 c. Sul recto, fotografia della stazione Meridionale di Trieste. Sul verso, IT informa la figlia circa la bellezza della città e che presto avrebbe avuto notizie sull'esito delle sue accademie dai giornali di Trieste e Pistoia.

15. IT a Velia, cartolina ms., Vallombrosa, 5 settembre 1911, 1 c. Sul recto, fotografia panoramica del Saltino in Vallombrosa. Sul verso, IT scrive versi con cui informa Velia circa le cure che sta facendo nella zona di Saltino a Vallombrosa.

16. IT a Velia, cartolina ms., Massa, s.d., 2 cc. Sul recto, fotografia raffigurante una delle fasi del trasporto dei marmi a Massa. Sul verso, IT saluta la figlia Velia e la moglie.

17. IT ad Amelia, cartolina ms., Zurigo, 19 agosto 1912, 1 c. Sul recto, fotografia a colori raffigurante la città di Zurigo. Sul verso, IT informa Amelia circa il suo buono stato di salute e di averle spedito un vaglia per sistemare alcune questioni.

18. IT a Velia, cartolina ms., Kursaal de Montreux (Glion), 6 agosto 1913,1 c. Sul recto, fotografia a colori raffigurante uno scorcio della città svizzera di Glion. Sul verso, IT informa Velia di aver ricevuto la 
sua cartolina e si rammarica del fatto che le sue precedenti tre lettere inviate dalla Svizzera non le siano ancora state recapitate.

19. IT ad Amelia, cartolina ms., s.l., 27 dicembre 1921, 1 c. Sul recto, disegno raffigurante contadini in cammino sulla strada che porta a una chiesa posta su un colle. Sul verso, componimento poetico dedicato da IT ad Amelia.

20. IT a Velia, lettera ms., Firenze, 27 agosto 1923, 1 c. IT dice a Velia di essere contento che lei e gli altri familiari stiano tutti bene. Ma si premura del fatto che la figlia gli dica sempre la verità a tal proposito.

21. IT a Velia, minuta ms., Zurigo, 23 agosto 1912, 4 cc. IT invia a Velia una poesia di 22 quartine sul treno Losanna-Zurigo e dedicata a lei.

22. Scritti per la defunta figlia Rita, carte mss., s.l., s.d., 3 cc. Scritti incompleti (manca la prima parte) dedicati a Rita, figlia prematuramente scomparsa.

23. Foto di IT con la figlia Idalberta, busta + ritaglio di fotografia, Firenze, s.d. [1929-1930], 1 c. Ritaglio di fotografia in bianco e nero raffigurante IT vestito elegantemente con un abito nero e cappello, seduto su uno sgabello, con un cane adagiato ai suoi piedi e affiancato alla sua sinistra dalla figlia Idalbertina, vestita con un abito bianco.

24. Alla Nobile e benemerita Signora Laura T. Merrick pel suo fausto ritorno alla Villa di Papiano, le bambine Velia e Rita Targioni in segno d'affetto e devozione perenni offrono, pergamena stampata, Empoli, Tip. Traversari, giugno 1905, 2 cc.

25. Per le fauste nozze dell'ottimo giovine operaio Torello Chiappini colla fanciulla Velia Targioni, pergamena a stampa, s.l. [Lamporecchio], 20 novembre 1913, 2 cc.

26. Busta da lettera verde con la seguente iscrizione: «Signora Velia Chiappini Targioni/ Lamporecchio».

1. Velia a Torello, lettera ms., s.l. [Viareggio], 9 luglio 1922, 2 cc. Velia chiede al marito di spedirle dei denari per permetterle di mantenere sé e i ragazzi nel luogo di villeggiatura marittima in cui si trova.

2. Velia a Torello, lettera ms., s.l. [Viareggio], 16 luglio 1924, 1 c. Velia rimprovera il marito per le espressioni da lui usate nella lettera che le aveva inviato e lo invita ancora una volta a inviare denari per permettere ai figli di continuare la vacanza terapeutica in località balneare.

3. IT a Velia, lettera ms., Firenze, 22 giugno 1923, 1 c. IT informa la figlia circa il duro lavoro che sta svolgendo e che presto l'avrebbe raggiunta a Viareggio. 
4. IT a Velia, lettera ms., Firenze, 26 giugno 1923, 1 c. IT informa Velia di quanto i litigi tra lei e il marito abbiano ripercussioni sul suo stato d'animo e di salute.

5. IT ad Afrisio, minuta ms., Firenze, 26 giugno 1923, 2 cc. IT informa Afrisio circa i continui litigi tra Velia e Torello, situazione che procura a IT grande rabbia e strazio e allega le lettere che Velia gli ha spedito a riguardo.

6. IT a Velia, lettera ms., Firenze, 15 settembre 1923, 1 c. IT informa la figlia circa il suo stato di salute. Le chiede di tenerlo aggiornato sulla sua situazione senza nascondergli nulla.

7. IT a Velia, lettera ms., Casamarconi, 8 agosto 1928, 2 cc. IT informa la figlia di essere giunto abbastanza in fretta a Casamarconi e di avere a disposizione una stanza in più che potrebbe ospitarla. Racconta, inoltre, di aver passato una serata in paese e di aver salutato dei vecchi amici.

8. IT a Velia, lettera ms., Pontassieve, 23 maggio 1929, 2 cc. IT informa Velia di essere oberato di lavoro e di soffrire sempre più di dolori allo stomaco.

27. Busta celestina della Federazione Provinciale dei Sindacati Fascisti Sezione di Firenze. Contiene i seguenti elementi:

1. IT a Velia, lettera ms., Firenze, 13 luglio 1923, 1 c. IT informa la figlia circa il suo stato di salute e le riferisce che l'indomani non sarebbe tornato a Lamporecchio perché chiamato a risolvere importanti vertenze sindacali.

2. IT a Velia, lettera ms., Firenze, 5 febbraio 1924,1 c. IT informa la figlia Velia di non esser riuscito a concludere alcun affare. Alterna poi giudizi ottimistici e pessimistici sulla vita.

3. IT a Velia, lettera ms., Pistoia, 18 luglio 1924, 2 cc. IT informa Velia di essere partito da Lamporecchio per concludere alcuni piccoli affari in varie località. Per gli affari più importanti, invece, le cose continuavano a non andar bene.

4. IT a Velia, lettera ms., Firenze, 26 dicembre 1924, 1 c. IT informa la figlia Velia circa la sua prossima venuta per trascorrere le feste in famiglia. Il 2 gennaio sarebbe dovuto ripartire a causa lavoro.

5. IT a Velia, lettera ms., Firenze, 10 gennaio 1925, 1 c. IT informa la figlia Velia che verso la fine del mese si sarebbe recato a Lamporecchio e vi si sarebbe intrattenuto per diversi giorni per questioni sindacali. Dà istruzioni alla figlia per la consegna di alcune sue lettere e si congeda da lei consigliandole di stare di buon animo e di consolarsi con i bambini.

6. IT a Velia, lettera ms. + ritaglio di giornale, Firenze, 24 gennaio 1925, 2 cc. IT informa Velia dei risultati positivi che sta otte- 
nendo nel Sindacato Fascista per l'Agricoltura. Scrive inoltre che alla fine del mese sarebbe tornato a Lamporecchio sempre per risolvere questioni sindacali in diversi paesi delle vicinanze. Un ritaglio di giornale è stato infilzato con la lettera, probabilmente a posteriori e da terzi. L'articolo riguarda La querela Targioni "Bandiera del Popolo", datato 29 gennaio 1925.

7. IT a Velia, lettera ms., Firenze, 5 giugno 1925, 2 cc. IT informa Velia che sarebbe di lì a poco andato in montagna, probabilmente a Casamarconi, dove aveva trascorso parte dell' infanzia.

8. IT a Velia, lettera ms., Firenze, 1 agosto 1925, 1 c.

IT informa la figlia di essere passato dall'ospedale e di non aver potuto ricevere informazioni in merito al suo busto. Circa la sua salute dice di star meglio.

9. IT a Velia, lettera ms., Firenze, 3 agosto 1925, 1 c.

IT informa Velia che il Dott. Trinci le consigliava di tenere il busto fino al $35^{\circ}$ giorno. Circa il suo stato di salute dice di essere in costante miglioramento e di avere ottenuto il permesso per tornare in montagna.

28. IT a Velia, lettera ms., Firenze, 8 luglio 1923, $3 \mathrm{cc}$.

IT informa Velia di aver avuto male a un occhio e che nonostante avesse adoprato alcuni rimedi il dolore non gli era ancora passato. Oltre a ciò racconta che stava per essere truffato nella riscossione di due buoni del tesoro.

29. IT a Velia, cartolina ms., Firenze, 27 luglio 1923, 1 c. IT informa Velia che, impegni permettendo, sarebbe andato a trovarla al mare.

30. IT a Velia, cartolina ms., Firenze, 22 settembre 1923, 1 c. IT parla delle sue condizioni di salute in miglioramento e dei suoi prossimi impegni di lavoro col Sindacato. Si lamenta, inoltre, di non ricevere nuove dalla figlia. Lettera incompleta.

31. IT a Velia, cartolina ms. + busta, Firenze, 30 aprile 1924, 1 c. IT informa Velia che sta aspettando notizie da Empoli prima di andare a Porciano a definire la questione dell'illuminazione pubblica.

32. IT a Velia, lettera ms., Firenze, 3 maggio 1924, 1 c. IT informa Velia che il Consiglio di disciplina e il Direttorio della Federazione Fascista avevano esaminato il ricorso da lui presentato in merito alla sua espulsione dai Sindacati Fascisti e di essere stato riammesso.

33. IT a Velia, lettera ms., Firenze, 7 dicembre 1925, 1 c. IT informa Velia circa il suo stato di salute dicendole che il freddo di quei giorni lo faceva star peggio.

34. IT a Velia, lettera ms., Firenze, 24 dicembre 1925, 1 c. IT allega dei campioni di biscotti, caramelle e gianduiotti che avrebbe portato personalmente a casa di Velia e dei nipoti Rita, Lida e Idalbertino nei giorni successivi al Natale. 
35. IT a Velia, lettera ms., Firenze, 26 aprile 1926, 2 cc. IT informa Velia di non star molto bene di salute. Delega inoltre la figlia di recapitare delle lettere.

36. IT a Velia, lettera ms., Firenze, 16 aprile 1927, 1 c. IT informa la figlia circa il suo stato di salute poco buono.

37. IT a Velia, lettera ms., Firenze, 28 marzo 1928, 2 cc. IT dice alla figlia di cominciare a sentire nostalgia di casa e la informa che sarebbe tornato nei giorni precedenti la Pasqua.

38. IT a Velia, lettera ms., Firenze, 18 luglio 1928, 2 cc. IT informa la figlia che sarebbe andato a trovarla a Viareggio. La invita inoltre a prestare attenzione al tifo che si stava diffondendo in quella città.

39. IT a Velia, lettera ms., Firenze, 25 agosto 1928, 1 c. IT informa la figlia circa le sue numerose attività. Sul fronte politico, l'impegno più gravoso è quello della preparazione delle liste elettorali per le nuove elezioni.

40. IT a Velia, lettera ms., Firenze, 11 settembre 1928, 1 c. IT dice alla figlia di star bene di salute, parla del lavoro e della questione del Monumento ai Caduti.

41. IT a Velia, lettera ms., Firenze, 2 ottobre 1928, 1 c. IT dice alla figlia di aver trascorso giorni di grande tensione.

42. IT a Velia, lettera ms. + busta, Firenze, 18 dicembre 1928, 2 cc. IT informa Velia circa le sue condizioni di salute dicendo che doveva riguardarsi ancora qualche giorno e che il Dott. Daddi lo aveva trovato migliorato. Si evince inoltre che c'è un contenzioso fra lui e la Federazione provinciale dei Sindacati Fascisti.

43. IT a Velia, lettera ms., Firenze, 22 settembre 1929, 1 c. IT informa Velia circa i suoi affari con Afrisio e che stava attendendo l'arrivo di una lettera dell'On. Razza.

44. IT a Velia, cartolina ms., Roma, 8 novembre 1929, 1 c. Sul recto è presente una foto frontale, in bianco e nero, scattata all'Altare della Patria a Roma. Sul verso, IT informa Velia di essere stato riconfermato in servizio, con il solito stipendio, anche se al momento non gli era possibile lavorare.

45. IT a Velia, cartolina ms., s.l. [Firenze], s.d., 1 c. Sul recto è presente un disegno raffigurante una donna con le fattezze e gli abiti di una donna di campagna, con un cesto di vimini contenente uova colorate e con la scritta «Buona Pasqua». Sul verso, IT augura buona Pasqua ai nipotini e informa Velia di essere allettato con la febbre alta.

46. Foto ritagliata raffigurante IT, fotografia in $b / n$, s.l., s.d., $1 \mathrm{c}$.

47. Foto-cartolina con barca a vela, fotografia in b/n, s.l., s.d., $1 \mathrm{c}$.

48. Foto-cartolina raffigurante l'esterno di uno stabilimento portuale, fotografia in b/n, s.l., s.d., $1 \mathrm{c}$. 
49. Foto-cartolina raffigurante un corteo patriottico nello spazio antistante il palazzo comunale di Lamporecchio, fotografia in $\mathrm{b} / \mathrm{n}, \mathrm{s.l}$., s.d. [1910-1920], 1 c. Tra i partecipanti al corteo sembra di riconoscere il volto di IT.

50. IT a Velia, busta e lettera mss., Firenze, 13 gennaio 1930, 2 cc. IT informa Velia circa le sue precarie condizioni di salute e di aver fatto una nuova richiesta di riscossione degli arretrati che il Sindacato gli doveva.

51. IT a Velia, busta e lettera mss., Firenze, 5 maggio 1930, 2 cc. IT dice alla figlia che nei giorni successivi avrebbe preso il treno per Pistoia per andare a trovare lei e Amelia.

52. Sofia a Velia, busta e lettera mss., Firenze, 27 febbraio 1930, 2 cc. Sofia informa Velia del fatto che IT sta tornando da Roma, dove è stato a chiedere alla Federazione che gli venissero corrisposti dei soldi che gli spettavano, e che la sua salute era sempre molto debole.

53. IT a Velia, lettera ms., Firenze, 24 ottobre 1929, 1 c. IT informa la figlia circa il suo pessimo stato di salute e di essere inquieto per dei mancati pagamenti.

54. IT a Velia, busta e lettera mss., Firenze, 7 aprile 1930, 2 cc. IT informa la figlia circa il suo precario stato di salute. Parla ancora dei mancati pagamenti e della questione della villa di Afrisio.

55. IT a Velia, lettera ms., Firenze, 26 aprile 1930, 2 cc. IT informa la figlia circa le sue ancora precarie condizioni di salute. Anche la situazione finanziaria non è delle migliori. Sta pensando di andare a Roma alla Federazione per chiedere i soldi che gli spettano.

56. IT a Velia, lettera ms., Firenze, 7 novembre 1923, 1 c.

IT informa la figlia di aver appreso quanto accade tra lei e suo marito e la invita a non tenerlo all'oscuro delle sue vicende relazionali.

57. Velia a IT, lettera ms., s.l. [Lamporecchio], 7 novembre 1923, 2 cc. Velia informa IT circa i litigi continui col marito. Allega al padre una lettera del marito (non presente) e lo invita a non reagire d'impulso.

58. IT a Velia, lettera ms., Firenze, 14 novembre 1923, 1 c. IT informa Velia di aver preparato una lettera destinata al marito di lei, Torello, in cui gli dà gli ultimi avvertimenti prima di procedere per le vie di fatto.

59. IT a Velia, lettera ms., Firenze, 14 marzo 1930, 1 c. IT informa Velia di esser tornato da Roma con sole promesse e senza denari e di aver passato una visita medica dal Prof. Daddi, il quale gli aveva prescritto delle nuove cure.

60. Sofia a Velia, lettera ms., 9 maggio 1930, 2 cc. Sofia informa Velia circa lo stato di salute di IT, che è stato molto malato e che è stato preso in cura dal prof. Burgassi e dal Dottor Daddi, i quali gli hanno prescritto una dieta. 
61. IT a Velia, lettera ms., Firenze, 1 aprile 1930, 1 c. IT informa Velia circa le sue precarie condizioni di salute ed economiche.

62. Bozza di testamento, carta datt., Firenze, 20 novembre 1929, 1 c. In questa versione, IT lascia in eredità a Sofia metà delle sue sostanze.

63. IT a Velia, lettera ms., Firenze, 2 giugno 1926, 2 cc. IT scrive alla figlia prima di un intervento chirurgico dicendo che quelle che stava scrivendo sarebbero potute essere le sue ultime parole.

64. IT a Velia, lettera ms., Firenze, 17 aprile 1930, 2 cc. IT informa Velia circa le sue precarie condizioni di salute.

65. IT a Velia, lettera ms., Firenze, 21 marzo 1929, 2 cc. IT informa Velia circa le sue precarie condizioni di salute.

66. Sofia a Velia, lettera ms., s.l. [Firenze], 2 ottobre 1930, 2 cc. Sofia informa Velia sulla questione dell'eredità monetaria del defunto padre, in particolare del libretto. A fine lettera è Idalberta a scrivere a Velia, ringraziandola per le 50 lire che le ha regalato.

67. Idalberta a Velia, lettera ms., s.l. [Firenze], s.d. [post 25 maggio 1930], 1 c. Idalberta dice a Velia di essere dispiaciuta del fatto che lei e famiglia non sarebbero andate a Firenze, dove avrebbero potuto parlare del defunto padre.

68. IT a Torello Chiappini, lettera ms., Firenze, 14 novembre 1923, 4 cc. IT critica aspramente Torello per la sua condotta e per non esser stato riconoscente per tutti gli sforzi fatti per lui e la sua famiglia.

69. IT a Velia, lettera ms., Casa [Lamporecchio o Firenze?], 16 settembre 1925, 1 c. IT informa Velia che da quel momento in avanti non avrebbe più sostenuto le sue spese extra. Alla fine della lettera acclude un elenco dettagliato di tutte le spese sostenute e che desidera siano rimborsate.

70. IT a Velia, lettera ms., Lamporecchio, s.d., 1 c. IT invita Velia a non nascondergli nulla.

71. Sofia a Velia, lettera ms., s.l. [Firenze], 20 maggio 1930, 1 c. Sofia informa Velia che il Dottor Burgassi ha scritto una lettera a IT in cui lo invita a essere più ottimista circa le sue condizioni di salute.

72. IT a Velia, lettera ms., Firenze, 11 novembre 1923, 1 c. IT riferisce alla figlia quanto lo abbia irritato l'ultima lettera inviatagli da Torello Chiappini.

73. IT a Torello, lettera ms., Lamporecchio, 15 maggio 1924, 2 cc. IT rivolge aspre critiche al genero per la sua condotta irrispettosa nei confronti suoi e di sua figlia Velia.

74. IT a Vannucci, minuta ms., s.l., s.d., 1 c. L'autore esorta il destinatario a citare dei signori in giudizio e a pagargli delle provvigioni.

75. IT a Velia, lettera ms., Firenze, 21 agosto 1921, 1 c. IT informa Velia circa il suo stato di salute in ripresa. 
76. IT a Velia, busta + lettera ms., Roma, 3 dicembre 1929, 2 cc. IT informa la figlia di essere da qualche giorno a Roma per sistemare la sua posizione economica (vantava un credito verso la Federazione dei Sindacati Fascisti per l'Agricoltura).

77. IT a Velia, busta + lettera datt., Firenze, 19 gennaio 1930, 2 cc. IT informa Velia sulle sue condizioni di salute e sul fatto che ancora sta attendendo dei soldi da Roma. Le dà inoltre varie istruzioni.

78. Testamento di morte di IT, carte dattiloscritte, Firenze, 26 giugno $1930,4 \mathrm{cc}$.

79. IT a Velia, lettera ms., Firenze, 30 marzo 1929, 2 cc. IT informa Velia circa le condizioni di salute della piccola Idalberta, vittima di febbri alte e polmonite. La situazione era evidentemente molto disperata al punto che IT prega e si rivolge a Dio come forse non aveva mai fatto prima.

80. Sofia a IT, busta + lettera ms., Firenze, 13 maggio 1930, 2 cc. Sofia si rivolge a IT dicendosi preoccupata, come sempre, per la sua salute. Lo invita a seguire le prescrizioni mediche e lo informa che gli avrebbe spedito i medicinali di cui avrebbe avuto bisogno.

81. F. Gasperini a Targioni, busta + lettera ms., Pistoia, 16 maggio 1930, $1 \mathrm{c}$. L'autore si dice addolorato per aver appreso della malattia di IT e insieme agli auguri di pronta guarigione invia dei medicinali.

82. Sofia a Velia, lettera ms., Firenze, 21 luglio 1929, 2 cc. Sofia avverte Velia che IT è intenzionato ad andare in città per risolvere la questione della villa di Afrisio. È molto preoccupata per le sue condizioni di salute e del fatto che IT sembra non curarsi delle prescrizioni mediche.

83. Sofia a IT, busta + lettera ms., Firenze, 17 maggio 1930, 2 cc. Sofia prega IT, in quel momento a Lamporecchio, di fargli avere al più presto notizie circa il suo stato di salute e a tal proposito gli consiglia di farsi visitare dal Dottor Lauda, medico di Vinci.

84. Sofia e Idalberta a IT, busta + lettera ms., Firenze, 15 maggio 1930, $3 \mathrm{cc}$. Sofia invita IT a darle sue notizie e a prendersi adeguatamente cura di sé.

85. Idalberta e Sofia a Velia, busta + lettera ms., Firenze, 2 giugno 1930, $2 \mathrm{cc}$. IT è morto da poco e Idalberta sollecita Velia a cercar di risolvere il contenzioso aperto tra il Sindacato Fascista per l'Agricoltura e IT, il quale reclamava dei mancati pagamenti. Sofia si limita a scrivere che nella valigia di IT c'era tutto l'incartamento che riguardava i suoi affari con i sindacati.

86. Idalberta e Sofia a Velia, lettera ms., Firenze, 7 luglio 1930, 3 cc. Il dolore per la perdita del padre è ancora forte e Idalberta scrive alla sorellastra che il giardino che curava sempre il padre è in fiore. Sofia, invece, informa Velia delle questioni burocratiche che riguardavano il defunto compagno, ovvero del mancato pagamento di alcune sue competenze al Sindacato per l'Agricoltura. 
87. IT a Velia, busta + lettera ms., Roma, 3 febbraio 1930, 2 cc. IT informa la figlia di essere a Roma da cinque giorni, in attesa di avere un colloquio con l'on. Razza presso la Confederazione dei Sindacati Fascisti per l'Agricoltura, per la solita questione dei mancati pagamenti.

88. IT a Velia, busta + lettera ms., Firenze, 31 marzo 1929, 1 c. IT annuncia a Velia che la Pasqua non potrà trascorrerla con loro a Lamporecchio a causa di una brutta febbre che ha colpito Idalbertina. Riferisce inoltre dell'avvenuto incontro in merito alla vicenda della villa di Afrisio a Firenze.

89. Sofia a Velia, lettera ms., Firenze, 27 dicembre 1928, 2 cc. Sofia informa Velia circa l'imminente venuta di IT a Lamporecchio, dando notizie sulle condizioni di salute del compagno e sulla dieta che è costretto a seguire.

90. Busta di lettera destinata a Velia, busta, Firenze, 24 giugno 1929, 1 c.

91. Busta dell'"Istituto Toscano per analisi mediche e ricerche di biologia e chimica applicata/ Firenze - Via Cavour 28”, busta + oggetti interni, Firenze, s.d., 1 c. All'interno si trovano due tipi di fiori secchi e una ciocca di capelli castani tenuti con uno spago.

92. Sofia e Idalberta a Velia, busta + lettere mss., Firenze, 28 giugno 1930,4 cc.

93. Idalberta a Velia, busta + lettera ms., Firenze, 26 maggio 1930, 2 cc.

94. Idalberta a Velia, busta + lettera ms., Firenze, 12 giugno 1930, 2 cc. Idalberta invia alla sorellastra due foto del defunto padre, per scegliere quella più adatta da apporre sulla tomba e la informa del fatto che la mamma, Sofia, sia andata per l'ennesima volta alla Federazione, non ottenendo però alcuna notizia sulla questione dei mancati pagamenti.

95. Sofia a Velia, busta + lettera ms., Firenze, 24 giugno 1929, 2 cc. Sofia informa Velia che i medici Burgassi e Daddi hanno riscontrato in IT una forma tubercolare e lo hanno perciò invitato a stare a riposo.

96. Idalberta a Velia, busta + lettera ms., Firenze, 30 maggio 1930, 2 cc. Idalberta ringrazia Velia per il sostegno dato a lei e alla madre. Confessa alla sorellastra che tornare nella casa dove il padre non farà più ritorno, perché deceduto, le fa male.

97. IT a Velia, lettera ms., Firenze, 22 giugno 1929, 2 cc. IT rassicura Velia circa le sue condizioni di salute. Si dice poi rammaricato per il fatto che fino a quel momento lei avesse nutrita una sorta di avversione per Idalberta.

98. Biglietto di auguri natalizio di Idalberto Chiappini ai genitori Velia e Torello, busta + cartolina ms., Lamporecchio, 25 dicembre 1927, 2 cc. Frontespizio decorato e con sagoma cartacea di un angioletto.

99. Lida a Sofia, lettera ms., s.l., 4 febbraio 1931, 2 cc. 
100. Sofia e Idalberta a Velia, lettera ms., Firenze, 27 febbraio 1931, 2 cc.

101. Sofia e Idalberta a Velia, lettera ms., Firenze, 12 marzo 1931, 2 cc.

102. Sofia e Idalberta a Velia, lettera ms., Firenze, 25 marzo 1931, 2 cc.

103. Sofia e Idalberta a Velia, lettera ms., Firenze, 30 marzo 1931, 3 cc.

104. Abramo Randazzi [?] a Velia e Amelia, lettera ms., Zalana, 30 marzo 1931,2 cc.

105. Sofia e Idalberta a Velia, lettera ms., Firenze, 24 maggio 1931, 3 cc.

106. Sofia a Velia e di Idalberta a Velia e Lida, lettera ms., Firenze, 8 giugno 1931, $4 \mathrm{cc}$.

107. Sofia a Velia Targioni e di Idalberta a Velia, Lida e Idalberto Chiappini, lettera ms., Firenze, 30 giugno 1931, 4 cc.

108. Copertina grigia contenente i seguenti elementi:

1. Foto-cartolina raffigurante Idalberta a braccio teso, Firenze, settembre 1931, $1 \mathrm{c}$.

2. Foto-cartolina raffigurante Idalberta con Buby in braccio, Firenze, settembre 1931, 1 c.

3. Foto-cartolina raffigurante Idalberta vicina a un pozzo, Firenze, settembre 1931, 1 c.

4. Tessera del PNF di Lamporecchio di IT, tessera a stampa e datt., Lamporecchio, 1929, 2 cc.

5. Foto-cartolina raffigurante IT, s.l., s.d., 1 c.

109. Foto di soldati della Prima guerra mondiale, fotografie, s.l., s.d., 2 cc.

3. «Lettere»-Cartellina grigia contenente lettere di cui IT è destinatario.

1. Capo dell'Ufficio Politico della $95^{\circ}$ Legione Marzocco, Firenze, 27 dicembre 1929, 2 cc. Il mittente invita IT a passare dal suo ufficio per comunicargli notizie positive circa una risposta data da S. E. Tieraffi.

2. «Lettere Familiari» - Cartellina rossa contenente le seguenti lettere:

1. Torello a Sofia, lettera ms., Lamporecchio, 8 ottobre 1929, 1 c. Si parla delle cose familiari, delle figlie Lida e Rita e di Idalberta, figlia di Sofia e IT.

2. Velia a IT, lettera ms., Lamporecchio, 8 ottobre 1929,1 c. Si parla del contenzioso tra IT e altri due individui in merito alla questione della vendita della villa di Afrisio.

3. Velia a IT, lettera ms., Lamporecchio, 29 ottobre 1929, 2 cc. Velia si rammarica per la salute cagionevole del padre a cui consiglia il nome di un medico per farsi visitare. Si parla, inoltre, ancora in merito alla questione dei documenti che avrebbe dovuto trovare tale Bruni per la risoluzione di un contenzioso.

4. Velia a IT, lettera ms., Lamporecchio, 18 dicembre 1929, 2 cc. Velia informa il padre della avvenuta consegna delle carte del ricorso al Sig. Mancini, pronto a occuparsi della questione e a 
raccogliere delle firme per raggiungere lo scopo. Il Bruni ha lasciato i documenti richiesti e l'incarico al figlio.

5. Velia a IT, lettera ms., Lamporecchio, 15 gennaio 1930, 1 c. Velia riferisce a IT di aver mandato le lettere che gli aveva chiesto di inviare e che nessuno era venuto a richiedere i Calendari (il Calendario Agricolo Fascista, anno 1930).

6. Indirizzo del Marchese Perrone Compagni, carta ms., Firenze, s.d., 2 cc.

7. Certificato medico rilasciato a Idalberta, carta stampata e ms., Galluzzo, 28 aprile 1929, 1 c.

8. Busta di lettera proveniente dal Ministero per l'Economia Nazionale, Roma, 22 marzo 1929. Manca il contenuto.

9. Ilio Mancini a IT, lettera ms., Lamporecchio, 4 dicembre 1929, $2 \mathrm{cc}$. Mancini ringrazia IT per averlo raccomandato nell'assegnamento di una pensione di guerra e lo sostiene nella sua iniziativa a favore dell'erezione a Lamporecchio di un monumento dedicato ai Caduti della Grande Guerra.

10. Cammilli a IT, lettera ms., s.l. [Lamporecchio], 1 dicembre 1929, 2 cc. Il mittente descrive sinteticamente un progetto di costruzione di una Fontana monumento.

11. IT ad Argante, lettera datt., Firenze, 16 aprile 1930, 2 cc. IT descrive al destinatario le sue precarie condizioni di salute e gli esprime la volontà di parlargli di certe faccende a tu per tu.

12. Elenco dei Segretari Comunali dei Sindacati Fascisti dell'Agricoltura della Provincia di Firenze, foglio a protocollo ms., s.l. [Firenze], s.d. [post 1923], 2 cc. Elenco di 49 nomi.

13. IT ad Afrisio Vannacci, minuta ms., San Baronto, 11 agosto 1927, 4 cc. IT si lamenta del suo rapporto problematico con la moglie Amelia, sorella del destinatario, alla quale rimprovera il fatto di non aver voluto accettare il trasferimento a Firenze dove avrebbe potuto guadagnare di più e provvedere più adeguatamente alle cure dei familiari. Si lamenta poi di Torello, accusato di maltrattare Velia.

14. Appunto con indirizzo di Amerigo e Ada Lanconi, carta ms., s.l., s.d., 1 c.

15. Carta con vari appunti, carta datt. e ms., Lamporecchio, novembre 1929,1 c. Sul recto è presente una parte della lettera di Bruni Alessandro e Ancillotti Leopoldo che chiedono a IT dove si trovino le deliberazioni di Consiglio e di Giunta attestanti la loro assunzione in servizio per l'azienda Annonaria di Lamporecchio. A p. 2 v'è un datt. riportante un calendario delle fiere in programma in alcuni comuni della Provincia di Firenze. 
16. Copie di lettere di IT a vari destinatari, foglio a protocollo ms., [Firenze], 19-20 gennaio 1930, 2 cc. A p. 1 (19 gennaio 1930), IT offre all' ignoto destinatario la possibilità di costituire una Cooperativa Edile di cui IT stesso sarebbe dovuto essere Direttore. A p. 2 (19 gennaio 1930), IT informa la figlia circa il suo precario stato di salute, le chiede di inviare 50 copie del libro [Calendario Agricolo Fascista] al Martelli. A p. 3 (20 gennaio 1930), riferisce a tale Abramo di aver parlato coi sigg. Bandinelli e Puliti e che per primavera sarebbero stati disposti ad assumere nuovi operai. Allega lettera per la sorella Dusola, che riporta in copia a p. 4 in cui la invita a non impicciarsi dei fatti che non la riguardano.

17. IT a Ilio Mancini, minuta ms., Firenze, 5 febbraio 1930, 1 c. IT avverte l'amico fascista Mancini di aver deciso di non prendere parte alla ricostruzione del Fascio di Lamporecchio.

18. IT all'on. Luigi Razza, Presidente della Confederazione dei Sindacati Fascisti dell'Agricoltura, minuta ms., Firenze, 19 marzo 1930,1 c. IT chiede all'on. Razza che gli venga saldato il credito di 8.000 lire avanzato nei confronti della Confederazione dei Sindacati Fascisti dell'Agricoltura. A p. 2: «Estratto dall'Ufficio dell'Unione del mio avere al 3 febbraio 1930 VIII $^{\circ} \gg$.

19. Benvenuto Bettarini a IT, lettera ms., Lamporecchio, 4 febbraio 1930, 2 cc. Bettarini scrive a IT per informarlo circa l'avvenuta raccolta firme tra i muratori della zona presumibilmente per la costituzione di una Cooperativa edilizia.

20. Corrispondenza di Renzo - Cartellina rossa contenente le lettere inviate a IT da Renzo, probabile figlio illegittimo di IT e nato da una terza relazione.

1. Renzo a IT, lettera datt., Bengasi, 24 luglio 1924, 2 cc. Renzo parla della sua vita sotto le armi in Libia.

2. Renzo a IT, lettera datt., Bengasi, 22 agosto 1924, 1 c.

3. Renzo a IT, lettera ms., Bengasi, 29 novembre 1924, 1 c. Renzo si congratula con IT per la sua riammissione quale Segretario dell'Agricoltura alle Corporazioni Fasciste. Racconta di essere stato tre giorni in ospedale per un incidente occorsogli al viso. Si rammarica per le cattive condizioni di salute di Idalberta.

4. Renzo a Sofia, lettera ms., Pistoia, 27 dicembre 1928, 2 cc. Renzo si rammarica per le cagionevoli condizioni di salute di IT e avverte che a breve sarebbe partito per un lungo ciclo di operazioni belliche.

5. Renzo a Nanni [IT], lettera ms., Barce, 22 giugno 1928, 1 c. Renzo dice di aver assistito a quattro esecuzioni capitali in una settimana e di esserne rimasto impressionato. 
6. Renzo a IT, lettera ms., Bengasi, 31 maggio 1924, 2 cc. Renzo informa che è arrivato da pochi giorni a Bengasi per svolgere servizio militare. L'autore descrive la città e la vita quotidiana.

7. Renzo a IT, Bengasi, lettera ms., 10 ottobre 1924, 2 cc. Renzo informa IT che è dispiaciuto di non aver ricevuto da oltre un mese sue notizie. A interrompere la solita vita militare è stata la visita dell'on. Cantalupo, Sottosegretario delle Colonie venuto a visitare la Cirenaica.

8. Renzo a IT, lettera ms., Bengasi, 31 ottobre 1924, 2 cc. Renzo invita IT a non far troppi pensieri sulla morte. Lo informa che la vita militare non lo sta tediando, che l'ha scelta volontariamente e spera che la salute di Idalberta si sia rimessa.

9. Renzo a IT, lettera ms., Bengasi, 13 giugno 1924, 1 c. Renzo informa che è stato ca mbiato di reparto. Rifiuta, inoltre, la raccomandazione di un deputato proposta da IT.

10. Renzo a Idalberta, lettera ms., Bengasi, 22 agosto 1924, 1 c. Renzo descrive il suo campo militare situato in riva al mare.

11. Renzo a IT, lettera ms., Barce, 14 dicembre 1928, 2 cc. Renzo esprime a IT il suo rammarico per non aver ricevuto la sua posta per circa due mesi.

12. Renzo a IT, lettera ms., Bengasi, 4 luglio 1924,1 c. Renzo esprime riconoscenza a IT per i preziosi consigli. Descrive operazioni belliche contro i ribelli e parla dell'omicidio Matteotti.

13. Renzo a IT, lettera ms., Barce, 7 luglio 1928, 1 c. Renzo dice a IT che fa caldo e spera che gli esami elementari di Idalberta vadano bene.

14. Renzo a IT, lettera ms., Barce, 1 giugno 1928, 1 c. Renzo informa IT circa il viaggio di otto giorni appena fatto in mare e di essersi opposto alla decisione dei suoi superiori di mandarlo nelle zone più lontane e disagiate della colonia.

15. Renzo a IT, lettera ms., Bengasi, 11 giugno 1925, 2 cc. Renzo rivolge a IT parole che fanno intuire che tra i due c'è molto di più che un semplice legame affettivo.

4. Cartella nera contenente documenti vari:

1. Lettera aperta dei Partiti Popolari riuniti nel Comune di Lamporecchio, opuscolo, Pistoia, Tip. A. Ciattini, s.d., 4 pp. Documento di propaganda politica in vista delle elezioni comunali del 22 luglio [?].

2. IT a S.E. On. Marchi, Segretario della Federazione Fascista della Provincia di Firenze, minuta ms., Lamporecchio, 1 gennaio 1926, 1 c. IT chiede a Marchi di intervenire su una questione che si sta pro- 
traendo da 18 mesi senza che si sia giunti a risultati, ovvero chiede di portare a termine un' inchiesta sul comportamento non corretto tenuto da alcuni membri del fascio locale.

3. IT al Segretario e ai componenti della Federazione Fascista Provinciale di Firenze, minuta ms., s.l. [Lamporecchio], s.d. [1926], 3 cc. IT, in qualità di rappresentante della Sezione Fascista di Lamporecchio, si rivolge alla Federazione Provinciale sulla questione del fascio locale di cui sopra.

4. Al Gufo, contribuente non moroso e all'eletta schiera dei noti bestioni, opera a stampa, Empoli, Tip. Popolare, maggio 1915, 4 pp. (cfr. 3.6.8.).

5. Preghiera funebre dedicata a Felice e Amelia Niccolai, manifesto a stampa, Tip. G. Flori \& C., Pistoia, 1901, 2 pp.

6. Certificato di nascita di Lida Chiappini, documento comunale, Lamporecchio, 21 giugno 1928, 1 c.

7. Partecipazione di matrimonio di Talino Talini e Vannina Vannacci, cartoncino stampato, Lamporecchio, 15 gennaio 1931, 1 c.

8. Appunti scritti in latino, carta ms., s.l., s.d., $1 \mathrm{c}$.

9. Taccuino con appunti vari, bozze di discorsi politici, minute ecc., taccuino ms., s.l. [Lamporecchio], s.d. [1914], 29 cc.

5. Cartella nera e verde contenente documenti vari:

1. Ai Signori proprietari del Comune di Lamporecchio, fascicolo ms., Firenze, 7 dicembre 1921, 7 cc. Fascicolo dettagliato destinato ai proprietari iscritti al fascio che non avevano partecipato alla riunione del 28 novembre 1921.

2. Se la Borghesia non si rivoltasse, carta ms., s.l. [Firenze o Lamporecchio], s.d. [1915?], 1 c. Bozza incompleta di discorso politico sulla borghesia.

3. Il nostro dovere d'oggi e di domani, carta ms., s.l. [Firenze o Lamporecchio], s.d. [1915?], 1 c. Bozza incompleta di discorso politico sull'ingresso italiano in guerra.

4. Elenco spese entrata-uscita, carta ms., s.l., s.d., 1 c. Elenco probabilmente inerente alle attività economiche di uno dei giornali diretti da IT.

5. Guido Carbonai, Direttore de «La Riscossa» a tutti i fasci di Firenze, circolare a stampa, Firenze, s.d., 1 c. Circolare che invita tutti i fasci della Provincia di Firenze a pagare la quota mensile del giornale che era l'organo ufficiale dei Fascio fiorentino.

6. Cartellina giallo senape: «Sindacato Braccianti Agricoli di Lamporecchio». Contiene i seguenti documenti:

1. Lettera ai Signori Tarabusi Gianni, Minghetti Al., Minghetti An., minuta ms., s.l. [Firenze], s.d. [1923-1930], 1 c. IT, in qualità di Segretario Provinciale dei Sindacati Fascisti Coloni e 
Braccianti, redarguisce alcuni proprietari terrieri di Lamporecchio perché non si erano presentati a un'adunanza indetta dai Sindacati Fascisti dell'Agricoltura.

2. Biglietto d'invito - Sindacato Braccianti Lamporecchio, circolare datt., Lamporecchio, 5 marzo 1926, 1 c., 8 copie. IT, in qualità di Segretario Provinciale della Corporazione dell'Agricoltura, si rivolge a tutti gli iscritti del Sindacato Braccianti di Lamporecchio.

3. Giacomino il gobbo (Storia vera), carta ms., s.l., s.d., 1 c. Si narrano le tristi vicende di tale Giacomino, nato da famiglia povera e disagiata.

4. Appunti con elenco di nomi e numero di opere, carta ms., s.l., s.d., 1 c. Elenco di nomi, a cui, rispettivamente, corrispondono un certo numero di opere.

5. $\quad N^{\circ}$ degli operai assegnati a ciascuno dei seguenti proprietari, carta ms., Lamporecchio, s.d. [1923-1930], 1 c. Elenco dei nomi di diciassette proprietari terrieri di Lamporecchio, a cui viene fatto corrispondere un certo numero di operai assegnati. Sul verso, bozza di lettera da indirizzare a ognuno dei succitati proprietari.

6. Domande sospese e respinte dal Direttorio all'adunanza del $1^{\circ}$ gennaio 1926, carta ms., s.l. [Lamporecchio], s.d. [1926], 1 c. Elenco delle domande sospese e respinte dal Direttorio nell'adunanza del 1 gennaio 1926, nomina del nuovo Direttorio e, sul verso, elenco di nuove domande.

7. Lettere di richiesta d'ammissione al Sindacato Fascista Braccianti di Lamporecchio, lettere mss., Lamporecchio, 1925$1926,23 \mathrm{cc}$.

8. Circa l'adunanza dei proprietari terrieri di Lamporecchio con il Sindacato Fascista locale in merito all'equa ripartizione dei braccianti, carte mss. e dattiloscritte, Lamporecchio, febbraio 1925, 4 cc. Carte spillate con:

1. Foglio a protocollo ms. con «Elenco dei proprietari con 3 poderi ed altri ai quali è stato inviato l'invito per l'adunanza del giorno 19 corrente» indetta dal Sindacato Fascista per l'Agricoltura di Lamporecchio per la equa ripartizione dei braccianti della zona.

2. Circolare datt. del Comune di Lamporecchio, datata 16 febbraio 1925, in cui si invitano i proprietari a partecipare alla suddetta adunanza.

3. Circolare ms. da IT, Firenze, 13 febbraio 1925, in cui l'autore, in qualità di Segretario Provinciale dei Sindacati Coloni e Braccianti, invita i proprietari a partecipare alla suddetta adunanza. 
9. IT a Talini sui proprietari di Mastromarco rimasti fuori dalla distribuzione dei braccianti, minuta ms., s.l. [Lamporecchio], s.d. [1925-1926], 1 c. Si fa presente che alcuni proprietari della frazione di Mastromarco, puntualmente elencati, sono rimasti fuori dalla equa distribuzione dei braccianti.

10. Elenco di poderi con relative quote orarie da corrispondere agli operai, carta ms., Lamporecchio, 192[5-1926], 1 c. Presente elenco di fattorie con relative quote orarie da corrispondere agli operai

11. Federazione Provinciale dei Sindacati Fascisti - Atto Costitutivo Sindacato Braccianti di Lamporecchio, fascicolo ms., Lamporecchio, 2 febbraio 1923, 3 cc. Atto costitutivo del Sindacato Fascista dei Braccianti di Lamporecchio, di cui IT diventa Consigliere Esattore. All'atto è allegata una tabella con l'elenco degli iscritti a tale Sindacato.

7. Documenti inerenti al monumento ai Caduti di Lamporecchio

1. IT a Oreste Talini, minuta datt., Firenze, 15 settembre 1923, 3 cc. IT informa Oreste Talini di aver visto il bozzetto del monumento ai Caduti realizzato dallo scultore Cammilli di Firenze.

2. Verbale della Commissione esecutiva del Comitato pro monumento ai Caduti di Lamporecchio, carte dattiloscritte, Firenze, 16 ottobre 1923, 2 cc. La Commissione decide di affidare il lavoro al prof. Edoardo Cammilli e stabilisce le modalità della messa in opera.

3. Risposta di Cammilli al Comitato pro monumento ai Caduti di Lamporecchio, lettera datt., Firenze, 16 ottobre 1923, 1 c. Cammilli dice al Comitato che se questo non fosse riuscito a stanziare la cifra pattuita egli avrebbe realizzato un monumento di proporzioni più modeste.

4. IT a un avvocato, minuta datt., s.l., s.d., 1 c. Bozza incompleta di lettera.

5. IT a Giulio Rospigliosi, minuta ms., s.l., s.d. [1924], 1 c. IT nomina Giulio Rospigliosi quale membro del Comitato d'Onore per il monumento ai Caduti di Lamporecchio. Sul verso è presente un elenco di dieci nominativi a cui probabilmente è stata mandata la stessa nomina nel Comitato d'Onore.

6. IT a Edoardo Cammilli, minuta datt., Firenze, 8 ottobre 1923 , $2 \mathrm{cc}$. IT annuncia allo scultore Cammilli che il Comitato pro monumento ai Caduti di Lamporecchio ha scelto di affidargli la costruzione dell'opera monumentale in questione.

7. Risposta di Cammilli a IT, lettera datt., Firenze, 9 ottobre 1923, 2 cc. Lo scultore accetta l'incarico e si dice grato con il popolo di Lamporecchio. 
8. Oreste Talini a IT, lettera datt., Lamporecchio, 11 ottobre 1923, 1 c. Talini informa IT di aver sollecitato il fascio locale per la questione del monumento e che l'aspetto economico è lo scoglio da superare. Spera che l'On. Dino Philipson interceda presso la Principessa Rospigliosi per ottenere dei finanziamenti.

9. Oreste Talini a IT, lettera datt., Lamporecchio, 11 ottobre 1923, 2 cc. Talini informa IT sulla composizione demografica della frazione di Mastromarco in vista della richiesta fondi pro monumento. Nella seconda carta è presente un elenco recante i nominativi delle 79 famiglie residenti nella suddetta frazione.

10. Oreste Talini a IT, lettera ms., Lamporecchio, 11 ottobre 1923, $1 \mathrm{c}$. Piccola lettera, ms. in corsivo con inchiostro nero, inviata da Talini a IT per sollecitarlo a inoltrare delle suppliche all'On. [Dino Philipson] in merito alla questione del monumento, elemento di fondamentale importanza per la conquista elettorale della frazione di Mastromarco.

11. Oreste Talini a IT, lettera datt., Lamporecchio, 24 settembre 1924, 1 c. Talini informa IT circa la freddezza dimostrata da alcuni nei confronti dell'erezione del monumento ai Caduti. A tal proposito fu nominata una commissione esecutiva di soli fascisti. Inoltre, Talini sollecita IT a parlare in pubblico per convincere la popolazione a sottoscrivere in favore del suddetto monumento.

12. Bozza di convocazione alla Commissione per il monumento di Mastromarco, minuta ms., Lamporecchio, 192[3-1924], 1 c. Sul recto, annotazione dei membri componenti la Commissione di Mastromarco. Sul verso, elenco di nomi di padre, figlio e moglie, con relative generalità e a cui fa seguito un giudizio circa il loro orientamento politico.

13. Oreste Talini, Presidente del Comitato Pro M. Caduti, al Rag. Vincenzo Targioni, lettera datt., Lamporecchio, 2 agosto 1924, 1 c. Talini chiede assicurazioni al Rag. Targioni Vincenzo circa il rispetto della consegna dell'opera monumentale da parte di Cammilli, in quel momento impegnato in America.

14. Cammilli a IT, Firenze, 15 maggio 1924,1 c. Cammilli chiede a IT di risolvere con urgenza la questione del pagamento della prima trance del monumento.

15. IT al Sindaco di Lamporecchio circa la questione del pagamento a Cammilli, minuta ms., Firenze, 11 aprile 1924, 1 c. IT informa il sindaco Cesare Catolfi circa il pessimo trattamento che lo scultore Cammilli sta ricevendo da Talini, Presidente del Comitato pro monumento. 
16. IT al sindaco Catolfi, minuta datt., Firenze, 14 aprile 1924, 2 cc. Bella copia della lettera di IT a Catolfi.

17. Bozza di scritto polemico contro i detrattori del monumento, carte dattiloscritte, s.l. [Lamporecchio], s.d. [1923-4], 2 cc.

18. Cammilli a IT, lettera datt., Firenze, 18 aprile 1924,1 c. Cammilli avverte IT che il modello in gesso del monumento ai Caduti è pronto nel suo studio.

19. IT ad Amerigo Fedi, Sindaco di Larciano, minuta ms., Firenze, 16 febbraio 1923, 2 cc. IT comunica a Fedi di essere stato nello studio del prof. Cammilli per vedere lo stato d'avanzamento del monumento di Larciano.

20. IT al Cav. [Talini?], minuta ms., Firenze, 16 febbraio 1924, 3 cc. IT informa il Cavalier [Talini?] circa l'insoddisfazione di Cammilli sulla questione del mancato pagamento e lo sollecita a sbloccare la situazione. Propone, inoltre, una riscossione casa per casa dei contributi pro monumento e l'organizzazione di una fiera di beneficenza.

21. IT a Talini, Presidente del Comitato pro monumento ai Caduti, minuta ms., Lamporecchio, 21 aprile 1924, 2 cc. IT informa Talini circa la sua volontà di rassegnare le dimissioni da membro del Comitato pro monumento ai Caduti.

22. Elenchi con lista degli oblatori per [Fiera di Beneficenza o monumento ai Caduti?], carte mss., 5 cc. Elenchi di nominativi con relative quote versate per [Fiera di Beneficienza o Monumento ai Caduti?] e relativa quota della tassa di famiglia.

8. Ditta Talini e Morosi alla Società Elettrica del Valdarno, minuta datt., Lamporecchio, 23 luglio 1924, 1 c.

9. Attilio Martelli a IT, s.l. [Lamporecchio], s.d., 1 c. Martelli chiede a IT di occuparsi della disoccupazione del figlio andando a parlare con chi di dovere.

10. Nello Calzolai a IT, lettera ms., s.l. [Lamporecchio], s.d. [1923], 2 cc. Calzolai chiede a IT di combinare un incontro con l'on. Dino Philipson in merito alla linea elettrica Orbignano Porciano.

11. Nello Calzolai a IT, lettera ms., Lamporecchio, 22 agosto 1923, 2 cc. Sempre sull'argomento di cui sopra.

12. IT a Nello Calzolai, minuta datt., Lamporecchio, 22 settembre 1923, 1 c. IT riferisce a Calzolai in merito all' incontro avvenuto con l'on. Philipson. IT consiglia inoltre di preparare una bella accoglienza al deputato.

13. Nello Calzolai a IT, lettera ms., [Lamporecchio], s.d., 1 c. Calzolai riferisce che il Sindaco e altri consiglieri non sono lieti di accogliere l'on. Philipson.

14. Nello Calzolai a IT, lettera ms., Fornello, 12 novembre 1923, 2 cc. Sempre sulla questione di cui sopra. 
15. Mazzurri [?] a IT, lettera ms., Lamporecchio, 2 aprile 1926, 4 cc. Mazzurri [?] ribatte ad alcune accuse mosse da IT in merito alla sua condotta nel Sindacato fascista di Lamporecchio.

16. IT ad Amelia, minuta ms., Firenze, 192[3-1930], 1 c. IT informa la moglie dei suoi prossimi impegni e spostamenti.

17. Adunanza del Direttorio del Fascio di Combattimento coi proprietari del Comune di Lamporecchio, carte mss., Lamporecchio, 28 novembre 1921, 2 cc. Bozza di verbale di adunanza del Fascio di Lamporecchio in cui fu stabilito che le priorità erano il ristabilimento dell'ordine e della pace, la redistribuzione della manodopera tra gli operai e che il fascio locale dovesse essere l'organo moderatore tra braccianti e datori di lavoro.

18. Verbale di adunanza del Fascio di Lamporecchio circa l'avvio di una sottoscrizione per la costruzione della sede dell'Unione Politica Nazionale, foglio a protocollo ms., Lamporecchio, 6 ottobre 1921, 2 cc.

19. Bozza di Adunanza del Direttorio del Fascio locale sulla Fiera di Beneficienza, carta ms., Lamporecchio, 3 giugno 1922, 1 c. In quell'occasione si discusse in merito alla Fiera di Beneficienza da realizzare per raccogliere i fondi utili alla costruzione della sede dell'Unione Politica Nazionale. È presente un elenco di nominativi facenti parte di varie commissioni di frazione e la bozza della lettera che doveva essere inviata per la raccolta fondi.

20. Bozza di lettera da inviare a vari destinatari per la costruzione della sede dell'Unione Politica Nazionale, lettera ms., Lamporecchio, 1922, 1 c. Carta con la seguente intestazione: «Partito Nazionale Fascista - Sezione di Lamporecchio».

21. Elenco nominativi degl' individui ed enti ai quali si rivolgerà il Fascio per aver doni occorrenti alla Fiera di Beneficienza, carta ms., s.l. [Lamporecchio], s.d. [1922], 1 c. Elenco di nominativi di istituti, deputati e personalità a cui il Fascio di Lamporecchio si sarebbe dovuto rivolgere per aver doni occorrenti alla Fiera di Beneficienza organizzata in favore della costruzione della sede dell'“Unione Politica Nazionale".

22. Appunti del Verbale del Direttivo del Fascio di Lamporecchio in merito alla Fiera di beneficienza e al regolamento interno, carta ms., Lamporecchio, 29 giugno 192[2-1923], 1 c.

23. Bozza di lettera inviata da IT a vari destinatari circa il Concordato provinciale sull'agricoltura, carta ms., Lamporecchio, 12 aprile 1925,1 c. IT informa circa il contenuto del Concordato provinciale sull'agricoltura, che accordava l'indennità di caro-vita agli operai agricoli organizzati nei Sindacati Fascisti.

24. Alberto Volpi, AlNoto Uccello tristo e menzognero, manifesto a stampa, s.l., s.d., 2 pp. Componimento poetico di 15 strofe in ottava rima scritta da tale Alberto Volpi in polemica con IT. 
25. La risposta del Rosignuolo a suoi poco rispettabili avversari, manifesto a stampa, Pistoia, Tip. Cattini, s.d. [1912?], 2 pp. Componimento poetico di 44 strofe in ottava rima in cui IT risponde per le rime ai suoi detrattori e accusatori.

26. La questione politica ed economica secondo i conservatori ed i socialisti, manifesto a stampa, Pistoia, Tip. Cattini, s.d., 2 pp., 2 copie.

27. Al Gufo, contribuente non moroso, e all'eletta schiera dei noti bestioni, opera a stampa, Empoli, Tip. Popolare, maggio 1915, 4 pp. (cfr. 3.6.8 e 4.25.4).

28. «Il Risveglio della Plebe. Organo Socialista quindicinale dei comuni limitrofi al Mont'Albano», S. Baronto, 22 gennaio 1909, 4 pp. A p. 2, Lettera aperta all'Ill.mo sig. Olinto Catolfi Sindaco di Lamporecchio, scritta da IT.

29. Taccuino nero con appunti vari, taccuino ms., Lamporecchio, s.d. [1907], 32 cc. Bozze di scritti politici e anticlericali e appunti di vario genere (indirizzi, appuntamenti, calcoli, elenco spese e guadagni, promemoria).

6. Cartellina rossa: «Verbali Adunanze della C. E. e delle Assemblee. Anno $1921 \gg$. Presente timbro del Fascio di Combattimento - Sezione di Lamporecchio. Contiene:

1. Partito Nazionale Fascista - Fascio di Badia a Settimo al Segretario Politico del Fascio di Lamporecchio, foglio a protocollo a stampa, Signa, Tip. C. Caparrini, 11 settembre 1922, 1 c. Il Fascio di Badia a Settimo invita il Segretario Politico di Lamporecchio, IT, a partecipare alla cerimonia in suffragio dei Caduti fascisti.

2. Richieste di sottoscrizione al Sindacato Nazionale di Lamporecchio, minute mss., Lamporecchio, 26 marzo 1922, 9 cc. Richieste inoltrate dal sott. Fandi, Guido Nazzurri, Ermindo Bartolozzi, Alipio Giraldi, Alfredo Neri, Frizzolani Otello, Giuseppe Neri, Vittorio Bandini, Guido Marradi.

3. Antenore Minghetti a IT, lettera ms., Lamporecchio, 17 marzo 1922, 2 cc. Antenore Minghetti, replicando a un precedente sollecito di IT datato 14 marzo 1922, risponde che è impossibilitato ad assumere altri operai alle sue dipendenze.

4. Antenore Minghetti a IT, lettera ms., Lamporecchio 30 aprile 1922, $2 \mathrm{cc}$. Minghetti risponde ancora una volta di essere impossibilitato ad assumere altri operai alle sue dipendenze.

5. Santi Paganelli a IT, lettera ms., Lamporecchio, 19 marzo 1922, 2 cc. Santi Paganelli risponde a IT in merito alla questione della ricerca di occupazione per alcuni operai.

6. O. Donati, Sacerdote di Porciano, a IT, lettera ms., Porciano, 19 marzo 1922, 2 cc. Padre Donati scrive a IT per rispondere a una sua del 14 marzo 1922 in cui chiedeva di trovare occupazione ad alcuni operai. 
7. Ferruccio Giannini a IT, lettera ms., Fornello, 20 marzo 1922, 2 cc. Giannini risponde a IT sempre in merito alla questione dell'assunzione di alcuni operai.

8. Fratelli Marelli a IT, lettera ms., Lamporecchio, 19 marzo 1922, 1 c. I Fratelli Marelli rispondono sempre in merito alla questione dell'assunzione di alcuni operai.

9. Sacerdote L. Cioletti a IT, lettera ms., Lamporecchio, 20 marzo 1922, 1 c. Il Sacerdote Cioletti risponde a IT sempre in merito alla questione dell'assunzione di alcuni operai.

10. Sig.ra Torrigiani a Rodolfo, lettera ms., 22 marzo 1922, 2 cc. La Sig. ra Torrigiani risponde a tale Rodolfo sempre in merito alla questione dell'assunzione di alcuni operai.

11. Azelio Morosi a IT, lettera ms., Lamporecchio, 20 marzo 1922, 1 c. Morosi risponde a IT sempre in merito alla questione dell'assunzione di alcuni operai.

12. Elenco delle persone a cui IT ha inoltrato la richiesta di dare occupazione ad alcuni operai disoccupati, carta ms., s.l. [Lamporecchio], s.d. [1922], 1 c. Accanto a ogni nome c'è un numero che corrisponde al numero di operai potenzialmente assumibili.

13. Elenco dei proprietari per assumere al lavoro i disoccupati e riassunto delle loro risposte, carta ms., s.l. [Lamporecchio], s.d. [1922], 1 c. Elenco di nominativi a cui sono allegate le rispettive risposte in merito alla questione dell'assunzione di alcuni operai disoccupati di cui sopra.

14. Appunti - Case popolari e bozza di Verbale della Comm. Del Fascio degli industriali e proprietari della terra, Lamporecchio, s.d., $1 \mathrm{c}$.

15. Invito del Fascio di Marliana al Fascio di Lamporecchio per inaugurazione del Gagliardetto, carte stampate, Marliana, 9 settembre 1922, 2 cc.

16. Bozza di Verbale d'adunanza del Direttorio della Fed. Provinciale Fiorentina del PNF, carta ms., Firenze, 7 settembre 1922, 1 c.

17. IT alla Federazione Circondariale Fascista di Pistoia, minuta ms., Lamporecchio, 3 ottobre 1922, 3 cc. IT informa la Federazione Circondariale Fascista di Pistoia che in occasione dell'ultima assemblea dei soci si è proposto lo scioglimento del fascio stesso per «mancanza di attività fascista».

18. Verbali delle adunanze del Fascio di Lamporecchio, fascicolo ms., Lamporecchio, 6 ottobre 1921-17 aprile 1922, 14 cc.

19. Verbali delle adunanze del Fascio di Lamporecchio, fogli a protocollo mss., Lamporecchio, 1 luglio-17 ottobre 1922, 4 cc.

20. «La Bandiera del Popolo», Pistoia, 7 maggio 1922, 4 pp.

21. «La Bandiera del Popolo», Pistoia, 4 giugno 1922, 4 pp. 
7. «Corrispondenza», cartellina verde contenente i seguenti elementi:

1. «Note e appunti diversi», cartellina rosa con scritta a matita blu contenente i seguenti elementi:

1. «Domande da esaminare», parte di cartellina rossa contenente:

1. Domande di iscrizione al PNF di Lamporecchio, carte mss., Lamporecchio, 4 novembre 1922, 3 cc.

2. Ricevute di pagamento abbonamento a giornali fascisti, carte stampate e mss., Lamporecchio, 31 gennaio 1921-2 novembre $1922,7 \mathrm{cc}$.

2. Elenco documenti ricevuti dal Fascio di Combattimento di Lamporecchio, carta ms., Lamporecchio, 3 gennaio 1922, 1 c. IT attesta di aver ricevuto i documenti relativi all'amministrazione del Fascio di Lamporecchio di cui acclude un sintetico elenco: libro cassa, verbale adunanze, domande d'ammissione, imposte e competenze ecc.

3. Note di sottoscrizione in favore del Fascio di Lamporecchio, carta datt. e ms., Lamporecchio, s.d. [1921?], 1 c. Elenco di persone e relativa quota di sottoscrizione in favore del Fascio di Lamporecchio.

4. Sottoscrizione Pro Montenegro, fogli a protocollo mss., Lamporecchio, 5 gennaio 1922, 4 cc. Appello alla cittadinanza affinché sottoscrivesse in favore delle «Donne, vecchi, fanciulli che muoiono di fame» in Montenegro. Seguono elenchi di nominativi con relative quote di sottoscrizione.

5. Spese diverse, carte mss., Lamporecchio, $1922,3 \mathrm{cc}$. Elenco delle spese del PNF di Lamporecchio.

6. Relazione morale e finanziaria del Segretario Politico dall' istituzione del Fascio (nov. 1920) al 29 giugno 1922 e carte di verbali d'adunanza, carte mss., Lamporecchio, 28 giugno 1922, $5 \mathrm{cc}$. Resoconto dei primi venti mesi dell'attività del Fascio di Lamporecchio.

7. Registro delle somme incassate e pagate dal Fascio di Lamporecchio nel corso del primo semestre del 1922, fogli a protocollo mss., Lamporecchio, giugno 1922, 4 cc.

8. Nota delle spese sostenute dal Segretario Politico, carta ms., Lamporecchio, Ottobre 1922, 1 c. Annotazione delle spese sostenute e delle somme ricevute dal Segretario Politico del Fascio di Lamporecchio, IT, nel corso del 1922.

2. Registro Calzature Del Grosso - IT 1920-21:

1. Registrazione delle calzature consegnate al sig. Angelo Del Grosso con relativi importi e acconti ricevuti. Anno 1920, carte mss., Lamporecchio, 17 dicembre 1920, $8 \mathrm{cc}$.

3. Lettere di dimissioni dal Fascio di Lamporecchio, lettere mss. + tessere a stampa, Lamporecchio, 15-16 ottobre 1922, 14 cc. Alle lette- 
re di dimissioni sono allegate le tessere del PNF di Lamporecchio dell'anno 1922 appartenute a Primo Bagnoli e Adolfo Venzi.

4. Federazione Fascista Fiorentina. Appello a tutti i Segretari dei Fasci e del Sindacati Nazionali della Provincia, opuscolo, Firenze, 10 agosto 1922, 2 cc. Il Segretario Regionale della Federazione Fiorentina dei Sindacati Nazionali Fascisti, Persindo Giacomelli, il Segretario Federale della Federazione Fascista Fiorentina, Carlo Romagnoli, e il Segretario Provinciale della Corporazione Fiorentina dei Sindacati Nazionali Fascisti, IT, fanno propaganda a favore dei Sindacati Fascisti e invitano «contadini, braccianti e ogni sorta d'operai manuali» ad acquistare un nuovo giornale che avrebbe dovuto prendere vita di lì a poco.

5. PNF di Marliana al PNF di Lamporecchio, raccomandata datt., Marliana, 23 maggio 1922, 2 cc. Invito di partecipazione all'inaugurazione del Gagliardetto della squadra "Alessandro Zanni”.

6. PNF di Bassa al Direttorio del Fascio di Lamporecchio, lettera ms., Bassa, [1922], 2 cc. Invito di partecipazione all'inaugurazione del gagliardetto della sezione locale. Tra gli interventi oratori in programma era previsto anche il contributo di IT.

7. Unione Artistica Italiana al PNF di Lamporecchio, lettera a stampa, Firenze, [1921-1922], 1 c. Pubblicità per la vendita di un quadro allegorico in ricordo del Milite Ignoto.

8. PNF di S. Miniato al Direttorio del Fascio di Lamporecchio, lettera a stampa, S. Miniato, 30 maggio 1922, 1 c. Invito di partecipazione alla cerimonia di commemorazione del fascista Umberto Pontanari, caduto il 14 maggio 1921.

9. PNF di S. Angelo a Lecore al Segretario politico del Fascio di Lamporecchio, lettera datt., S. Angelo a Lecore, 19 aprile 1922, 1 c. Invito di partecipazione a una lotteria per raccogliere fondi in favore della realizzazione del Gagliardetto del fascio di S. Angelo a Lecore.

10. PNF di Stabbia al Direttorio del Fascio di Lamporecchio, lettera datt., Stabbia, 27 maggio 1922, 1 c. Invito di partecipazione all'inaugurazione del gagliardetto del fascio di Stabbia.

11. Adunata Camice nere della Toscana, volantino a stampa, Firenze, 23 maggio 1922, 1 c. Si invitano i fasci della Toscana a radunarsi a Firenze il 28 maggio 1922 per inaugurare il Labaro della Fed. Provinciale.

12. PNF di Livorno al Direttorio del Fascio di Lamporecchio, lettera a stampa, Livorno, 18 maggio 1922, 2 cc. Invito di partecipazione a un'adunata commemorativa in ricordo del giovane fascista Giorgio Moriani, ucciso un anno prima. Al comizio, fissato per il 1 giugno, avrebbero dovuto prendere la parola Costanzo Ciano, Roberto Farinacci e Dino Perrone Compagni. 
13. PNF di San Piero a Ponti al Direttorio del Fascio di Lamporecchio, lettera a stampa, S. Piero a Ponti, 25 maggio 1922, 2 cc. Invito di partecipazione, in data 1 giugno 1922, all' inaugurazione del gagliardetto del fascio di San Piero a Ponti.

14. PNF - Federazione Provinciale Fiorentina: Per la creazione di un organismo provinciale delle Corporazioni Sindacali, volantino a stampa, Firenze, maggio 1922, 1 c.

15. PNF di Stabbia al Fascio di Lamporecchio, lettera ms., Stabbia, 1 giugno 1923,1 c. Si informa che l'inaugurazione è stata sospesa fino a nuovo ordine.

16. Telegramma di Mazzoni a IT, telegramma, Castelfiorentino, 15 aprile 1922, $1 \mathrm{c}$.

17. Elenco di deliberazioni e di questioni da discutere nel Fascio di Lamporecchio, carte mss., s.l. [Lamporecchio], s.d. [1921-1922], 2 cc. Elenco in nove punti di questioni deliberate o da deliberarsi in sede di Consiglio direttivo all'interno del Fascio di Lamporecchio.

18. Bozza di O.d.g. dell'Assemblea direttiva del Direttorio del Fascio, minuta ms., Lamporecchio, 29 marzo 1922, 1 c. Sul recto, incipit incompleto di lettera al Segretario Politico di Montale, datata 27 marzo 1922. Sulla prima metà del verso, è presente la seguente dicitura, «Accettati dopo chiarimenti e richiamo», seguita da un elenco di nominativi, alcuni dei quali cancellati con segno di spunta. Nella metà inferiore del verso, «Ordine del giorno dell'Ad del Direttorio del 29 marzo 1922», con elenco in undici punti di questioni da discutere.

19. Bozza di O.d.g. dell'Assemblea direttiva del Direttorio del Fascio, carta ms., Lamporecchio, 6 ottobre 1921, 1 c. Elenco di questioni discusse nel suddetto O.d.g. Nella Commissione delegata alla raccolta fondi per la costruzione della sede nel fascio locale presente anche il nome di IT.

20. Bozza di O.d.g. dell'Assemblea direttiva del Direttorio del Fascio, carta ms., Lamporecchio, 31 dicembre 1921, 1 c. Vengono esaminate le domande di ammissione di alcuni aspiranti fascisti locali.

21. Bozza di O.d.g. dell'Assemblea direttiva del Direttorio del Fascio, carta ms., Lamporecchio, 8 febbraio 1922, 1 c. Vengono esaminate le domande di ammissione di alcuni aspiranti fascisti locali e discusse altre questioni.

22. Fasci Italiani di Combattimento di Vinci al Fascio di Lamporecchio, lettera ms., Vinci, 2 aprile 1922, 1 c. Invito di partecipazione, in data 9 aprile 1922, alla cerimonia d'intitolazione della Fiamma della squadra di Vinci al nome del fascista pratese Federigo Guglielmo Florio.

23. Bozza di verbale dell'Assemblea del Direttorio fascista della Cerbaia - Pagamento quote, carta ms., Lamporecchio, 16 aprile 1922, 1 c. 
Oggetto del verbale: pagamento delle quote d'iscrizione. Sul verso, presente elenco di nominativi.

24. Bozza di verbale dell'Assemblea del Direttorio del Fascio di Lamporecchio, carte mss., Lamporecchio, 19 gennaio 1922, 2 cc. Oggetto di discussione all'ordine del giorno: regolamento di conti con «Il Popolo d'Italia» e ammissione di nuovi soci. È presente un elenco di nominativi affiancati dalle quote associative versate.

25. Bozze di due verbali del Consiglio Direttivo del Fascio di Lamporecchio, carte mss., Lamporecchio, 16 maggio 1921-4 giugno 1921, 2 cc. Nel primo, datato 16 maggio 1921, vengono accettate ventinove richieste di associazione al fascio locale e il presidente IT viene delegato per formulare «un regolamento interno circa la disciplina tra $\mathrm{i}$ componenti del fascio e relativo statuto». Nel secondo verbale, datato 4 giugno 1921, viene indetta un'adunanza con tutti i proprietari terrieri per deliberare circa la disoccupazione e a tal proposito viene nominata una Commissione composta da alcuni dei proprietari stessi in rappresentanza delle varie frazioni di Lamporecchio.

26. Bozza di verbale del Consiglio Direttivo del Fascio di Lamporecchio, carte mss., Lamporecchio, 4 gennaio 1922, 1 c. All'O.d.g.: luce elettrica e avv. Rozzi (pagamenti), tessere 1922, soprattassa ai soci abbienti, lettere e manifesti, ammissione di nuovi soci.

27. Bozze di verbali del Consiglio Direttivo del Fascio di Lamporecchio, carte mss., Lamporecchio, 6 giugno- 6 ottobre 1921, 3 cc. Nel primo, datato 6 giugno 1921, si discute della questione della disoccupazione tra gli operai agricoli della zona. Si stabilisce che i proprietari potranno procedere alle assunzioni solo tramite il fascio locale e che la deliberazione presente sia pubblicata su «L'Alleanza». A tal proposito IT riferisce in merito allo stato finanziario del giornale da lui diretto, bisognoso di nuovi fondi: viene deliberato all'unanimità di continuare le pubblicazioni e di aprire una pubblica sottoscrizione nel paese. Nella seconda bozza di verbale, datata 6 ottobre 1921, si mettono agli atti le dimissioni del Segretario politico del fascio, Ezio Galeotti, e la nomina di Tosi Iginio.

28. Comunicato del PNF - Federazione Circondariale di Pistoia alla sezione di Lamporecchio, carta datt., Pistoia, 24 luglio 1922, 1 c. Si invita la sezione di Lamporecchio a contribuire alla diffusione di un nuovo «giornale di battaglia» del Circondario Pistoiese da usare come arma di lotta «contro la stampa antipatriottica e sovversiva».

29. Comunicato del PNF - Federazione Circondariale di Pistoia alla sezione di Lamporecchio: Statuto della Fed. Circ. Fascista di Pistoia, carte dattiloscritte, Pistoia, 9 giugno 1922, 4 cc.

30. Domanda di ammissione all'Unione Cooperativa Agricola di Consumo, carta stampata e ms., Lamporecchio, 20 novembre 1920, 1 c. I 
coniugi Cioli chiedono l'ammissione all'Unione Cooperativa Agricola di Consumo, di cui IT era, probabilmente, il Presidente.

31. PNF di Tizzana al fascio di Lamporecchio, carta datt., Quarrata, 15 aprile 1922, 1 c. Il Segretario politico del PNF di Tizzana, Vannino Vannini, invita il fascio di Lamporecchio a partecipare, in data 30 aprile 1922, all' inaugurazione della locale squadra d'azione, intitolata a Guglielmo Federigo Florio.

32. Richiesta informazioni su Innocenti Gino.

1. PNF di Sansepolcro al PNF di Pistoia, lettera ms., Sansepolcro, 10 giugno 1922, 1 c. Il PNF di Sansepolcro chiede informazioni su Innocenti Gino, nato a Lamporecchio il 24 febbraio 1899 , carabiniere a Sansepolcro e richiedente iscrizione al locale fascio.

2. PNF di Pistoia al PNF di Lamporecchio, lettera ms., Pistoia, 12 giugno 1922, 1 c. Il PNF di Pistoia riferisce al PNF di Lamporecchio quanto richiesto dal PNF di Sansepolcro.

33. La Gioventù Italica che vigila il sacro Tempio dell'Italia vittoriosa, cartolina postale, Firenze, s.d., 1 c. Sul verso si legge che la cartolina è stata pubblicata «a Beneficio dell'Asilo Orfani di Guerra "Vittorio Veneto" in Firenze».

34. Comitato per il monumento ai Caduti in guerra - Fucecchio, carte stampate, 14 giugno 1922, 2 cc. Invito di partecipazione all'inaugurazione, in data 25 giugno 1922, del locale monumento realizzato da Augusto Miniati. Nell'allegato programma della giornata si legge che era previsto l'intervento oratorio di Carlo Delcroix.

35. «La Frusta. Giornale di propaganda fascista»- bilancio delle spese, carta datt., s.l., s.d., 1 c. Elenco delle spese e degli introiti ottenuti in favore de «La Frusta», giornale di propaganda fascista di Pistoia.

36. PNF di Montale al fascio di Lamporecchio - Richiesta contributo pro monumento ai Caduti, lettera a stampa, 27 giugno 1922, 1 c. Richiesta di contributo per il monumento ai Caduti in guerra di Montale.

37. Cassa di Risparmio di Pistoia al Fascio di Combattimento di Lamporecchio, lettera datt., Pistoia, 2 luglio 1922, 1 c. La Cassa di Risparmio di Pistoia informa il Fascio di Combattimento di Lamporecchio che l'utile del bilancio 1921 era già stato distribuito.

38. PNF di Albenga al fascio di Lamporecchio, lettera a stampa, Albenga, 13 luglio 1922, 1 c. Il fascio di Albenga invita il fascio di Lamporecchio a versare un contributo per la costituzione di un Gagliardetto.

39. PNF di Palaia al fascio di Lamporecchio, lettera a stampa, Palaia, 12 giugno 1922, 1 c. Invito di partecipazione all'inaugurazione del gagliardetto del fascio di Palaia, previsto per il 18 giugno 1922. L'evento fu però rimandato. 
40. PNF di Empoli - Gruppo Femminile al fascio di Lamporecchio, lettera a stampa, Empoli, 5 aprile 1922, 1 c. La Segretaria del Gruppo femminile del PNF di Empoli, Teresa Pagliai, invita il fascio di Lamporecchio a dare un contributo per la costruzione di una cappella mortuaria in favore delle nove vittime della strage di Empoli del 1 marzo 1921.

41. Luigi Pancani a IT, lettera ms., 24 marzo 1922, 2 cc. L'autore, un proprietario terriero, scrive a IT in merito alla questione della collocazione di alcuni coloni disoccupati nei suoi poderi.

42. PNF di San Minato al fascio di Lamporecchio, lettera a stampa, San Miniato, marzo 1922, 1 c. Invito di partecipazione alla commemorazione di Luigi Pontecchi, fascista fiorentino morto il 3 aprile 1921 e a cui il fascio di San Miniato aveva intitolato il proprio gagliardetto.

43. PNF Federazione Provinciale Fiorentina al Fascio di Lamporecchio - Manifestazione Fascista 28 maggio 1922, Firenze, 1922, 2 cc. Si invitano tutti i fasci della regione a partecipare a una grande manifestazione fascista, prevista per il 28 maggio 1922 a Firenze.

44. PNF di Tizzana al fascio di Lamporecchio, lettera a stampa, Quarrata, 24 marzo 1922, 2 cc. Il Segretario Politico del PNF di Tizzana, Ten. Donatello Bracali, chiede di inviare una quarantina di uomini per imporre la chiusura di un circolo operaio considerato sovversivo e per lì costituirvi la nuova sede del fascio locale.

45. Deputato al PNF di Lamporecchio, lettera datt., Firenze, 27 febbraio 1922, 2 cc. L'autore informa il fascio di Lamporecchio che il Ministero dei Lavori Pubblici gli ha fatto pervenire una risposta in merito ai lavori per la strada di Giugnano.

46. IT al compagno fascista Venzi e programma della Manifestazione regionale fascista, minuta ms. e carte stampate, Firenze, 25 maggio 1922,3 cc. IT, in qualità di Segretario politico del fascio di Lamporecchio, dà disposizioni al compagno di partito Venzi circa l'organizzazione dell'adunata regionale fascista prevista per il 28 maggio 1922 a Firenze. Presenti due carte stampate con chiamata all'adunata firmata da Dino Perrone Compagni e programma dettagliato della suddetta adunata.

47. PNF di Serre di Rapolano al fascio di Lamporecchio, lettere a stampa, 11 maggio 1922, 1 c. Richiesta di contributo al fascio di Lamporecchio per ultimare l'acquisto della sede del fascio locale.

48. PNF di Monsummano al fascio di Lamporecchio, lettere datt., Monsummano, 2 giugno 1922, 1 c. Si avverte che la cerimonia che avrebbe dovuto aver luogo a Monsummano è stata rimandata a causa delle disposizioni governative che vietano tutte le manifestazioni pubbliche.

49. Fasci Autonomi Toscani al fascio di Lamporecchio, lettera a stampa, Firenze, 5 aprile 1922, 1 c. L'incaricato dei F.A.T. chiede al destina- 
tario una «precisa risposta dopo che l'assemblea sarà portata a conoscenza del contenuto della circolare e del manifesto».

50. PNF Federazione Provinciale Fiorentina al fascio di Lamporecchio, lettera datt., Firenze, s.d. [post 28 maggio 1922], 1 c. Invito di partecipazione alla proiezione del film della «grande Adunata Regionale Toscana».

51. PNF Unione Mandamentale di Pontassieve alla sezione di Lamporecchio, lettera a stampa, Pontassieve, s.d. [1922], 1 c. Invito di partecipazione all'inaugurazione dei gagliardetti di alcune sezioni aderenti.

52. PNF di S. Miniato alla sezione di Lamporecchio, lettera ms., San Miniato, 2 giugno 1922, 1 c. Si annuncia che la «Festa annunciata dalla precedente circolare è stata rimessa, causa ordine della Pubblica Sicurezza».

53. PNF di Pistoia alle sezioni di San Baronto e di Lamporecchio, lettera a stampa, Pistoia, 15 aprile 1922, 2 cc., 2 copie. Invito di partecipazione alla commemorazione, prevista per il 7 maggio 1922, «del compianto fascista della prima ora Pacino Pacini di Pistoia, studente di terza liceale, barbaramente trucidato il 1 maggio 1921 presso la stazione di Viareggio».

54. «La Riscossa» - Società Anonima Fiorentina Fascista Editrice al fascio di Lamporecchio.

1. Lettera datt., Firenze, 29 aprile 1922, 1 c. Il Segretario Provinciale del PNF, Carlo Romagnoli, e il Direttore de «La Riscossa», Guido Carbonai, annunciano la costituzione di una Società Anonima Fiorentina Fascista Editrice, concepita per assicurare la continuità delle pubblicazioni de «La Riscossa». A tal proposito fanno richiesta al fascio di Lamporecchio affinché faccia una sottoscrizione al suddetto ente.

2. Lettera a stampa, Firenze, 2 maggio 1922, 2 cc. Il Comitato promotore del S.A.F.F.E. annuncia la costituzione della società, nata con lo «scopo principale di pubblicare e gestire il giornale settimanale «La Riscossa» e subordinatamente di curare la pubblicazione di libri ed opuscoli che riflettano e aiutino il movimento e l'idea fascista...». Presente allegato da compilare per la sottoscrizione di 100 Lire in favore della suddetta società.

55. PNF di Santa Croce sull'Arno al fascio di Lamporecchio, lettera datt., Santa Croce sull'Arno, [1922], 1 c. Invito di partecipazione all'inaugurazione del Gagliardetto dell'Avanguardia di Santa Croce sull'Arno.

56. PNF di Firenze al fascio di Lamporecchio, lettera datt., Firenze, 18 luglio 1922, 1 c. Il Segretario del Fascio di Firenze, G. Valeriani, in- 
forma il fascio di Lamporecchio di non poter contribuire in alcun modo alla costruzione della loro nuova casa del fascio.

57. PNF di Vinci al Fascio di Lamporecchio, raccomandata ms., Vinci, 8 aprile 1922, 1 c. Richiesta di contributo per l'acquisto di biglietti della lotteria organizzata in favore del fascio locale.

58. PNF di Tizzana al fascio di Lamporecchio, lettera ms., Tizzana, 7 aprile 1922, 1 c. Invito di partecipazione al comizio del maestro Martini sul tentativo di trasformare il Circolo di Lucciano nella sede dei fascisti locali.

59. Federazione Circondariale Fascista del Pistoiese ai Segretari politici delle Sezioni fasciste del Circondario, raccomandata datt., [Pistoia], [1922], 1 c. Invito rivolto a tutti i Segretari politici delle Sezioni fasciste del Circondario per decidere una concorde strategia d'azione da adottare nei confronti degli oppositori politici. Si richiede inoltre di sottoscrivere in favore dei compagni fascisti reclusi in carcere.

60. Direttore dell'ufficio stampa de «La Riscossa $»$ al Direttore del giornale, lettera datt., Roma, 13 luglio 1922, 1 c. Il Direttore dell'ufficio stampa de «La Riscossa», Bastianini, scrive al Direttore del giornale, Carbonai, in merito al rischio di chiusura della testata.

61. PNF di Pistoia al Segretario politico del fascio di Lamporecchio, lettera datt., Pistoia, 5 ottobre 1922, 1 c. Il PNF di Pistoia invita i componenti del fascio di Lamporecchio a versare un contributo in favore dei sopravvissuti dello 'scoppio di Falconara' (il 28 settembre 1922 un fulmine colpì e fece esplodere una polveriera situata nel castello di Falconara, sovrastante S. Terenzo), in particolare per gli abitanti di S. Terenzo e Lerici.

62. PNF di Pistoia al Segretario politico del fascio di Lamporecchio, lettera datt., Pistoia, 5 ottobre 1922, 1 c. Invito di partecipazione all'adunata di camice nere prevista a Pistoia il 4 novembre 1922.

63. PNF - Gruppo Femminile di Pistoia: Nota di sottoscrizione a pro del gruppo femminile per il gagliardetto, carta datt. e a stampa, Pistoia, 12 ottobre $1922,1 \mathrm{c}$.

64. PNF - Gruppo Femminile di Pistoia al fascio di Lamporecchio, carta datt., Pistoia, 12 ottobre 1922, 1 c. Invito di partecipazione al corteo in camicia nera previsto per il 4 novembre 1922.

65. Comitato Pro danneggiati per lo scoppio della polveriera di Falconara, manifesto a stampa, Pistoia, 2 ottobre 1922, 1 c. Il suddetto comitato era stato costituito a Pistoia per raccogliere offerte in favore degli abitanti dei territori colpiti dalla terribile esplosione di Falconara. Gli enti costituenti di detto Comitato furono: Ass. Nazionale Mutilati e Invalidi di Guerra, PNF, Croce d'Oro, Confraternita della Misericordia, Ass. Nazionale Combattenti, Pubblica Assistenza. 
66. Comitato Pro danneggiati per lo scoppio della polveriera di Falconara al Segretario politico di Lamporecchio, lettera datt., Pistoia, 13 ottobre 1922, 1 c. Il suddetto Comitato chiede al fascio di Lamporecchio di rimettere la nota di sottoscrizione entro il 15 ottobre 1922.

67. Federazione Circondariale Fascista di Pistoia alle Sezioni Federate, lettera datt., Pistoia, 21 agosto 1922, 1 c. Il Direttorio della Federazione Circondariale Fascista di Pistoia (Spinelli e Lenzi) dà disposizioni alle Sezioni Federate circa alcune questioni: abbonamento al giornale «L'Azione fascista»; invito a far iscrivere nelle Corporazioni sindacali di mestiere «anche i fascisti che esercitano un mestiere qualunque»; indirizzo per la corrispondenza e numero di telefono della Federazione Circondariale.

68. Federazione Circondariale Fascista di Pistoia ai Comandi delle Squadre d'azione, Lettera datt., Pistoia, 23 agosto 1922, 1 c. L'Ispettore Circondariale delle Squadre ringrazia i comandanti e tutti gli squadristi per l'opera svolta «durante lo sciopero generale bolscevico».

69. Federazione Circondariale Fascista di Pistoia ai Segretari politici, lettera datt., Pistoia, 24 agosto 1922, 1 c. Si invitano tutti i Segretari politici a rispondere alla circolare del 21 agosto inerente corrispondenza, elenco abbonati all'«Azione Fascista», raccolta adesioni per le Corporazioni sindacali e coordinamento per il rafforzamento del Partito.

70. Telegramma a Torello Chiappini, telegramma ms., Correggio, 9 ottobre 1922,1 c. Il mittente fa un ordine di dieci fusti di vino a Torello Chiappini.

71. Luigi Pistolesi al Direttorio del PNF di Lamporecchio, lettera ms., Lamporecchio, 15 agosto 1922, 1 c. Pistolesi fa richiesta di iscrizione al PNF di Lamporecchio.

72. PNF di Lamporecchio - Quote mensili da riscuotere, carta ms., Lamporecchio, 192[2], 1 c. Appunti con i nominativi di coloro che dovevano versare ancora la quota di sottoscrizione al fascio di Lamporecchio.

73. Galeotti a IT, lettera ms., Lamporecchio, 27 ottobre 1922, 1 c. Il mittente informa IT circa la volontà di alcuni fascisti di annullare la nomina a medico condotto del Dottor Merlini, sospettato di essere socialista, in favore del Dottor Riccio di Rovigo, di fede fascista. L'autore smentisce l'estrazione socialista del Merlini, definendolo invece «italianissimo» e anche «propagandista al fronte».

74. Gruppo femminile del PNF di Pistoia al Direttorio del PNF di Lamporecchio, lettera datt., Pistoia, 12 ottobre 1922, 1 c. Richiesta di contributo.

75. Comitato di Redazione de «La Frusta» al fascio di Lamporecchio, lettera datt., s.l., s.d. [1922], 2 cc. Si pubblicizza l'uscita di un nuovo 
giornale della Federazione Circondariale Fascista del Pistoiese, «La Frusta. Giornale di propaganda fascista».

76. IT a Giulio [?], minuta ms., Firenze, 23 gennaio 1921, 1 c. IT, in qualità di Segretario Politico del Fascio di Lamporecchio e di Direttore della testata «L'Alleanza», chiede di indire un'adunanza «di tutti gli iscritti alla nostra Associazione compresi gli abbonati sostenitori $\gg$ per discutere alcune questioni.

77. IT a Venzi, minuta ms., Lamporecchio, 1922, 1 c. IT dà disposizioni al compagno di partito Venzi circa una pratica di pagamento e una di incasso per conto del partito. Lo incarica inoltre di inviare a otto potenziali iscritti al fascio locale una convocazione in sede per alcuni chiarimenti.

78. Risposte negative a domande di ammissione al fascio di Lamporecchio, minute mss., Lamporecchio, 19 gennaio 1922, 1 c. IT, in qualità di Segretario politico del PNF di Lamporecchio, risponde negativamente a domande di ammissione inoltrate al fascio locale.

79. IT a Adolfo [?], minuta ms., Firenze, 29 aprile 1922, 2 cc. IT informa il destinatario del fatto che l'adunanza tra braccianti e proprietari prevista per il 13 maggio sarebbe stata anticipata al 6.

80. IT alla Direzione de «Il Popolo d'Italia», minuta ms., Lamporecchio, 20 gennaio 1922, 1 c. IT riferisce di aver saldato il pagamento dell'abbonamento al giornale per conto del fascio di Lamporecchio.

81. PNF di Faltognano al Segretario politico del fascio di Lamporecchio, lettera ms., Faltognano (Vinci), 10 febbraio 1922, 1 c. Invito di partecipazione all' inaugurazione della sezione fascista di Faltognano.

82. PNF di Carmignano al fascio di Lamporecchio, lettera a stampa, Carmignano, 15 marzo 1922, 2 cc. Il Segretario del fascio di Carmignano, Morando Mari, inoltra l'invito a partecipare, il 2 aprile 1922 , alla commemorazione di due carabinieri assassinati il $28 \mathrm{Mar}-$ zo 1921.

83. Fascio di Larciano al fascio di Lamporecchio, lettera ms., Larciano, 1 marzo 1922, 1 c. I fascisti di Larciano chiedono a quelli di Lamporecchio di consegnargli una divisa delle squadre d'azione affinché il loro sarto di riferimento potesse ricavarne un modello per fare le divise agli squadristi larcianesi.

84. PNF di Montopoli al fascio di Lamporecchio, lettera a stampa, Montopoli, s.d. [1922], 1 c. Il PNF di Montopoli pubblicizza la vendita di una spilletta con su scritto «Occhio per occhio, dente per dente», dal cui ricavato il fascio montopolese ne avrebbe tratto finanziamento.

85. PNF di Certaldo al fascio di Lamporecchio, lettera datt., Certaldo, 9 marzo 1922, 2 cc. Invito di partecipazione a una cerimonia commemorativa in ricordo dell'eccidio di Certaldo del 28 febbraio 1921. 
86. Fascio di Cintolese al fascio di Lamporecchio, lettera ms., Cintolese, 17 gennaio 1922, 1 c. Invito di partecipazione, in data 29 gennaio 1922, all'inaugurazione del locale Gagliardetto, alla presenza di Ciano e dell'allora Segretario Provinciale Carlo Scorza.

87. Fascio di Santa Croce sull'Arno al fascio di Lamporecchio, lettera a stampa, S. Croce sull'Arno, 10 luglio 1921, 1 c. Invito a versare un contributo in favore dell'Ufficio del Segretario Politico Regionale per l'acquisto di un'automobile che potesse permettergli «di intervenire tempestivamente per cerimonie od altre urgenti necessità».

88. Fascio di Montopoli al fascio di Lamporecchio, lettera a stampa, Montopoli, 4 maggio 1921, 1 c. Invito a partecipare, in data 8 maggio 1922, alla cerimonia di inaugurazione del Gagliardetto.

89. Associazione Nazionale Combattenti di Firenze al fascio di Lamporecchio, lettera a stampa, Firenze, 3 maggio 1921, 1 c. Invito di partecipazione alla cerimonia di consegna della bandiera offerta dalle donne fiorentine alla sezione fiorentina dell'ANC.

90. Fascio di Vinci a quello di Lamporecchio, lettera ms., Vinci, 6 aprile 1922, 1 c. Il fascio di Vinci informa che l'inaugurazione della Fiamma della Squadra Guglielmo Florio è stata rimandata al 23 aprile.

91. PNF Fed. Provinciale Fiorentina al fascio di Lamporecchio, lettera datt., Firenze, 13 marzo 1922, 1 c. Si prega le sezioni fasciste della provincia a comunicare la data di costituzione dell'Avanguardia Giovanile e relativo numero di iscritti. Inoltre nel caso che l'Avanguardia debba ancora costituirsi s'invita le sezioni fasciste a sollecitarne la formazione.

92. Ispettore Circondariale del PNF di Pistoia al Comando circondariale delle Squadre d'azione, Pistoia, 25 marzo 1922, 1 c. Il neo Ispettore Circondariale del PNF di Pistoia, Ilio Lenzi, si rivolge ai Comandanti delle Squadre d'azione del Circondario pistoiese per ringraziarli dell' incarico ricevuto.

93. Fasci Italiani di Combattimento Comitato Centrale ai fasci di combattimento, circolare a stampa, s.l., s.d. [ottobre 1922], 1 c. Invito al raduno di Roma, nei giorni 21-24 ottobre 1922, in occasione del III Congresso Nazionale.

94. Fascio di Poggio a Caiano al fascio di Lamporecchio, lettera datt., Poggio a Caiano, 15 ottobre 1921, 1 c. Il Segretario politico del fascio di Poggio a Caiano, Giovanni Ciampi, sollecita la vendita di biglietti della lotteria in favore del loro fascio.

95. Federazione Provinciale Fiorentina del PNF ai Segretari politici della Provincia, lettera a stampa, Firenze, 14 ottobre 1921, 1 c. Il Segretario della Federazione Provinciale Fiorentina del PNF, Carlo Romagnoli, scrive di essere rammaricato del fatto che all'ultima riunione federale avevano partecipato solo 17 su 68 segretari politici. 
96. Fascio di Certaldo al fascio di Lamporecchio, lettera datt., Certaldo, 11 aprile 1921, 1 c. Invito di partecipazione alla cerimonia di inaugurazione del Gagliardetto della sezione di Certaldo.

97. Fascio di Pistoia al fascio di Lamporecchio, lettera ms., Pistoia, 28 aprile 1921,1 c. Si informa che il 30 aprile si sarebbe svolta un'adunanza dei segretari presso la casa del Marchese Dino Perrone Compagni.

98. Fascio di Pistoia al fascio di Lamporecchio, lettera datt., Pistoia, 4 maggio 1921, 1 c. Il Segretario del fascio di Pistoia, Maggiore Nesi, invita il fascio di Lamporecchio all'inaugurazione del Gagliardetto della sezione.

99. Fascio di Fucecchio al fascio di Lamporecchio, lettera datt., Fucecchio, 1 settembre 1922, 1 c. Invito a versare un contributo all'ex Tenente fascista Bagnoli Gaetano, vittima di una truffa.

100. Segreteria generale del PNF ai fasci locali, lettera a stampa, Milano, 31 dicembre 1921, 2 cc. Richiesta di compilazione di moduli inerenti le attività dei fasci.

101. Federazione Provinciale Fiorentina del PNF al fascio di Lamporecchio, Firenze, 10 gennaio 1922, 1 c. Il Segretario federale, Carlo Romagnoli, invita il fascio di Lamporecchio a consegnare l'elenco di tutti i soci comprensivo dei seguenti dati: «Nome e cognome - Paternità - Data di nascita - Luogo di nascita - Mestiere o professione - Luogo di abitazione attuale - Data di iscrizione al fascio».

102. Giuseppe Bregonzio al fascio di Lamporecchio, Milano, 29 aprile 1921, 1 c. La ditta di incisioni, smalti e miniature di Giuseppe Bregonzio promuove la vendita del distintivo sociale, di cui allega un campione gratuito.

103. Fascio di Vitolini al fascio di Lamporecchio, lettera ms., Vitolini, 9 maggio 1921, 1 c. Invito di partecipazione all'inaugurazione del Gagliardetto locale, occasione in cui avrebbe preso la parola anche IT.

104. Comitato elettorale dei Fasci di Combattimento di Firenze e Provincia al fascio di Lamporecchio, lettera datt., Firenze, 3 maggio 1921, 1 c. Si invita il fascio di Lamporecchio a fare esclusivamente «campagne per i propri candidati».

105. Fascio di Vinci al fascio di Lamporecchio, lettera datt., Vinci, 21 giugno 1921, 1 c. Invito di partecipazione all'inaugurazione del Gagliardetto. Nel programma della cerimonia era previsto anche un intervento di IT.

106. Fascio di Cerreto Guidi al fascio di Lamporecchio, lettera datt., 23 agosto 1922, 1 c. Invito di partecipazione alla Fiera di Beneficienza per l'erigendo monumento ai Caduti in Guerra di Cerreto Guidi.

107. Fascio di Castelfiorentino a IT, lettera datt., Castelfiorentino, 4 aprile 1922, 1 c. Invito di partecipazione alla cerimonia d'inaugurazione del fascio di Castelnuovo d'Elsa. 
108. Fascio di San Piero a Ponti al fascio di Lamporecchio, lettera a stampa, S. Piero a Ponti, 5 aprile 1922, 2 cc. Invito di partecipazione all'inaugurazione del Gagliardetto del fascio di San Piero a Ponti e di quello del fascio di Campi Bisenzio.

109. Fascio di Staffoli a quello di Lamporecchio, lettera a stampa, Staffoli, 27 settembre 1922, 1 c. Invito di partecipazione alla cerimonia d'inaugurazione del Gagliardetto e di una lapide dedicata alla memoria di Ferruccio Cristiani, «assassinato da mani immonde di comunisti».

110. Telegramma di Maggianti al fascio di Lamporecchio, telegramma ms., S. Croce sull'Arno, 30 settembre 1922, 1 c. Si informa che l'inaugurazione della Fiamma è stata rimandata al giorno 8 ottobre.

111. Comitato per le pubblicazioni a beneficio dell'asilo orfani di guerra "Vittorio Veneto" di Firenze al Segretario del fascio di Lamporecchio, lettera a stampa, Firenze, s.d., 1 c. Il Segretario del suddetto Comitato, Pasquale Aiazzi, invita il Segretario del fascio di Lamporecchio a diffondere presso i fascisti della propria sezione un'allegoria del Fascismo allegata in facsimile (ma non fisicamente presente).

112. PNF di Cerreto Guidi al fascio di Lamporecchio, lettera a stampa, Cerreto Guidi, 9 ottobre 1922, 2 cc. Invito di partecipazione alla cerimonia di commemorazione del Tenente Gino Pacini, primo segretario politico del fascio cerretese, «barbaramente assassinato a Poggio Tempesti dai social comunisti» la notte tra il 15 e il 16 ottobre 1921.

113. Comitato per le pubblicazioni a beneficio dell'Asilo Orfani di Guerra "Vittorio Veneto" di Firenze al Segretario del fascio di Lamporecchio, lettera a stampa, Firenze, s.d., 1 c. Si invita ad acquistare il «quadro rappresentante la gioventù eroica che vigila il sacro tempio dell'Italia Vittoriosa», dalle cui vendite il suddetto Comitato avrebbe tratto il profitto necessario al sostentamento dell'asilo.

114. PNF di Pescia al fascio di Lamporecchio, lettera a stampa, Pescia, 1 settembre 1922, 2 cc. Invito di partecipazione alla cerimonia d'inaugurazione del Gagliardetto d'intitolazione delle fiamme ai nomi di due fascisti defunti, Gastone Bartolini e Gustavo Mariani. Nel programma della giornata sono previsti gli interventi oratori di Dino Perrone Compagni, on. Costanzo Ciano, on. Dario Lupi, Carlo Scorza e del Magg. Cav. Evaristo Armani.

115. Direttore de «La Riscossa» al fascio di Lamporecchio, lettera datt., 24 luglio 1922, 1 c. Il Direttore de «La Riscossa», Guido Carbonai, annuncia di aver rassegnato le sue dimissioni date le gravi difficoltà economiche che stavano colpendo la testata da lui fondata.

116. ANC di Vinci al Segretario del fascio di Lamporecchio, lettera ms., Vinci, 3 settembre 1922, 1 c. Invito di partecipazione alla cerimonia 
d'inaugurazione della bandiera dell'associazione, realizzata dalle «gentili Donne vincesi».

117. Segretario politico di Lamporecchio, IT, a Benozzi Angiolo, minuta ms., Lamporecchio, s.d., 1 c. IT risponde al reclamo del Sig. Angiolo Benozzi circa la sua non ammissione al fascio di quella città.

118. Segretario politico di Lamporecchio, IT, a Alberto Fanti, minuta ms., Lamporecchio, s.d., 1 c. IT avverte il Sig. Alberto Fanti di rimborsare prima possibile 150 Lire all'Amministrazione del Fascio.

119. Spedizione di Porciano, carta ms., s.l., s.d., 1 c. Elenco di nominativi di persone che hanno partecipato alla «Spedizione di Porciano», così come recita l'iscrizione iniziale.

120. «Il Popolo d'Italia» al fascio di Lamporecchio, lettera a stampa, Milano, 1922, 1 c. «Il Popolo d'Italia» invita a chiudere il saldo a tutto il 31 dicembre 1921 entro e non oltre il 31 gennaio 1922.

121. Segretario Politico Regionale dei Fasci Italiani di Combattimento, Dino Perrone Compagni, al Segretario Politico del fascio di Lamporecchio, Firenze, 10 ottobre 1921, 1 c. Perrone Compagni si congratula con [IT] per la recente nomina a Segretario Politico del fascio di Lamporecchio.

122. Fascio Autonomo di Combattimento di Firenze, lettera a stampa, Firenze, s.d. [1922], 2 cc. Il Direttorio del Fascio di Firenze annuncia di costituirsi come ente autonomo rispetto al Comitato Centrale del Partito che, «compiendo un atto arbitrario e antistatuario, con lettera del 25 Marzo u. s. a firma Michele Bianchi, scioglieva il Fascio di Firenze con il pretesto che il Direttorio del Fascio non aveva ottemperato agli ordini ricevuti circa la espulsione del Ten. Tullio Tamburini e del Prof. Mario Pelegatti [...]».

123. Lettera del PNF di Palaia al fascio di Lamporecchio, lettera a stampa, Palaia, 30 agosto 1922, 2 cc. Invito di partecipazione, in data 10 settembre 1922, alla cerimonia di commemorazione del fascista Aldo Mazzei, ucciso il 4 settembre 1921, e all'inaugurazione dei Gagliardetti delle sezioni di Palaia e Partino. Erano previste inoltre le orazioni di Perrone Compagni, Borri, Cambellotti, Romagnoli e IT.

124. PNF di Cintolese al fascio di Lamporecchio, lettera a stampa, 10 luglio 1922, 1 c. Il PNF di Cintolese annuncia di aver costituito un Comitato per una Fiera di beneficenza volta a raccogliere fondi per aprire una biblioteca e per la previdenza sociale del piccolo paese. Per tale scopo si chiedono fondi per la riuscita dell'iniziativa.

125. Federazione Circondariale Fascista al fascio di Lamporecchio, lettera datt., Pistoia, 15 agosto 1922, 1 c. La Fed. Circondariale Fascista informa di aver mandato 15 copie del $2^{\circ}$ numero del settimanale «'Azione Fascista». 
126. PNF di Vitolini al fascio di Lamporecchio, lettera a stampa, Vitolini, 15 settembre 1922, 1 c. Il Segretario Politico del fascio di Vitolini, Santo Leporatti, invita il fascio di Lamporecchio a partecipare all'inaugurazione della locale Casa del Fascista.

127. Federazione Provinciale Fiorentina del PNF ai Fasci e Avanguardie della Provincia di Firenze, lettera a stampa, Firenze, s.d., 1 c. Il Segretario delle Avanguardie della Provincia di Firenze, Ricciardo Ricciardi Pollini, e il Segretario Federale, Carlo Romagnoli, chiedono a ogni Fascio della Provincia di comunicare se e da quanti elementi è composta l'Avanguardia locale e di costituirne una, qualora non fosse stata ancora costituita.

128. Fascio di Bardalone al PNF di Lamporecchio, lettera datt., Bardalone (San Marcello Pistoiese), 6 settembre 1922, 1 c. Invito di partecipazione alla cerimonia di inaugurazione del Gagliardetto del fascio di Bardalone.

129. PNF di S. Croce sull'Arno al fascio di Lamporecchio, lettera datt., S. Croce sull'Arno, 21 luglio 192[1-2], 1 c. Il Segretario Politico di S. Croce sull'Arno informa il fascio di Lamporecchio di non poter in alcun modo fare un'oblazione in loro favore.

130. Federazione Provinciale Fiorentina del PNF al Segretario del PNF di Lamporecchio, lettera a stampa, Firenze, s.d., 1 c. Si parla della volontà di istituire «un servizio di auto-trasporti per poter celermente portare dalle località di produzione ai centri di consumo le derrate alimentari in genere». Tale operazione, volta ad arginare il problema del caroviveri e delle speculazioni commerciali, aveva l'intento di far guadagnare al Partito fascista «moltissime simpatie e adesioni».

131. Federazione Provinciale Fiorentina del PNF a tutti i fasci della Provincia, circolare a stampa, Firenze, s.d., 1 c. Il Segretario della Federazione Provinciale fornisce alcune istruzioni a tutti i fasci della Provincia di Firenze.

132. PNF di San Casciano Val di Pesa al fascio di Lamporecchio, lettera ms., San Casciano Val di Pesa, s.d., 1 c. Richiesta di informazioni circa Ginanni Luigi, aspirante iscritto al Partito Fascista di San Casciano.

133. Ditta Renato Baldi \& C. Armi e Munizioni al fascio di Lamporecchio, lettera datt., Firenze, 14 settembre 1922, 1 c. La suddetta ditta pubblicizza i suoi prodotti al destinatario.

134. PNF di S. Angelo a Lecore (Poggio a Caiano), lettera a stampa, S. Angelo a Lecore, 25 settembre 1922, 1 c. Invito di partecipazione, in data 1 ottobre 1922, all'inaugurazione del Gagliardetto e della Fiamma del fascio di S. Angelo a Lecore.

135. «Fratelli Bertarelli. Fabbrica di Gagliardetti - Bandiere - Fiamme del Partito Nazionale Fascista», rivista pubblicitaria, Milano, n. 10, 25 agosto 1922, 4 pp. 
2. «Corrispondenza. Pezze d'appoggio e documenti giustificativi. Anno 1922», cartellina rossa contenente i seguenti elementi:

1. Richieste di adesione al PNF di Lamporecchio e altri documenti:

1. Prima richiesta scritta di Enrico Gaggioli, lettera ms., Lamporecchio, 3 novembre 1922, 1 c.

2. Seconda richiesta di Enrico Gaggioli, lettera ms., Lamporecchio, 3 novembre 1922, 1 c.

3. IT, Segretario politico del fascio di Lamporecchio, al Parroco di S. Baronto e Porciano, minuta ms., Lamporecchio, 31 ottobre 1922, 1 c. IT invita il Parroco di S. Baronto e Porciano «a rientrare nelle semplici funzioni di Sacerdote per l'esercizio del suo ministero spirituale; cioè dimettersi da qualunque Istituzione di carattere economico e politico».

4. Risposta di Benedetti Silvio, Parroco di San Baronto, lettera ms., S. Baronto, 2 novembre 1922, 1 c. Il Parroco di San Baronto risponde al Direttorio del fascio di Lamporecchio dicendosi sdegnato per le accuse infamanti ricevute sul suo conto. Egli si dice obbediente alle leggi e rispettoso delle autorità costituite.

5. Richiesta di iscrizione di Attilio Masi, carta ms., Lamporecchio, 8 luglio 1921, 1 c.

6. Richiesta di iscrizione di Giuseppe Pasquinucci, carta ms., Lamporecchio, 8 luglio 1921, 1 c.

2. Discorso contro il militarismo, minuta ms., s.l. [Lamporecchio], s.d. [1895-1915], $1 \mathrm{c}$.

3. Discorso sul Primo Maggio, minuta ms., s.l. [Lamporecchio], s.d. [1895-1915], 1 c.

4. Il Grande mutilato Carlo Del Croix, articolo di giornale, s.l., s.d. [1924], 1 c. Ritaglio di giornale con articolo dedicato al famoso mutilato di guerra, Carlo Delcroix, eletto deputato nella circoscrizione Toscana in occasione delle elezioni politiche del 1924.

5. Mentre d'autunno le brezze a gli alberi..., pergamena a stampa, s.l., s.d., 1 c. Poesia composta da sei ottavine e dedicata a due novelli sposi.

6. Tullio Bazzi, Giuseppe Giusti. Biografia aneddotica, opuscolo, Palermo, Casa Ed. Salvatore Biondo, s.d., 24 pp. Biografia del poeta e patriota Giuseppe Giusti (1809-1850).

7. Lettera anonima, carta stampata, s.l., s.d. [post 1905], 1 c. L'anonimo autore [probabilmente IT] denuncia «un individuo che non merita il nome d'uomo e che risponde a quello di Saul Massai oggi rifugiatosi a Lazzeretto, Comune di Cerreto Guidi». Tale Massai è accusato di essere un ladro, capace di «schifose e delittuose speculazioni, per cui gli fu possibile raggirare e turlupinare tanti galantuomini». Tra le vittime delle sue malefatte vi fu anche lo stesso IT. 
8. Articolo di giornale su comizio di IT, ritagli di giornale, s.l., s.d. [1922-23], 2 cc. Articolo intitolato Propaganda sindacale dedicato al comizio tenuto da IT a Fiesole sul tema 'Fascismo e Sindacalismo Nazionale'.

9. Alcuni intervenuti alla patriottica cerimonia, ritaglio di giornale, s.l., s.d., 1 c. Caricature di esponenti del fascismo della provincia di Firenze che hanno partecipato ad una cerimonia patriottica. Tra i raffigurati si riconosce anche IT, «Segretario Camera dei Sindacati Nazionali di Pontassieve».

10. Alfonso Merlini, La colonizzazione interna, opuscolo, Tip. Niccolai, Pistoia, 1901, 48 pp.

3. Scatola con cartoline, lettere e biglietti di condoglianze destinati alla famiglia Targioni per la morte di Idalberto. Contiene i seguenti elementi:

1. Cartolina di IT ai nipoti Rita, Lida e Idalberto Chiappini, cartolina ms., Lamporecchio, 21 dicembre 1921, 1 c. IT dedica dei versi ai suoi tre nipoti. Sul recto è presente un disegno raffigurante tre bambini che scendono le scale con vassoi ricchi di pietanze in mano.

2. Biglietti di condoglianze alla famiglia Targioni-Chiappini per la morte di Idalberto (25 maggio 1930):

1. Pietro Giannini e famiglia, biglietto a stampa e ms. + busta, Lamporecchio, 27 maggio 1930, 1 c.

2. Giannina Santirocchi, biglietto a stampa e ms. + busta, Lamporecchio, 28 maggio 1930, $1 \mathrm{c}$.

3. Risposta della famiglia Targioni-Chiappini a tale Virginia [Torrigiani], cameriera al servizio di Laura T. Merrick, biglietto a stampa e ms., Lamporecchio, 31 maggio 1930, 1 c.

4. Dott. Oliviero Pini, biglietto a stampa e ms. + busta, Lamporecchio, 31 maggio 1930, 1 c.

5. Eugenia Meozzi e famiglia, biglietto a stampa e ms. + busta, Lamporecchio, 27 maggio 1930, $1 \mathrm{c}$.

6. Bettina Pacchiani Verdiani e famiglia, biglietto a stampa e ms. + busta, Lamporecchio, 30 maggio 1930, 1 c.

7. Alfredo Venturini e famiglia, biglietto a stampa e ms. + busta, Lamporecchio, 27 maggio 1930, 1 c.

8. Cav. Oreste Talini e famiglia, biglietto a stampa e ms. + busta, Lamporecchio, 28 maggio 1930, $1 \mathrm{c}$.

9. Mancini Ilio e famiglia, biglietto ms. + busta, Lamporecchio, 28 maggio 1930, $1 \mathrm{c}$.

10. Allori Romualdo e famiglia, biglietto a stampa e ms. + busta, Lamporecchio, 30 maggio 1930, $1 \mathrm{c}$.

11. Luigi [Morelli], biglietto ms. + busta, Colle [?], 29 maggio 1930, $1 \mathrm{c}$.

12. Donati don Orlando Parroco di Porciano, biglietto a stampa e ms. + busta, Porciano, 28 maggio 1930, 1 c. 
13. Silvestri Pasquale, biglietto ms. + busta, Lamporecchio, 27 maggio 1930, $1 \mathrm{c}$.

14. Giuseppe e Giovanna Zucchi, biglietto a stampa e ms. + busta, Lamporecchio, 29 maggio 1930, 1 c.

15. Augusto Meozzi - Calzoleria, biglietto a stampa e ms., Lamporecchio, 27 maggio 1930, $1 \mathrm{c}$.

16. Famiglia Bertuccelli, biglietto ms. + busta, Lamporecchio, 31 maggio 1930, $1 \mathrm{c}$.

17. Dina Chiappini a Velia, lettera ms. + busta, Porciano, 27 maggio 1930, 2 cc. La Signora Dina Chiappini consiglia a Velia di portare presso di lei le sue bambine, «così si distrag[g]ono un po'».

18. Tito Borgioni - farmacista, biglietto a stampa e ms. + busta, Lamporecchio, 2 giugno 1930, $1 \mathrm{c}$.

19. Giannoni Ugo ad Amelia, lettera ms. + busta, S. Baronto, 1 giugno 1930, 2 cc.

20. Barbara Gavazzi ad Amelia, biglietto ms., Casamarconi, 2 giugno 1930, 2 cc.

21. Bacarelli Rizieri ad Amelia, lettera ms., Firenze, 28 maggio 1930, 2 cc.

22. Famiglia Pancani, biglietto a stampa e ms. + busta, Lamporecchio, 30 maggio 1930, $1 \mathrm{c}$.

23. Comm. Dott. Fazio Monzani, biglietto a stampa e ms. + busta, Firenze, 28 maggio 1930, $1 \mathrm{c}$.

24. Virginia Torrigiani a Velia, lettera ms. + busta, Villa Frassineto, 29 maggio 1930, 1 c.

25. Suore di Spicchio a Velia, biglietto ms. + busta, Lamporecchio, 30 maggio $1930,1 \mathrm{c}$.

26. Meozzi Luigi e Famiglia, biglietto a stampa e ms. + busta, Lamporecchio, 28 maggio $1930,1 \mathrm{c}$.

27. Iola e Berto Morelli, biglietto a stampa e ms. + busta, Lamporecchio, 3 giugno 1930, $1 \mathrm{c}$.

28. Omero Toti, biglietto a stampa e ms. + busta, Lamporecchio, 28 maggio 1930, $1 \mathrm{c}$.

29. Famiglia Morosi, biglietto a stampa e ms. + busta, Lamporecchio, 30 maggio $1930,1 \mathrm{c}$.

30. [Grafia non riconoscibile] ad Amelia, biglietto ms. + busta, Lamporecchio, 26 maggio 1930, 1 c.

31. Tranquillo Gasperini e famiglia, biglietto ms. + busta, Lamporecchio, 27 maggio 1930, $1 \mathrm{c}$.

32. Pancani Francesco - fabbrica di calzature, biglietto a stampa e ms. + busta, Lamporecchio, s.d., 1 c.

33. Alfieri e famiglia, biglietto ms. + busta, Lamporecchio, 29 maggio 1930, $1 \mathrm{c}$. 
34. Famiglia Matteucci, biglietto ms. + busta, Lamporecchio, 30 maggio 1930, $1 \mathrm{c}$.

35. Dott. Luigi Galeotti e famiglia, biglietto a stampa e ms. + busta, Lamporecchio, 28 maggio 1930, $1 \mathrm{c}$.

36. Famiglia Pancani, biglietto ms. + busta, Lamporecchio, 30 maggio 1930, $1 \mathrm{c}$.

37. Lidia e Alessandro Ubaldi, biglietto a stampa e ms. + busta, 28 maggio 1930, $1 \mathrm{c}$.

38. Innocenti Lindoro - Professore di Filosofia, biglietto a stampa e ms. + busta, 28 maggio 1930, $1 \mathrm{c}$.

39. Dott. Burdati, telegramma ms., Galluzzo, 28 maggio 1930, 1 c.

40. Martelli, telegramma ms., Roma, 28 maggio 1930, 1 c.

41. Capitano Tafini, telegramma ms., Lamporecchio, 28 maggio 1930, $1 \mathrm{c}$.

42. Fortunato Chiti, telegramma ms., Poggio a Caiano, 21 maggio 1930, 1 c. Chiti avverte IT che l'indomani sarebbe arrivato a fargli visita.

43. Filomena Chiti, telegramma ms., Poggio a Caiano, 8 maggio 1930, 1 c. Filomena Chiti avverte IT che «Fortunato trovasi a Genova».

44. Unione Provinciale Sindacati Agricoltura, telegramma ms., Firenze, 27 maggio 1930, $1 \mathrm{c}$.

45. Presidente Agricoltori Pondi, telegramma ms., Firenze, 26 maggio 1930, $1 \mathrm{c}$.

3. Richiesta offerta da parte dell'Istituto delle Suore terziarie Francescane di S. Giuseppe, lettera a stampa + busta, Quaracchi (Firenze), 3 giugno 1930, 1 c. La Suora Superiora del suddetto Istituto, Suor Giustina Stianti, chiede alla famiglia Targioni «un'offerta minima di L. 10, per una sola volta» per ricordare con preghiere quotidiane il defunto Idalberto davanti all'altare innalzato in onore del Patriarca S. Giuseppe.

4. Sollecito di versamento offerta dell'Istituto delle Suore terziarie Francescane di S. Giuseppe, lettera a stampa, Quaracchi (Firenze), s.d. [giugno 1930], 2 cc.

5. Federico Giraldi ad Amelia, lettera ms. + busta, Genova, 29 maggio 1930, 2 cc. Giraldi porge le più sentite condoglianze ad Amelia, esprimendo parole d'elogio per Idalberto «tanto buono, tanto di cuore, che seppe sempre con tutti far apprezzare le sue nobili doti».

6. Società Filarmonica "F. Berni" di Lamporecchio, lettera ms., Lamporecchio, 29 maggio 1930, 2 cc. Il Segretario della suddetta Filarmonica informa la famiglia Targioni di aver correttamente ricevuto la somma di denaro per aver prestato servizio al trasporto funebre di Idalberto e acclude le più sentite condoglianze. 
7. Fortunato Chiti ad Amelia, lettera ms. + busta, Poggio a Caiano, 28 maggio 1930, 1 c. Chiti porge le sue più sentite condoglianze ad Amelia e tesse le lodi del suo «carissimo amico Idalberto».

8. «Ricordo di Babbo. Contiene ricevuta del forno», busta da lettera ms., contiene i seguenti elementi:

1. Confraternita della Misericordia di Lamporecchio - Ricevuta del noleggio del carro funebre, carta ms., Lamporecchio, 28 maggio 1930, 2 cc.

2. Biglietto di condoglianze per la Famiglia Targioni-Chiappini, biglietto a stampa e ms., s.l., s.d., $1 \mathrm{c}$.

3. La morte di IT, ritaglio di giornale, s.l., s.d. [post 25 maggio 1930], 1 c. Ritaglio di giornale con articolo dedicato alla morte di IT, «Vice Segretario della Federazione Agricoltori di Firenze, [...] notissimo in tutta la Regione toscana per l'attività spiegata in favore delle organizzazioni sindacali».

9. A Rita Targioni, minute mss., Lamporecchio, 8 agosto 1905, 6 cc. Bozza di discorso pronunciato da IT sul feretro della figlia Rita, morta a nove anni l'8 agosto 1905.

10. Il mio testamento olografo, foglio a protocollo ms., Lamporecchio, 28 ottobre 1918, 2 cc. Bozza del testamento olografo di IT. In questa carta preparatoria di testamento (diverso rispetto a quello definitivo) parla solo della moglie Amelia come unica erede dei suoi beni (casa e mobilio della casa di Porciano e la somma di duemila lire).

11. IT a Velia, lettera ms., Firenze, 2 maggio 1925, 2 cc. IT informa Velia di non poter essere presente nel giorno della comunione della piccola Rita, figlia di Velia, e che avrebbe gradito essere avvertito in tempo per potervi prenderne parte. IT si abbandona poi a considerazioni malinconiche sulla vita e il pensiero corre subito verso un'altra Rita, sua figlia, deceduta nel 1905 .

12. IT a Velia, lettera ms., Firenze, 13 maggio 1925, 2 cc. IT informa Velia circa l'esito del processo conclusosi la mattina stessa.

13. IT a Velia, lettera ms., Firenze, 13 ottobre 1925, 2 cc. IT informa Velia circa il suo stato di salute in miglioramento e del suo arrivo a Lamporecchio previsto per il giorno 29 ottobre.

14. Carta datt. illeggibile, $1 \mathrm{c}$.

4. Carte sciolte - Articoli e corrispondenza su IT (post mortem).

1. Carlo Migliorati, Un poeta agitatore popolare. IT, ritaglio di giornale, «La Vita», Pistoia, 21 agosto 1966, 1 c. Articolo dedicato alla ricostruzione della biografia di IT attraverso i ricordi dell'autore. Si parla prevalentemente del contesto rurale in cui ha vissuto e agito IT e della sua poesia a favore dei contadini. Molte sono le informazioni imprecise fornite al lettore (luogo e data di nascita, cariche politiche mai assunte). 
2. Lida Chiappini al Direttore de «La Vita», lettera + busta, Lamporecchio, 23 agosto 1966, 1 c. Lida Chiappini rettifica i contenuti dell'articolo scritto da Carlo Migliorati.

3. Bruno Bruni a Lida Chiappini, lettera datt. + busta, Roma, 6 settembre 1966, 1 c. Bruni informa Lida di aver letto «la duplice nota su IT» sul settimanale pistoiese «La Vita» del 4 settembre, a cura di Carlo Migliorati, e di essere molto interessato alla produzione poetica di IT, «uno tra gli ultimi, in ordine di tempo, dei poeti popolari estemporanei».

4. Camera di Commercio di Pistoia al Dr. Bruno Bruni, lettera datt., Pistoia, 28 settembre 1967, 1 c. Si informa che l'articolo del Bruni sui poeti popolari pistoiesi è stato respinto perché la rivista «Pistoia» ha deciso di restituire carattere economico al periodico evitando «deviazioni storico-artistiche».

5. Bruno Bruni, lettera ms., s.l., 23 ottobre 1967, 1 c. L'autore esprime a qualcuno il disappunto per la mancata pubblicazione dell'articolo su «Pistoia».

6. Pier Paolo Borghi a Lida Chiappini, lettera ms. + foto-cartolina, Bologna, s.d. [1981], 3 cc. L'autore ringrazia Lida per avergli fornito preziose informazioni su IT, su cui, di lì a poco, avrebbe pubblicato un articolo su una rivista di tradizioni popolari di Reggio Emilia, «Il Cantastorie». Alla lettera, Borghi acclude una foto-cartolina raffigurante IT.

7. Chiappini Idalberto (nato l'8 giugno 1920, fratello di Lida e nipote di IT), Tema: Il mio paese, quaderno (fotocopia), Lamporecchio, s.d. [1928], $3 \mathrm{cc}$.

\section{Scatola 5}

\section{Consistenza: 263 op. a st., di cui 177 periodici; 5.475 pp.}

La scatola è prevalentemente composta da opuscoli e riviste, per la maggior parte dal contenuto politico, e da diverse copie di giornali, alcuni dei quali diretti da IT, altri contenenti suoi articoli e poesie inedite.

1. «Movimento operaio. Rivista di storia e bibliografia», libro rivista, a. 7, n.s. (mag.-ago. 1955) n. 3-4, Biblioteca Feltrinelli, Milano, 1955, 322 pp. Numerazione delle pagine parte dal n. 357. Alle pp. 511-530 cfr. Libertario Guerrini, Un poeta estemporaneo, IT, nella storia del movimento contadino dell'empolese.

2. Pensieri sulla questione sociale. Ricordo di una conferenza tenuta in Baragazza il di 24 agosto 1906 dal Prof. Luciano Milani Parroco di Settefonti, opuscolo, Bologna, Tip. Alfonso Garagnani, 1906, 32 pp.

3. B. Malon, La terza disfatta del proletariato francese, opuscoli a stampa, Firenze, Tip. Cooperativa, 1899, 132 pp. Diciassette dispense a stampa (n. 9, 12-16, 19-29) sulla storia della Comune di Parigi. 
4. «L'Autonomia Comunale. Bollettino dell'Associazione dei Comuni Italiani», Milano, 31 gennaio 1916, 16 pp.

5. «Francesco Ferrer», Firenze, 1 dicembre 1909, 8 pp. Giornale unico edito a cura dell'Associazione "Libero Pensiero".

6. «Lettura Socialista», rivista, Busseto (Parma), s.d. [pre 1915], 110 pp.

7. «Minerva», rivista, anno V, n. 12, s.l., dicembre 1895, 94 pp. La numerazione delle pagine parte dal n. 486.

8. Mario Cianchi, Giuseppe Garibaldi - Canto Popolare, opuscolo, Firenze, Tip. E. Ducci, s.d. [4 luglio 1907], 4 pp. Copertina di opuscolo, privo delle pagine interne e pubblicato, come si legge sul frontespizio, in « Ricordo del 1 Centenario della Nascita dell'Eroe», nato a Nizza il 4 luglio 1807.

9. «L'Università popolare - Rivista Quindicinale», rivista, anno II, n. 72, Mantova, Tip. Baraldi \& Fleishmann, 1 marzo1902, 32 pp.

10. Statuto della Società cooperativa di Consumo fra gli operai di S. Donato in Collina e di S. Cristofano situati nei Comuni di Bagno a Ripoli e di Rignano - Circondario di Firenze, opuscolo, Firenze, Tip. G. Civelli, 1897, 14 pp.

11. «Rivista di Milano. Politica e Lettere», rivista, anno II, n. 23, Milano, Stud. Edit. 'Corbaccio', 5 ottobre 1919, 66 pp.

12. «L'Etole Médicale. Rivista mensile di clinica medica e terapeutica», rivista, anno 17, n. 8, Milano-Parigi, Officine Grafiche D. Coen \& Co., 30 agosto 1910, $12 \mathrm{pp}$.

13. Virginia Paganini, Gli scogli che attraversano la Via Ascendente, opuscolo, Firenze, Tip. Cooperativa, 1904, 24 pp. Nell'ultima pagina presente bozza di componimento poetico.

14. «La Blouse. Rivista di letteratura operaia compilata esclusivamente con scritti di autentici lavoratori del braccio», rivista, anno III, n. 26, Firenze, maggio $1908,16 \mathrm{pp}$. La rivista presenta un grande strappo verticale che ha coinvolto tutte le pagine e la copertina.

15. Lo studio bolognese. Discorso di Giosuè Carducci per l'ottavo centenario, opuscolo, Bologna, Nicola Zanichelli, 1888, 46 pp.

16. Il Gruppo Parlamentare Socialista. L. Montemartini relatore, opuscolo, Roma, Tip. Popolare, 1908, 8 pp.

17. Relazione sulla Tattica e Programma per le prossime elezioni politiche e conseguente Azione Parlamentare. Costantino Lazzari relatore, opuscolo, Roma, Tip. Popolare, 1908, 12 pp.

18. «Il Socialismo», rivista, s.l., s.d., 12 pp. Rivista incompleta: presenti solo pp. 3-14.

19. Bacarelli Rizieri, Non di meno l'Autunno promette!, opuscolo, Firenze Tip. U. Polli, 1931, 28 pp. Raccolta di poesie. A p. 2, l'autore cita IT e Fortunato Chiti, dicendo al lettore di averli conosciuti. All'interno dell'opuscolo v'è una carta stampata, sciolta, intitolata Pensiero: La felici$t a \dot{~}$, firmato da Bacarelli Rizieri e datato 1932. 
20. F. Benuzzi, I versi d'un contadino, opuscolo, Lugano, Cooperativa Tipografica sociale, $1905,32 \mathrm{pp}$.

21. «Pagine Libere. Rivista Quindicinale di politica, Scienza ed Arte», rivista, anno III, n. 16, Lugano, S. A. Tipografica Luganese, 15 agosto 1909, 78 pp.

22. Epifane, Verso l'anarchia. Con lettera polemica di P. Kropotkine, opuscolo, Milano, Tip. Galimberti Politti \& Co., 1907, 60 pp.

23. Pompeo Ciotti, Sindacalismo Italico. Dalle origini all' insurrezione, opuscolo, Firenze, Casa Ed. Nerbini, 1906, 16 pp.

24. Socialismo e Antimilitarismo. Giovanni Bacci relatore, opuscolo, Roma, Tip. Popolare, 1908, 32 pp.

25. Pietro Gori, Il vostro Ordine e il nostro Disordine, opuscolo, Roma-Firenze, F. Sernatoni Ed., 1905, 16 pp.

26. «Battaglie d'oggi. Rivista di Socialismo Cristiano», rivista, anno VI, n. 7, Napoli, luglio 1910, 24 cc. + copertina, 48 pp.

27. A. Scalzotto, Tutti sindacalisti!, opuscolo, Mantova, Arturo Frizzi Ed., $1907,56 \mathrm{pp}$.

28. Dal Reno al Tevere. Scritto di grave interesse e opportunità per un cittadino romano, opuscolo, Napoli, Tip. A. Ferrante, 1870, 28 pp.

29. Filippo Turati, La vertigine degli armamenti e le riforme sociali. Discorso tenuto alla camera, 12 giugno 1909 (Resoconto stenografico), opuscolo, Mantova, Arturo Frizzi Editore, 1909, 32 pp.

30. Giuseppe Tesi, Religione e Cremazione, opuscolo, Pistoia, Tip. F.lli Ciattini, 1908, $32 \mathrm{pp}$.

31. Virginia Paganini, Lo scopo vero della nostra esistenza terrena, opuscolo, Firenze, Tip. Cooperativa, 1903, 48 pp., 2 copie.

32. Virginia Paganini, Il sentimento religioso - La Famiglia, opuscolo, Firenze, Tip. Cooperativa, 1888, 62 pp.

33. In umbra mortis. A ricordo di Gabbriella Camici e di Maria Camici-Fanoi, opuscolo, Pistoia, Tip. Niccolai, 2 novembre 1907, 24 pp.

34. F. Domela Niuwenhuis, Socialismo libertario e socialismo autoritario, opuscolo, Ancona, Tip. Economica Anconitana, 1896, 112 pp.

35. Demetrio Alati, Primo Maggio. Dramma in un atto, opuscolo, Firenze, G. Nerbini Editore, 1903, 40 pp.

36. A. Scalzotto, La tattica economica del partito socialista italiano. L'atteggiamento dei socialisti di fronte alle Società di Mutuo Soccorso, di Cooperazione e di Previdenza, Torino, Tip. Cesare Locatelli, 1897, 32 pp.

37. Il dovere e l'opportunità delle chiese evangeliche in rapporto colle questioni morali e sociali odierne, opuscolo, Prato, Tip. F.lli Passerini, 1906, 24 pp.

38. L'ultimo delitto dell'Inquisizione. Francesco Ferrer, opuscolo, Milano, Soc. Editrice 'La Milano', s.d. [1909], 32 pp.

39. Virginia Paganini, Saggio sulle dottrine di Giuseppe Mazzini - La nuova fede. Parole di Lina Cucco-Cacciamali, opuscolo, Firenze, Tip. Cooperativa, 1890, $32 \mathrm{pp}$. 
40. Zagaglia, I coattipolitici in Italia, opuscolo, Roma, Tip. Ed. Sociale, 1895, $48 \mathrm{pp}$.

41. Trittico della morte della Luna, porzione di opuscolo, s.l., s.d., 12 pp.

42. Camillo Ventura, La poesia e le leggi della natura, opuscolo, Milano, Ditta Natale Battezzati, 1888, 80 pp.

43. Scuola Professionale di Colle Val D'Elsa, Statuto, regolamento e programmi didattici della scuola, opuscolo, Siena, Tip. Sordo-Muti di L. Lazzeri, 1894, $48 \mathrm{pp}$.

44. Pietro Gori, Ricordi, opuscolo, Milano, Libreria Editrice Sociale, 1911, $48 \mathrm{pp}$.

45. Refezione scolastica nel Comune di Colle Val D'Elsa, Relazione del primo anno di esercizio 1901, Colle Val D'Elsa, Tip. Meoni, 1901, 16 pp.

46. Fratellanza di Mutua e Pubblica Assistenza in Campi Bisenzio, Charitas. Ricordo del XXV Anniversario di fondazione, opuscolo, Firenze, Tip. F.lli Fioretti, 1897, $46 \mathrm{pp}$.

47. Ottavio Dinale, Diversità di tendenze o Equivoco?, opuscolo, Firenze, G. Nerbini Editore, 1902, 16 pp.

48. Virginia Paganini, Guida Morale e Pratica per le madri del popolo, opuscolo, Firenze, Tip. Cooperativa, 1889, 100 pp.

49. Edgard Milhaud, Cooperazione e socialismo (Che cosa è avvenuto in Germania), opuscolo, Milano, Tip. Coop. Comense, marzo-aprile 1914, 96 pp.

50. «Il Socialismo. Rivista quindicinale diretta da Enrico Ferri», a. I, n. 11, Roma, 25 luglio 1902, 20 pp.

51. Incitamento agli italiani a rivolgere ormai tutte le loro facoltà morali e fisiche al solo nobile scopo dell'unità nazionale italiana d'un Vigile Romagnuolo, opuscolo, Italia, $1847,64 \mathrm{pp}$. La copertina presenta iscrizioni mss. ad opera di IT.

52. F. D. Guerrazzi, Leopoldo II, opuscolo, Tip. Torelli, Firenze, 1859, 12 pp. Sull'ultima pagina, iscrizione riconducibile alla grafia di IT.

53. La coltivazione della vite. Osservazioni ed esperienze d'un agricoltore, opuscolo, Tip. Giachetti, Prato, 1902, 16 pp.

54. Oddino Morgari, Nicola Secondo Czar di tutte le Russie ai Liberali d'Italia per l'arbitrato internazionale e per disarmo. Discorso tenuto alla Camera il 23 Giugno 1909 (Resoconto stenografico), opuscolo, Arturo Frizzi Editore, Mantova, 1909, 32 pp.

55. Società di Mutuo Soccorso tra gli Operai del Comune di Lamporecchio, Rapporto morale e amministrativo dei Sindaci sul consuntivo per la gestione 1891, opuscolo, Tip. Niccolai, Pistoia, 1892, 12 pp.

56. Relazione del Commissario Prefettizio Cav. Colombo Pellegrini al Consiglio Comunale di Lamporecchio nella seduta inaugurale del 6 novembre 1920, opuscolo, Ditta Alberto Pacinotti \& C., Pistoia, 1920, 32 pp. A p. 12 è presente un appunto ms. ad opera di IT 
57. Nuovo Regolamento Generale per l'Istruzione elementare. Emanato con Regio Decreto, Ottobre 1895, opuscolo, R. Bemporad e Figlio, Firenze, 1895, 60 pp.

58. Regolamento per l'esecuzione della legge 31 maggio 1903, n. 254 sulle Case Popolari approvato con Regio Decreto 24 aprile 1904, n. 164, opuscolo, Casa Editrice E. Pietracola, Napoli, 1906, 48 pp.

59. Leggi, Decreti e Regolamenti per l'applicazione della Imposta sui Fabbricati, opuscolo, Casa Editrice E. Pietracola, Napoli, 1908, 116 pp.

60. Errico Malatesta, L'Anarchia, opuscolo, Casa Editrice Libraria 'Il Pensiero', Roma, 1907, 64 pp.

61. E.C. Longobardi, Direttiva del Partito in rapporto al movimento operaio, opuscolo, Tip. Popolare, Roma, 1908, 24 pp.

62. C. Monticelli, Lo sciopero. Storia suggestiva di uno sciopero, opuscolo, Libreria Editrice 'Luigi Mongini', Roma, 1910, 8 pp.

63. Alceste Della Sete, Il Partito Socialista e le questioni dell'antimilitarismo. Relazione sul tema delle spese militari ed antimilitarismo, opuscolo, Coop. Tipografica 'Avanti', Roma, 1910, 32 pp.

64. Le condizioni del clero. Lettera aperta a S. E. il Cardinale Prisco, Arcivescovo di Napoli, in occasione della condanna del periodico «Battaglie d'Oggi», opuscolo, Tip. Alfredo De Frede, Napoli, 1909, 20 pp.

65. Giuseppe Mazzini, Missione italiana, vita internazionale. Nazionalismo e nazionalità, opuscolo, Casa Editrice Nerbini, Firenze 1916, 16 pp. Presenti iscrizioni mss. sul retro della copertina, probabilmente ad opera di IT.

66. Giorgio Renard agli studenti, opuscolo, Ufficio della Lotta di Classe, Milano, $1895,16 \mathrm{pp}$. Sull'ultima pagina è presente un'iscrizione ms. probabilmente realizzata da IT.

67. Pompeo Ciotti, Gli agguati alla consorteria. Maggio 1898 - Cinque anni dopo, opuscolo, G. Nerbini Editore, Firenze, 1903, 32 pp.

68. Pietro Lapucci, Compendio di Storia e Geografia della Provincia di Livorno, opuscolo, Raffaello Giusti Libraio-Editore, Livorno, 1887, 48 pp.

69. Giulio de' Rossi, Il Primo Maggio. Alla Società di Mutuo Soccorso fra gli Operai di Pistoia nel trentesimo anniversario della sua fondazione, Tip. Niccolai, Pistoia, 1891, 62 pp.

70. Sul bilancio dell'Interno. Discorsi dei deputati Gatti-Badaloni - Ferri pronunziati alla Camera dei Deputati nelle tornate del 17, 18 e 22 giugno 1901, Tip. della Camera dei Deputati, Roma, 1901, 140 pp. A p. 2 è presente un elenco di parole probabilmente scritte da IT come bozza per la composizione di rime alternate.

71. Per le terre dell' immenso dolore in «Lettura Socialista», s.a., s.n., Busseto (Parma), 1909, 96 pp.

72. Ferdinando Martini, Per la Guerra. Discorso pronunziato a Firenze nel Salone dei Cinquecento da Ferdinando Martini, Ministro delle Colonie, il XX gennaio MCMXVI, opuscolo, Unione Editrice, Roma, 1916, 20 pp. 
73. Giuseppe Manzini, Libero Amore (Studio di Sociologia Moderna), libro a stampa, Casa Tipo-Lito Edit. Sinibuldiana G. Flori e C., Pistoia, 1905, 96 pp.

74. Federico De Maria, Poema Libero, libro a stampa, Edizioni di 'Poesia', Milano, 1909, 272 pp.

75. Omero, L'Iliade. Traduzione di Ettore Romagnoli con introduzione e commento di G. B. Salinari, parte di copertina a stampa, Zanichelli, Bologna, s.a., 2 pp.

76. Statuto della Società di Mutuo Soccorso fra gli operai del Comune di Lamporecchio, opuscolo, Tip. Niccolai, Pistoia, 1906, 36 pp.

77. Parole dette nella Chiesa di Lamporecchio il di 9 luglio 1894 alla mia nepote Giulia Galeotti ed al suo sposo Dottor Guido Del Vivo, parte di opuscolo, s.l., s.d., 34 pp. (presenti solo pp. 97-130).

78. Il Partito Socialista Italiano e la politica dell'emigrazione. Relatore Angiolo Cabrini, opuscolo, Tip. Popolare, Roma, 1908, 24 pp.

79. Giuseppina Martinuzzi, Nazionalismo morboso e Internazionalismo affarista, Tip. Brunner \& Co., Trieste, 1911, 40 pp.

80. Discorso del deputato Casciani (Relatore del Bilancio) pronunziati alla Camera dei Deputati nelle tornate del 23, 24, 25 e 26 marzo 1904, Tip. Camera dei Deputati, Roma, 1904, 60 pp.

81. Francesco Di Marco Datini, Mercante e Benefattore. Discorso letto da Isidoro del Lungo il di 18 ottobre 1896 nell' inaugurazione della statua in Prato, Tip. Giachetti, Prato, 1897, 30 pp.

82. Mario Rapisardi, Il Prometeo liberato, Casa Editrice Nerbini, Firenze, 1908, 16 pp.

83. Emidio Cesari, Cos'è e come funzionerà in Italia lo scrutinio di lista con la rappresentanza proporzionale, Caesar, Ascoli Piceno, $32 \mathrm{pp}$.

84. Giovanni Pastorello, Lo Stato Collettivista, Libreria Editrice Piccarolo, Torino, 1900, $96 \mathrm{pp}$.

85. Il Comune fiorentino e la scuola laica, Resoconto stenografico della Seduta Consiliare del 16 settembre 1907, Casa Editrice Nerbini, Firenze, 1907, 44 pp.

86. Edmondo De Amicis, Consigli e moniti, Libreria Editrice Nerbini, Firenze, s.d., 14 pp.

87. Giovanni Martini, Vade Mecum del Contadino Toscano, Tip. Ciattini, Pistoia, 1907, $48 \mathrm{pp}$.

88. Antonio Benesperi, La coltura della vite. Sistema del colono Vincenzo Guazzini ampliato e svolto, Tip. Giachetti, Prato, 1903, 86 pp.

89. F. Felice da Porretta, La cremazione dei cadaveri, Tip. Sinibuldiana, 1907, $32 \mathrm{pp}$.

90. «Luce e ombra», rivista, anno VIII, fascicolo 5, Milano, maggio 1908, 54 pp. (pp. 221-276).

91. «La Nuova Parola. Rivista illustrata d'attualità dedicata ai nuovi ideali nell'Arte della Scienza nella Vita», rivista, anno II, n. 4-5, vol. III, Roma, 1903, 110 pp. (pp. 241-350). 
92. Per un episodio della questione parrocchiale in Montevettolini, opuscolo, Montevettolini, giugno 1914, $16 \mathrm{pp}$.

93. A. Angiolini, Socialismo e socialisti in Italia, parte di opuscolo, Firenze, Tip. Cooperativa, 1899, 8 pp.

94. «La Martinella. Periodico settimanale. Organo socialista toscano», anno XX, n. 24, Colle Val d'Elsa, 15 giugno 1901, 4 pp.

95. «La Martinella», anno XX, n. 22, Colle Val d'Elsa, 1 giugno 1901, 4 pp.

96. «La Martinella», anno XX, n. 9, Colle Val d'Elsa, 2 marzo 1901, 4 pp.

97. «La Bandiera del Popolo. Settimanale del Circondario di Pistoia», anno III, n. 15, Pistoia, 16 aprile 1922, 6 pp.

98. «La Bandiera del Popolo», anno III, n. 16, Pistoia, 23 aprile 1922, 6 pp.

99. «La Bandiera del Popolo», anno III, n. 19, Pistoia, 14 maggio 1922, 6 pp.

100. «La Bandiera del Popolo», anno III, n. 21, Pistoia, 28 maggio 1922, 6 pp.

101. «La Bandiera del Popolo», anno III, n. 24, Pistoia, 18 giugno 1922, 6 pp. 102. «La Bandiera del Popolo», a. V, n. 37, Pistoia, 12 ottobre 1924, 4 pp. A p. 3 , pubblicata lettera rivolta al Direttore del giornale, nel quale si comunica che IT ha sporto querela nei confronti della testata e di un giornalista reo di averlo «ingiustamente ingiuriato e diffamato». Nella stessa pagina IT viene menzionato nell'articolo Corrispondenze da Lamporecchio. Diciotto mesi di amministrazione fascista.

103. «Il Rinnovamento della scuola», Roma, 13 maggio 1961, 4 pp. (di proprietà di Lida Chiappini).

104. «L'Appennino Toscano», Borgo S. Lorenzo, 10 ottobre 1926, 4 pp.

105. «L'Asino. E il popolo utile paziente e bastonato», a. XII, n. 17, Roma, 1 maggio 1903, 8 pp.

106. «L'Asino», a. XII, n. 41, Roma, 8 novembre 1903, 8 pp.

107. «L'Asino», a. XII, n. 45, Roma, 15 novembre 1903, 8 pp.

108. «L'Asino», a. XIV, n. 49, Roma, 3 dicembre 1905, 8 pp.

109. «L'Asino», a. XVII, n. 12, Roma, 22 marzo 1908, 8 pp.

110. «L'Asino», a. XVII, n. 33, Roma, 16 agosto 1908, 8 pp.

111. «L'Asino», anno XVIII, n. 48, Roma, 25 ottobre 1908, 8 pp.

112. «L'Asino», a. XVII, n. 44, Roma, 1 novembre 1908, 8 pp.

113. «L'Asino», a. XVIII, n. 14, Roma, 4 aprile 1909, 4 pp.

114. «L'Asino», a. XVIII, n. 25, Roma, 20 giugno 1909, 8 pp.

115. «L'Asino», a. XVIII, n. 42, Roma, 17 ottobre 1909, 8 pp.

116. «L'Asino», a. XVIII, n. 47, Roma, 21 novembre 1909, 8 pp.

117. «L'Asino», a. XVIII, n. 48, Roma, 28 novembre 1909, 8 pp.

118. «L'Asino», a. XIX, n. 3, Roma, 16 gennaio 1910, 8 pp.

119. «L'Asino», a. XIX, n. [?], Roma, [?] 1910, 4 pp. (pagine mancanti)

120. «L'Asino», anno XIX, n. 6, Roma, 6 febbraio 1910, 4 pp.

121. Miracoli e delitti degli impostori, ritaglio di giornale, s.l., s.d., 1 c. 
122. «Il Risveglio del Mont'Albano», a. I, n. 1, febbraio 1908, 4 pp. Articoli di IT a p. 1, La mesata politica. Su Lamporecchio cfr. pp. 1-2, Cose comunali. Novità, e p. 3, Cristo in giro per Lamporecchio.

123. «Il Risveglio del Mont'Albano», a. I, n. 5, giugno 1908, 4 pp. Articolo di IT a p. 1, La neutralità del governo, e a p. 3, Corrado Giunti; poesia a p. 2, Favola sociale.

124. «Il Risveglio della plebe», a. I, n. 7, 23 luglio 1908, 4 pp. Articoli di IT a p. 1, Francesco Minghetti [in memoria di], e a p. 3, Combattiamo il prete.

125. «Il Risveglio della plebe», a. I, n. 9, 18 settembre 1908, 4 pp. Articolo di IT a p. 3, $A^{\prime}$ miei critici.

126. «Il Risveglio della Plebe», anno I, n. 11, S. Baronto, 5 novembre 1908, 4 pp. Articoli di IT a p. 1, La città deimorti, e p. 2, con una poesia intitolata Il Ragno.

127. «Il Risveglio della plebe», a. I, n. 20, 3 dicembre 1908, 4 pp. Articolo di IT a p. 1, Rod-Bod, e poesia a p. 2, L'Inverno e le sue conseguenze.

128. «Il Risveglio della plebe», Lamporecchio, 28 maggio 1909, 4 pp.

129. «Il Risveglio della plebe», a. II, n. 34, 25 giugno 1909, 4 pp. Articoli di IT a p. 1, La Chiesa e la Storia, e p. 3, Ignoranti e superbi!.

130. «Il Risveglio della plebe», a. II, n. 39, 5 novembre 1909, 4 pp. Articoli di IT a p. 1, Francisco Ferrer assassinato per opera del Gesuitismo e del Vaticano, e a p. 3, Lo Czar non è venuto in Italia.

131. «Il Risveglio della plebe», a. II, n. 7, 23 luglio 1908, 4 pp.

132. «Il Risveglio della plebe», a. II, n. 37, S. Baronto, 3 settembre 1909, 4 pp. Articolo di IT alle pp. 1-2, Piccoli contribuenti e nullatenenti.

133. «Il Risveglio della plebe», a. III, n. 1, 14 gennaio 1910, 4 pp.

134. «Il Risveglio della plebe», a. III, n. 2, 21 gennaio 1910, 4 pp. Articoli di IT a p. 1, Macchiavellismo borghese, alle pp. 1-2, Il Dio Progresso e le sue vittime, e a p. 2, Un ordine del giorno della Sezione socialista di Lamporecchio.

135. «Il Risveglio della plebe», a. III, n. 3, 28 gennaio 1910, 4 pp., 2 copie. Articolo di IT a p. 1, Per la morte di Andrea Costa. É passato. Su Lamporecchio cfr. p. 3, Corriere di Lamporecchio. Un'altra del Sindaco.

136. «Il Risveglio della plebe», a. III, n. 7, 25 febbraio 1910, 4 pp.

137. «Il Risveglio della plebe», a. III, n. 25, 24 giugno 1910, 4 pp.

138. «Avanti! Della Domenica», a. II, n. 48, Roma, 28 luglio 1904, 4 pp.

139. «L'Avvenire. Organo Socialista settimanale del Circondario di Pistoia», Pistoia, a. III, n. 32, 2 agosto 1903, 4 pp. Articolo di IT alle pp. 1-2, Alla cittadinanza pistoiese dopo la lettera dell'avv. Alfredo Pasquali alla Camera del Lavoro.

140. «L'Avvenire», Pistoia, a. IV, n. 6, 7 febbraio 1904, 4 pp. Articolo di IT alle pp. 1-2, Dai Santi Padri a Leone XIII. Dedicato agli Scagnozzi della DIFESA.

141. «L'Avvenire», Pistoia, a. IV, n. 13, 27 marzo 1904, 4 pp. Articolo di IT a p. 1, Per le due tendenze.

142. «L'Avvenire», Pistoia, a. IV, n. 14, 3 aprile 1904, 4 pp. 
143. «L'Avvenire», Pistoia, a. IV, n. 27, 3 luglio 1904, 4 pp. Articolo di IT a p. 1, I veri miserabili.

144. «L'Avvenire», Pistoia, a. IV, n. 30, 24 luglio 1904, 4 pp.

145. «L'Avvenire», Pistoia, a. V, n. 38, 17 settembre 1905, 4 pp. Poesia di IT a p. 2, Il terremoto.

146. «L'Avvenire», Pistoia, a. VII, n. 34, 25 agosto 1907, 4 pp.

147. «L'Avvenire», Pistoia, a. VIII, n. 21, 24 maggio 1908, 4 pp. Articolo di IT a p. 1, La borghesia capitalista nella Svizzera.

148. «L'Avvenire», Pistoia, a. VIII, n. 36, 25 luglio 1908, 4 pp. Articolo di IT alle pp. 1-2, A Don Alighiero Ciattini Vescovo dei sindacalisti Pistoiesi.

149. «L'Avvenire», Pistoia, a. VIII, n. 41, 4 ottobre 1908, 4 pp. Articolo di IT alle pp. 1-2, L'ora presente.

150. «L'Avvenire», Pistoia, a. VIII, n. 52, 25 dicembre 1908, 4 pp. Poesia di IT a p. 2, Cose che succedono ovvero Le avventure d'un mendicante e d'un ruffiano galoppino, monarchico, clericale.

151. «L'Avvenire», Pistoia, a. IX, n. 1, 3 gennaio 1909, 4 pp. Articolo di IT a p. $1,1909$.

152. «L'Avvenire», Pistoia, a. IX, n. 15, 11 aprile 1909, 4 pp. Articolo di IT a p. 1, Pasqua.

153. «L'Avvenire», Pistoia, a. IX, n. 42, 24 ottobre 1909, 4 pp. Articolo di IT a p. $1, \grave{E}$ bene che venga!

154. «L'Avvenire», Pistoia, a. IX, n. 43, 31 ottobre 1909, 4 pp. Articoli di IT a p. 1, Lo Czar non è venuto in Italia, alle pp. 2-3, Ranocchie clericali, e a p. 3, Dalle stinche.

155. «L'Avvenire», Pistoia, a. IX, n. 51, 19 dicembre 1909, 4 pp. Articolo di IT a p. 1, Il caso Ferri.

156. «L'Avvenire», Pistoia, a. X, n. 1, 1 gennaio 1910, 4 pp., 2 copie. Articolo di IT alle pp. 1-2, Lettera aperta al signor Milziade Ricci Direttore del "Pistoia Nuova".

157. «L'Avvenire», Pistoia, a. X, n. 9, 27 febbraio 1910, 4 pp. Articolo di IT alle pp. 1-2, La difesa di un vinto.

158. «L'Avvenire», Pistoia, a. X, n. 15, 10 aprile 1910, 4 pp. Articolo di IT a p. 1, L'Incognita" del Suffragio Universale.

159. «L'Avvenire», Pistoia, a. X, n. 28, 10 luglio 1910, 4 pp. Articolo di IT a p. 1-2, "Non fui lenone".

160. «L'Avvenire», Pistoia, a. X, n. 32, 7 agosto 1910, 4 pp. Articolo su IT a p. 1, Vertenza Medri-Targioni-Berni.

161. «L'Avvenire», Pistoia, a. XI, n. 12, 19 marzo 1911, 4 pp. Articoli su IT a p. 1, La nostra condanna e In Corte d'Assise.

162. «L'Avvenire», Pistoia, a. XI, n. 13, 26 marzo 1911, 4 pp. Articolo su IT a p. 2, Per un comizio alle Piastre.

163. «L'Avvenire», Pistoia, a. XI, n. 20, 14 maggio 1911, 4 pp. Articolo di IT a p. 1, L'intelligenza dei brigadieri. 
164. «L'Avvenire», Pistoia, a. XI, n. 27, 2 luglio 1911, 4 pp. Articolo di IT, Ai liberipensatori.

165. «L'Avvenire», Pistoia, a. XI, n. 40, 1 Ottobre 1911, 4 pp.

166. «L'Avvenire», Pistoia, a. XI, n. 47, 19 novembre 1911, 4 pp.

167. «L'Avvenire», Pistoia, a. XI, n. 50, 10 dicembre 1911, 4 pp. Articolo di IT alle pp. 1-2, Lettera aperta all'on. Giovanni Giolitti Ministro degl'Int.

168. «L'Avvenire», Pistoia, a. XI, n. 51, 17 dicembre 1911, 4 pp. Articolo su IT a p. 2, Contraddittorio Giannoni-Targioni.

169. «L'Avvenire», Pistoia, a. XII, n. 2, 11 gennaio 1912, 4 pp.

170. «L'Avvenire», Pistoia, a. XII, n. 19, 12 maggio 1912, 4 pp. Articolo su IT a p. 3, Corriere di Lamporecchio. Gl'incontentabili.

171. «L'Avvenire», Pistoia, a. XIII, n. 9, 2 marzo 1913, 4 pp.

172. «L'Avvenire», Pistoia, a. XIII, n. 12, 23 marzo 1913, 4 pp. Articolo di IT a p. 1, Intervista coll'on. Valentino Pittoni Deputato al Parlamento Austriaco sulle Cooperative di Consumo.

173. «L'Avvenire», Pistoia, a. XIII, n. 45, 9 novembre 1913, 4 pp.

174. «L'Avvenire», Pistoia, a. XIV, n. 29, 19 luglio 1914, 4 pp. A p. 1-2, articolo su La clamorosa vittoria socialista di Lamporecchio, che decretò l'elezione a sindaco di IT.

175. «L'Avvenire», Pistoia, a. XIV, n. 31, 2 agosto 1914, 4 pp. Articolo su IT a p. 3, Corrispondenze da Lamporecchio.

176. «L'Avvenire», Pistoia, a. XIV, n. 43, 25 ottobre 1914, 4 pp. Articolo su IT a p. 3, Da Lamporecchio.

177. «L'Avvenire», Pistoia, a. XIV, n. 44, 1 novembre 1914, 4 pp. Articolo su IT a p. 3, Da Lamporecchio.

178. «L'Avvenire», Pistoia, a. XI, n. 7, 14 febbraio 1914, 4 pp. Articolo di IT alle pp. 2-3, Ultima e definitiva risposta all'anonimo del "Popolo Pistoiese". Da Lamporecchio.

179. Bollettino elettorale. Lettera-Programma dei Partiti Popolari di Lamporecchio, manifesto a stampa, Lamporecchio, 27 luglio 1910, 2 pp. A p. 2, discorso di IT rivolto ai cittadini-elettori, Ai cittadini liberi di Lamporecchio.

180. Jessie W. Mario, Della vita di Giuseppe Mazzini, opuscoli a stampa, Milano, Tip. della Società Editrice Sonzogno, 1885,100 pp. Vita di Giuseppe Mazzini raccontata a puntate con tanto di illustrazioni. Raccolta incompleta, presenti solo 13 dispense su un totale di 62.

181. «L'Alleanza. Periodico quindicinale antibolscevico di critica e di controllo per la difesa sociale e gli interessi agricoli e commerciali dei Comuni limitrofi di Montalbano», a. I, n. 1, Lamporecchio, 1 gennaio 1921, 4 pp. Articolo di IT a p. 3, Moniti e consigli agli operai e in difesa del socialismo - prima parte.

182. «L'Alleanza», a. I, n. 2, Lamporecchio, 15 gennaio 1921, 4 pp., 2 copie. Articolo di IT a p. 1, Moniti e consigli agli operai e in difesa del socialismo - seconda parte e a p. 3, Lettera aperta al sindaco di Lamporecchio. 
183. «L'Alleanza», a. I, n. 3, Lamporecchio, 31 gennaio 1921, 4 pp. Articoli di IT alle pp. 1-2, Nella Chiesa Rossa - Dopo il Congresso di Livorno, e a p. 3, Il nostro socialismo.

184. «L'Alleanza», a. I, n. 5, Lamporecchio, 4 marzo 1921, 4 pp. Articoli di IT a p. 1, Democrazia rurale. Ritorniamo alla Terra - prima parte, alle pp. 1-2, La parola all'imputato, e alle pp. 3-4, $2^{\circ}$ lezione di educazione morale e di coraggio civile al Sindaco di Lamporecchio.

185. «L'Alleanza», a. I, n. 6, Lamporecchio, 17 marzo 1921, 4 pp. Articoli di IT a p. 2, Democrazia rurale. Ritorniamo alla Terra - seconda parte, e alle pp. 2-3, La parola all'imputato - seconda parte.

186. «L'Alleanza», a. I, n. 7, Lamporecchio, 31 marzo 1921, 4 pp. Articoli di IT a p. $3,3^{\circ}$ lezione al Sindaco di Lamporecchio data per gli stessi titoli e motivi della seconda, e p. 4, Finalmente rispondono.

187. «L'Alleanza», Lamporecchio, 21 aprile 1921, 4 pp. Articoli di IT alle pp. 1-2, Agli operai delle fabbriche e dei campi. In difesa del fascismo calunniato, e a p. 2, Operai Contadini e Braccianti. Per chi dovete votare?. A p. 4 si dà notizia della prossima inaugurazione del Fascio di combattimento di Lamporecchio: fra gli oratori era previsto anche l'intervento di IT.

188. «L'Alleanza», a. I, n. 11, Lamporecchio, 6 maggio 1921, 4 pp., 3 copie. Articolo di IT a p. 3, Maggio 1921 (dedicato ai contadini). A p. 4, in Avviso Importante - Da Larciano, si dà notizia di un prossimo comizio elettorale a Larciano con oratori l'on. Dino Philipson e Carlo Martelli, candidati per il blocco, e di IT.

189. «L'Alleanza», a. I, n. 12, Lamporecchio, 13 maggio 1921, 4 pp. Articolo di IT a p. 1, Battaglia campale - Tutti alle urne! Per la nuova e grande Italia, e poesia dello stesso autore a p. 2, 5 maggio 1921. Canto nuovo all'Italia.

190. «L'Alleanza», a. I, n. 13, Lamporecchio, 27 maggio 1921, 4 pp., 4 copie. Articolo di IT a p. 1, Dopo la battaglia. Tutti sconfitti - Un solo Vincitore - Il Fascismo.

191. «L'Alleanza», a. I, n. 14, Lamporecchio, 11 giugno 1921, 4 pp. Articolo di IT a p. 1, Per dissipare gli equivoci nel campo nostro e in quello degli altri. Il Fascismo.

192. «L'Alleanza», a. I, n. 16, Lamporecchio, 14 luglio 1921, 4 pp. Articolo di IT a p. 1, Per la pace tra fascisti e socialisti, alle pp. 1-2, Risposta al gruppetto de popolari ed ai parroci del Comune di Lamporecchio, a p. 2, Alla "Bandiera del Popolo" ed ai corrispondenti di Orbignano e Porciano, e a p. 3, Seconda lettera aperta al sig. Avv. Pietro Bianchi Segretario Comunale di Lamporecchio.

193. «L'Alleanza», a. I, n. 17, Lamporecchio, 28 luglio 1921, 4 pp., 3 copie. Articoli di IT a p. 1, La Toscana in framme, e alle pp. 2-3 (Seconda e ultima parte della seconda risposta alla seconda lettera aperta dell'avv. Pietro Bianchi. A p. 4, Fasci Italia di Combattimento Sezione di Lamporecchio. Per l'inaugurazione del gagliardetto. 
194. «L'Alleanza», a. I, n. 18, Lamporecchio, 11 agosto 1921, 4 pp. Articoli di IT a p. 2, Il giuri d'onore, p. 3, Luce, luce, luce, ma luce su tutto; Documenti, e a p. 4, Risposta alla lettera aperta di Don Silvio Benedetti parroco di S. Baronto.

195. «L'Alleanza», a. I, n. 19, Lamporecchio, 1 settembre 1921, 4 pp. 3 copie. Articoli di IT a p. 1, Le vie nuove del fascismo, p. 2, Osservazioni intorno al trattato di pace, e a p. 3, Giuri d'onore che sfuma.

196. «L'Alleanza», a. I, n. 20, Lamporecchio, 16 settembre 1921, 4 pp. Articoli di IT a p. 1, Crisi su tutta la linea, e a p. 3, Tutto il mondo festeggia il poeta e Ancora del semolino e del riso. Serie di articoli sulla polemica ITBianchi in merito alla cattiva gestione dello Spaccio Comunale.

197. «L'Alleanza», a. I, n. 21, Lamporecchio, 29 settembre 1921, 4 pp. Articoli di IT a p. 1,E muta nome perché muta lato e Per la repressione della guerriglia civile, e a p. 3, La fine d'una polemica. Ancora articoli sulla polemica IT- Bianchi in merito alla cattiva gestione dello Spaccio Comunale.

198. «L'Alleanza», a. I, n. 22, Lamporecchio, 27 ottobre 1921, 4 pp., 2 copie. A p. 1, poesie dedicatorie di IT ai defunti Gino Pacini, Segretario del Fascio di Cerreto Guidi, e Italo Gambacciani del Fascio di Montelupo. Articoli di IT a p. 2, Congedo (in cui si annuncia la fine della pubblicazione de «L'Alleanza» e l'inizio della messa in stampa de «L'Ordine») e a p. 3, L'Italia in questo momento.

199. «L'Ordine. Organo settimanale di critica e di controllo per la difesa degli interessi agricoli, industriali e commerciali del Circondario Pistoiese», a. II, n. 1, Pistoia, 1 gennaio 1922, 4 pp., 3 copie. Articolo di IT a p. 1, Capo d'anno 1 gennaio 1922. Articolo su Lamporecchio a p. 4, Corrispondenza dai Comuni del circondario. Da Lamporecchio.

200. «L'Ordine», a. II, n. 2, Pistoia, 8 gennaio 1922, 4 pp., 3 copie. Articoli di IT a p. 1, Ricostruzione economica e disarmo, e a p. 2, Sebastiano Del Bono. 201. «L'Ordine», a. II, n. 3, Pistoia, 15 gennaio 1922, 4 pp., 3 copie.

202. «L'Ordine», a. II, n. 4, Pistoia, 22 gennaio 1922, 4 pp. Articoli di IT a p. 1, Una sola via rimane aperta all'Italia. O produr di piu di quanto si produce o perire, alle pp. 3-4, Dai Comuni del Circondario. Da Lamporecchio. Carlo Minghetti [in memoria di] e a p. 4, Fascio Italiano di Combattimento di Lamporecchio e Da Tizzana. La canaglia popolar-pussista contro IT sabato sera 14 corr. alla Catena.

203. «L'Ordine», a. II, n. 5, Pistoia, 29 gennaio 1922, 4 pp., 3 copie. Articoli di IT alle pp. 1-2, Il gigante disoccupato, a p. 3, Il Montalbano. Articolo su Lamporecchio a p. 4, Dai Comuni del Circondario. Da Lamporecchio.

204. «L'Ordine», a. II, n. 8, Pistoia, 19 febbraio 1922, 4 pp., 2 copie. Articolo di IT alle pp. 1-2, Come si coltivano fallaci speranze e si propaga l'odio di classe. Notare a p. 3, Dai Comuni del Circondario. Da Lamporecchio, in cui si fa riferimento alla negligente condotta di Torello Chiappini, genero di IT, alla guida dello Spaccio Comunale. 
205. «L'Ordine», a. II, n. 10, Pistoia, 5 marzo 1922, 4 pp. Articoli di IT a p. 1, Il Brigantepolitico sotto le mentitespoglie del Sacerdote, a p. 2, Dedicato ai contadini e proprietari agricoltori, e a p. 3, Fascio Ital. di combattimento di Lamporecchio. 206. «L'Ordine», a. II, n.11, Pistoia, 12 marzo 1922, 4 pp. Articolo di IT a p. 1, Un nuovo libro. Alle pp. 3-4 articolo su Lamporecchio, Dai comuni del Circondario. Da Lamporecchio.

207. «L'Ordine», a. II, n.13, Pistoia, 19 marzo 1922, 4 pp. Articolo di IT a p. 1, Il pericolo nero. A p. 4, articolo su Lamporecchio, Dai comuni del Circondario. Da Lamporecchio: Fascio di Combattimento di Lamporecchio, firmato da IT, e Conferenza. Della vecchia e della nuova Amm.ne Comunale, in cui IT viene citato tra gli oratori.

208. «L'Ordine», a. II, n. 9, Pistoia, 26 febbraio 1922, 4 pp., 7 copie. Articoli su Lamporecchio a p. 3, Dai Comuni del circondario. Da Lamporecchio, e a p. 4, Fascio Ital. Di Combattimento di Lamporecchio.

209. «Giovinezza. Settimanale di fede e pensiero fascista», a. I, n. 27, Empoli, 27 novembre 1921, 4 pp. Articolo di IT a p. 3, Il problema dell'educazione. 210. «Giovinezza», a. II, n. 28, Empoli, 4 dicembre 1921, 4 pp. Articolo di IT a p. 2, Il problema dell'educazione.

211. «Giovinezza», a. II, n. 31, Empoli, 25 dicembre 1921, 4 pp. Articolo di IT a p. 1, Oggi piu che sempre: Fascisti "A noi". Natale.

212. «Giovinezza», a. II, n. 1, Empoli, 1 gennaio 1922, 4 pp.

213. «Giovinezza», a. II, n. 4, Empoli, 22 gennaio 1922, 4 pp.

214. «Giovinezza», a. II, n. 11, Empoli, 12 marzo 1922, 4 pp.

215. «Giovinezza», a. II, n. 24, Empoli, 11 giugno 1922, 4 pp.

216. «Giovinezza», a. III, n. 2, Empoli, 14 gennaio 1923, 4 pp.

217. «Giovinezza», a. III, n. 3, Empoli, 21 gennaio 1923, 4 pp.

218. «La Nazione», a. LXIV, n. 258, Firenze, 3 novembre 1922, 4 pp.

219. «Avvenire Socialista», a. 1921, n. 8, Pistoia, 17 marzo 1921, 4 pp.

220. «Vita Nuova», Empoli, a. I, n. 11, 1 dicembre 1901, 4 pp.

221. «Vita Nuova», Empoli, 20 maggio 1906, 4 pp. Articolo di IT a p. 1, La rubrica del contadino.

222. «Vita Nuova», Empoli, a. VI, n. 268, 4 novembre 1906, 4 pp. Articolo di IT a p. 1, 2 novembre.

223. «Vita Nuova», Empoli, a. XII, n. 489, 29 gennaio 1911, 4 pp.

224. «Vita Nuova», Empoli, 20 aprile 1913,4 pp. Poesia di IT a p. 1, Resurrezione.

225. «La Sementa. Giornale socialista della provincia», a. V, n. 20, Lucca, 1617 luglio 1904, 4 pp.

226. «La Ragione. Giornale socialista delle Puglie», a. II, n. 53, Bari, 6 aprile 1902, 4 pp.

227. «Il Risveglio della Montagna. Numero unico illustrato», Sambuca Pistoiese, dicembre 1907, $4 \mathrm{pp}$.

228. «Il Lavoratore. Giornale dei Socialisti Italiani in Austria», a. XIX, n. 2397, 15 marzo 1913, 4 pp. 
229. «Il Popolo Pistoiese. Periodico politico-amministrativo di Pistoia e Circondario», a. XLI, n. 13, Pistoia, 29 marzo 1919, 4 pp. Articolo di IT a p. 1, In difesa del socialismo.

230. «La Riscossa. Organo della Federazione Provinciale Fascista Fiorentina», a. II, n, 19, Firenze, 13 maggio 1922, 4 pp. Articolo di IT a p. 2, I doveri del contadino per IT Contadino e proprietario hanno interesse a stare uniti-puntata n. 3.

231. «La Riscossa», a. II, n, 25, Firenze, 24 giugno 1922, 4 pp.

232. «Il Grido del Popolo. Organo dei socialisti piemontesi», a. VII, n. 39, 24 settembre 1898, 4 pp.

233. «La Difesa religiosa e sociale», a. XVI, n. 17, Pistoia, 29 aprile 1911, 4 pp.

234. «L'Azione. Settimanale politico del Circondario Pistoiese», a. III, n. 37, Pistoia, 4 ottobre 1924, 4 pp. Articolo di IT a p. 2, Dal Circondario. Da Lamporecchio. Il rinvio dell' inaugurazione del monumento ai Caduti. A p. 3, in Per la Vita de L'Azione, compare il nome di IT come sottoscrittore e promotore della raccolta di fondi in favore del giornale.

235. «La Difesa», a. [?], n. [?], Firenze, s.d. [marzo 1919], 4 pp. A p. 3, Corrispondenze. Lamporecchio. Alla Martinella, articolo di Alfredo Vescovi, consigliere comunale di Lamporecchio tra le fila dei socialisti, che critica un articolo de «La Martinella» in cui viene chiamato in causa e critica l'operato amministrativo e il trasformismo politico di IT.

236. «La Difesa», a. XIV, n. 4, Firenze, 28 gennaio 1911, 4 pp.

237. «Pistoia Nuova. Giornale industriale, politico, amministrativo della Città e del Circondario», a. I, n. 3, Pistoia, 16 ottobre 1909, 4 pp.

238. «La Gioventù socialista», a. VII, n. [?], Roma, 12 luglio 1908, 4 pp.

239. «Battaglie d'oggi», rivista, a. V, n. 18-19, Napoli, 15 ottobre-1 novembre 1909, 16 pp.

240. «La Parola dei Poveri. Foglio di propaganda popolare socialista. Supplemento quindicinale al "Grido del Popolo"», rivista, a. I, n. 15, Torino, 20 giugno $1897,8 \mathrm{pp}$.

241. «La Parola dei Poveri», rivista, a. I, n. 16, Torino, 5 luglio 1897, 8 pp.

242. «La Parola dei Poveri», rivista, a. I, n.27, Torino, 20 dicembre 1897, 8 pp.

243. «La Parola dei Poveri», rivista, a. II, n. 3, Torino, 5 febbraio 1898, 4 pp. (4 pp. mancanti).

244. «La Parola dei Poveri», rivista, a. II, n. 4, Torino, 20 febbraio 1898, 4 pp. (4 pp. mancanti).

245. «La Parola dei Poveri», rivista, a. V, n. 3, Torino, 25 dicembre 1900, 8 pp. 246. «La Parola dei Poveri», rivista, a. VI, n. 2, Torino, 25 gennaio 1901, 8 pp. 247. «La Parola dei Poveri», rivista, a. II, n. 6, Torino, 20 marzo 1898, 8 pp. 248. «La Parola dei Poveri», rivista, a. [?], n. [?], Torino, s.d., 4 pp. (4 pp. mancanti). 249. La Parola dei Poveri», rivista, a. [?], n. [?], Torino, s.d., 4 pp. (4 pp. mancanti).

250. «L'Asino», rivista, a. [?], n. [?], s.l., s.d., 1 c., 2 pp. (incompleto). 
251. «Il Razionalista», rivista, a. [?], n. [?], s.l., s.d., 4 pp. (incompleto).

252. «La Cultura Socialista. Rivista quindicinale», rivista, a. I, n. 6, Roma, 16 marzo 1908, $16 \mathrm{pp}$.

253. «L'Aurora del $1^{\circ}$ Maggio», numero unico, Milano, 1 maggio 1904, 4 pp. 254. «Critica Sociale. Rivista quindicinale del Socialismo», rivista, a. XXIV, n. 13, Milano, 1-15 luglio 1914, 16 pp.

255. «Numero Unico stampato a cura della Sezione Socialista di Lamporecchio per le elezioni Amministrative del 12 luglio 1914», Empoli, Tip. Parigi Noccioli, 1914, 4 pp.

256. Cittadini e Contribuenti del Comune di Lamporecchio, manifesto, Lamporecchio, 14 novembre 1907, 2 pp., 4 copie. Manifesto elettorale per le elezioni politiche e amministrative per il comune di Lamporecchio del 1907.

257. Fascio Italiano di combattimento di Lamporecchio - Il Fascismo e le elezioni politiche, manifesto, Lamporecchio, 22 aprile 1921, 2 pp. (di cui una bianca). Si pubblicizza un'orazione sul tema Il Fascismo e le elezioni politiche, durante la quale avrebbero dovuto prendere la parola IT, il marchese Dino Perrone Compagni e l'on. Dino Philipson.

258. Discorso pronunziato dall'on. Casciani alla Camera dei Deputati, nella seduta del 4 Giugno 1902, come Relatore del Bilancio di Agricoltura, Industria e Commercio, carte stampate, Pistoia, Tipo-Litografia Grotta Giusti, 1902, 4 pp.

259. A. Angiolini, Socialismo e Socialisti in Italia, opuscoli a stampa, Firenze, G. Nerrini Editore, 1900, 62 pp.

260. «La Tribuna Illustrata», periodico, numeri 1, 2, 5, 47, s.l., s.d., 26 pp.

261. «Cronache della Civiltà Elleno-Latina», estratto di libro o rivista, s.l., s.d., 14 pp.

262. Comune di Lamporecchio, Relazione del Commissario Prefettizio Avv. Pietro Bianchi sul funzionamento dell'azienda annonaria e dello Spaccio Comunale di Lamporecchio, opuscolo, Pistoia, Stab. Grafico Niccolai, 1921, $24 \mathrm{pp}$.

263. Comune di Certaldo, Conto morale redatto dalla Giunta Municipale relativo all'esercizio finanziario 1903, Certaldo, Tip. Benvenuti, 1904, 12 pp.

\section{Appendice - Elenco dei volumi della biblioteca personale di IT}

Le scatole 6, 7 e 8 contengono i volumi che presumibilmente hanno fatto parte della biblioteca privata di IT. Molti di essi presentano delle sovracopertine protettive, ricavate dalle carte di numerose copie del numero 4 della rivista letteraria «Nuove Pubblicazioni», al cui interno sono presentate, con breve descrizione dei contenuti, quattro opere del Targioni (Canzoniere di poesie sociali, I miei Ricordi d' infanzia, ovvero I proverbi di mamma Giuditta, Ettor e Giovannitti, Piccoli parassiti), tutte edite nel 1912. 


\section{Scatola 6}

\section{Consistenza: 29 op. a st.; 8.253 pp.}

1. Vocabolario Italiano e Latino diviso in due tomi. Ne' quali si contengono le Frasi piu eleganti, e difficili, i Modi di dire, Proverbj, ec. Dell'una, e dell'altra Lingua; con in fine le favole, e i nomi delle principali città, castella, mari, fumi, monti, ecc. Per uso delle Regie Scuole di Torino, Tomo Primo, edito da Pietro Savioni, Venezia, 1777.

2. Emile Zola, L'Assommuàr, s.e., s.l., s.d., 354 pp. Opera tradotta in italiano da P. Petrocchi. Sull'interno della prima pagina di copertina è presente un elenco di parole mss. in corsivo, a lapis, ad opera di IT.

3. L'Italia e i suoi difensori, Angelo Usigli Editore, Firenze, 1860, 166 pp.

4. Eugenio Sue, I misteri di Parigi, Casa Editrice La Cisalpina, Milano, s.d., $952 \mathrm{pp}$.

5. Edmondo De Amicis, Lotte Civili. Raccolta di Bozzetti, Scritti e Conferenze Socialistiche, E. Nerbini Editore, Firenze, 1899, 224 pp.

6. Maurizio Basso, Giuseppe Verdi. La sua vita, le sue opere, la sua morte, Società Editrice 'La Milano' di G. Corsi \& C., Milano, 1901, 266 pp.

7. Alexandre Dumas, Il Conte di Monte Cristo, Società Editrice Sonzogno, Milano, 1906, 539 pp.

8. Jessie W. Mario, Della vita di Giuseppe Mazzini. Opera illustrata con ritratti e composizioni d'insigni artisti, Società Editrice Sonzogno, Milano, 1896, 500 pp. A p. X è presente la seguente iscrizione ms. a lapis: «Libro della Vita di Mazzini Giuseppe/ Niccoli Cesare/ Vaiano/ 13/11/ 96».

9. Emilio Salgari, Al Polo Nord. Avventure illustrate da 19 disegni di G. Gamba, A. Donath Editore, Genova, 1899, 256 pp.

10. L'Odissea d'Omero recata dal Greco esametro nell'ottava rima italiana da Lorenzo Mancini, Tomo II - Parte I, Stamperia Mariano Cecchi, Firenze, $1847,280 \mathrm{pp}$.

11. Emile Zola, Maddalena Férat, Casa Editrice Bietti, Milano, s.d., 272 pp. A protezione del libro è stata collocata una sovracopertina in carta, con autore e titolo mss. in corsivo a lapis.

12. Emile Zola, Germinal o i lavoratori sotterranei, Vol. II, Adriano Salani Editore, Firenze, 1908, 210 pp.

13. Emile Zola, Il Capitano Burle, Salvatore Romano Editore, Napoli, 1909, $144 \mathrm{pp}$

14. Emile Zola, Gli amanti, Salvatore Romano Editore, Napoli, 1909, 144 pp.

15. Opere in prosa di Gio. Bat. Niccolini, Co' Tip del Guasti, Prato, 1841, 336 pp.

16. Ellero o Guyot? Studio critico-sociale dell'avvocato Francesco Armelani, Tip. Editrice della Lente di Osvaldo Paggi, Pitigliano, 1895, 240 pp.

17. Francesco Domenico Guerrazzi, Scritti Vari, Casa Editrice Italiana di M. Guidoni, Torino-Milano, 1862, 148 pp. 
18. Crediamo in Dio! Catechismo popolare con ragioni ed esempi storici pel sacerdote Lodovico Sculler, Libreria Religiosa di A. Saraceni, Roma, 1885, $168 \mathrm{pp}$.

19. Francesco Domenico Guerrazzi, Amelia Calani ed altri scritti, Casa Editrice Italiani di M. Guidoni, Milano, 1868, 288 pp.

20. Poesie varie, [senza casa editrice], s.l., s.d., 336 pp.

21. Scelta storica in forma di cronica toscana compilata sopra ricordano Malespini Dino, Compagni Giovanni, Matteo e Filippo Villani, Tomo I, Bracali stampatori Vescovili, Pistoia, 1826, 278 pp.

22. Scelta storica in forma di cronica toscana compilata. Sopra ricordano Malespini Dino, Compagni Giovanni, Matteo e Filippo Villani, Tomo II, Bracali stampatori Vescovili, Pistoia, 1826, 278 pp.

23. Tommaso Grossi, Ulrico e Lida, Carlo Schiepatti Libraio, Torino, 1837, $150 \mathrm{pp}$.

24. Giovanbattista Bisso, Introduzione alla volgar poesia in due parti divisa, Gio. Battista Cannetti, Roma, 1807, 518 pp.

25. Bonaventura Mazzarella, Critica della Scienza, Stab. Tipografico L. Lavagnino, Genova, 1860, $552 \mathrm{pp}$.

26. Paolo Mantegazza, Fisiologia del piacere, Vol. I, Attilio Barion Editore, Milano, 1923, 178 pp.

27. Oscar Giacchi, Pazzi e birbanti, Emilio Croci Editore, Milano, 1885, 312 pp.

28. Paolo Mantegazza, Fisiologia del dolore, Attilio Barion Editore, Milano, 1926, $240 \mathrm{pp}$.

29. Francesco Domenico Guerrazzi, Beatrice Cenci. Storia del secolo XVI, Vol. I, Casa Editrice Italiani di M. Guidoni, Milano, 1872, 352 pp.

\section{Scatola 7}

\section{Consistenza: 51 op. a st.; 13.556 pp.}

1. Lev Tolstoj, Resurrezione, Vol. I, Adriano Salani Editore, Firenze, 1901, $254 \mathrm{pp}$.

2. Lev Tolstoj, Resurrezione, Vol. II, Adriano Salani Editore, Firenze, 1901, 224 pp.

3. Rinaldo Poggi, Nuove idee contro la pena di morte, Top. Niccolai, Pistoia, 1884, $92 \mathrm{pp}$.

4. Giordano Bruno, Bestia Trionfante, Edoardo Parino Tipografo Editore, Empoli, 1888, 212 pp.

5. S. Di Montepin [Xavier de Montépin], Il Manicomio d'Auteuil, Casa Editrice Antonio Bietti \& C., Milano, 1907, 254 pp.

6. Lorenzo Stecchetti, Postuma. Canzoniere, Attilio Barion Editore, Milano, 1922, 160 pp. 
7. Emile Zola, Nel paese dei felici, Salvatore Romano Editore, Napoli, 1909, 160 pp.

8. Paolo A. Bocci, La democrazia, senza casa editrice, s.l., s.d., 112 pp.

9. Paolo A. Bocci, L'eroe della carità, s.l., s.d., 276 pp.

10. Felice Cavallotti, I Messenj. Dramma in quattro atti, Carlo Barbini Editore, Milano, 1877, $160 \mathrm{pp}$.

11. Crevier [Jean Baptiste Louis], Storia degli imperatori romani da Augusto fino a Costantino, Tomo I, Costantino Mezzana Tipografo-Editore, Roma, 1835, 190 pp.

12. Crevier, Storia degli imperatori romani da Augusto fino a Costantino, Tomo II, Costantino Mezzana Tipografo-Editore, Roma, 1835, 170 pp.

13. Crevier, Storia degli imperatori romani da Augusto fino a Costantino, Tomo III, Costantino Mezzana Tipografo-Editore, Roma, 1836, 144 pp.

14. Crevier, Storia degli imperatori romani da Augusto fino a Costantino, Tomo IV, Costantino Mezzana Tipografo-Editore, Roma, 1836, 146 pp.

15. Crevier, Storia degli imperatori romani da Augusto fino a Costantino, Tomo V, Costantino Mezzana Tipografo-Editore, Roma, 1836, 142 pp.

16. Niccolò Macchiavelli, Scritti vari, senza casa editrice, s.l., s.d., 128 pp.

17. Arturo Labriola, Riformismo e sindacalismo, Tip. Campolmi e Sevieri, Firenze, 1905, $32 \mathrm{pp}$.

18. Andrea Giannelli, Lettere di Giuseppe Mazzini ad Andrea Giannelli, Tip. del «Popolo Pistoiese», Pistoia, 1892, 576 pp.

19. Jacopo Jozzelli, Raccolta di prose e poesie edite ed inedite, Tip. Edit. Cacialli \& C., Pistoia, 1896, 192 pp.

20. Paolo Mantegazza, Almanacco igienico popolare, Fratelli Treves Editori, Milano, 1900, 168 pp.

21. Paolo Mantegazza, Almanacco igienico popolare, Fratelli Dumolard Editori, Milano, 1890, $160 \mathrm{pp}$.

22. Niccolò Carteromaco, Ricciardetto, Vol. I, Francesco Pitteri Librajo, Parigi, 1763, $420 \mathrm{pp}$.

23. Niccolò Carteromaco, Ricciardetto, Vol. II, Francesco Pitteri Librajo, Parigi, $1763,432 \mathrm{pp}$.

24. Compendio della Storia romana, senza casa editrice, s.l., s.d., 347 pp.

25. Paolo Mantegazza, Almanacco igienico popolare, Fratelli Treves Editori, Milano, 1900, 168 pp. Alle pp. 347-348 è presente la bozza di sonetto ms. in corsivo a lapis.

26. Dizionario delle favole per uso delle scuole d'Italia, Antonio Zatta, Venezia, 1796, $232 \mathrm{pp}$.

27. Camillo Cavour, Libera Chiesa in libero Stato, Società Editoriale Milanese, Milanese, 1909, 96 pp.

28. Massime di Napoleone Primo relativamente alla guerra, senza casa editrice, s.l., s.d., 36 pp. 
29. Critica della teoria del Proudhon sulla proprietà, senza casa editrice, s.l., s.d., 36 pp. (mancanti le pp. 35-70).

30. Roberto Puccini, Nel 2000 ossia del futuro socialismo. Esame critico popolare, Scuola Tipografica [?], Firenze, 189[?], 252 pp.

31. Esempi di bello scrivere in poesia scelti ed illustrati dall'Avv. Luigi Fornaciari, Tipografia Giusti, Lucca, 1840, 318 pp. + 12 pp. (introduzione numerata con caratteri latini). Alle pp. II-III sono presenti scritte a lapis con cui il proprietario del libro annota il suo nome (che risulta illeggibile), specificando che il destinatario del prestito, probabilmente IT, avrebbe dovuto restituirlo.

32. Felice Cavallotti, Agatodémon, Carlo Aliprandi Editore, 1894, 174 pp.

33. Opere di Benedetto Menzini. Contenente varie poesie toscane, Gaspero Ricci, Firenze, 1819, 396 pp. +12 pp. (introduzione numerata con caratteri latini). A p. XII reca l'iscrizione ms. «Targioni».

34. Lev Tolstoj, La sonata a Kreutzer, Adriano Salani Editore, Firenze, 1898, 220 pp. A p. 1 sono presenti calcoli numerici mss. e la scritta corsiva «Targioni Idalberto». A p. 220 presente timbro con inchiostro viola recante la seguente iscrizione: «BIGLIETTO D’INVITO/ Rilasciato al Sig./ Per accedere all'ACCADEMIA poetica / del poeta estemporaneo / IT / nel / questa sera alle ore».

35. Eugenio Comba, Nuovo compendio di Storia d'Italia. Corredato di esercizi d'applicazione ad uso delle scuole tecniche, normali e magistrali. Parte I - Storia Romana, Ditta G. B. Paravia e Comp., Roma-Torino-MilanoFirenze, 1883, 302 pp. Le pagine precedenti a p.1 e quelle successive a p. 302 presentano scritte e disegni probabilmente realizzati da IT.

36. La Iliade d'Omero recata dal testo greco in versi toscani dall'Abate Melchior Cesarotti, Vol I-II, Stamperia di Giacomo Fea, Torino, 1790-1795, pp. $402+356$.

37. Paolo Mantegazza, Fisiologia dell'Amore, Attiolio Barion Editore, Miano, 1922, 192 pp.

38. Ercole Ricotti, Breve storia d'Europa e specialmente d'Italia dall'anno 476 al 1861, G. B. Paravia e Comp. E V. Maisner e Comp. Coeditori, Torino-Milano, febbraio 1876, $672 \mathrm{pp}$.

39. Vincenzo Monti, Poesie Liriche, Adriano Salani Editore, Firenze, 1891, $224 \mathrm{pp}$.

40. L'Odissea di Omero tradotta in versi italiani da Francesco Soave con annotazioni aggiuntavi "La Batracomiomachia", Tomo II, Pietro Galeazzi, Pavia, 1805,308 pp. A p. 1, sul frontespizio, è presente la seguente iscrizione ms.: «Proprietà di Targioni Idalberto $-\mathrm{S}$. Baronto / Lamporecchio».

41. Victor Hugo, Il novantatré, Carlo Simonetti Editore, Milano, 1884, 304 pp.

42. A. Della Pura, Antologia di poesie [frontespizio mancante], Bemporad Editore, Firenze, 1881, 256 pp. Sugli interni della copertina presenti 
iscrizioni mss. Sull'interno dell'ultima di copertina è presente la scritta «Targioni».

43. Silvio Pacini, I fatti della storia italiana. Prima Parte - Storia Romana, Felice Paggi Editore, Firenze, 1885, 276 pp. +8 pp. di postfazione (con numerazione con caratteri latini).

44. Giuseppe Rigutini, Elementi di retorica compilati per uso delle scuole italiane, Felice Paggi Editore, Firenze, 1887, 444 pp. +8 pp. di prefazione e $12 \mathrm{pp}$. di postfazione (con numerazione con caratteri latini).

45. La Divina Commedia di Dante Alighieri. Con note di Paolo Costa. Vol. I-L'Inferno, Casa Editrice Italiana di M. Guidoni, Miano, 1873, 356 pp. Sull'interno dell'ultima di copertina è presente la seguente iscrizione ms. «Amelia Targioni».

46. Paolo Mottura, Nuova antologia di prose e poesie italiane. Compilata secondo i Programmi governativi per uso delle Scuole Ginnasiali Superiori e degli Istituti Tecnici, G. B. Paravia e Com., Firenze-Torino-Milano, $1866,304 \mathrm{pp}$. Sul verso dell'ultima carta celeste, collocata prima dell'ultima di copertina, è presente una bozza di lettera ms. destinata a Giulia Servaddio.

47. F. Galvani, Raccolta degli avvenimenti piu importanti che segnalarono $i$ primordi della vita politica di S. M. Umberto I, Re d'Italia, fino al 7 marzo 1878, Stab. Benedetto Sborgi, Firenze, 1878, 192 pp.

48. Carlo Hegel, Storia della costituzione dei municipi italiani dai Romani al chiudersi del secolo XII, Casa Editrice Italina Maurizio Guigoni, MilanoTorino, 1861, $720 \mathrm{pp}$.

49. Enrico Morselli, Carlo Darwin e il Darwinismo nelle scienze biologiche e sociali. Scritti vari, Fratelli Dumolard Editori, Milano, 1892, 302 pp.

50. Raccolta dei numeri 1-26 (anno I) della rivista «Letture utili e amene. Polimazia di famiglia» (1853-1854) e dei numeri 1-18 (anno I) della rivista «L'Utile. Giornale scientifico artistico industriale e morale» (Lucca, 1858-1859), 384 pp. + 144 pp.

51. Paolo Costa, Della elocuzione e dell'arte poetica, Tipografia di Pietro Fraticelli, Firenze, 1845, 226 pp.

\section{Scatola 8}

Consistenza: 117 op. a st.; 33.391 pp.

1. Gian Pietro Lucini, Revolverate (Con una prefazione di F. T. Marinetti), libro, Milano, Off. Grafica Bertieri e Vanzetti, 1909, 360 pp.

2. Teofilo Gay, La terra del Cristo. Viaggio in Oriente, opuscolo, Napoli, Tip. Ferrante, 1878, 98 pp.

3. Victor Hugo, L'ultimo giorno di un condannato a morte, Facchi Editore, Milano, s.d., 224 pp. 
4. Luigi Onorato, Ricordi dell' imperatore Marc'Aurelio, G. Barbera Editore, Firenze, 1867, 406 pp.

5. Poesie Scelte di Giovanni Prati, a cura di Ferdinando Martini, G. C. Sansoni Editore, Firenze, 1892, 374 pp.

6. Opere Volgari di Messer Angelo Ambrogini Poliziano, a cura di Tommaso Casini, G. C. Sansoni Editore, Firenze, 1892, 376 pp.

7. Leopere minori di Dante Alighieri novamente annotate da G. L. Passerini, Vol. V-Il trattato della volgare eloquenza, G. C. Sansoni Editore, Firenze, 1912, 172 pp.

8. Cornelio Tacito, La vita di Giulio Agricola, G. C. Sansoni Editore, Firenze, $1892,232 \mathrm{pp}$.

9. Anna Vertua Gentile, Michela, A. Barion Editore, Milano, 1921, 200 pp.

10. Anna Vertua Gentile, Un terrazzo fra due case, A. Barion Editore, Milano, 1921, $168 \mathrm{pp}$.

11. Anna Vertua Gentile, L'odio di Rita, A. Barion Editore, Milano, 1920, 222 pp., 2 copie.

12. W. Shakespeare, Giulietta e Romeo, Facchi Editore, Milano, s.d., 222 pp.

13. Florence Montgomery, Incompreso, A. Barion Editore, Milano, 1923, 254 pp.

14. Gabriele D'Annunzio, L'invincibile, Facchi Editore, Milano, 1923, 222 pp.

15. Galileo Galilei, Il Saggiatore, G. Barbera Editore, Firenze, 1864, 556 pp.

16. Tommaso Campanella, La città del Sole, Oreste Garroni Editore, Roma, 1910, 92 pp.

17. Lorenzo de' Medici, Poesie, G. Barbera, Firenze 1859, 462 pp.

18. Matilde Serao, L'infedele, A. Barion Editore, Milano, 1921, 200 pp.

19. Enrico Bianchi, Dizionarietto di mitologia e di coltura classica, G. C. Sansoni, Firenze, s.d., 380 pp.

20. Neera, Addio!, A. Barion Editore, Milano, 1922, 222 pp.

21. Paolo Mantegazza, L'arte di campar vecchi, A. Barion Editore, Milano, 1901, 216 pp.

22. Giosuè Carducci, Primavera e fiore della Lirica Italiana, G. C. Sansoni, Firenze, 1904, 410 pp. A p. 410 e sull'interno dell'ultima di copertina presente bozza ms.

23. M. T. Cicerone, Le catilinarie, G. C. Sansoni, Firenze, 1927, 136 pp.

24. Giovanni Verga, Storia di una capinera, A. Barion Editore, Milano, 1922, $280 \mathrm{pp}$.

25. Luciano Zuccoli, Il maleficio occulto, A. Barion Editore, Milano, 1922, $222 \mathrm{pp}$.

26. Paolo Costa, Della elocuzione della sintesi e dell'analisi, Tip. Felice Le Monnier, 1837, $160+64$ pp. di postfazione.

27. Giorgio Vasari, Capricci e aneddoti di artisti, G. Barbera, Firenze, 1872, $472 \mathrm{pp}$. 
28. Francesco Mario Pagano, Discorso sull'origine e natura della poesia e saggio del gusto e delle belle arti, Tip. Ruggia e C., Lugano, 1832, 164 pp.

29. Francesco Maria Zanotti, Filosofia morale secondo l'ordine dei peripatetici, G. Barbera, Firenze, 1882, 152 pp.

30. Angelo Marini, I papi e l'Unità d'Italia, Tip. M. Cellini e C., Firenze, $1883,104 \mathrm{pp}$.

31. Tommasina Guidi, Onestà di donna, A. Baron Editore, Milano, 1919, $160 \mathrm{pp}$.

32. Francesco Pera, Affetti e virtù. Letture per le famiglie e per le scuole, Felice Paggi Editore, Firenze, 1871, 310 pp.

33. P. Anastasio Bocci, La democrazia e la questione sociale, Vol. II, E. Ariani Editore, Firenze, 1893,112 pp. Sull'ultima pagina sono presenti annotazioni mss. con elenco di nomi e relative cifre corrispondenti.

34. P. Anastasio Bocci, La democrazia e la questione sociale, Vol. III, E. Ariani Editore, Firenze, 1893, 120 pp.

35. Gianbattista Casti, Gli animali parlanti. Poema Epico, senza casa editrice, s.l., 1848, 244 pp.

36. Luigi Scappucci, Versi, Tip. Niccolai, Pistoia, 1879, 170 pp.

37. Pietro Gori, L'amore per i fiori, Adriano Salani Editore, Firenze, 1894, 334 pp.

38. Ludovico Ariosto, I promessi sposi. Ruggiero e Bradamante, Tip. della Gazzetta d'Italia, Firenze, 1875, 334 pp.

39. Pietro Lancetta, Sunto di lezioni di Scienze naturali e d'igiene, Tip. Lucri e Secagni e Figli, Modica, 1876, 130 pp.

40. P. Anastasio Bocci, Opere Varie, Casa Editrice Guigoni, Milano, 1885, $238 \mathrm{pp}$. A protezione del libro è stata collocata una sovracopertina, ricavata da una carta estratta da una copia del numero 4 della rivista letteraria «Nuove Pubblicazioni».

41. Enrico Matcovich, Album d'uno scapato, Enrico Politti Editore, Milano, s.d., 160 pp.

42. Salvatore Farina, Dalla spuma del mare, Libreria Editrice G. Brigola, Milano, 1876, $218 \mathrm{pp}$.

43. Ida Baccini-Ferri, Frutti fuori stagione, Tip. Carnesecchi e figli, Firenze, 1871, 112 pp.

44. Guido Podrecca, Materialisti e spiritualisti, Luigi Mongini Editore, Roma, 1904, 74 pp.

45. Le favole di Fedro. Ridotte ognuna in un'ottava da Giuseppe Riva, Tip. A. Bianchi, Padova, 1872, 70 pp.

46. Giuseppe Signorini, Nozioni di Morale, Società editrice Dante Alighieri, Roma, 1897, $164 \mathrm{pp}$.

47. F. M. Pasanisi, L'Europa. Testo di Geografia per la $5^{\circ}$ Ginnasiale, Società editrice Dante alighieri, Roma, 1894, 160 pp.

48. Teodolinda Franceschi Pignocchi, Nuove rime, Tip. Fava Garagnani, Bologna, 1873, 136 pp. 
49. Iacopo Bordoni, A colpi di martello, Tip. Roberto Lastrucci, Firenze, 1902, 194 pp.

50. Il deputato italiano, senza casa editrice, s.l., s.d., 142 pp.

51. Opera edita incompleta con raccolta di poesie, senza casa editrice, s.l., s.d., 44 pp. (molte pagine mancanti).

52. Poesie di Teresa Gnoli, senza casa editrice, s.l., s.d., 86 pp.

53. Ludovico Canonico Schuller, Il futuro e le scoperte meravigliose, Libreria Religiosa di Antonio Saraceni, Roma, 1889, 190 pp.

54. F. Romolo da Pistoia, La filosofia del moderno epicureismo e la barbarie eterodossa del moderno protestantesimo, Tip. Vescovile, Pistoia, 1878, 120 pp.

55. Alfredo Pasquali, Sonetti popolari, Tip. del 'Popolo Pistoiese', Pistoia, $1890,78 \mathrm{pp}$.

56. V. De Fereal, L'inquisizione di Spagna. Romanzo storico, Casa Editrice Nerbini, Firenze, s.d.

57. Emilio Dandolo, I volontari ed i Bersaglieri lombardi. Annotazioni storiche, Tip. Ferrero e Franco, Torino, 1849, 304 pp.

58. Lorenzo Luchetti, Improvvisazioni e giudizi di periodici italiani sull'autore, Tip. Niccolai, Pistoia, 1893, 64 pp. +8 pp. di postfazione. A p. 64 è presente la seguente iscrizione $\mathrm{ms.:}$ « $\mathrm{Al}$ mio genero Tranquillo Gasperini per ricordo offro/ L. Lucchetti».

59. Felice Cavallotti, Poesie scelte, Società Editrice Sonzogno, Milano, 1897, $112 \mathrm{pp}$.

60. G. B. Niccolini, Opere, Vol. III, Felice Le Monnier, Firenze, 1847, 430 pp.

61. Jules Verne, Dalla Terra alla Luna. Tragitto in 97 ore e 20 minuti, Fratelli Treves Editori, Milano, 1878, 162 pp.

62. Orazio Antinori, Nel centro dell'Africa, Edoardo Perino Editore, Roma, 1884, 96 pp.

63. G. Giannetti, Precetti di retorica, senza casa editrice, s.l., s.d., 176 pp.

64. Poesie e prose inedite o rare di italiani viventi, Vol. I, Tip. dell'Aquila, Bologna, 1835, 169 pp.

65. Giovan Santi Saccenti, Rime, G. Galletti, Firenze, 1830, 284 pp.

66. Miscellanea.

a. Differenza fra il Protestantismo e il romanismo o papismo, stamperia Sarpiana, Firenze, 1861, 24 pp.

b. Storia di Andrea Dunn, Tip. Claudiana, Firenze, 1864, 96 pp.

c. La vendetta, Tip. Claudiana, Firenze, 1867, 152 pp.

d. La credulità degli increduli, Tip. Claudiana, Firenze, 1865, 62 pp.

e. Quadri evangelici, Tip. Claudiana, Firenze, 1870, 152 pp.

f. Un primo albero di natale, Tip. Claudiana, Firenze, 1870, 32 pp.

g. Code de jesuites, Wouters e Comp., Bruxelles, 1845, 108 pp.

h. La custode del crocefisso, Tip. Claudiana, Firenze, 1871, 8 pp.

67. Giovanni Fantoni, Poesie, Luigi Vannini, Prato, 1820, 180 pp. 
68. Vittorio Alfieri, Teatro tragico originale, Vol. II, senza casa editrice, s.l., 1809,306 pp. Nelle ultime pagine sono presenti annotazioni mss.

69. Miscellanea di poesie di vari autori, senza casa editrice, s.l., s.d., 386 pp. Presenti numerose annotazioni mss.

70. Alberto Orsi, La coppia umana, Casa Editrice Madella, Sesto San Giovanni, 1917, $160 \mathrm{pp}$.

71. Virginia Paganini, La scienza spirituale attraverso i secoli, Tip. Cooperativa, Firenze, 1895, 170 pp.

72. Paolo Mantegazza, L'anima delle cose, STEN, Torino, 1910, 392 pp.

73. Alfonso de Lamartine, Rimembranze di un viaggio in oriente, Vol. III, Ditta Pirotta e C., Milano, 1834, 328 pp.

74. Giosuè Carducci, Nuove poesie, Zanichelli, Bologna, 1881, 224 pp.

75. Tragedie di Eschilo. Tradotte da Felice Cavallotti, Tomo I-II-III, La Vedova di Reale e Figli, Napoli, 1830, 168 pp. + 144 pp. + 120 pp.

76. Collezione storica di tutti gli atti, documenti, dibattimenti difese e sentenza della celebre causa di lesa maestà contro F. D. Guerrazzi, Gius. Montanelli, Gius. Mazzoni, Parte II sezione I, Tip. Mariano Cecchi, Firenze, 1852, $886 \mathrm{pp}$.

77. Giuseppe Baretti, Scritti scelti inediti o rari, Vol. I, G. B. Bianchi e C., Milano, 1822, 410 pp.

78. Giuseppe Baretti, Scritti scelti inediti o rari, Vol. II, G. B. Bianchi e C., Milano, 1823, 408 pp.

79. Melchiorre Gioja, Nuovo Galateo, Casa Editrice Guigoni, Milnao, 1886, $436 \mathrm{pp}$.

80. F. D. Guerrazzi, La battaglia di Benevento, Casa Editrice Guigoni, Milano, $1868,192 \mathrm{pp}$.

81. L'aureo trattato di Dioniso Longino intorno al sublime modo di parlare e di scrivere. Tradotto dal greco da Anton Francesco Gori, F.lli Bassaglia, Venezia, 1782, 120 pp.

82. Francesco Petrarca, Il Canzoniere, Giuseppe Antonelli Editore, Venezia, 1832, $304 \mathrm{pp}$.

83. Cesare Balbo, Delle Speranze d'Italia, Felice Le Monnier, Firenze, 1855, $512 \mathrm{pp}$.

84. 200 sonetti ed altre poesie italiane scelte, Edoardo Ducci, Firenze, 1880, $352 \mathrm{pp}$.

85. Barone X, Fango finanziario, Casa Ed. Alberto Costa, Milano, 1892, 256 pp.

86. Filippo Virgilii, Almanacco del nuovo agricoltore, R. Bemporad Editori, 1901, $128 \mathrm{pp}$.

87. Novelle, senza casa editrice, s.l., s.d., 160 pp.

88. Schobert, Povera Regina, Adriano Salani Editore, Firenze, 1911, 260 pp.

89. C. Morand, Dialoghi francesi-italiani, Francesco Pagnoni Editore, Milano, 1861, 316 pp. 
90. Dante Alighieri, La Divina Commedia. Il Paradiso. Con note di Paolo Costa, Casa Editrice Guigoni, Milano, 1873, 408 pp.

91. Giacomo Leopardi, Le prose, Edoardo Perino Editore, Roma, 1888, 262 pp. Sull' interno della prima di copertina è presente la seguente iscrizione ms.: «Comprato da IT a Pistoia il giorno 15 maggio 1892».

92. Virgilio, L'Eneide, Casa Editrice Guigoni, Milano, 1885, 344 pp. Sul frontespizio è presente la seguente iscrizione corsiva fatta con inchiostro nero: «IT».

93. Dialogo contra i poeti, senza casa editrice, s.l., s.d., 336 pp.

94. Ugo Foscolo, Ultime lettere di Jacopo Ortis e discorso sul testo della Commedia di Dante, Edoardo Sonzogno editore, Milano, 1887, 388 pp. Presente annotazione ms. nelle ultime pagine del volume.

95. Carlo Botta, Storia della guerra americana, Tomo I-II-III, Stamperia Formigli, Firenze, 1825, $228+240+228$ pp.

96. Carlo Botta, Storia della guerra americana, Tomo IV-V-VI, Stamperia Formigli, Firenze, 1825, $236+228+288$ pp.

97. Carlo Botta, Storia della guerra americana, Tomo VII-VIII-X, Stamperia Formigli, Firenze, 1825, $226+256+238$ pp.

98. Riguccio Galluzzi, Istoria del Granducato di Toscana sotto il governo della S. Medici, Tomo II-III-IV, Gaetano Ducci, 1830, 198+ $234+212$ pp.

99. Riguccio Galluzzi, Istoria del Granducato di Toscana sotto il governo della S. Medici, Tomo V-VI-VII, Gaetano Ducci, 1830, $220+204+200$ pp.

100. Riguccio Galluzzi, Istoria del Granducato di Toscana sotto il governo della S. Medici, Tomo VIII-IX-X-XI, Gaetano Ducci, 1830, $200+216+220$ pp.

101. Riguccio Galluzzi, Istoria del Granducato di Toscana sotto il governo della S. Medici, Tomo XII-XIII-XIV, Gaetano Ducci, 1830, 220 pp. + $212+$ 208 pp.

102. Riguccio Galluzzi, Istoria del Granducato di Toscana sotto il governo della S. Medici, Tomo XV-XVI-XVII, Gaetano Ducci, 1830, $208+198+208$ pp.

103. Riguccio Galluzzi, Istoria del Granducato di Toscana sotto il governo della S. Medici, Tomo XVIII-XIX-XX-XXI, Gaetano Ducci, 1830, $190+178$ $+76+26$ pp. di postfazione (con numerazione latina).

104. Cesare Lombroso, Delitti Vecchi e delitti nuovi, Fratelli Bocca, Torino, 1908, 336 pp.

105. Emanuele Sella, Monteluce, Zanichelli, Bologna, 1909, 168 pp.

106. Emile Zola, Amori liberi, Salvatore Romano Editore, Napoli, 1906, 230 pp.

107. Nicola Venette, Anatomia e fisiologia dell'amore, OET, Roma, s.d., 162 pp. 108. Giovanni Macé, Storia di un boccone di pane, Milano, editori della Biblioteca Utile, Milano, 1864, 276 pp. Presenti calcoli matematici e parole mss. probabilmente ad opera di IT. 
109. Teresa Venzick, La monaca di Cracovia, Gennaro Monte Libraio, Napoli, 1910, $94 \mathrm{pp}$.

110. Scritti vari di Pietro Verri, Vol. I, Felice Le Monnier, Firenze, 1854, 580 pp.

111. G. B. Niccolini, Filippo Strozzi, Felice Le Monnier, Firenze, 1847, 368 pp.

112. Opere di G. B. Niccolini, Felice Le Monnier, Firenze, 1847, 498 pp.

113. Vittorio Alfieri, Tragedie, Vol. I, Pirotta e Maspero Editori, Milano, 1801, $324 \mathrm{pp}$.

114. Vittorio Alfieri, Tragedie, Vol. II, Pirotta e Maspero Editori, Milano, 1801, $302 \mathrm{pp}$.

115. Vittorio Alfieri, Tragedie, Vol. III, Pirotta e Maspero Editori, Milano, $1801,340 \mathrm{pp}$.

116. Vittorio Alfieri, Tragedie, Vol. IV, Pirotta e Maspero Editori, Milano, 1801, $358 \mathrm{pp}$.

117. Vittorio Alfieri, Tragedie, Vol. V, Pirotta e Maspero Editori, Milano, 1802,348 pp. 
Appendice 



\section{Targioni in privato. Una testimonianza}

di Simonetta Chiappini

Ho conosciuto le affascinanti vicende del 'rosignuolo' Targioni da sua figlia Velia, mia nonna. Anche da vecchia ne parlava spesso, con occhi da innamorata. Me lo descriveva quando arrivava da Firenze in calesse per cantare in ottava, col garofano rosso all'occhiello e la voce tonante di baritono.

Le donne sciamavano intorno a quest'uomo singolare: trovatello, socialista, poeta dall'impetuosa eloquenza. Ma solo lei lo sapeva accogliere per quello che era: un uomo solo, tormentato, infelice. L'immagine del Targioni che mi sono costruita da bambina proveniva da un mondo che non lo aveva ancora del tutto dimenticato. Era quella eroica del poeta contadino, del politico difensore dei miseri, dell'autodidatta divenuto giornalista. 'La nipote del Targioni' mi chiamavano ammirati quelli che ancora sapevano a memoria i suoi versi. Mia nonna non nascondeva il disordine della sua vita sentimentale, quanto fosse stato amato e odiato e come il suo carattere impulsivo e fragile lo rendesse facile preda di illusioni e poi di amare disillusioni. Sempre alla ricerca della mamma, che era stata costretta ad abbandonarlo, ne ripercorreva le tracce, e la figlia affettuosamente lo accompagnava nei soggiorni montani a Casa Marconi, dalla prima famiglia affidataria, dove il 'diavolo rosso' trovava pace: «Val più l'ombra di un castagno vecchio che la Fiera e il Fierin di Lamporecchio».

E alla fine l'irriducibile anticlericale, mi confidava la nonna, aveva chiesto il prete, in punto di morte. Incoerenze, forse, su cui lei passava sopra, decantando del padre sempre e soprattutto l'integrità morale, che gli aveva procurato infiniti tormenti e un'assidua povertà.

Tre anni fa, dopo la morte di mia zia (figlia di Velia e sorella di mio padre), che fungeva da custode e vestale delle vicende familiari, ho trovato tra le sue cose un'inaspettata messe di documenti, lettere, scritti, libri, che mi ha fatto immergere nella ricchezza e complessità dell'esperienza umana e culturale del mio bisnonno Targioni. Mi ero forse liberata dalla infastidita disattenzione con cui spesso, da giovani, guardiamo le narrazioni e le retoriche familiari, e 
ho considerato con occhio più sgombro le testimonianze di una vita singolare che emergevano da quelle carte. Non è stata cosa facile: ho dovuto misurare lo scarto che intercorre tra la memoria infantile e ciò che affiora dal passato, fissato sulla carta, inerme di fronte allo sguardo di chi legge. La prima cosa che mi ha colpita sono stati i documenti in cui Targioni, dopo avere aderito al fascismo, in qualità di segretario del Fascio di Combattimento di Lamporecchio, raccoglieva abiure e sollecitava adesioni al partito mussoliniano da parte degli ultimi socialisti rimasti sul territorio. Avevo saputo del suo passaggio da una sponda all'altra delle parti politiche, ne ero stata vagamente sorpresa e avevo sorvolato. Ma i documenti parlano e ne sono rimasta ferita: non immaginavo che il ribelle, più volte imprigionato per il suo credo socialista, fosse divenuto un 'voltagabbana', acceso fautore dell'ordine e della violenza politica. Su questo discusso e tormentato voltafaccia si interrogò più volte Targioni stesso, cercando e dando motivazioni, personali e politiche, su cui è importante indagare, non solo per sciogliere uno dei nodi fondamentali della sua vicenda privata, ma anche per interrogarci oggi, a quasi un secolo di distanza, su uno dei passaggi più significativi della storia italiana del secolo scorso.

Proprio affrontando questa spinosa questione si apre una breve autobiografia che Targioni scrisse a 52 anni, lasciandola dattiloscritta e incompiuta. La lettura di questo documento è stata una delle più affascinanti scoperte riserbatami dal lascito familiare; sono pagine senza particolare pregio letterario, in cui Targioni ripercorre la sua storia a partire dalla sciagurata infanzia di 'bastardo' dell'Ospedale degli Innocenti. Ne emerge il quadro aspro e commovente di un mondo contadino misero e feroce, ma a suo modo vivo. Targioni descrive l'odissea degli affidi, la reclusione patita nella permanenza all'Istituto, le fatiche del mondo contadino, i difficili rapporti con i coetanei e qualche incontro fortunato, come quello con 'mamma Giuditta', che lo accoglie con amore sapiente e indistruttibile. È lei che cerca di placare le sue rabbie, le asprezze di un carattere risentito e talvolta violento e a lei lui dedica poi versi riconoscenti.

Targioni si descrive come un personaggio romantico, in perenne rissa col mondo esterno, onesto ma irascibile e vendicativo, agitato fin da piccolo da smania di libertà e di grandezza, con l'animo ribelle alle ingiustizie, precocemente e spontaneamente portato al verso.

L'autobiografia si ferma a quando Idalberto torna a San Baronto da mamma Giuditta, dopo avere svolto il servizio militare, intorno ai ventitré anni. Gli anni precedenti erano stati segnati da peregrinazioni che ricordano le peripezie di giovani picari come Huckleberry Finn o Tom Sawyer. Contadino renitente, guardiano di pecore, più tardi manovale alla costruzione della ferrovia faentina, a momenti dominato anche dal demone del gioco, Targioni era comunque invincibilmente attratto verso la poesia, i libri e la cultura, tanto da divenire, analfabeta fino a dieci anni, poeta, giornalista, sindaco, politico «educatore ed organizzatore delle masse contadine», come amava definirsi orgogliosamente, promuovendo l'immagine di uomo che 'si è fatto da solo'. 
I libri erano stati il suo strumento di emancipazione, il suo tesoro. La sua biblioteca era vasta e varia, come non avrei mai potuto immaginare. Alcuni vecchi tomi li conoscevo già, avevo letto il Conte di Montecristo in un'edizione illustrata del primo Novecento, pagine gialle e una traduzione stantia che mi aveva affascinata, c'erano anche $I$ misteri di Parigi di Sue con immagini paurose che avevano tanto spaventato mia zia da bambina, e poi i romanzi russi e francesi che mia nonna leggeva avidamente allattando i figli, amante dei libri anche lei, costretta dal padre anticlericale a interrompere gli studi alla terza elementare, dato che l'unica scuola a San Baronto era gestita dalle suore di Spicchio. Ma nel lascito ho scoperto altri tesori di cui faccio qui un parzialissimo resoconto: gli strumenti del suo mestiere letterario, dai vocabolari del Settecento ai più moderni rimari, compendi letterari, opere rare, come il poema burlesco del pistoiese Niccolò Forteguerri (Carteromaco) Ricciardetto, del 1763. Non mancano i grandi classici: l'Iliade, l'Odissea, la Divina Commedia, l'Orlando Furioso fino alle poesie di Petrarca, Poliziano, Foscolo, Monti, Leopardi (le prose), Giusti, Prati, Carducci. E poi Eschilo, Shakespeare fino ad arrivare ad Alfieri, Cavallotti, Niccolini; vari saggi di estetica e, altra sorpresa, il trattato Sul sublime di Longino.

Le radici della sua formazione affondano nella cultura risorgimentale e positivistica; da una parte i numi tutelari del risorgimento laico come Mazzini, i romanzi storici di Guerrazzi, i versi del Grossi, e poi Verdi, Cavour, Balbo; dall'altra numerosi romanzi francesi naturalisti, e soprattutto Zola; inoltre testi di fisiologia come l'amatissimo (anche da Velia) Mantegazza, Lombroso e un interessante saggio sul darwinismo dello psichiatra Enrico Morselli. Nel versante politico a lui contemporaneo troviamo Turati, Costa, Arturo Labriola, scritti sociali di De Amicis, un saggio su Proudhon, ma non Marx, lacuna interessante, che rivela l'assetto teoreticamente precario del socialismo di Targioni. Non mancano invece opere di filosofi di cultura laica come Tommaso Campanella, Giordano Bruno, Galileo; di illuministi come Baretti, Verri, Melchiorre Gioja e Francesco Mario Pagano. Ampio spazio della biblioteca è dedicato alla storia patria, tra gli altri compaiono Dino Compagni, le Croniche dei Villani, Machiavelli, Vasari, la settecentesca Istoria del Granducato di Toscana sotto il governo della S. Medici, di Riguccio Galluzzi in XXI tomi; Gli interessi di Targioni comprendevano anche la Storia della guerra d'indipendenza degli Stati Uniti d'America di Carlo Botta del 1809 e la Storia degli Imperatori romani da Augusto sino a Costantino dello storico francese di fine Seicento Jean Baptiste Louis Crevier. Mi ha incuriosito la presenza di varie autrici donne: da Florence Montgomery, autrice di Incompreso, alla saggista Virginia Paganini, a giornaliste o romanziere note come la Serao, Neera e meno note, come Anna Vertua Gentile, Tommasina Guidi e Ida Baccini-Ferri. Compaiono anche poetesse e improvvisatrici: Teresa Gnoli e Teodolinda Franceschi Pignocchi.

La lettura di tante lettere, appunti e scritti di varia natura ha confermato la mia convinzione che il tema dell'abbandono sia stato il nodo fondamenta- 
le della sua vita inquieta. Colpisce un parallelo che Targioni stesso istituisce, all'inizio dell'autobiografia, tra l'idea socialista e l'immagine di una donna amata, adorata e idealizzata, che si rivela poi, con classica dicotomia misogina, una «volgare sgualdrina dominata dalle più basse passioni». Non fu lui ad abbandonare il socialismo, quindi, ma il socialismo abbandonò lui, lo tradì, lo lasciò languire in prigione, come la mamma lasciò lui - aggiungo io. Anche in questo senso il rapporto con le donne fu centrale nella vita del Targioni, a partire dalla mamma biologica, immaginata come una giovane donna di modesta condizione sedotta da un anziano principe, sempre cercata, sempre invocata, mai trovata, alla mamma adottiva Giuditta, alla prima moglie Amelia, semplice contadina analfabeta, presto abbandonata, alla più giovane compagna Sofia, alle altre donne amate, e poi alle figlie, legittime o meno. Scorrendo la consistente corrispondenza tra padre e figlia appare evidente che Targioni non esitava a consegnare a Velia tutto intero il suo fardello di malinconie e inquietudini, malesseri e malattie. Le scriveva affettuosamente da tutta Italia, dalla Svizzera, dalla Francia, dove lo portava la sua vocazione di conferenziere in ottava rima e di politico, quasi sempre sfogando sulla figlia le sue frustrazioni per la salute malferma, per affari balordi finiti male, per il lavoro al sindacato mal pagato e poco considerato. Ma la giovane Velia, dal canto suo, era relegata a Lamporecchio, con tre figli piccoli da badare, un bilancio familiare non sempre in attivo e un matrimonio non troppo felice. Alla fine della sua vita, poi, il padre le affida anche Renzo, forse altro figlio illegittimo, e soprattutto le consegna la responsabilità della sua nuova famiglia fiorentina, della compagna Sofia e di sua figlia Idalberta (Albertina), che sarebbero rimaste sole e con scarsi mezzi dopo la sua morte. Nonostante la diffidenza iniziale, che il padre le rimproverava, Velia le accoglie e le sostiene entrambe, tanto che tra i suoi tre figli e Albertina nasce un profondo legame di amicizia, che sarebbe durato tutta la vita. Mi ricordo che quando le due donne venivano da Firenze in visita a Lamporecchio venivano accolte affettuosamente, mentre la mia bisnonna Amelia, la moglie legittima, si chiudeva sdegnosamente in camera sua. Per me Albertina è stata una sorta di strana e simpatica zia fiorentina, anche lei una outsider: illegittima, intelligente, ironica, brutta e povera.

Mia nonna sembrava non essersi accorta del peso insostenibile che il padre le aveva affidato, affascinata come era da lui e dall'orgoglio di essergli stata necessaria. «Loro non lo capivano - mi diceva, riferendosi ad Albertina e Sofia - tanto che è venuto a morire a Lamporecchio, da me».

Durante la guerra, dopo la perdita della figlia Rita, morta a vent'anni, angosciata per il figlio, dato per disperso in Africa, Velia cadde in una grave crisi psichica e fu ricoverata per un anno in un istituto psichiatrico. Ho trovato un quaderno in cui, in quei momenti di delirio, scriveva a lapis con mano malferma parole che le rammentavano forse i garofani rossi del padre: Compagni, avanti! Il gran partito / noi siamo dei lavorator... 
Bibliografia

Aiazzi R.L., Papini Piquè S., Cecchi L. (a cura di), Inventario dell'Archivio del Comune di Lamporecchio, Comune di Lamporecchio, Lamporecchio 2014.

Andreucci F., Pescarolo A., La formazione delle regioni "rosse" in Italia: il caso della Toscana, in Gli spazi del potere. Aree, regioni, Stati: le coordinate territoriali della storia contemporanea, a cura di Id., La Casa Usher, Firenze 1989.

Archivio Domizio Torrigiani, inventario a cura di Eugenia Corbino, ISRT, Firenze 2012.

Bagnasco A., Tre Italie. La problematica territoriale dello sviluppo italiano, il Mulino, Bologna 1977.

Baronti N. (a cura di), Befanate e scherzi in poesia, Raccolta di poesie, prose poetiche e stornelli della tradizione orale di Vinci e del Montalbano, Polistampa, Firenze 2011.

Bartolini S., Una passione violenta. Storia dello squadrismo fascista a Pistoia (1919-1923), Comune di Pistoia, Pistoia 2011.

-, La mezzadria nel Novecento. Storia del movimento mezzadrile tra lavoro e organizzazione, Settegiorni, Pistoia 2015.

Becattini G., Scritti sulla Toscana, 4 voll., Le Monnier, Firenze 2007.

Becchetti M., Fuochi oltre il ponte. Rivolte e conflitti sociali a Parma (1868-1915), Derive Approdi, Roma 2013.

Bencistà A., Idalberto Targioni, «Toscana folk», III (4), 1999, pp. 32-34.

-, L'ambulante scuola. Breve storia dell'ottava rima in Toscana, Pupi e Fresedde, Regione Toscana, 2005, <http://www.pannostrale.it/improvvisatori.html>.

Bertolucci F., Antonio Gamberi, ateo convinto e fermo socialista. Appunti per una biografia, in A. Gamberi, Poesieper un "liberato mondo". Antologia, a cura di F. Bertolucci e D. Ronco, BFS, Pisa 2004, pp. 11-56.

Bianchi R., Il centro in periferia. Società e politica nella Valdelsa contemporanea (19001980), in P. Ginsborg, F. Ramella (a cura di), Un'Italia minore. Famiglia, istruzione e tradizioni civiche in Valdelsa, Giunti, Firenze 1999, pp. 32-108.

-, Bocci-Bocci. I tumulti annonari nella Toscana del 1919, Olschki, Firenze 2001.

-, Donne di Greve. Primo maggio 1917 nel Chianti. Donne in rivolta contro la guerra, Odradek, Roma 2004.

-, Pace, pane, terra. Il 1919 in Italia, Odradek, Roma 2006.

-, La guerra di Piero. Calamandrei tra Grande guerra e fascismo, «Antologia Vieusseux», 40, 2008, pp. 201-205. 
-, Il fronte interno alla prova. Le opposizioni alla guerra a Prato e in Toscana, in D. Menozzi, G. Procacci, S. Soldani (a cura di), Un paese in guerra. La mobilitazione civile (1914-1918), Unicopli, Milano 2010, pp. 105-132.

-, Grande guerra, Grande dopoguerra. Lotte politiche e conflitti sociali a Pistoia (19141921), in Pistoia nell'Italia unita. Identità cittadina e coscienza nazionale, Società pistoiese di Storia patria, Pistoia 2012, pp. 263-290.

-, La massoneria e le origini del fascismo in Toscana, «Contemporanea», XVI (4), 2013, pp. 499-522.

-, Massoneria e fascismo in Toscana, in F. Conti (a cura di), La massoneria italiana da Giolitti a Mussolini. Il gran maestro Domizio Torrigiani, Viella, Roma 2014, pp. 109-138.

- (a cura di), La Grande Guerra lontano dal fronte. Barberino Val d'Elsa e Tavarnelle Val di Pesa 1914-1918, Pacini, Pisa 2018.

-, Targioni, Idalberto, in Dizionario Biografico degli Italiani, Istituto dell'Enciclopedia italiana Treccani, Roma, in corso di pubblicazione.

Boldrini F., Minatori di Maremma. Vita operaia, battaglie sindacali e lotte politiche a Ribolla e nelle Colline metallifere (1860-1915), introduzione di I. Tognarini, Comune di Roccastrada, Roccastrada 2006.

Brotini M., Nuova linfa al manifesto di Marx. In ottava rima, «Left», 26 dicembre 2017, <https://goo.gl/qCtQ9Z>.

Calamandrei P., Zona di guerra. Lettere, scritti e discorsi (1915-1924), a cura di S. Calamandrei e A. Casellato, Laterza, Roma-Bari 2006.

Cammarano F., Dalla preghiera al tumulto: un'eccedenza alla ricerca della politica, in Id. (a cura di), Abbasso la guerra! Neutralisti in piazza alla vigilia della Prima guerra mondiale, Le Monnier, Firenze 2015, pp. 1-16.

Cantagalli R., Storia del fascismo fiorentino 1919/25, Vallecchi, Firenze 1972.

Cerasi L., Democrazia del lavoro, laicismo, patriottismo: appunti sulla formazione politica di Domizio Torrigiani, in F. Conti (a cura di), La massoneria italiana da Giolitti a Mussolini. Il gran maestro Domizio Torrigiani, Viella, Roma 2014.

Cioppi E., 18 maggio 1895: storia di un terremoto fiorentino, Osservatorio Ximeniano, Firenze 1995.

Conti F., Dalla sociabilità d'élite all' associazionismo di massa: società civile e riti della modernità a Pistoia nell'età liberale, in G. Petracchi (a cura di), Storia di Pistoia, vol. IV, Nell'età delle rivoluzioni, 1777-1940, Le Monnier, Firenze 2000.

-, Storia della massoneria italiana. Dal Risorgimento al fascismo, il Mulino, Bologna 2003.

Di Bello G., Senza nome né famiglia. I bambini abbandonati nell'Ottocento, Manzuoli, Firenze 1989.

-, L'identità inventata. Cognomi enomi dei bambini abbandonati a Firenze nell'800, Centro Editoriale Toscano, Firenze 1993.

Dogliani P., Il fascismo degli italiani. Una storia sociale, Utet, Torino 2014.

Fabbri F., Le origini della guerra civile. L'Italia dalla Grande Guerra al fascismo, 19181921, Utet, Torino 2009.

Fasano Guarini E., Petralia G., Pezzino P., Storia della Toscana, vol. 2: Dal Settecento a oggi, Laterza, Roma-Bari 2004.

Filipponi S., Mazzocchi E., Sandri L. (a cura di), Figli d'Italia (1861-1911). Gli innocenti e la nascita di un progetto nazionale per l'infanzia, Alinari 24 ore, Firenze 2011. 
Franceschini F., I contrasti in ottava ria e l'opera di Vasco Cai da Bientina, Pacini, Pisa 1983.

Francini M., Primo dopoguerra e origini del fascismo a Pistoia, Feltrinelli, Milano 1976.

Francini M., Balli G.P., Il "gran maestro" Domizio Torrigiani (1876-1932), CRT, Pistoia 2003.

Franzina E., Dall'Arcadia in America. Attività letteraria ed emigrazione transoceanica in Italia (1850-1940), Fondazione Giovanni Agnelli, Torino 1996.

Gagli G., La Baccheide (Poema eroicomico in ottava rima). La rivolta contro la guerra delle donne di Greve in Chianti 1 maggio 1917, a cura di C. Baldini, Comune di Greve in Chianti, Greve in Chianti 1998.

Gibelli A., L'officina della guerra. La Grande guerra e le trasformazioni del mondo mentale, Bollati Boringhieri, Torino 1991.

Gissi A., Le segrete manovre delle donne. Levatrici in Italia dall'Unità al fascismo, Biblink, Roma 2006.

Guarnieri P., Dall'accoglienza alla cura: la riforma sanitaria nel brefotrofio degl'Innocenti di Firenze 1890-1918, «Medicina \& storia», 7, 2004, pp. 57-100.

Guerrini L., Un poeta estemporaneo, Idalberto Targioni, nella storia del movimento contadino dell'empolese, «Movimento operaio», VII (3-4), 1955, pp. 511-530.

—, Il movimento operaio nell'Empolese 1861-1946, Editori Riuniti, Roma 1970, pp. 114-115.

I canti della tradizione popolare toscana. Bibliografia, Regione Toscana, Firenze 2017.

Isnenghi M., Convertirsi alla guerra. Liquidazioni, mobilitazioni e abiure nell'Italia tra il 1914 e il 1918, Donzelli, Roma 2015.

Isnenghi M., Rochat G., La Grande Guerra 1914-1918, La Nuova Italia, Firenze 2000.

Labanca N. (a cura di), Dizionario storico della Prima guerra mondiale, Laterza, RomaBari 2014.

Lancisi M., Berlusconi ordinò «Detoscanizzare l'Italia», ma la regione rossa ha resistito alla sfida, «Il Tirreno», 13 novembre 2011.

Lovito D., Empoli tra Grande Guerra e fascismo (1914-1921), Tesi di laurea magistrale, Università degli Studi di Firenze, rel. R. Bianchi, a.a. 2013/2014.

-, La Grande Guerra in una piccola città, «Quaderni d'Archivio», V (5), 2015, pp. 27-51.

Lyttelton A., La conquista del potere. Il fascismo dal 1919 al 1929, Laterza, Roma-Bari 1982.

Manfredi M., Il neutralismo anarchico, in F. Cammarano (a cura di), Abbasso la guerra! Neutralisti in piazza alla vigilia della Prima guerra mondiale, Le Monnier, Firenze 2015 , pp. 57-69.

Martini L., In memoria di Idalberto Targioni uomo prima tanto amato, poi tanto vituperato e, infine, troppo presto dimenticato, «Il Segno di Empoli», VI (21), 1993, pp. 8-9.

Montesi B., Un'«anarchica monarchica». Vita di Maria Rygier (1885-1953), Esi, Napoli 2013.

Morelli A., Targioni Idalberto, in F. Andreucci, T. Detti (a cura di), Il movimento operaio italiano. Dizionario biografico 1853-1943, Editori Riuniti, Roma 1978, vol. V, pp. 6-13.

Morelli A., Tomassini L., Socialismo e classe operaia a Pistoia durante la prima guerra mondiale, Feltrinelli, Milano 1976.

Mori G. (a cura di), Storia d'Italia. Le regioni dall'Unità a oggi. La Toscana, Einaudi, Torino 1986. 
Moroni S., Massoneria e vita politica a Pistoia e nella Val di Nievole tra guerra e fascismo, in F. Conti (a cura di), Massoneria e società civile. Pistoia e la Val di Nievole dall'Unità al secondo dopoguerra, FrancoAngeli, Milano 2003, pp. 97-129.

Neri Serneri S., Il localismo come vincolo e come risorsa. Note sul caso toscano, in I. Milanese (a cura di), Le Camere del lavoro italiane. Esperienze storiche a confronto, Longo, Ravenna 2001, pp. 191-207.

Nesti A., Provincialia. Scavi sull'identità degli italiani, Polistampa, Firenze 1999.

Padulo G., Dall' interventismo al fascismo, in G.M. Cazzaniga (a cura di), Storia d'Italia, Annali, 21, La massoneria, Einaudi, Torino 2006, pp. 657-700.

Palla M., Firenze nel regime fascista (1929-1934), Olschki, Firenze 1978.

Perugi G., Alle urne! Le elezioni politiche del 1913 nelle cronache dei giornali locali, in E. Galli, G.C. Romby (a cura di), Voci di carta. Informazione e formazione nei giornali locali del primo Novecento, Settegiorni, Pistoia 2012.

Petracchi G., La genesi del fascismo a Pistoia, in 28 ottobre e dintorni. Le basi sociali e politiche del fascismo in Toscana, Polistampa, Firenze 1994, pp. 97-121.

—, Storia di Pistoia, vol. IV: Nell'età delle rivoluzioni 1777-1940, a cura di Id., Le Monnier, Firenze 2000.

Pezzino P., Empoli antifascista. I fatti del $1^{\circ}$ marzo 1921, la clandestinità e la Resisten$z a$, Pacini, Firenze 2007.

Poesio C., Firenze, in F. Cammarano (a cura di), Abbasso la guerra! Neutralisti in piazza alla vigilia della Prima guerra mondiale, Le Monnier, Firenze 2015, pp. 447-458.

Poli C., Lamporecchio nel primo Novecento. Quotidianità e istituzioni, Pistoia, CRT, 2004.

- La vicenda del socialista Targioni e la nascita del fascismo a Lamporecchio, «Quaderni di Farestoria», XIII (2-3), 2011, pp. 133-141.

Poli S., L'uomo che voleva detoscanizzare l'Italia, «la Repubblica», 23 gennaio 2010.

Priore D., L'ottava rima, Comune di Terranova Bracciolini, Terranova Bracciolini 2002.

Procacci Giu., La lotta di classe in Italia agli inizi del secolo XX (ed. orig. 1970), Editori Riuniti, Roma 1992.

Putnam R.D., La tradizione civica nelle regioni italiane, Mondadori, Milano 1993.

Ragionieri E., Il partito fascista (appunti su una ricerca), in La Toscana nel regime fascista (1922-1939), vol. 1, Olschki, Firenze 1971, pp. 72-73.

Ricci A., Detto e taciuto. Le ottave del consenso contadino al regime fascista, «La Ricerca Folklorica», 11, 1985, pp. 121-124.

Risaliti R., Il movimento socialista a Pistoia durante la Prima guerra mondiale, Comune di Pistoia, Pistoia 1970.

—, La lotta sindacale e politica a Pistoia dal dicembre 1918 al luglio 1920, Comune di Pistoia, Pistoia 1970.

-, Nascita e affermazione del fascismo a Pistoia, «Farestoria», III (1), 1983.

Ronconi E., Antifascismo borghese 1922-1930, «Italia contemporanea», 1980, 140, p. 20.

Sabbatucci G., Vidotto V. (a cura di), Storia d'Italia, vol. 4: Guerre e fascismo 1914-1943, Laterza, Roma-Bari 1997.

Savelli L., L'industria di montagna. Uomini e donne al lavoro negli stabilimenti della Società Metallurgica Italiana, Olschki, Firenze 2004.

Scirocco G., Il neutralismo socialista, in F. Cammarano (a cura di), Abbasso la guerra! Neutralisti in piazza alla vigilia della Prima guerra mondiale, Le Monnier, Firenze 2015, pp. 41-55. 
Snowden F., The Fascist Revolution in Tuscany 1919-1922, Cambridge University Press, Cambridge 1990.

Soldani S., La Grande Guerra lontano dal fronte, in G. Mori (a cura di), Storia d'Italia. Le regioni dall'Unità a oggi. La Toscana, Einaudi, Torino 1986, pp. 426-452.

Targioni I., Il terribile terremoto a Firenze e nei paesi circonvicini. Narrazione dell'estemporaneo Idalberto Targioni, Tip. Salani, Firenze 1895.

- Contrasto tra un prete democratico-cristiano e un contadino ascritto alla Lega di resistenza, Sez. socialista di Empoli, Empoli 1902.

—, Terza risposta del Targioni al Manuelli, Tip. E. Severi, Firenze 1902.

- Caprera: La casa e la tomba di Giuseppe Garibaldi: terzine, Tip. Niccolai, Pistoia 1904.

-, L'avvenire: canto epico del poeta estemporaneo Idalberto Targioni, Tip. Traversari, Empoli 1905.

- Ilprete attraverso le religioni e la storia: poemetto eroicomico in ottava rima del poeta estemporaneo Idalberto Targioni, Tip. U. Polli, Firenze 1906.

-, Martiri del lavoro: sestine, Ciattini, Pistoia 1906.

—, Gli scandali clericali, Tip. Cooperativa, Pescia 1907.

-, Agli emigrati italiani, Tip. L. Migliorini, Zurigo 1908.

—, L'incendio della miniera: Bozzetto sociale in versi, Tip. I. Cecchi e C., Prato 1908.

-, Dalla nascita alla morte della terra: Conferenza in versi, tenuta la prima Volta all' $U$ niversità popolare di Zurigo il 10 Maggio 1909, Tip. Fratelli Ciattini, Pistoia 1909.

-, Il terremoto calabro-siculo: Bozzetto sociale in versi martelliani, Tip. Fratelli Ciattini, Pistoia 1910.

-, Civiltà borghese o tirannide capitalistica? Terzine, Tip. U. Polli, Firenze 1911.

-, Per l'educazione della donna secondo la morale cristiana e socialista: contradittorio in ottava rima tenuto dai due poeti improvvisatori Fortunato Chiti e Idalberto Targioni la sera del 19 marzo 1911 nella Società di M. Soccorso di Varlungo (Firenze), resoconto stenografico, Tip. Polli, Firenze 1911.

-, Risposta per le rime alla canzone d'oltremare di Gabriele d'Annunzio, Tip. U. Polli, Firenze 1911.

-, Canzoniere di poesie sociali, Tip. U. Polli, Firenze 1912.

-, Ettore Giovannitti e l'emigrazione transoceanica, Tip. Ugo Polli, Firenze 1912.

-, I miei ricordi d'infanzia, ovvero I "proverbi" di mamma Giuditta, Polli-Targioni, Firenze 1912.

—, Piccoli parassiti: Opuscolo di propaganda, Tip. U. Polli, Firenze 1912.

—, Idalberto Targioni, «Il Popolano», 26 maggio 1915.

—, Perché me ne vado, «Il Nuovo Giornale», 17 maggio 1916.

—, Dimissioni clamorose, «Il Popolo pistoiese», 20 maggio 1916.

-, Vent'anni di propaganda e cinque anni d'Amministrazione Socialista nel Comune di Lamporecchio, Tip. Guido Grazzini, Pistoia 1920.

—, La parola all'imputato, «L'Alleanza», 4 marzo 1921.

-, I doveri del contadino, «La Riscossa», 3 giugno 1922.

-, Calendario agricolo fascista, anno 1930, 8. E. F., vade mecum del contadino Toscano, Tip. Fascista, Firenze 1930.

Terzaghi M., Fascismo e Massoneria, Editrice Storica, Milano 1950.

Tomassini L., Carlo Corsi, in F. Andreucci, T. Detti (a cura di), Il movimento operaio italiano. Dizionario biografico 1853-1943, Editori Riuniti, Roma 1978, vol. II, pp. 99-100. 
-, Terzaghi Michele, in F. Andreucci, T. Detti (a cura di), Il movimento operaio italiano. Dizionario biografico 1853-1943, Editori Riuniti, Roma 1978, vol. V, pp. 51-55.

-, Associazionismo operaio a Firenze fra '800 e '900. La Società di mutuo soccorso di Rifredi (1883-1922), Olschki, Firenze 1984.

Turi G., Intellettuali e propaganda nel movimento socialista, in Fare gli italiani. Scuola e cultura nell'Italia contemporanea, a cura di Id. e Simonetta Soldani, vol. 1: La nascita dello Stato nazionale, il Mulino, Bologna 1993, pp. 459-501.

-, Schiavi in un mondo libero. Storia dell'emancipazione dall'età moderna a oggi, Laterza, Roma-Bari 2012. 


\section{Indice dei nomi}

Abamelech Simone, 59

Acciarito Pietro, 53

Aiazzi Pasquale, 113

Aiazzi Rita Laura, 15, 153

Alati Demetrio, 123

Alberti Carlo, 66

Alberti Nerina, 66

Alfieri famiglia, 118

Alfieri Vittorio 144, 146, 151

Alighieri Dante, 140-141, 145

Allori Romualdo, 117

Ambrogini Poliziano Angelo, 141

Ancillotti Leopoldo, 90

Andreucci Franco, Ix, 153, 155, 157-158

Angiolini Alfredo, 127, 135

Antinori Orazio, 143

Ardigò Roberto, 47

Argante, 90

Ariosto Ludovico, 15, 142

Armani Evaristo, 113

Armelani Francesco, 136

Bacarelli famiglia, 12

Bacarelli Rizieri, 118, 122

Bacarelli Sofia, 12-13, 19, 39, 57, 60, 65-

$66,118,122$

Bacarelli Targioni Idalberta, 12-13, 19, $33,39,57,60,76,81,86-92,152$

Bacci Giovanni, 123

Baccini-Ferri Ida, 142, 151
Baccioni Enrica, 66

Badaloni Nicola, 28, 125

Badoglio Pietro, 28

Bagnasco Arnaldo, viII, 153

Bagnoli Gaetano, 112

Bagnoli Primo, 102

Balbo Cesare, 144, 151

Baldini Carlo, 15, 155

Balli Gian Paolo, x, 11, 155

Balsimelli Cesare, 29

Bandiera Attilio, 55

Bandiera Emilio, 55

Bandinelli, 91

Bandini Vittorio, 99

Baretti Giuseppe, 144, 151

Baronti Nicola, x, 153

Bartolini Gastone, 113

Bartolini Stefano, x, 24, 153

Bartolozzi Ermindo, 99

Bassi Ugo, 55

Basso Maurizio, 136

Battisti Cesare ,56,70

Bazzi Tullio, 116

Becattini Giacomo, IX, 153

Becchetti Margherita, 23, 153

Bellini Vittorio, 64

Bellotti Pietro, IX

Bencistà Alessandro, 5, 153

Benedetti Silvio, 116, 132

Benelli Francesco, 69 
Benesperi Antonio, 126

Benozzi Angiolo, 114

Benuzzi F., 123

Berenini, 28

Berni Francesco, 5, 15, 24, 29, 119, 129

Berta Giovanni, 65

Bertolucci Franco, x, 153

Bertuccelli famiglia, 118

Bettarini Benvenuto, 91

Bianchi Enrico ,141

Bianchi Ferruccio, 64

Bianchi Michele, 114

Bianchi Pietro, 131-132, 135

Bianchi Roberto, IX-X, 9, 11, 15-17, 20, 24-25, 28-29, 153, 155

Biondi Pietro, 25

Bisso Giovanbattista, 137

Bissolati Leonida, 28

Boccaccini Telemaco, 70

Bocci Paolo Anastasio, 138, 142

Boldrini Fabrizio, 13, 154

Bondi Giuseppe, 64

Bonfatti, 24, 32, 66

Bonsanti Marta, XII

Bordoni Iacopo, 143

Borghi Pier Paolo, 121

Borgioni Tito, 118

Borri, 114

Botta Carlo, 145, 151

Bracali Donatello, ,71, 106, 137

Bregonzio Giuseppe, 112

Brotini Maurizio, x, 154

Bruni Alessandro, 89-90, 121

Bruni Bruno, 121

Bruno Giordano, 121, 137, 151

Burci Enrico, 56, 61-62, 74-75

Burdati, 119

Burgassi, 74, 85-86, 88

Cabrini Angiolo, 28, 126

Cadorna Luigi, 21

Cairoli Angelo, 50

Calamandrei Piero, 20, 153-154

Calamandrei Silvia, 20, 154

Calani Amelia, 137

Calzolai Nello, 97

Cambellotti, 114

Cammarano Fulvio, 17, 23, 154-156
Cammilli Edoardo, 90, 95-97

Campanella Tommaso, 141, 151

Canonico Schuller Ludovico, 143

Cantagalli Roberto, 27-28, 154

Cantalupo Roberto, 92

Capecchi Domenico, 4, 6-7, 10

Carbonai Guido, 93, 107-108, 113

Carbonici Bernardino, 13

Carducci Giosuè, 46, 58, 122, 141, 144, 151

Carnevale Salvatore, IX, 69

Casellato Alessandro, 20, 154

Casini Tommaso, 141

Castagno Gino , IX

Casti Gianbattista, 142

Catolfi Cesare, 96

Catolfi Olinto, 96-97, 99

Cavallotti Felice, 138-139, 143-144, 151

Cavour Camillo Benso, 88, 138, 151

Cazzaniga Gian Mario, 29, 156

Cecchi Lucia, 13, 15, 50, 136, 144, 153, 157

Cei Eliseo, 25

Cenci Beatrice, 137

Cerasi Laura, 11, 154

Cesare Giulio, 51

Cesari Emidio, 126

Cesarotti Melchior, 139

Cetraro Monica, XII, 4

Chiappelli Alessandro, 47

Chiappini Dina ,118

Chiappini famiglia, 50

Chiappini Idalberto, 49, 88-89, 117

Chiappini Lida, 29, 41-42, 49, 70, 93, $117,121,127$

Chiappini Rita, 9, 117

Chiappini Simonetta, xi-XII, 2, 9, 29, 4950,70-71,73-74, 79, 81, 86, 88-89, 93, 109, 117-118, 120-121, 127, 132, 149

Chiappini Torello, 9, 42, 71, 73-74, 79, 81-82, 85-86, 88-90, 109, 132

Chiavacci Egidio, 22

Chini Ferdinando, 65

Chiti Filomena, 119

Chiti Fortunato, 13, 45, 48, 53, 58, 65, $68,79,119-120,122,157$

Ciampi Giovanni, 111

Cianchi Mario, 122 
Ciano Costanzo, 102, 111, 113

Ciappi Gaetano, 58

Cicerone Marco Tullio, 141

Cioletti Luigi, 33, 100

Cioli famiglia, 105

Cioppi Elisabetta, 9, 154

Ciotti Pompeo ,123, 125

Cipulat Amulio, 22

Colajanni Napoleone, 23, 55

Colonna Vittoria, 51

Comba Eugenio, 139

Comolli Antonio, 62

Compagni Giovanni, 20, 52, 55, 90, 102, $106,112-114,135,137,151-152$

Conti Fulvio, x, 11, 29, 154, 156

Corbino Eugenia, 11, 153

Cornelio Tacito, 141

Corsi Carlo, 22, 136, 157

Costa Andrea, 128

Costa Paolo, 140-141, 145

Crevier Jean Baptiste Louis, 138, 151

Cristiani Ferruccio, 113

Daddi, 74, 84-85, 88

Dalla Volta Riccardo, 48

Dandolo Emilio, 143

D’Annunzio Gabriele, 48, 68, 141

Da Pistoia F. Romolo, 143

Da Porretta F. Felice, 126

Darwin Charles, 140

Dati Irene, XII

De Amicis Edmondo, 126, 136, 151

De' Colli Niccolò, 63

De Fereal Victor, 143

De Lamartine Alfonso, 144

Delcroix Carlo, 105, 116

Del Grosso Angelo, 101

Della Pura Alfredo, 139

Della Sete Alceste, 125

De Maria Federico, 126

De' Medici Lorenzo, 141

Demidoff Maria, 59

De Montépin Xavier, 137

De’ Rossi Giulio, 125

Detti Tommaso, IX, 155, 157-158

Di Bello Giulia, 154, xi

Di Marco Datini Francesco, 126

Dinale Ottavio ,124
Dioniso Longino, 144

Di Pol Bruno, IX

Dogliani Patrizia, x, 154

Domela Niuwenhuis F., 123

Donati Orlando, 99, 117

Dumas Alexandre, 136

Dunn Andrea, 143

Eschilo, 144, 151

Fabbri Fabio, 25, 154

Fandi, 99

Fanti Alberto, 114

Fanti Alfredo, 57

Fanti Ezio, 25

Fanti Telemaco, 52, 71

Fantoni Giovanni, 143

Farinacci Roberto, 102

Farina Salvatore, 142

Fasano Guarini Elena, 9, 154

Fedi Amerigo, 97

Fedi Otello, 58

Ferri Enrico, 47, 70, 124-125, 129, 142, 151

Ferrucci Francesco, 51

Filipponi Stefano, xi, 154

Fiorineschi Michele, 71

Florio Federigo Guglielmo, 103, 105, 111

Forlani Ugo, 22

Fornaciari Luigi, 139

Forteguerri Niccolò (Carteromaco), 151

Foscolo Ugo, 145, 151

Franceschi Pignocchi Teodolinda, 142, 151

Francini Marco, x, 11, 155

Franzina Emilio, $\mathrm{X}, 155$

Frilli Alfredo, 22

Frizzolani Otello, 99

Frosini Attilio, 69

Gaggioli Enrico, 116

Gagli Galileo, 15, 155

Galeotti Ezio, 51, 104, 109, 119, 126

Galeotti Giulia, 126

Galeotti Luigi, 119

Galilei Galileo, 141

Galli Emanuela, 13, 156

Galluzzi Riguccio, 145, 151 
Galvani Francesco, 140

Gambacciani Italo, 132

Gamba Giuseppe, 136

Gamberi Antonio, x, 153

Garibaldi Giuseppe, 12-13, 51, 58, 71, $80,122,157$

Gasperini F., 12, 52, 54, 87, 118, 143

Gasperini Renzo, 12, 52, 91-92, 152

Gasperini Tranquillo, 12, 52, 54, 118, 143

Gatti Girolamo, 125

Gavazzi Barbara, 118

Gavazzi Bartolomeo, 58

Gavazzi Giovanni, 2-3, 58, 118

Gavazzi Mazzina, 2

Gay Teofilo, 140

Giacchi Oscar, 137

Giacomelli Persindo, 102

Giannelli Andrea, 138

Giannetti G., 143

Giannini Ferruccio, 100

Giannini Pietro, 117

Giannoni Ugo, 118, 130

Gibelli Antonio, viII, 155

Ginanni Luigi, 115

Ginsborg Paul, IX, 153

Gioja Melchiorre, 144, 151

Giolitti Giovanni, x, 11, 63, 130, 154

Giovannitti Ettore, 13, 46, 135, 157

Giraldi Alipio, 99

Giraldi Federico, 119

Giraldi Giuditta, viI, 4, 7, 44, 46, 48, 135, $150,152,157$

Gissi Alessandra, 1, 155

Giuseppe Monti, 51, 69

Giusti Giuseppe, 116, 125, 135, 139, 151 , VII

Gneo Marcio Coriolano, 51

Gnoli Teresa, 143, 151

Gonnelli Elena, XII

Goretti Leo, 16

Gori Anton Francesco, 144

Gori Pietro, 123-124, 142

Gragnani Irene, 1

Grossi Tommaso, 137, 151

Grou-Radenez Justine, XII

Guarnieri Patrizia, xi, 155

Guatelli Fulvio ,XII
Guazzini Vincenzo, 69, 126

Guerrazzi Francesco Domenico, 13, 124, 136-137, 144, 151

Guerrazzi Vincenzo, 13

Guerrini Libertario, IX, 8, 10, 17, 33, 121,155

Guidi Giuseppe, 69

Guidi Tommasina, 142, 151

Hegel Carlo, 140

Hugo Victor, 139-140

Innocenti Elisa, 62

Innocenti Ferdinando, 55

Innocenti Gino, 105

Innocenti Lindoro, 119

Isnenghi Mario, 18, 23, 155

Jaurès Jean, 15

Jozzelli Jacopo, 138

Labanca Nicola, 21, 155

Labriola Arturo, 138, 151

Lancetta Pietro, 142

Lancisi Mario, VIII, 155

Lanconi Ada, 90

Lanconi Amerigo, 90

Lapucci Pietro, 125

Larini Guido, 45

Larini Pietro, 73

Lassi Guido, 64

Lauda, 87

Lazzari Costantino, 122

Lazzerini Ettore, 47

Lenzi Ilio, 109, 111

Leone Enrico, 48, 128

Leopardi Giacomo, 145, 151

Leporatti Santo, 115

Lisi Simone, 16

Lombroso Cesare, 145, 151

Longobardi Ernesto Cesare, 125

Lovito Daniele, xi-XII, 17, 155

Lucchesi Antonio, 27

Luchetti Lorenzo, 143

Lucini Gian Pietro, 140

Luisa Martini, 155

Lupi Dario, 113

Lyttelton Adrian, viII, 34, 155 
Maccheroni Fausto, 62

Macé Giovanni, 145

Machiavelli Niccolò, 138, 151

Maffi Fabrizio, 47

Maggianti, 113

Malatesta Errico, 125

Malespini Dino, 137

Malon Benoît, 121

Mancini Ilio, 78, 89-91, 117, 136

Mancini Lorenzo, 136

Manfredi Marco, 23, 155

Mantegazza Paolo, 137-139, 141, 144, 151

Manuelli Luigi, 13, 43-44, 48, 157

Marchi Giovanni, 92

Marelli famiglia, 100

Mariani Franco, IX

Mariani Gustavo, 113

Mari Morando, 110

Marinetti Filippo Tommaso, 140

Marini Angelo, 142

Mario White Jessie, 130, 136

Marradi Guido, 99

Martelli Attilio, 97

Martelli Carlo, 131

Martini Ferdinando, 125, 141

Martini Giovanni, 126

Martini Luisa, IX, xi, 4, 49, 67, 155

Martini maestro, 108

Martinuzzi Giuseppina, 126

Marx Karl, 11, 151

Masi Attilio, 116

Massai Saul, 116

Matcovich Enrico, 142

Matteotti Giacomo, 29, 92

Matteucci famiglia, 119

Mazzarella Bonaventura, 137

Mazzei Aldo, 114

Mazzini Giuseppe, 69, 123, 125, 130, $136,138,151$

Mazzocchi Eleonora, xi, 154

Mazzoni Giuseppe, 103, 144

Mazzoni Matteo, XII

Mazzurri, 98

Menozzi Daniele, 17, 154

Menzini Benedetto, 139

Meoni Dario, 25, 124

Meozzi Augusto, 118

Meozzi Cesare ,25
Meozzi Eugenia, 117

Meozzi Luigi, 118

Merlini Alfonso, 109, 117

Merlino Cinzia, XII

Merrick Laura T., 67, 81, 117

Migliorati Carlo, 70, 120-121

Milanese Isabella, 12, 138, 156

Milani Luciano, 121

Milhaud Edgard, 124

Minghetti Al., 93

Minghetti Antenore, 93, 99

Minghetti Carlo, 132

Minghetti Francesco, 70, 128

Miniati Augusto, 105

Modigliani Giuseppe Emanuele, 70

Molinari Simona, IX

Montanelli Giuseppe, 144

Montemartini Luigi, 122

Montesi Barbara, 23, 155

Montgomery Florence, 141, 151

Monticelli C., 125

Monti Vincenzo, 139, 151

Monzani Fazio ,118

Morand Charles, 144

Morelli Aldo, IX, 22, 117-118, 155

Morelli Berto, 118

Morelli Iola, 118

Morelli Luigi, 117

Morgari Oddino, 124

Moriani Giorgio, 102

Mori Giorgio, VIII, 9, 155, 157

Moroni Sheyla, x, 156

Morosi Azelio, 97, 100, 118

Morselli Enrico, 140, 151

Mottura Paolo, 140

Mussolini Benito, VIII, x, 11, 21-22, 24, $28,30,34,48,59-61,67,154$

Naldi Leopoldo, 50

Nasti Agostino, 66

Nazzurri Guido, 99

Neera (Anna Zuccari), 141, 151

Neri Alfredo, 99

Neri Giuseppe, 99

Neri Serneri Simone, XII, 12, 156

Nerozzi Gina, 64

Nesi, 112

Nesti Arnaldo, x, 156 
Niccolai Amelia, 93

Niccolai Felice, 93

Niccoli Cesare, XII, 4, 37, 136

Niccolini Giovan Battista, 136, 143, 146, 151

Odett Santini Umberto, 60

Omero, 118, 126, 136, 139

Onorato Luigi, 141

Orsi Alberto, 144

Orvieto Angelo, 47

Pacchiani Verdiani Bettina, 117

Pacini Gino, 113, 132

Pacini Guido, 51

Pacini Monica, XII

Pacini Pacino, 107

Pacini Silvio, 140

Padulo Gerardo, 29, 156

Paganelli Santi, 99

Paganini Virginia, 122-124, 144, 151

Pagano Francesco Mario, 142, 151

Pagliai Teresa, 106

Palandri Annibale, 44

Palla Marco, 20, 26, 29, 156

Pancani famiglia, 106, 118-119

Pancani Francesco, 118

Pancani Luigi, 106

Papini Giovanni, 15, 47, 63, 153

Papini Piquè Selena, 15, 153

Pasanisi Francesco Maria, 142

Pascoli Giovanni, 50

Pasquali Alfredo, 128, 143

Pasquinucci Giuseppe, 116

Passerini Giuseppe Lando, 123, 141

Pastorello Giovanni, 126

Paterniuti Salvatore, 64

Pelegatti Mario, 114

Pellegrini Colombo, 124

Pellizza da Volpedo Giuseppe, VII

Pera Francesco, 142

Perrone Compagni Dino, 20, 90, 102, $106,112-114,135$

Perugi Giampaolo, 13, 156

Pescarolo Alessandra, IX, 153

Pescetti Giuseppe, 9

Petracchi Giorgio, x, 12, 22, 26, 28, 60, 154, 156
Petracchi Giuseppe, 60

Petragnani Umberto, 25

Petralia Giuseppe, 9, 154

Petrarca Francesco, 144, 151

Petrocchi P., 136

Pezzino Paolo, 9, 26, 154, 156

Philipson Dino, 28, 96-97, 131, 135

Picchi Scipione, 29

Pieri Goffredo, 66

Pini Oliviero, 117

Pisaneschi Pietro, 45

Pistolesi Luigi, 109

Pittoni Valentino, 130

Podrecca Guido, 47, 142

Poesio Camilla, 17, 156

Poggi Adolfo, 62, 137

Poggiali Amelia, 64

Poggi Rinaldo, 137

Poli Claudia, IX, 156

Poli Simona, VIII, 156

Pontanari Umberto, 102

Pontecchi Luigi, 106

Ponzia Alessandro, 62

Prati Giovanni, 141, 151

Procacci Giovanna, VIII, 10, 17, 154, 156

Procacci Giuliano, 10, 156

Proudhon Pierre-Joseph, 139, 151

Puccini Roberto, 139

Puliti, 91

Putnam Robert David, vIII, 156

Ragionieri Ernesto, 22, 156

Rai Domenico, 1

Rai Luisa, 1

Ramella Francesco, IX, 153

Randazzi Abramo, 89

Rapisardi Mario, 58, 126

Raspanti Enrico, 66

Razza Luigi, 84, 88, 91

Renard Giorgio, 125

Ricci Antonello, 26, 129, 139, 156

Ricciardi Lucia, XII, 115

Ricciardi Pollini Ricciardo, 115

Riccio, 109

Ricotti Ercole, 139

Rigutini Giuseppe, 140

Risaliti Renato, x, 156

Riva Giuseppe , 142 
Rochat Giorgio, 18, 155

Roghi Vanessa, XII

Romagnini Angiolo, 6-7

Romagnoli Carlo, 102, 107, 111-112, 114115,126

Romby Giuseppina C., 13, 156

Ronco Daniele, x, 153

Ronconi Enzo, 26, 156

Rosadi Giovanni, 47

Rospigliosi Giulio, 95-96

Rozzi, 104

Ruskin Giovanni, 63

Rygier Maria, 23, 155

Sabbatucci Giovanni, viII, 156

Saccenti Giovan Santi, 143

Sacconi Anna, 7

Sacconi Giuseppe, 7

Salgari Emilio, 136

Salvemini Gaetano, 46-47

Sandri Lucia, xi, 154

Santirocchi Giannina, 117

Sarti G., 48

Sassoon Donald, 10

Savelli Laura, 12, 156

Scalzotto Angelo, 123

Scappucci Luigi, 142

Scarfoglio Edoardo, 47

Schobert Hedwig, 144

Scirocco Giovanni, 22, 156

Scorza Carlo, 111, 113

Sella Emanuele, 145

Serao Matilde, 141, 151

Servaddio Giulia, 140

Shakespeare William, 141, 151

Signorini Giuseppe, 142

Silvestri Pasquale, 118

Snowden Frank, x, 27-28, 157

Soave Francesco, 139

Soldani Simonetta, X, XII, 17, 154 , 157-158

Sparvieri Fernando, 55

Spinelli, 109

Spinoza Baruch, 63

Stanghellini Arturo, 47

Stecchetti Lorenzo, 137

Stianti Giustina, 119

Strada Carlo, IX
Strozzi Filippo, 146

Sue Eugène, 136, 151

Tacchi Francesca, XII

Tafini, 119

Talini Oreste, 78, 93, 95-97, 117

Talini Talino, 93

Tamburini Tullio, 114

Tarabusi Gianni, 93

Tarelli Paola, IX

Targioni Dusola, 91

Targioni Rita, 9, 38, 61, 65, 67, 81, 83, 89, $117,120,141,152$

Targioni Velia, 9, 12, 29, 31-33, 38, 42, $49,67,70-90,118,120,149,151-152$

Targioni Vincenzo, 96

Tasso Torquato, 51

Terzaghi Michele, 22, 157-158

Tesi Giuseppe, 17, 123, 155

Tieraffi, 89

Tofanelli Felice, 60

Tognarini Ivan, 13, 154

Tognetti, 51, 69

Tolstoj Lev, 137, 139

Tomassini Luigi, IX, 22, 155, 157

Tommaso Alberto, 62

Torricelli Cesare, 72

Torrigiani Antonio, 52

Torrigiani Domizio, VIII, xi, 9-12, 19-20, $29,32,100,117-118,153-155$

Torrigiani Elide, 9, 48-49

Torrigiani Giulio, 25

Torrigiani Virginia, 117-118

Tosi Igino, 6-7, 104

Toti Enrico, 57-60, 118

Toti Omero, 118

Tresanini Carlo, 53

Trinci, 83

Turati Filippo, 33, 123, 151

Turi Gabriele, $\mathbf{x}, 7,158$

Ubaldi Alessandro, 119

Ubaldi Lidia, 119

Umberto I di Savoia, 53, 140

Uss Giovanni, 58

Valeriani G., 107

Vannacci Afrisio, 9, 48-49, 71, 90 
Vannacci Leopoldo, 38, 50, 90, 124

Vannacci Targioni Amelia, 9, 12, 38, 7173, 75, 77, 79-81, 85, 89-90, 93, 98, $118-120,140,152$

Vannacci Vannina, 93

Vannini Vannino, 105, 143

Vannucci, 86

Vasari Giorgio, 141, 151

Venette Nicola, 145

Ventura Camillo, 124

Venturini Alfredo, 117

Venzi Adolfo, 102, 106, 110

Venzick Teresa, 146

Verdi Giuseppe, 53, 68, 136, 151

Verga Giovanni, 141

Verne Jules, 143

Verri Pietro, 146, 151

Vertua Gentile Anna, 141, 151

Vescovi Alfredo, 17, 25-26, 134

Vidotto Vittorio, VIII, 156
Vigliani Giacomo, 7,12

Villani Filippo, 137

Villani Matteo, 137

Villari Pasquale, 47

Virgilii Filippo, 144

Virgilio, 145

Vitelli Gerolamo, 47

Volpi Alberto, 98

Wilson Thomas Woodrow, 47

Zagaglia (L. De Fazio), 124

Zagra Giuliana, 43

Zanotti Francesco Maria, 142

Zatta Antonio, 138

Zola Émile, 63, 68, 136, 138, 145, 151

Zolli Euclide, 25

Zucchi Giovanna, 118

Zucchi Giuseppe, 118

Zuccoli Luciano, 141 


\section{Biblioteca di Storia \\ Titoli pubblicati}

Azzari M., Rombai L. (a cura di), Amerigo Vespucci e i mercanti viaggiatori fiorentini del Cinquecento

Bertini F., La democrazia europea e il laboratorio risorgimentale italiano (1848-1860)

Bianchi R., Una storia, un archivio. Idalberto Targioni nell'Italia tra Ottocento e Novecento

Bicchierai M., Una comunità rurale toscana di antico regime. Raggiolo in Casentino

Bourin M., Cherubini G., Pinto G. (a cura di), Rivolte urbane e rivolte contadine nell'Europa del Trecento. Un confronto

Campos Boralevi L. (a cura di), Challenging Centralism: Decentramento e autonomie nel pensiero politico europeo

Cerioni C., di Carpegna Falconieri T. (a cura di), I conventi degli ordini mendicanti nel Montefeltro medievale. Archeologia, tecniche di costruzione e decorazione plastica

Corsi D., Diaboliche maledette e disperate. Le donne nei processi per stregoneria (secoli $X I V-X V I)$

Corsi D., Duni M. (a cura di), «Non lasciar vivere la malefica $\gg$. Le streghe nei trattati e nei processi (secoli $X I V-X V I I)$

Doni Garfagnini M., L'idea di Europa nelle 'Vite' di Richelieu. Biografia e Storia nel Seicento

Doni Garfagnini M., Strumenti e strategie della comunicazione scritta in Europa fra Medioevo ed Età Moderna

Fiorentino A., Il commercio delle pelli lavorate nel Basso Medioevo. Risultati dall'Archivio Datini di Prato

Giagnacovo M., Appunti di metrologia mercantile genovese: un contributo della documentazione aziendale Datini

Guarnieri P., Senza cattedra. L'Istituto di Psicologia dell'Università di Firenze tra idealismo e fascismo

Lorini A., An intimate and contested relation. The United States and Cuba in the latenineteenth and early twentyeth

Melani I., «Di qua» $e$ «di là da’ monti». Sguardi italiani sulla Francia e sui francesi tra XV e XVI secolo

Michelet J., Il Rinascimento, a cura di Leandro Perini

Morozzi U., Storia dei conventi cappuccini toscani dalla fondazione al 1704. La storia dell'Ordine da un manoscritto inedito di Filippo Bernardi da Firenze

dell'Ordine da un manoscritto inedito di Filippo Bernardi da Firenze

Nigro G., Francesco di Marco Datini. L'uomo il mercante

Nigro G., Francesco di Marco Datini. The Man the Merchant

Orlandi A., «Ora diremo di Napoli». I traffici dell'area campana nei manuali di commercio

Passetti C., Tufano L. (a cura di), Femminile e maschile nel Settecento

Pinelli P., Tra argento, grano e panni. Piero Pantella, un operatore italiano nella Ragusa del primo Quattrocento 
Pitti B., Ricordi, a cura di Veronica Vestri

Poliziano A., "Coniurationis commentarium". Commentario della congiura dei Pazzi, a cura di Leandro Perini

Rucellai B., "De Bello Italico". La guerra d'Italia, a cura di Donatella Coppini

Soldani S., Enzo Collotti e l'Europa del Novecento

Vespucci A., Cronache epistolari. Lettere 1476-1508, a cura di Leandro Perini

Zarri G., Baranda Leturio N. (a cura di), Memoria e comunità femminili. Spagna e Italia, secc. XV-XVII / Memoria y comunidadesfemeninas. España e Italia, siglos XV-XVII

Zorzi A., La trasformazione di un quadro politico. Ricerche su politica e giustizia a Firenze dal comune allo Stato territoriale

Zorzi A. (a cura di), La civiltà comunale italiana nella storiografia internazionale. Atti del I convegno internazionale di studi (Pistoia, 9-10 aprile 2005) 

